\title{
Pharmacotherapy for trichotillomania (Review)
}

Hoffman J, Williams T, Rothbart R, Ipser JC, Fineberg N, Chamberlain SR, Stein DJ

Hoffman J, Williams T, Rothbart R, Ipser JC, Fineberg N, Chamberlain SR, Stein DJ.

Pharmacotherapy for trichotillomania.

Cochrane Database of Systematic Reviews 2021, Issue 9. Art. No.: CD007662.

DOI: 10.1002/14651858.CD007662.pub3.

www.cochranelibrary.com 
TABLE OF CONTENTS

ABSTRACT

PLAIN LANGUAGE SUMMARY

SUMMARY OF FINDINGS

BACKGROUND

OBJECTIVES

METHODS

RESULTS

Figure 1.

Figure 2.

Figure 3.

DISCUSSION

AUTHORS' CONCLUSIONS

ACKNOWLEDGEMENTS

REFERENCES

CHARACTERISTICS OF STUDIES

DATA AND ANALYSES

Analysis 1.1. Comparison 1: Antioxidants versus placebo in adults, Outcome 1: Treatment response

Analysis 1.2. Comparison 1: Antioxidants versus placebo in adults, Outcome 2: Reduction of TTM symptom severity ..............

Analysis 1.3. Comparison 1: Antioxidants versus placebo in adults, Outcome 3: Reduction of comorbid symptoms of depression

Analysis 1.4. Comparison 1: Antioxidants versus placebo in adults, Outcome 4: Quality of Life - functional disability

Analysis 1.5. Comparison 1: Antioxidants versus placebo in adults, Outcome 5: Tolerability of treatment - Dropouts due to adverse events

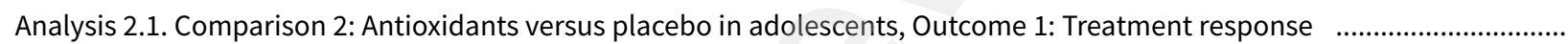
Analysis 2.2. Comparison 2: Antioxidants versus placebo in adolescents, Outcome 2: Reduction of TTM symptom severity ...... Analysis 2.3. Comparison 2: Antioxidants versus placebo in adolescents, Outcome 3: Reduction of comorbid symptoms of depression

Analysis 2.4. Comparison 2: Antioxidants versus placebo in adolescents, Outcome 4: Quality of Life - functional disability ...... Analysis 2.5. Comparison 2: Antioxidants versus placebo in adolescents, Outcome 5: Tolerability of treatment - Dropouts due to adverse events

Analysis 3.1. Comparison 3: Antipsychotics versus placebo in adults, Outcome 1: Treatment response ................................... Analysis 3.2. Comparison 3: Antipsychotics versus placebo in adults, Outcome 2: Reduction of TTM symptom severity ............ Analysis 3.3. Comparison 3: Antipsychotics versus placebo in adults, Outcome 3: Tolerability of treatment - Dropouts due to adverse events

Analysis 3.4. Comparison 3: Antipsychotics versus placebo in adults, Outcome 4: Tolerability of treatment - Common adverse events (> 20\%)

Analysis 4.1. Comparison 4: Cell signal transducers versus placebo in adults, Outcome 1: Treatment response ....................... Analysis 4.2. Comparison 4: Cell signal transducers versus placebo in adults, Outcome 2: Reduction of TTM symptom severity . Analysis 4.3. Comparison 4: Cell signal transducers versus placebo in adults, Outcome 3: Tolerability of treatment - Dropouts due to adverse events

Analysis 4.4. Comparison 4: Cell signal transducers versus placebo in adults, Outcome 4: Tolerability of treatment - Common adverse events (> 20\%)

Analysis 5.1. Comparison 5: Glutamate modulators versus placebo in adults, Outcome 1: Treatment response ........................ Analysis 5.2. Comparison 5: Glutamate modulators versus placebo in adults, Outcome 2: Reduction of TTM symptom severity . Analysis 5.3. Comparison 5: Glutamate modulators versus placebo in adults, Outcome 3: Reduction of comorbid symptoms of depression

Analysis 5.4. Comparison 5: Glutamate modulators versus placebo in adults, Outcome 4: Quality of Life - functional disability . Analysis 5.5. Comparison 5: Glutamate modulators versus placebo in adults, Outcome 5: Tolerability of treatment - Dropouts due to adverse events

Analysis 6.1. Comparison 6: Glutamate modulators versus placebo in children and adolescents, Outcome 1: Treatment response

Analysis 6.2. Comparison 6: Glutamate modulators versus placebo in children and adolescents, Outcome 2: Reduction of TTM symptom severity 
Analysis 6.3. Comparison 6: Glutamate modulators versus placebo in children and adolescents, Outcome 3: Reduction of comorbid symptoms of depression

Analysis 6.4. Comparison 6: Glutamate modulators versus placebo in children and adolescents, Outcome 4: Tolerability of treatment - Dropouts due to adverse eventsDropouts due to adverse events

Analysis 6.5. Comparison 6: Glutamate modulators versus placebo in children and adolescents, Outcome 5: Tolerability of treatment - Common adverse events (> 20\%)

Analysis 7.1. Comparison 7: Opioid antagonists versus placebo in adults, Outcome 1: Treatment response ............................. Analysis 7.2. Comparison 7: Opioid antagonists versus placebo in adults, Outcome 2: Reduction of TTM symptom severity ..... Analysis 7.3. Comparison 7: Opioid antagonists versus placebo in adults, Outcome 3: Reduction of comorbid symptoms of depression

Analysis 7.4. Comparison 7: Opioid antagonists versus placebo in adults, Outcome 4: Quality of Life - functional disability ..... Analysis 7.5. Comparison 7: Opioid antagonists versus placebo in adults, Outcome 5: Tolerability of treatment - Common adverse events (> 20\%)

Analysis 8.1. Comparison 8: SSRIs versus placebo in adults, Outcome 1: Treatment response

Analysis 8.2. Comparison 8: SSRIs versus placebo in adults, Outcome 2: Reduction of TTM symptom severity ......................... Analysis 8.3. Comparison 8: SSRIs versus placebo in adults, Outcome 3: Reduction of comorbid symptoms of depression ...... Analysis 8.4. Comparison 8: SSRIs versus placebo in adults, Outcome 4: Tolerability of treatment - Dropouts due to adverse events

Analysis 8.5. Comparison 8: SSRIs versus placebo in adults, Outcome 5: Tolerability of treatment - Common adverse events (> 20\%)

Analysis 9.1. Comparison 9: TCAs with predominantly SRI actions versus placebo in adults, Outcome 1: Treatment response ..

Analysis 9.2. Comparison 9: TCAs with predominantly SRI actions versus placebo in adults, Outcome 2: Reduction of TTM symptom severity

Analysis 9.3. Comparison 9: TCAs with predominantly SRI actions versus placebo in adults, Outcome 3: Tolerability of treatment - Dropouts due to adverse eventsDropouts due to adverse events

Analysis 9.4. Comparison 9: TCAs with predominantly SRI actions versus placebo in adults, Outcome 4: Tolerability of treatment - Common adverse events (> 20\%)

Analysis 10.1. Comparison 10: TCAs with predominantly SRI actions versus other TCAs in adults, Outcome 1: Treatment response

Analysis 10.2. Comparison 10: TCAs with predominantly SRI actions versus other TCAs in adults, Outcome 2: Reduction of TTM symptom severity

Analysis 10.3. Comparison 10: TCAs with predominantly SRI actions versus other TCAs in adults, Outcome 3: Reduction of comorbid symptoms of depression

Analysis 10.4. Comparison 10: TCAs with predominantly SRI actions versus other TCAs in adults, Outcome 4: Tolerability of treatment - Dropouts due to adverse events

Analysis 10.5. Comparison 10: TCAs with predominantly SRI actions versus other TCAs in adults, Outcome 5: Tolerability of treatment - Common adverse events (> 20\%)

APPENDICES

FEEDBACK

WHAT'S NEW

HISTORY

CONTRIBUTIONS OF AUTHORS

DECLARATIONS OF INTEREST

SOURCES OF SUPPORT

DIFFERENCES BETWEEN PROTOCOL AND REVIEW

NOTES 
[Intervention Review]

\title{
Pharmacotherapy for trichotillomania
}

Jacob Hoffman ${ }^{1}$, Taryn Williams ${ }^{1}$, Rachel Rothbart ${ }^{2}$, Jonathan C Ipser ${ }^{1}$, Naomi Fineberg 3 , Samuel R Chamberlain 4 , Dan J Stein 1

1Department of Psychiatry and Mental Health, University of Cape Town, Cape Town, South Africa. 2Department of Psychiatry, University of British Columbia, Vancouver, Canada. ${ }^{3}$ National Obsessive Compulsive Disorders Treatment Service, Queen Elizabeth II Hospital, Welwyn Garden City, UK. ${ }^{4}$ Department of Psychiatry, University of Southampton, Southampton, UK

Contact: Dan J Stein, dan.stein@uct.ac.za.

Editorial group: Cochrane Common Mental Disorders Group.

Publication status and date: New search for studies and content updated (conclusions changed), published in Issue 9, 2021.

Citation: Hoffman J, Williams T, Rothbart R, Ipser JC, Fineberg N, Chamberlain SR, Stein DJ. Pharmacotherapy for trichotillomania. Cochrane Database of Systematic Reviews 2021, Issue 9. Art. No.: CD007662. DOI: 10.1002/14651858.CD007662.pub3.

Copyright @ 2021 The Cochrane Collaboration. Published by John Wiley \& Sons, Ltd.

\begin{abstract}
A B S T R A C T
Background

Trichotillomania (TTM; hair-pulling disorder) is a prevalent and disabling disorder characterised by recurrent hair-pulling. Here we update a previous Cochrane Review on the effects of medication for TTM.
\end{abstract}

\section{Objectives}

To assess the effects of medication for trichotillomania (TTM) in adults, children and adolescents compared with placebo or other medication.

\section{Search methods}

We searched CENTRAL, MEDLINE, Embase, PsycINFO, eleven other bibliographic databases, trial registries and grey literature sources (to 26 November 2020). We checked reference lists and contacted subject experts.

\section{Selection criteria}

We selected randomised controlled trials of medication versus placebo or other medication for TTM in adults, children and adolescents.

\section{Data collection and analysis}

We used standard methodological procedures expected by Cochrane.

\section{Main results}

Twelve studies were included. We identified 10 studies in adults (286 participants) with a mean sample size of 29 participants per trial; one study in children and adolescents (39 participants); and, one study in adults and adolescents (22 participants: 18 adults and 4 adolescents). All studies were single-centre, outpatient trials. Eleven studies compared medication and placebo (334 participants); one study compared two medications (13 participants). Studies were 5 to 13 weeks duration. We undertook meta-analysis only for opioid antagonists as other comparisons contained a single study, or reported insufficient data.

\section{Antioxidants versus placebo in adults}

There was little to no difference in treatment response between antioxidant (35.7\%) and placebo groups (28.6\%) after six weeks, based on a single trial of silymarin (risk ratio (RR) 2.25, 95\% confidence interval (CI) 0.84 to 5.99; 36 participants; low-certainty evidence). We could not calculate differences in number of dropouts as there were no events in either group (18 participants; low-certainty evidence).

\section{Antioxidants versus placebo in adolescents}


There was little to no difference in treatment response between antioxidant (50\%) and placebo groups (25\%) after six weeks, based on a single trial of silymarin (RR $2.00,95 \% \mathrm{Cl} 0.28$ to $14.20 ; 8$ participants; low-certainty evidence). We could not calculate differences in number of dropouts as there were no events in either group (8 participants; low-certainty evidence).

\section{Antipsychotics versus placebo in adults}

There may be greater treatment response in the antipsychotic group (85\%) compared to the placebo group (17\%) after 12 weeks, based on a single trial of olanzapine (RR 5.08, 95\% Cl 1.4 to 18.37; 25 participants; low-certainty evidence). We could not calculate differences in number of dropouts as there were no events in either group (25 participants; low-certainty evidence).

\section{Cell signal transducers versus placebo in adults}

There was little to no difference in treatment response between cell signal transducer $(42.1 \%)$ and placebo groups (31.6\%) after 10 weeks, based on a single trial of inositol (RR $1.33,95 \% \mathrm{Cl} 0.57$ to $3.11 ; 38$ participants; low-certainty evidence). We could not calculate differences in number of dropouts as there were no events in either group (38 participants; low-certainty evidence).

\section{Glutamate modulators versus placebo in adults}

There is probably greater treatment response in the glutamate modulator group (56\%) compared to the placebo group (16\%) after 12 weeks, based on a single trial of $\mathrm{N}$-acetylcysteine (RR 3.5, 95\% Cl 1.34 to 9.17; 50 participants; moderate-certainty evidence). We could not calculate differences in number of dropouts as there were no events in either group (50 participants; low-certainty evidence).

\section{Glutamate modulators versus placebo in children and adolescents}

There was little to no difference in treatment response between the glutamate modulator (25\%) and placebo groups (21.1\%) in children and adolescents, based on a single trial of $\mathrm{N}$-acetylcysteine (RR 1.19, $95 \% \mathrm{Cl} 0.37$ to $3.77 ; 39$ participants; low-certainty evidence). There was little to no difference in dropouts due to adverse events between glutamate modulator (5\%) and placebo (0\%) groups, based on a single trial (RR 2.86, 95\% $\mathrm{Cl} 0.12$ to 66.11 ; 39 participants; low-certainty evidence).

\section{Opioid antagonists versus placebo in adults}

There may be little to no difference in treatment response between opioid antagonist (37.5\%) and placebo groups (25\%) after six to eight weeks, based on two studies of naltrexone, but the evidence is very uncertain (RR $2.14,95 \% \mathrm{Cl} 0.25$ to $18.17 ; 2$ studies, 68 participants; very low-certainty evidence). No data were available regarding dropouts due to adverse events.

\section{Selective serotonin reuptake inhibitors (SSRIs) versus placebo in adults}

There were no data available for treatment response to SSRIs. There was little to no difference in dropouts due to adverse events in the SSRI group (5.1\%) compared to the placebo group (0\%) after 6 to 12 weeks, based on two trials of fluoxetine (RR $3.00,95 \% \mathrm{Cl} 0.33$ to 27.62 ; 2 studies, 78 participants; low-certainty evidence).

\section{Tricyclic antidepressants (TCAs) with predominantly serotonin reuptake inhibitor (SRI) actions versus placebo in adults}

There may be greater treatment response in the TCAs with predominantly SRI actions group (40\%) compared to the placebo group (0\%) after nine weeks, but the evidence is very uncertain, based on a single trial of clomipramine (RR 5.73, 95\% $\mathrm{Cl} 0.36$ to 90.83 ; 16 participants; very low-certainty evidence). There may be increased dropouts due to adverse events in the TCAs with predominantly SRI actions group (30\%) compared to the placebo group (0\%), but the evidence is very uncertain (RR $4.45,95 \% \mathrm{Cl} 0.27$ to 73.81 ; 16 participants; very lowcertainty evidence).

\section{TCAs with predominantly SRI actions versus other TCAs in adults}

There may be greater treatment response in the TCAs with predominantly SRI actions group compared to the other TCAs group after five weeks, based on a single trial comparing clomipramine to desipramine (mean difference (MD) $-4.00,95 \% \mathrm{Cl}-6.13$ to $-1.87 ; 26$ participants; low-certainty evidence). We could not calculate differences in number of dropouts as there were no events in either group (26 participants; low-certainty evidence).

\section{Authors' conclusions}

There was insufficient evidence from meta-analysis to confirm or refute the efficacy of any agent or class of medication for the treatment of TTM in adults, children or adolescents. Preliminary evidence suggests there may be beneficial treatment effects for $\mathrm{N}$-acetylcysteine, clomipramine and olanzapine in adults based on four trials, albeit with relatively small sample sizes.

\section{PLAIN LANGUAGE SUMMARY}

\section{Drug treatments for chronic hair-pulling (trichotillomania)}


Trichotillomania (TTM) is a common and disabling condition characterised by repeated hair-pulling leading to hair loss. TTM can be associated with much distress and impairment. It may also be associated with other psychiatric conditions (known as comorbidities) such as depression and anxiety disorders. Researchers have proposed that medication may be useful to treat this condition.

\section{Who will be interested in this review?}

- People with TTM or other common comorbidities.

- Families and friends of people who have TTM or other common comorbidities.

- Mental health clinicians, general practitioners, psychiatrists, psychologists and pharmacists.

\section{What questions does this review aim to answer?}

- Is medication an effective treatment for TTM in adults or children and adolescents? That is, does it have the intended result?

- Does medication reduce the severity of symptoms for adults or children and adolescents with TTM?

- Does medication aid in treating symptoms of depression in adults or children and adolescents with TTM?

- Is medication effective and tolerable for people with TTM in terms of side effects?

- Does medication improve quality of life and reduce disability?

\section{Which studies were included in the review?}

- We included nine studies comparing a medication with a placebo (control substance/not an active drug) for the treatment of TTM in adults.

- We included one study comparing two different antidepressant drugs with each other for the treatment of TTM in adults.

- We included one study comparing a medication with a placebo for the treatment of TTM in children and adolescents aged 8 to 17 years.

- We included one study comparing a medication with a placebo for the treatment of TTM in adolescents and adults aged 12 to 65 years.

- A total of 298 adults were included from the 11 studies conducted in adults, and a total of 43 children and adolescents were included from the two trials conducted with participants in this age group.

\section{What does the evidence from the review tell us?}

There was insufficient evidence from analysis of individual studies or across multiple scientific studies (known as meta-analysis) to confirm or refute the effectiveness of any specific agent or class of medication for the treatment of TTM in adults, children or adolescents. In adults, evidence suggests tricyclic antidepressants (TCAs; a type of antidepressant) with predominantly serotonin reuptake inhibitor (SRI; increasing serotonin levels in the brain) actions may show a beneficial treatment effect compared to other TCAs, with reduction in TTM symptom severity. However, certainty in the estimate of effect was low and is based on a single trial comparing clomipramine with desipramine. Antipsychotics in adults may show a beneficial treatment effect and possible reduction of TTM symptom severity, with low-certainty in the estimate of effect, based on a single trial of olanzapine. Glutamate modulators (a type of amino acid modulator) in adults showed a probable beneficial treatment effect and a likely reduction in TTM symptom severity, with moderate-certainty in the estimate of effect, although based on a single trial of $\mathrm{N}$-acetylcysteine (NAC; a glutamate modulator). Glutamate modulators in children and adolescents (8 to 17 years old) showed no evidence for beneficial effect in terms of the percentage of participants responding to treatment in a single study of NAC. However, evidence suggests a potential large reduction in TTM symptom severity; however, with low-certainty in the estimate. There was little to no evidence for beneficial treatment effects in terms of the percentage of participants responding to treatment or reduction of TTM symptom severity reported for antioxidants, cell signal transducers, opioid antagonists or selective serotonin reuptake inhibitors (SSRIs; a type of antidepressant) in adults, children or adolescents.

Attrition due to adverse events was only reported for SSRIs and TCAs with predominantly SRI actions in adults and for glutamate modulators in children and adolescents. Glutamate modulators had the least severe side effect profile in adults, while antipsychotics were associated with several adverse side effects, although with low-certainty in the effect estimate, and based on individual trials for each medication class. There was low-certainty evidence showing no difference in dropouts due to adverse events between the glutamate modulator group and placebo group in the single study exclusively in children and adolescents.

\section{What should happen next?}

There is some evidence that NAC (a glutamate modulator) probably demonstrates efficacy in TTM in adults and possible symptom severity reduction in children and adolescents. There is some evidence that olanzapine (an antipsychotic) and clomipramine (a TCA with predominantly SRI actions) may demonstrate efficacy in TTM in adults, although based on individual trials and therefore not generalisable to other agents in the same medication classes. Studies are few and sample sizes are small, and as a result it is not possible to draw 
high-quality conclusions from meta-analysis. Additional studies, with rigorous designs and appropriately powered samples are needed, particularly in children and adolescents. Future studies could also include people with common comorbidities, as the current evidence base may not be representative of clinical populations, who might have multiple medical and psychiatric conditions. 


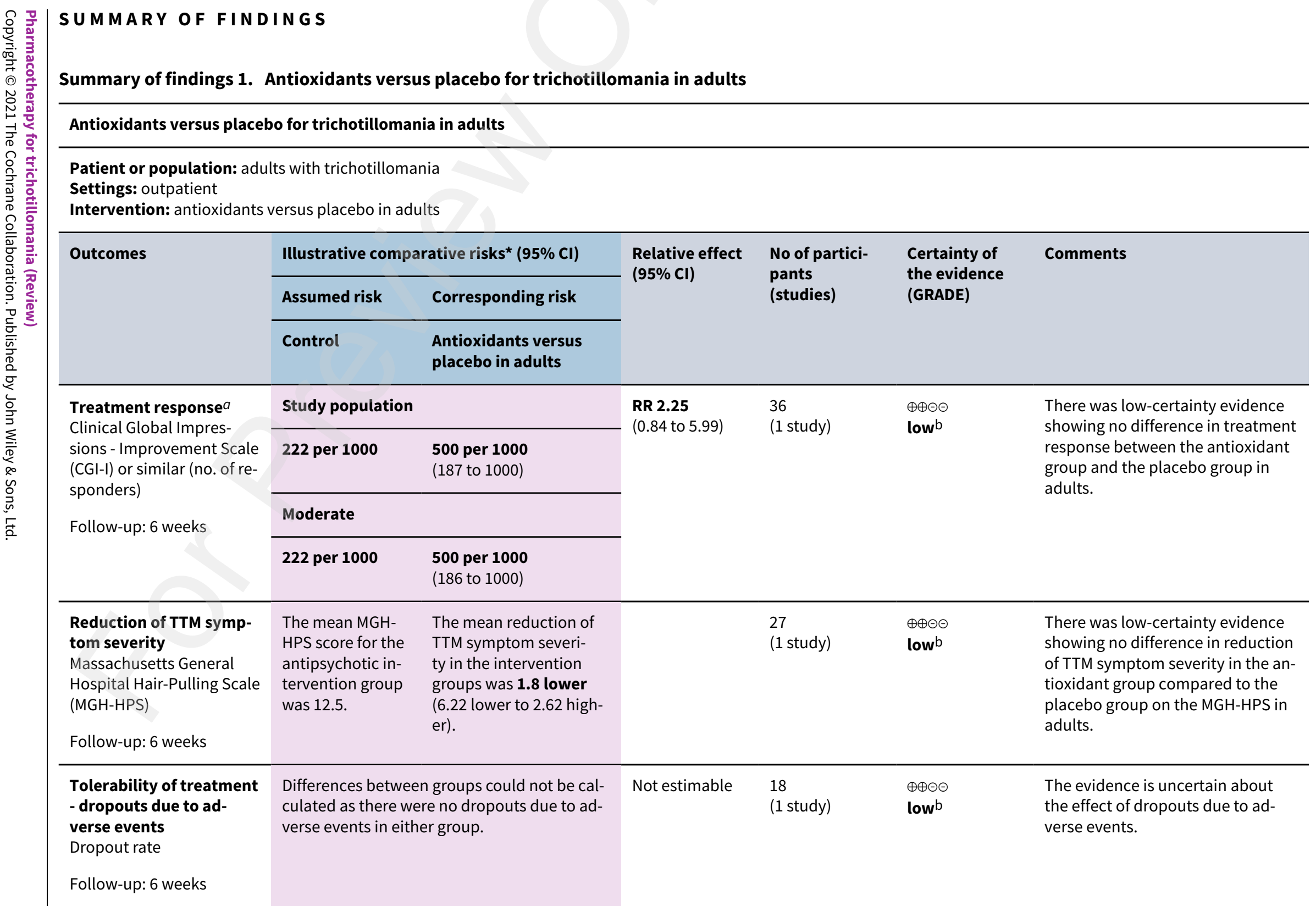

${ }^{\star}$ The basis for the assumed risk (e.g. the median control group risk across studies) is provided in footnotes. The corresponding risk (and its $95 \%$ confidence interval) is based on the assumed risk in the comparison group and the relative effect of the intervention (and its $95 \% \mathrm{Cl}$ ). 
GRADE Working Group grades of evidence

High certainty: further research is very unlikely to change our confidence in the estimate of effect.

Moderate certainty: further research is likely to have an important impact on our confidence in the estimate of effect and may change the estimate.

Low certainty: further research is very likely to have an important impact on our confidence in the estimate of effect and is likely to change the estimate.

Very low certainty: we are very uncertain about the estimate.

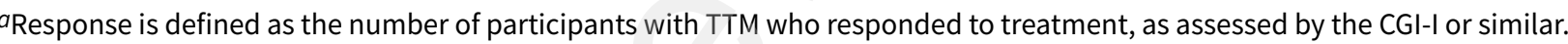

bDowngraded by two levels due to wide confidence interval and very small sample size $(<50)$

\section{Summary of findings 2 . Antioxidants versus placebo for trichotillomania in adolescents}

\section{Antioxidants versus placebo for trichotillomania in adolescents}

Patient or population: children with trichotillomania

Settings: outpatient

Intervention: antioxidants versus placebo in adolescents

\begin{tabular}{|c|c|c|c|c|c|c|}
\hline \multirow[t]{3}{*}{ Outcomes } & \multicolumn{2}{|c|}{ Illustrative comparative risks ${ }^{\star}(95 \% \mathrm{CI})$} & \multirow{3}{*}{$\begin{array}{l}\text { Relative effect } \\
(95 \% \mathrm{Cl})\end{array}$} & \multirow{3}{*}{$\begin{array}{l}\text { No of partici- } \\
\text { pants } \\
\text { (studies) }\end{array}$} & \multirow{3}{*}{$\begin{array}{l}\text { Certainty of } \\
\text { the evidence } \\
\text { (GRADE) }\end{array}$} & \multirow[t]{3}{*}{ Comments } \\
\hline & Assumed risk & Corresponding risk & & & & \\
\hline & Control & $\begin{array}{l}\text { Antioxidants versus } \\
\text { placebo in adolescents }\end{array}$ & & & & \\
\hline \multirow{4}{*}{$\begin{array}{l}\text { Treatment response } a \\
\text { Clinical Global Impres- } \\
\text { sions - Improvement Scale } \\
\text { (CGI-I) or similar (no. of re- } \\
\text { sponders) } \\
\text { Follow-up: } 6 \text { weeks }\end{array}$} & Study population & & \multirow{4}{*}{$\begin{array}{l}\text { RR } 2 \\
\text { (0.28 to } 14.2 \text { ) }\end{array}$} & \multirow{4}{*}{$\begin{array}{l}8 \\
\text { (1 study) }\end{array}$} & \multirow{4}{*}{$\begin{array}{l}\oplus \oplus \odot \ominus \\
\text { low }^{b}\end{array}$} & \multirow{4}{*}{$\begin{array}{l}\text { There was low-certainty evidence } \\
\text { showing no difference in treatment } \\
\text { response between the antioxidant } \\
\text { group and the placebo group in } \\
\text { adolescents. }\end{array}$} \\
\hline & 250 per 1000 & $\begin{array}{l}\mathbf{5 0 0} \text { per } \mathbf{1 0 0 0} \\
(70 \text { to } 1000)\end{array}$ & & & & \\
\hline & \multicolumn{2}{|l|}{ Moderate } & & & & \\
\hline & 333 per 1000 & $\begin{array}{l}\mathbf{6 6 6} \text { per } 1000 \\
(110 \text { to } 1000)\end{array}$ & & & & \\
\hline $\begin{array}{l}\text { Reduction of TTM symp- } \\
\text { tom severity } \\
\text { Massachusetts General } \\
\text { Hospital Hair-Pulling Scale } \\
\text { (MGH-HPS) } \\
\text { Follow-up: } 6 \text { weeks }\end{array}$ & $\begin{array}{l}\text { The mean MGH- } \\
\text { HPS score for the } \\
\text { antipsychotic in- } \\
\text { tervention group } \\
\text { was } 6.83 \text {. }\end{array}$ & $\begin{array}{l}\text { The mean reduction of } \\
\text { TTM symptom severi- } \\
\text { ty in the intervention } \\
\text { groups was } \\
\mathbf{5 . 8 7} \text { lower } \\
\text { (17.46 lower to } 5.72 \\
\text { higher). }\end{array}$ & & $\begin{array}{l}6 \\
\text { (1 study) }\end{array}$ & $\begin{array}{l}\oplus \oplus \odot \ominus \\
\text { low }^{b}\end{array}$ & $\begin{array}{l}\text { There was low-certainty evidence } \\
\text { showing no difference in reduction } \\
\text { of TTM symptom severity in the an- } \\
\text { tioxidant group compared to the } \\
\text { placebo group on the MGH-HPS in } \\
\text { adolescents. }\end{array}$ \\
\hline
\end{tabular}


Tolerability of treatment - dropouts due to ad-

verse events

Differences between groups could not be calculated as there were no dropouts due to ad-

Not estimable
The evidence is uncertain about the effect of dropouts due to adverse events.

Follow-up: 6 weeks

*The basis for the assumed risk (e.g. the median control group risk across studies) is provided in footnotes. The corresponding risk (and its $95 \%$ confidence interval) is based on the assumed risk in the comparison group and the relative effect of the intervention (and its $95 \% \mathrm{Cl}$ ).

Cl: confidence interval; RR: risk ratio

GRADE Working Group grades of evidence

High certainty: further research is very unlikely to change our confidence in the estimate of effect.

Moderate certainty: further research is likely to have an important impact on our confidence in the estimate of effect and may change the estimate.

Low certainty: further research is very likely to have an important impact on our confidence in the estimate of effect and is likely to change the estimate.

Very low certainty: we are very uncertain about the estimate.

a Response is defined as the number of participants with TTM who responded to treatment, as assessed by the CGI-I or similar.

bDowngraded by two levels due to wide confidence interval and very small sample size $(<50)$.

Summary of findings 3. Antipsychotics versus placebo for trichotillomania in adults

\section{Antipsychotics versus placebo for trichotillomania in adults}

Patient or population: adults with trichotillomania

Settings: outpatient

Intervention: antipsychotics

Comparison: placebo

\begin{tabular}{|c|c|c|c|c|c|c|}
\hline \multirow[t]{2}{*}{ Outcomes } & \multicolumn{2}{|c|}{ Illustrative comparative risks $(95 \% \mathrm{CI})$} & \multirow{2}{*}{$\begin{array}{l}\text { Relative effect } \\
(95 \% \mathrm{CI})\end{array}$} & \multirow{2}{*}{$\begin{array}{l}\text { No of partici- } \\
\text { pants } \\
\text { (studies) }\end{array}$} & \multirow{2}{*}{$\begin{array}{l}\text { Certainty of } \\
\text { the evidence } \\
\text { (GRADE) }\end{array}$} & \multirow[t]{2}{*}{ Comments } \\
\hline & Assumed risk & Corresponding risk & & & & \\
\hline \multirow{2}{*}{$\begin{array}{l}\text { Treatment response } a \\
\text { Clinical Global Impres- } \\
\text { sions - Improvement Scale } \\
\text { (CGI-I) } \\
\text { (no. of responders) }\end{array}$} & \multicolumn{2}{|c|}{ Study population } & \multirow{2}{*}{$\begin{array}{l}\text { RR } 5.08 \\
\text { (1.4 to } 18.37 \text { ) }\end{array}$} & \multirow{2}{*}{$\begin{array}{l}25 \\
\text { (1 study) }\end{array}$} & \multirow{2}{*}{$\begin{array}{l}\oplus \oplus \ominus \ominus \\
\text { low }^{b}\end{array}$} & \multirow{2}{*}{$\begin{array}{l}\text { There was low-certainty evi- } \\
\text { dence of benefit on the number } \\
\text { of participants who respond- } \\
\text { ed to treatment in the antipsy- } \\
\text { chotic group compared to the } \\
\text { placebo group. }\end{array}$} \\
\hline & \multicolumn{2}{|l|}{ Moderate } & & & & \\
\hline
\end{tabular}




\section{Reduction of TTM symp-}

tom severity

The mean MGH- The mean reduction of

HPS score for the TTM symptom severity

Massachusetts General

antipsychotic inter-

in the int

There was low-certainty evidence for a reduction of TTM

symptom severity in the an-

Hospital Hair-Pulling Scale 8.38 .

4.87 points lower tipsychotic group compared to the placebo group on the MGH(MGH-HPS)

Follow-up: 12 weeks

Tolerability of treatment - dropouts due to ad-

verse events

Differences between groups could not be calculated as there were no dropouts due to adverse events in either group.
Not estimable

25

( 1 study) $\oplus \oplus \ominus \ominus$

lowc
The evidence was uncertain about the effect of dropouts due to adverse events.

Dropout rate
Follow-up: 12 weeks

*The basis for the assumed risk (e.g. the median control group risk across studies) is provided in footnotes. The corresponding risk (and its $95 \%$ confidence interval) is based on the assumed risk in the comparison group and the relative effect of the intervention (and its $95 \% \mathrm{Cl}$ ).

Cl: confidence interval; RR: risk ratio

GRADE Working Group grades of evidence

High certainty: further research is very unlikely to change our confidence in the estimate of effect.

Moderate certainty: further research is likely to have an important impact on our confidence in the estimate of effect and may change the estimate.

Low certainty: further research is very likely to have an important impact on our confidence in the estimate of effect and is likely to change the estimate.

Very low certainty: we are very uncertain about the estimate.

aResponse is defined as the number of participants with TTM who responded to treatment, as assessed by the CGI-I or similar.

bDowngraded by two levels due to very small sample size $(<50)$.

cDowngraded by two levels due to zero events and very small sample size $(<50)$.

\section{Summary of findings 4. Cell signal transducers versus placebo for trichotillomania in adults}

\section{Cell signal transducers versus placebo for trichotillomania in adults}

Patient or population: adults with trichotillomania

Settings: outpatient

Intervention: cell signal transducers

Comparison: placebo

\begin{tabular}{lllll}
\hline Outcomes & Illustrative comparative risks ${ }^{\star}(95 \% \mathrm{CI})$ & $\begin{array}{l}\text { Relative effect } \\
(95 \% \mathrm{CI})\end{array}$ & $\begin{array}{l}\text { No of partici- } \\
\text { pants }\end{array}$ & $\begin{array}{l}\text { Certainty of } \\
\text { the evidence }\end{array}$
\end{tabular}




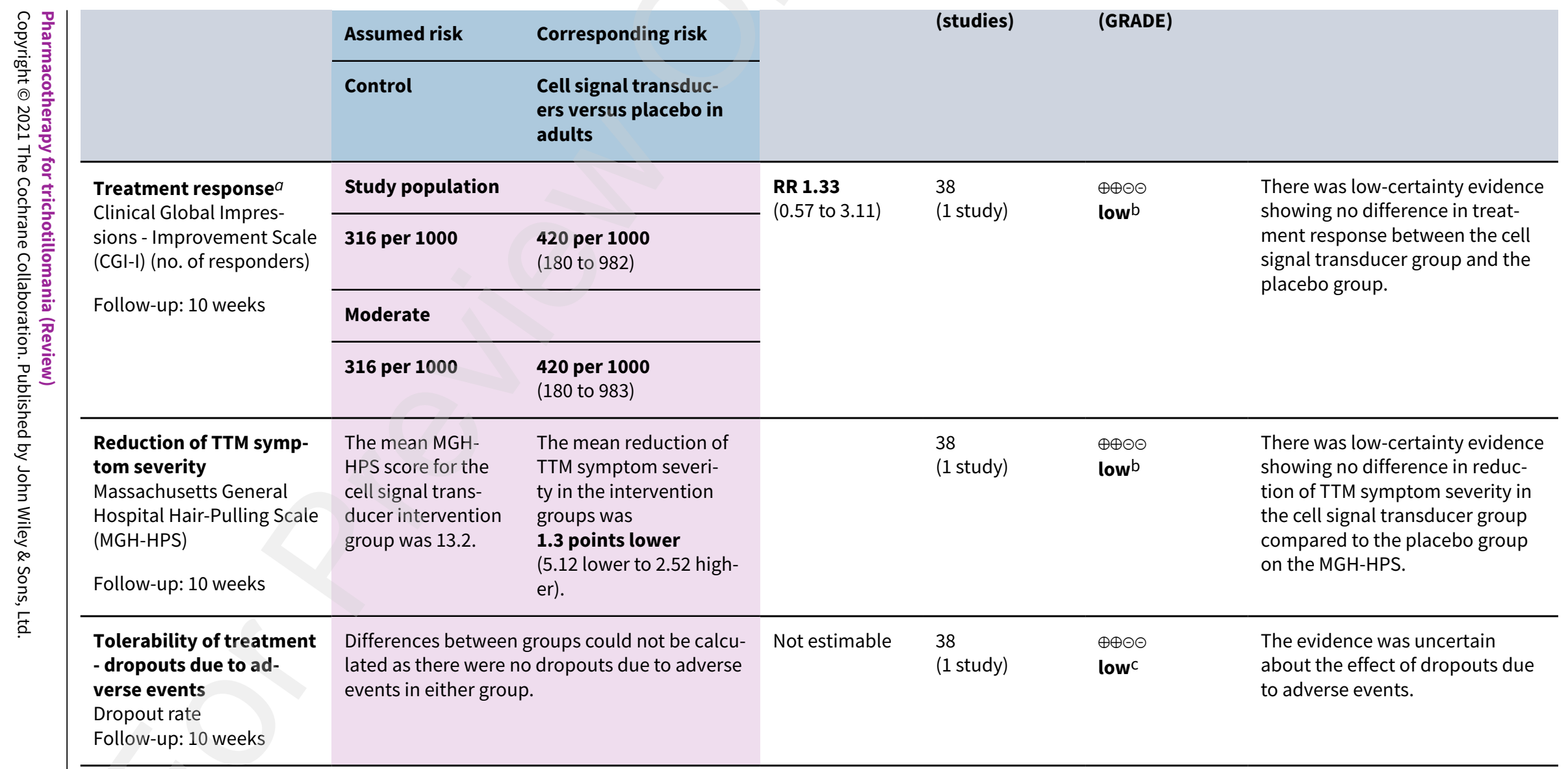

*The basis for the assumed risk (e.g. the median control group risk across studies) is provided in footnotes. The corresponding risk (and its $95 \%$ confidence interval) is based on the assumed risk in the comparison group and the relative effect of the intervention (and its $95 \% \mathrm{Cl}$ ).

Cl: confidence interval; RR: risk ratio

GRADE Working Group grades of evidence

High certainty: further research is very unlikely to change our confidence in the estimate of effect.

Moderate certainty: further research is likely to have an important impact on our confidence in the estimate of effect and may change the estimate.

Low certainty: further research is very likely to have an important impact on our confidence in the estimate of effect and is likely to change the estimate.

Very low certainty: we are very uncertain about the estimate.

a Response is defined as the number of participants with TTM who responded to treatment, as assessed by the CGI-I or similar.

bDowngraded by two levels due to wide confidence interval and very small sample size $(<50)$.

cDowngraded by two levels due to zero events and very small sample size $(<50)$. 


\section{Glutamate modulators versus placebo for trichotillomania in adults}

Patient or population: adults with trichotillomania

Settings: outpatient

Intervention: glutamate modulators

Comparison: placebo

\begin{tabular}{|c|c|c|c|c|c|c|}
\hline \multirow[t]{3}{*}{ Outcomes } & \multicolumn{2}{|c|}{ Illustrative comparative risks* $(95 \% \mathrm{CI})$} & \multirow{3}{*}{$\begin{array}{l}\text { Relative effect } \\
(95 \% \mathrm{Cl})\end{array}$} & \multirow{3}{*}{$\begin{array}{l}\text { No of partici- } \\
\text { pants } \\
\text { (studies) }\end{array}$} & \multirow{3}{*}{$\begin{array}{l}\text { Certainty of } \\
\text { the evidence } \\
\text { (GRADE) }\end{array}$} & \multirow[t]{3}{*}{ Comments } \\
\hline & Assumed risk & Corresponding risk & & & & \\
\hline & Control & $\begin{array}{l}\text { Glutamate modula- } \\
\text { tors versus placebo in } \\
\text { adults }\end{array}$ & & & & \\
\hline \multirow{4}{*}{$\begin{array}{l}\text { Treatment response } \\
\text { Clinical Global Impres- } \\
\text { sions - Improvement } \\
\text { Scale (CGI-I) (no. of re- } \\
\text { sponders) } \\
\text { Follow-up: } 12 \text { weeks }\end{array}$} & Study population & & \multirow{4}{*}{$\begin{array}{l}\text { RR } 3.5 \\
\text { (1.34 to 9.17) }\end{array}$} & \multirow{4}{*}{$\begin{array}{l}50 \\
\text { (1 study) }\end{array}$} & \multirow{4}{*}{$\begin{array}{l}\oplus \oplus \oplus \ominus \\
\text { moderate }^{b}\end{array}$} & \multirow{4}{*}{$\begin{array}{l}\text { There was moderate-certainty ev- } \\
\text { idence of benefit on the number } \\
\text { of participants who responded to } \\
\text { treatment in the glutamate modula- } \\
\text { tor group compared to the placebo } \\
\text { group in adults. }\end{array}$} \\
\hline & 160 per 1000 & $\begin{array}{l}\mathbf{5 6 0} \text { per } \mathbf{1 0 0 0} \\
(214 \text { to } 1000)\end{array}$ & & & & \\
\hline & \multicolumn{2}{|l|}{ Moderate } & & & & \\
\hline & 160 per 1000 & $\begin{array}{l}\mathbf{5 6 0} \text { per } \mathbf{1 0 0 0} \\
\text { (214 to } 1000)\end{array}$ & & & & \\
\hline $\begin{array}{l}\text { Reduction of TTM } \\
\text { symptom severity } \\
\text { Massachusetts Gener- } \\
\text { al Hospital Hair-Pulling } \\
\text { Scale (MGH-HPS) } \\
\text { Follow-up: } 12 \text { weeks }\end{array}$ & $\begin{array}{l}\text { The mean MGH- } \\
\text { HPS score for the } \\
\text { glutamate modu- } \\
\text { lator intervention } \\
\text { group was } 10.4 \text {. }\end{array}$ & $\begin{array}{l}\text { The mean reduction of } \\
\text { TTM symptom severi- } \\
\text { ty in the intervention } \\
\text { groups was } \\
\mathbf{5 . 6} \text { points lower } \\
\text { (8.5 to } 2.7 \text { lower). }\end{array}$ & & $\begin{array}{l}50 \\
\text { (1 study) }\end{array}$ & $\begin{array}{l}\oplus \oplus \oplus \ominus \\
\text { moderate }^{b}\end{array}$ & $\begin{array}{l}\text { There was moderate-certainty evi- } \\
\text { dence for a reduction of TTM symp- } \\
\text { tom severity in the glutamate modu- } \\
\text { lator group compared to the placebo } \\
\text { group on the MGH-HPS in adults. }\end{array}$ \\
\hline $\begin{array}{l}\text { Tolerability of treat- } \\
\text { ment - dropouts due to } \\
\text { adverse events } \\
\text { Dropout rate }\end{array}$ & \multicolumn{2}{|c|}{$\begin{array}{l}\text { Differences between groups could not be cal- } \\
\text { culated as there were no dropouts due to ad- } \\
\text { verse events in either group. }\end{array}$} & Not estimable & $\begin{array}{l}50 \\
\text { (1 study) }\end{array}$ & $\begin{array}{l}\oplus \oplus \odot \ominus \\
\text { low }^{c}\end{array}$ & $\begin{array}{l}\text { The evidence was uncertain about } \\
\text { the effect of dropouts due to adverse } \\
\text { events in adults. }\end{array}$ \\
\hline
\end{tabular}

Dropout rate

Follow-up: 12 weeks

*The basis for the assumed risk (e.g. the median control group risk across studies) is provided in footnotes. The corresponding risk (and its $95 \%$ confidence interval) is based on the assumed risk in the comparison group and the relative effect of the intervention (and its $95 \% \mathrm{Cl}$ ). 
GRADE Working Group grades of evidence

High certainty: further research is very unlikely to change our confidence in the estimate of effect.

Moderate certainty: further research is likely to have an important impact on our confidence in the estimate of effect and may change the estimate.

Low certainty: further research is very likely to have an important impact on our confidence in the estimate of effect and is likely to change the estimate.

Very low certainty: we are very uncertain about the estimate.

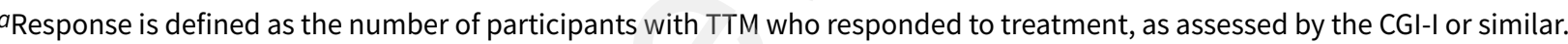

bDowngraded by one level due to small sample size (50 to 400 ).

cDowngraded by two levels due to zero events and small sample size (50 to 400 ).

\section{Summary of findings 6 . Glutamate modulators versus placebo for trichotillomania in children and adolescents}

Glutamate modulators versus placebo for trichotillomania in children and adolescents

Patient or population: children and adolescents with trichotillomania

Settings: outpatient

Intervention: glutamate modulators

Comparison: placebo

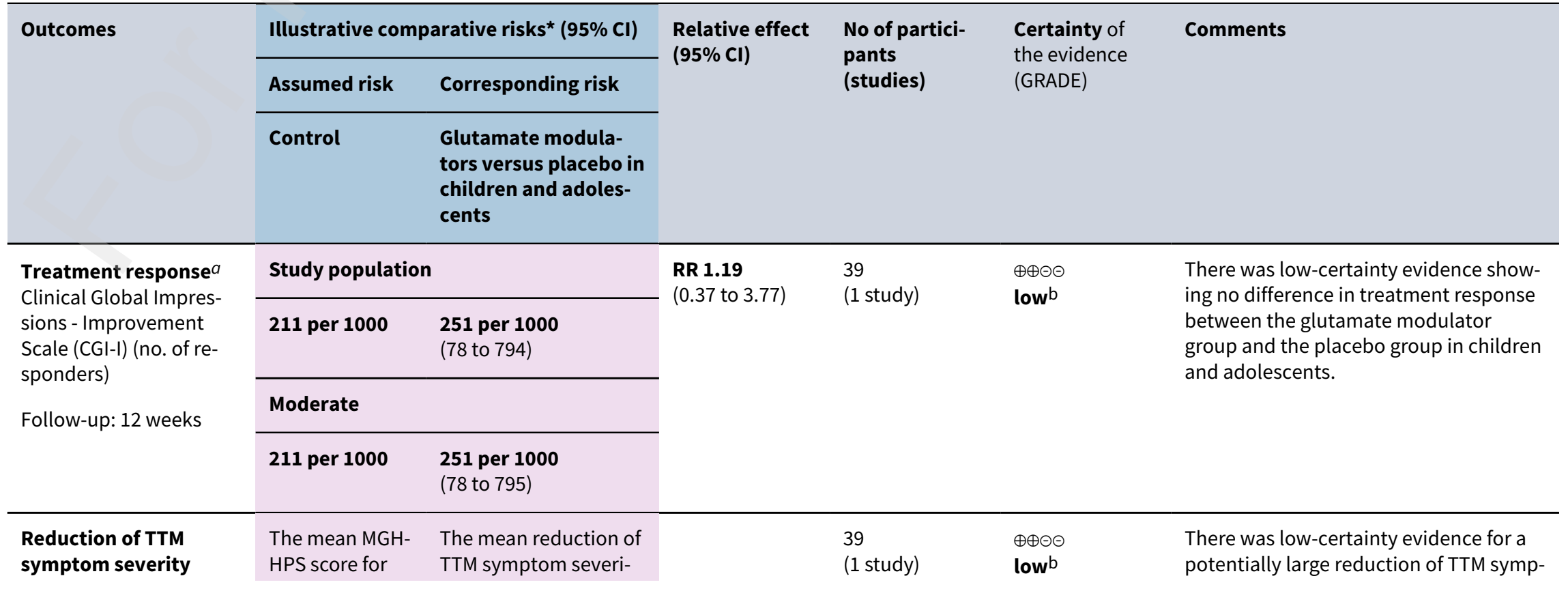




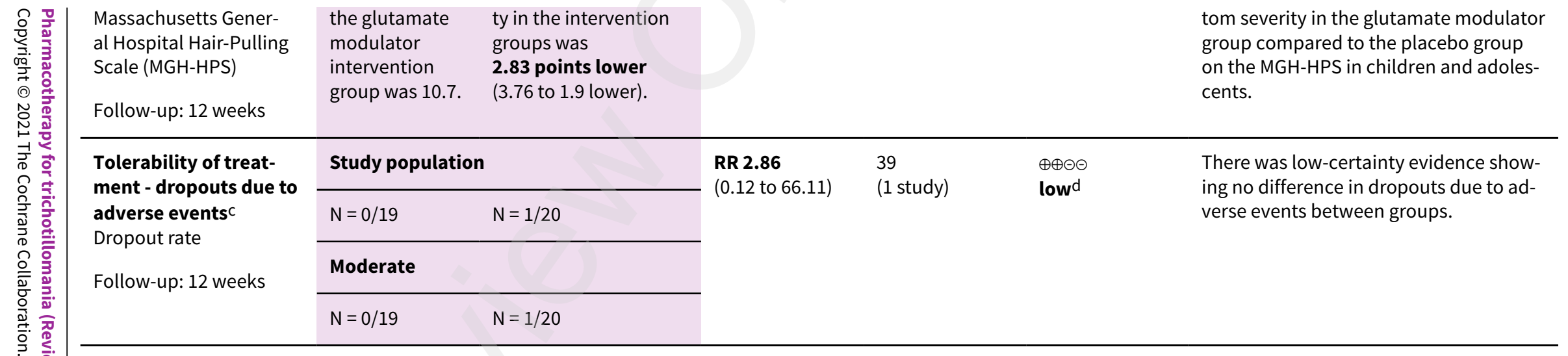

${ }^{\star}$ The basis for the assumed risk (e.g. the median control group risk across studies) is provided in footnotes. The corresponding risk (and its $95 \%$ confidence interval) is based on the assumed risk in the comparison group and the relative effect of the intervention (and its $95 \% \mathrm{Cl}$ ).

Cl: confidence interval; RR: risk ratio

GRADE Working Group grades of evidence

High certainty: further research is very unlikely to change our confidence in the estimate of effect.

Moderate certainty: further research is likely to have an important impact on our confidence in the estimate of effect and may change the estimate.

Low certainty: further research is very likely to have an important impact on our confidence in the estimate of effect and is likely to change the estimate.

Very low certainty: we are very uncertain about the estimate.

aResponse is defined as the number of participants with TTM who responded to treatment, as assessed by the CGI-I or similar.

bDowngraded by two levels due to very small sample size $(<50)$.

cNumbers presented as raw values for $\mathrm{N}$ (events) in study population (intervention and control groups), and not modelled on RR.

dDowngraded by two levels due to wide confidence interval and very small sample size $(<50)$.

\section{Summary of findings 7. Opioid antagonists versus placebo for trichotillomania in adults}

Opioid antagonists versus placebo for trichotillomania in adults

Patient or population: adults with trichotillomania

Settings: outpatient

Intervention: opioid antagonists

Comparison: placebo

\begin{tabular}{|c|c|c|c|c|c|c|}
\hline \multirow[t]{2}{*}{ Outcomes } & \multicolumn{2}{|c|}{ Illustrative comparative risks ${ }^{\star}(95 \% \mathrm{Cl})$} & \multirow{2}{*}{$\begin{array}{l}\text { Relative effect } \\
(95 \% \mathrm{CI})\end{array}$} & \multirow{2}{*}{$\begin{array}{l}\text { No of partici- } \\
\text { pants } \\
\text { (studies) }\end{array}$} & \multirow{2}{*}{$\begin{array}{l}\text { Certainty of } \\
\text { the evidence } \\
\text { (GRADE) }\end{array}$} & \multirow[t]{2}{*}{ Comments } \\
\hline & Assumed risk & Corresponding risk & & & & \\
\hline
\end{tabular}




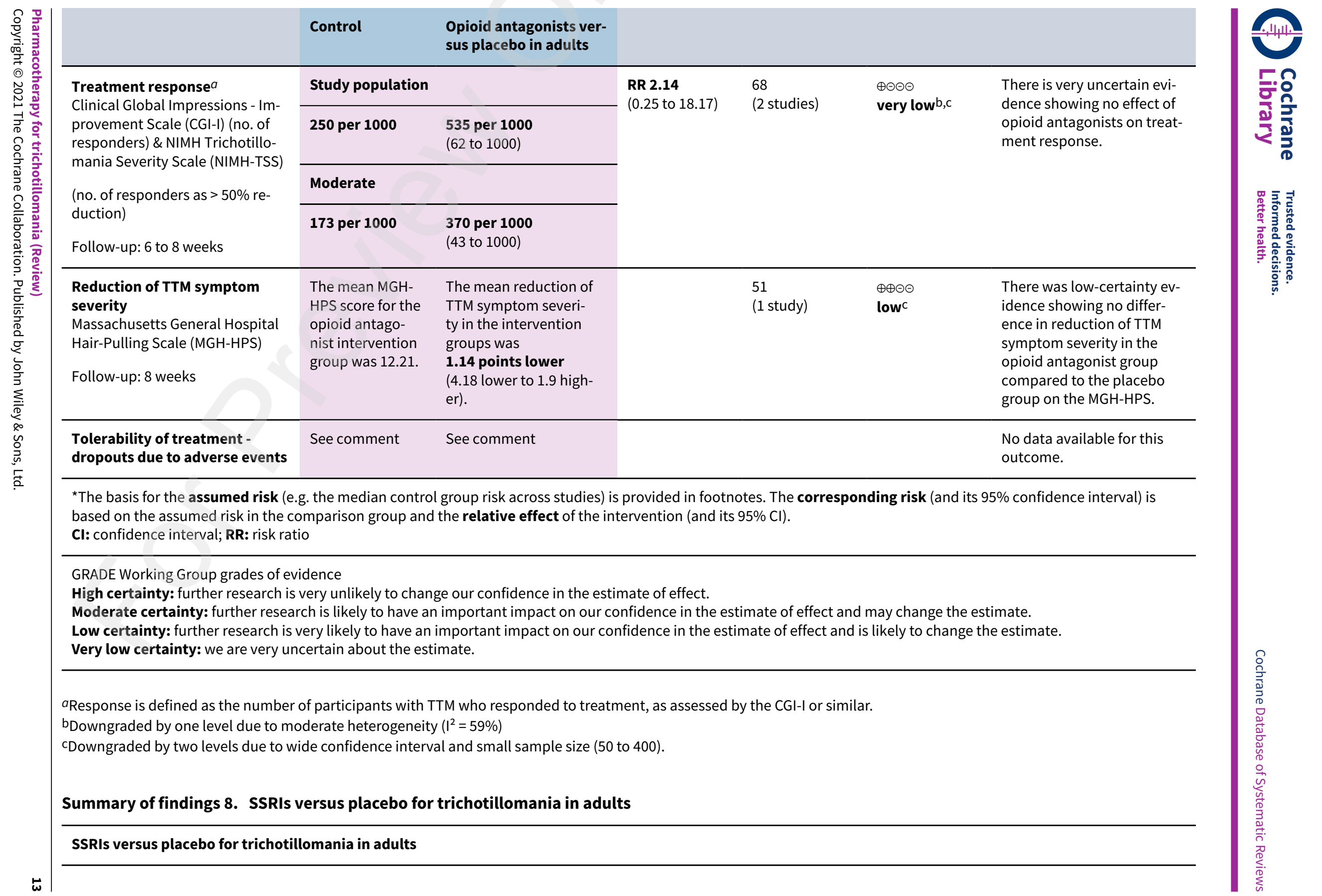


Patient or population: adults with trichotillomania

Settings: outpatient

Intervention: SSRIS

Comparison: placebo

\begin{tabular}{|c|c|c|c|c|c|c|}
\hline \multirow[t]{3}{*}{ Outcomes } & \multicolumn{2}{|c|}{ Illustrative comparative risks* $(95 \% \mathrm{Cl})$} & \multirow{3}{*}{$\begin{array}{l}\text { Relative effect } \\
(95 \% \mathrm{CI})\end{array}$} & \multirow{3}{*}{$\begin{array}{l}\text { No of partici- } \\
\text { pants } \\
\text { (studies) }\end{array}$} & \multirow{3}{*}{$\begin{array}{l}\text { Certainty of } \\
\text { the evidence } \\
\text { (GRADE) }\end{array}$} & \multirow[t]{3}{*}{ Comments } \\
\hline & Assumed risk & Corresponding risk & & & & \\
\hline & Control & $\begin{array}{l}\text { SSRIs versus placebo in } \\
\text { adults }\end{array}$ & & & & \\
\hline Treatment response & See comment & See comment & & & & $\begin{array}{l}\text { No data available for this out- } \\
\text { come. }\end{array}$ \\
\hline $\begin{array}{l}\text { Reduction of TTM symptom } \\
\text { severity } a \\
\text { Massachusetts General Hos- } \\
\text { pital Hair-Pulling Scale (MGH- } \\
\text { HPS) mean change score } \\
\text { Follow-up: } 12 \text { weeks }\end{array}$ & $\begin{array}{l}\text { The mean change } \\
\text { of the MGH-HPS } \\
\text { score for the SSRI } \\
\text { intervention group } \\
\text { was }-1.5333 \text {. }\end{array}$ & $\begin{array}{l}\text { The mean reduction of } \\
\text { TTM symptom severi- } \\
\text { ty in the intervention } \\
\text { groups was } \\
\mathbf{0 . 4 7} \text { points lower } \\
\text { (4.3 lower to } 3.36 \text { high- } \\
\text { er). }\end{array}$ & & $\begin{array}{l}31 \\
\text { (1 study) }\end{array}$ & $\begin{array}{l}\oplus \ominus \ominus \ominus \\
\text { very low } b, c, d\end{array}$ & $\begin{array}{l}\text { The evidence is very uncer- } \\
\text { tain about the effect of SSRIs } \\
\text { on reduction of TTM symp- } \\
\text { tom severity on the MGH-HPS. }\end{array}$ \\
\hline \multirow{2}{*}{$\begin{array}{l}\text { Tolerability of treatment } \\
\text { - dropouts due to adverse } \\
\text { eventse } \\
\text { Dropout rate }\end{array}$} & \multicolumn{2}{|l|}{ Study population } & \multirow{4}{*}{$\begin{array}{l}\text { RR } 3 \\
(0.33 \text { to } 27.62)\end{array}$} & \multirow{4}{*}{$\begin{array}{l}78 \\
\text { (2 studies) }\end{array}$} & \multirow{4}{*}{$\begin{array}{l}\oplus \oplus \ominus \ominus \\
\text { low }^{f}\end{array}$} & \multirow{4}{*}{$\begin{array}{l}\text { There was low-certainty evi- } \\
\text { dence showing no difference } \\
\text { in dropouts due to adverse } \\
\text { events between groups. }\end{array}$} \\
\hline & $N=0 / 39$ & $N=2 / 39$ & & & & \\
\hline \multirow[t]{2}{*}{ Follow-up: 6 to 12 weeks } & \multicolumn{2}{|l|}{ Moderate } & & & & \\
\hline & $N=0 / 39$ & $N=2 / 39$ & & & & \\
\hline
\end{tabular}

*The basis for the assumed risk (e.g. the median control group risk across studies) is provided in footnotes. The corresponding risk (and its $95 \%$ confidence interval) is based on the assumed risk in the comparison group and the relative effect of the intervention (and its $95 \% \mathrm{Cl}$ ).

Cl: confidence interval; RR: risk ratio

GRADE Working Group grades of evidence

High certainty: further research is very unlikely to change our confidence in the estimate of effect.

Moderate certainty: further research is likely to have an important impact on our confidence in the estimate of effect and may change the estimate.

Low certainty: further research is very likely to have an important impact on our confidence in the estimate of effect and is likely to change the estimate.

Very low certainty: we are very uncertain about the estimate.

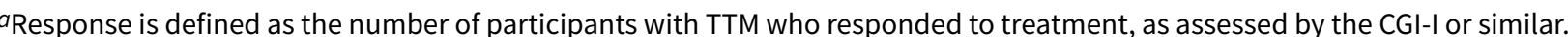

bDowngraded by one level due to serious risk of bias. Concerns with attrition bias. Intention-to-treat analysis not used. No reasons for attrition given. 
cDowngraded by one level as mean and standard deviations at endpoint were unavailable. Data provided from correspondence were mean change scores from baseline to endpoint.

dDowngraded by two levels due to wide confidence interval and very small sample size $(<50)$.

eNumbers presented as raw values for $\mathrm{N}$ (events) in study population (intervention and control groups), and not modelled on RR.

fDowngraded by two levels due to wide confidence interval and small sample size (50 to 400 ).

\section{Summary of findings 9. TCAs with predominantly SRI actions versus placebo for trichotillomania in adults}

TCAs with predominantly SRI actions versus placebo for trichotillomania in adults

Patient or population: adults with trichotillomania

Settings: outpatient

Intervention: TCAs with predominantly SRI actions

Comparison: placebo

\begin{tabular}{|c|c|c|c|c|c|c|}
\hline \multirow[t]{3}{*}{ Outcomes } & \multicolumn{2}{|c|}{ Illustrative comparative risks* $(95 \% \mathrm{Cl})$} & \multirow{3}{*}{$\begin{array}{l}\text { Relative effect } \\
(95 \% \mathrm{Cl})\end{array}$} & \multirow{3}{*}{$\begin{array}{l}\text { No of partici- } \\
\text { pants } \\
\text { (studies) }\end{array}$} & \multirow{3}{*}{$\begin{array}{l}\text { Certainty of } \\
\text { the evidence } \\
\text { (GRADE) }\end{array}$} & \multirow[t]{3}{*}{ Comments } \\
\hline & Assumed risk & Corresponding risk & & & & \\
\hline & Control & $\begin{array}{l}\text { TCAs with predomi- } \\
\text { nantly SRI actions ver- } \\
\text { sus placebo in adults }\end{array}$ & & & & \\
\hline \multirow{2}{*}{$\begin{array}{l}\text { Treatment response }{ }^{a} \\
\text { Clinical Global Impres- } \\
\text { sions - Improvement Scale } \\
\text { (CG-I) (no. of responders) }\end{array}$} & \multicolumn{2}{|l|}{ Study population } & \multirow{4}{*}{$\begin{array}{l}\text { RR } \mathbf{5 . 7 3} \\
(0.36 \text { to } 90.83)\end{array}$} & \multirow{4}{*}{$\begin{array}{l}16 \\
\text { (1 study) }\end{array}$} & \multirow{4}{*}{$\begin{array}{l}\oplus \odot \ominus \ominus \\
\text { very low } b, c\end{array}$} & \multirow{4}{*}{$\begin{array}{l}\text { The evidence is very uncertain } \\
\text { about the effect of TCAs with pre- } \\
\text { dominantly SRI actions on treat- } \\
\text { ment response. }\end{array}$} \\
\hline & 0 per 1000 & $\begin{array}{l}\text { O per } 1000 \\
(0 \text { to } 0)\end{array}$ & & & & \\
\hline \multirow[t]{2}{*}{ Follow-up: 9 weeks } & \multicolumn{2}{|l|}{ Moderate } & & & & \\
\hline & 0 per 1000 & $\begin{array}{l}\mathbf{0} \text { per } 1000 \\
(0 \text { to } 0)\end{array}$ & & & & \\
\hline \multirow[t]{2}{*}{$\begin{array}{l}\text { Reduction of TTM symp- } \\
\text { tom severity } \\
\text { NIMH Trichotillomania } \\
\text { Severity Scale (NIMH-TSS) }\end{array}$} & $\begin{array}{l}\text { The mean NIMH- } \\
\text { TSS score for the } \\
\text { TCA with pre- } \\
\text { dominantly SRI } \\
\text { actions interven- } \\
\text { tion group was } \\
\text { 9.3. }\end{array}$ & $\begin{array}{l}\text { The mean reduction of } \\
\text { TTM symptom severi- } \\
\text { ty in the intervention } \\
\text { group was } \\
\mathbf{3} \text { points lower } \\
\text { ( } 6.52 \text { lower to } 0.52 \text { high- } \\
\text { er). }\end{array}$ & & $\begin{array}{l}16 \\
\text { (1 study) }\end{array}$ & $\begin{array}{l}\oplus \ominus \ominus \ominus \\
\text { very low } b, c\end{array}$ & $\begin{array}{l}\text { The evidence is very uncertain } \\
\text { about the effect of TCAs with pre- } \\
\text { dominantly SRI actions on reduc- } \\
\text { tion of TTM symptom severity on } \\
\text { the NIMH-TSS. }\end{array}$ \\
\hline & \multicolumn{2}{|l|}{ Study population } & $\begin{array}{l}\text { RR 4.45 } \\
\text { (0.27 to } 73.81)\end{array}$ & $\begin{array}{l}16 \\
\text { (1 study) }\end{array}$ & $\begin{array}{l}\oplus \ominus \ominus \ominus \\
\text { very low } b, c\end{array}$ & $\begin{array}{l}\text { The evidence is very uncertain } \\
\text { about the effect of TCAs with }\end{array}$ \\
\hline
\end{tabular}


Tolerability of treatment

- dropouts due to ad-

$N=0 / 6 \quad N=3 / 10$

Dropout rate

Follow-up: 9 weeks

*The basis for the assumed risk (e.g. the median control group risk across studies) is provided in footnotes. The corresponding risk (and its $95 \%$ confidence interval) is based on the assumed risk in the comparison group and the relative effect of the intervention (and its $95 \% \mathrm{Cl}$ ).

Cl: confidence interval; RR: risk ratio

GRADE Working Group grades of evidence

High certainty: further research is very unlikely to change our confidence in the estimate of effect.

Moderate certainty: further research is likely to have an important impact on our confidence in the estimate of effect and may change the estimate.

Low certainty: further research is very likely to have an important impact on our confidence in the estimate of effect and is likely to change the estimate.

Very low certainty: we are very uncertain about the estimate.

a Response is defined as the number of participants with TTM who responded to treatment, as assessed by the CGI-I or similar.

bDowngraded by one level due to serious risk of bias. Concerns with attrition bias. Intention-to-treat analysis not used, 3 withdrew from clomipramine group due to adverse events. $40 \%$ overall attrition rate in clomipramine group.

cDowngraded by two levels due to wide confidence interval and very small sample size $(<50)$.

dNumbers presented as raw values for $\mathrm{N}$ (events) in study population (intervention and control groups), and not modelled on RR.

Summary of findings 10. TCAs with predominantly SRI actions versus other TCAs for trichotillomania in adults

\section{TCAs with predominantly SRI actions versus other TCAs for trichotillomania in adults}

Patient or population: adults with trichotillomania

Settings: outpatient

Intervention: TCAs with predominantly SRI actions

Comparison: placebo

\begin{tabular}{|c|c|c|c|c|c|c|}
\hline \multirow[t]{3}{*}{ Outcomes } & \multicolumn{2}{|c|}{ Illustrative comparative risks ${ }^{\star}(95 \% \mathrm{CI})$} & \multirow{3}{*}{$\begin{array}{l}\text { Relative effect } \\
(95 \% \mathrm{CI})\end{array}$} & \multirow{3}{*}{$\begin{array}{l}\text { No of partici- } \\
\text { pants } \\
\text { (studies) }\end{array}$} & \multirow{3}{*}{$\begin{array}{l}\text { Certainty of } \\
\text { the evidence } \\
\text { (GRADE) }\end{array}$} & \multirow[t]{3}{*}{ Comments } \\
\hline & Assumed risk & Corresponding risk & & & & \\
\hline & Control & $\begin{array}{l}\text { TCAs with predominant- } \\
\text { ly SRI actions versus oth- } \\
\text { er TCAs in adults }\end{array}$ & & & & \\
\hline $\begin{array}{l}\text { Treatment respon- } \\
\text { se }^{a}\end{array}$ & $\begin{array}{l}\text { The mean Physi- } \\
\text { cian-rated Clinical } \\
\text { Progress Scale score }\end{array}$ & $\begin{array}{l}\text { The mean treatment re- } \\
\text { sponse in the intervention } \\
\text { group was }\end{array}$ & & $\begin{array}{l}26 \\
\text { (1 study) }\end{array}$ & $\begin{array}{l}\oplus \oplus \ominus \ominus \\
\text { lowb }\end{array}$ & $\begin{array}{l}\text { There was low-certainty evi- } \\
\text { dence for a beneficial effect on } \\
\text { treatment response in the TCAs }\end{array}$ \\
\hline
\end{tabular}


Physician-rated Clinical

Progress Scale

dominantly SRI actions

4 points lower

(6.13 to 1.87 lower).

intervention group

was 4.7 .

Follow-up: 5 weeks
Reduction of TTM

symptom severity

NIMH Trichotilloma-

nia Severity Scale

(NIMH-TSS)

The mean NIMH-TSS

score for the TCA with

predominantly SRI

actions intervention

group was 10.6

\section{The mean reduction of \\ TTM symptom severity in \\ the intervention groups}

was

3.8 points lower

(8.08 lower to 0.48 higher). with predominantly SRI actions group compared to the other

TCAs group on the Physician-rated Clinical Progress Scale.

Follow-up: 5 weeks

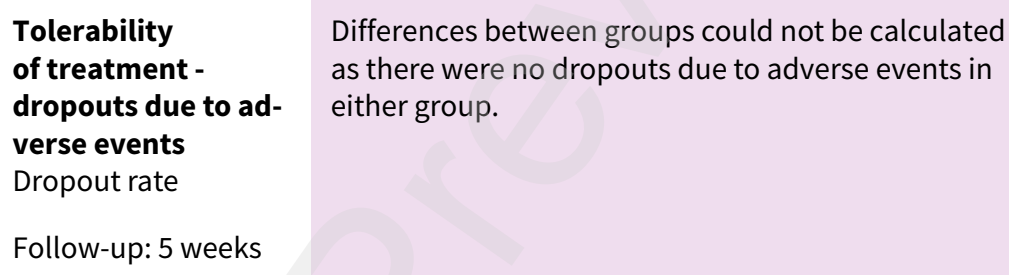

Tolerability

of treatment -

Differences between groups could not be calculated

as there were no dropouts due to adverse events in

dropouts due to ad- either group.

verse events

Dropout rate

Follow-up: 5 weeks

Not estimable

26

(1 study)

lowc

$\oplus \oplus \odot \Theta$

There was low-certainty evidence of no difference in reduction of

TTM symptom severity in the

TCAs with predominantly SRI ac-

tions group compared to the oth-

er TCAs group on the NIMH-TSS.

*The basis for the assumed risk (e.g. the median control group risk across studies) is provided in footnotes. The corresponding risk (and its $95 \%$ confidence interval) is based on the assumed risk in the comparison group and the relative effect of the intervention (and its $95 \% \mathrm{Cl}$ ).

Cl: confidence interval; RR: risk ratio

GRADE Working Group grades of evidence

High certainty: further research is very unlikely to change our confidence in the estimate of effect.

Moderate certainty: further research is likely to have an important impact on our confidence in the estimate of effect and may change the estimate.

Low certainty: further research is very likely to have an important impact on our confidence in the estimate of effect and is likely to change the estimate.

Very low certainty: we are very uncertain about the estimate.

a Response is defined as the number of participants with TTM who responded to treatment, as assessed by the CGI-I or similar.

bDowngraded by two levels due to wide confidence interval and very small sample size $(<50)$.

cDowngraded by two levels due to zero events and very small sample size $(<50)$ 


\section{B A C K G R O U N D}

\section{Description of the condition}

Trichotillomania (TTM) is a disorder characterised by recurrent hair-pulling, resulting in hair loss (APA 2012). It was first named in 1889 by François Henri Hallopeau, a French dermatologist (França 2019). The introduction of TTM into the Diagnostic and Statistical Manual of Mental Disorders, Third Edition, Revised (DSM$I I I-R)$ encouraged treatment studies to proceed. In the DSM-III$R$, TTM was conceptualised as an impulse-control disorder not elsewhere classified. TTM, kleptomania, pyromania and other conditions in the category were characterised by failure to resist an impulse, drive or temptation to perform an act that is harmful to the self or others (APA 1987). TTM remained under Impulse-Control Disorders in DSM-IV.

Based on the diagnostic criteria presented in the Diagnostic and Statistical Manual of Mental Disorders, Fifth Edition (DSM-5) (APA 2012), a diagnosis of TTM requires that recurrent hair-pulling must result in hair loss (criterion A). Evidence of an attempt to decrease or stop hair-pulling must also be present (criterion B). Furthermore, the diagnosis of TTM can be made only if hair-pulling is not better accounted for by another disorder (e.g. in response to a delusion, or as part of a body dysmorphic disorder) and is not a result of a general medical condition (criterion C). Finally, for a diagnosis of TTM, recurrent hair-pulling must cause significant distress or impairment of functioning (criterion D).

The Trichotillomania Impact Project has emphasised the immense distress and impairment associated with chronic hair-pulling (Woods 2006). People with TTM often have low levels of self-esteem, low psychosocial functioning and other comorbid psychiatric disorders, especially mood and anxiety disorders (Grant 2016; Lochner 2019; Stein 2010). Other body-focused repetitive behaviours (BFRBs) are also important comorbidities that may worsen the severity of the primary disorder (Grant 2016). Community prevalence studies indicate that TTM is a common disorder with a point prevalence estimate of $0.5 \%$ to $2.0 \%$ (Grant 2016). In recent years, the prevalence of TTM and other BFRBs has been estimated as significantly higher. In a survey of 82 college students, Odlaug 2010 found a prevalence rate of $3.91 \%$ for TTM, and in a large study of 4335 college students, Houghton 2018 found that $12.27 \%$ of students met criteria for a clinically significant BFRB, including TTM. The onset of TTM is often in childhood (Odlaug 2012); thus, it is important to have effective treatments that have been tested in the young age group.

Some overlap has been noted between TTM and conditions such as obsessive-compulsive disorder (OCD) (Grant 2007). Many people report that hair-pulling is preceded by urges, and that hair-pulling involves ritualistic behaviours redolent of OCD (Swedo 1992). Early work suggested that people with TTM and people with OCD respond to selective serotonin reuptake inhibitors (SSRIs) (Swedo 1989). This and related research led to the inclusion of TTM in a new section of DSM-5, entitled 'Obsessive-Compulsive and Related Disorders' (OCRD) (APA 2012), as well as under OCRDs in the BFRB subcategory in the International Classification of Diseases - 11th revision (ICD-11) (WHO 2018).

Whilst TTM is currently categorised as an OCRD, there are also important differences between TTM and OCD in symptomatology, as well as in treatment response. TTM is not characterised by obsessive thoughts, and greater overlap may be evident with other obsessive-compulsive spectrum disorders, such as skin-picking and tic disorders (Stein 2010). Findings that TTM responds to lowdose antipsychotics and not to SSRIs are redolent of findings on the pharmacotherapy of tic disorders (Eddy 2011). Neuroimaging and treatment studies have strengthened the relationship between TTM and tic disorders (Lamothe 2019). The phenomenology and treatment of TTM may be closer to those of tic disorders than OCD; therefore, it may be helpful to consider TTM as a tic disorder in clinical practice (Lamothe 2019).

Research on the psychobiology of TTM has increased in recent decades; however, specific mechanisms are yet to be established. A review of the neurobiology of TTM shows that researchers have studied numerous regions of interest in relation to TTM across all cerebral lobes, involving white and grey matter, in cortical and subcortical structures. These include the basal ganglia, amygdala, hippocampus, cerebellum and somatosensory cortex (Chamberlain 2009). White matter tracts involved in habit generation and suppression have also been implicated (Grant 2016). In a functional neuroimaging study $(N=10)$ using singlephoton emission computed tomography (SPECT), 12 weeks of treatment with $20 \mathrm{mg}$ of citalopram, titrated to a maximum dose of $60 \mathrm{mg}$, was associated with reduced activation in frontal and striatal regions (Stein 2002).

Several different pharmacotherapy and psychotherapy interventions have been studied in the treatment of TTM. Medications, cognitive-behavioural therapy (CBT) and their combination have been studied in a number of randomised controlled trials (RCTs) and meta-analyses (Bloch 2007; França 2019; Grant 2016; Jafferany 2018; McGuire 2014; Rehm 2015). Metaanalysis of clomipramine and other serotonin reuptake inhibitors (SRIs) in TTM found a significant beneficial treatment effect (Bloch 2007; McGuire 2014). In a more recent meta-analysis, selective serotonin reuptake inhibitors (SSRIs) were shown to have no benefit, whilst clomipramine demonstrated a benefit in reducing TTM symptoms (Farhat 2020). In terms of psychotherapy specifically, habit-reversal therapy (HRT) may have efficacy in the treatment of TTM (Bloch 2007; França 2019; Grant 2016; McGuire 2014). These findings are limited by the small number of individual trials, a lack of trials targeted at children and adolescents, a lack of long-term follow-up to establish durability of treatment and a lack of acknowledgement of comorbidities (Lee 2019). Whilst pharmacotherapy and psychotherapy have both been utilised for the treatment of TTM, this review focuses on medication.

\section{Description of the intervention}

Early studies of treatment of TTM in adults focused on the use of antidepressants such as SSRIs (e.g. citalopram, escitalopram, fluoxetine, fluvoxamine, paroxetine, sertraline; Christenson 1991a; Christenson 1998; Dougherty 2006; Gabriel 2001; Gadde 2007; Iancu 1996; Koran 1992; Mahr 1993; Stanley 1991; Stein 1997; Streichenwein 1995; Winchel 1992), serotoninnoradrenaline reuptake inhibitors (SNRIs; e.g. venlafaxine; Ninan 1998), and tricyclic antidepressants (TCAs) with predominantly serotonin reuptake inhibitor (SRI) actions (e.g. clomipramine; Black 1992; Ninan 2000; Pollard 1991; Swedo 1989). Other traditional psychotropic agents have also been studied, including antipsychotic agents (e.g. haloperidol, olanzapine, quetiapine, risperidone; Epperson 1999; Sentürk 2002; Van Ameringen 1999; Van Ameringen 2010; White 2011; Yasui-Furukori 2011), 
mood stabilisers (e.g. lithium; Sharma 2008), anticonvulsant agents (e.g. gabapentin, lamotrigine, levetiracetam, tiagabine, topiramate; Adewuya 2008; Leombruni 2010; Lochner 2006), opioid antagonists (e.g. naltrexone; De Sousa 2008; Grant 2014), and other antidepressants such as bupropion (Klipstein 2012). More recent studies have investigated a range of different agents from other classes such as cell signal transducers (e.g. inositol; Leppink 2017), cannabinoids (e.g. dronabinol; Grant 2011), and antioxidants (e.g. silymarin; Grant 2015; Grant 2019). In particular, the use of $\mathrm{N}$ acetylcysteine (NAC), a glutamate modulator, has been increasingly examined in recent years in adults and children, with varying results (Bloch 2013; França 2017; Grant 2009; Odlaug 2007; RodriguesBarata 2012; Silva-Netto 2014; Stewart 2003; Taylor 2014).

Few studies examine the effects of medication specifically in children and adolescents under 18 years old (Adewuya 2008; Bloch 2013; De Sousa 2008; Golubchik 2011). To date, NAC is the only drug studied under randomised, placebo-controlled conditions in children and adolescents with TTM (Bloch 2013). Naltrexone has shown promising results in an open-label pilot study in children with TTM (De Sousa 2008). Valproic acid has also shown good results, albeit in a single case study (Adewuya 2008).

\section{How the intervention might work}

Several functional pathways have been hypothesised to be involved in TTM, involving affect regulation, behavioural addiction and cognitive control (Stein 2006). Certain neuroanatomical structures, including areas of the basal ganglia, amygdala, hippocampus, cerebellum and somatosensory cortex, have also been implicated (Chamberlain 2009). These in turn may involve multiple neurotransmitter systems, including the monoaminergic (serotonergic, dopaminergic, noradrenergic), aminergic (glutamatergic, GABAergic) and others (opioid, cannabinoid).

SSRIs, such as fluoxetine and citalopram, are strongly serotonergic and have little effect on dopamine and noradrenaline reuptake. Inhibiting the reuptake of serotonin from the synaptic cleft leads to increased serotonergic neurotransmission, and clinical efficacy is observed at $70 \%$ to $80 \%$ serotonin receptor occupancy (Stahl 2008). TCAs, such as amitriptyline, also exhibit their effect through inhibiting the reuptake of catecholamines, such as noradrenaline and serotonin (Stahl 2008). Within this class, clomipramine exhibits predominantly serotonin reuptake inhibition, whilst other agents, such as desipramine, exhibit predominantly noradrenaline reuptake inhibition. SNRIs, such as venlafaxine, exhibit both serotonin and noradrenaline reuptake inhibition properties, increasing the activity of both of these monoamines in the brain.

In contrast, antipsychotics, such as olanzapine, are antagonists at certain dopamine and serotonin receptors, thereby modulating downstream effects of these monoamines (Stahl 2008). Mood stabilisers (e.g. lithium) and anticonvulsants, such as gabapentin and lamotrigine, invariably decrease glutamatergic neurotransmission or increase GABAergic activity, resulting in a generalised reduction of cerebral activity (Stahl 2008).

Glutamatergic dysfunction in an area of the reward circuitry, the nucleus accumbens, has been implicated in the pathogenesis of OCD (Chakrabarty 2005). This region is thought to play a role in other conditions for which impulse-control and compulsive behaviours are predominant features, including TTM.
The glutamate modulator NAC increases synaptic glutamate concentrations and stimulates inhibitory metabotropic glutamate receptors to restore glutamatergic homeostasis (Baker 2003a). Cannabinoid agonists, such as dronabinol, may also exert their effect by modulating glutamatergic neurotransmission in the basal ganglia and mesolimbic reward system (Grant 2011; Van der Stelt 2005).

Opioid antagonists, such as naltrexone, may be useful in the treatment of substance-use disorders, possibly by inhibiting the sensation of reward associated with the addictive behaviour. It has been proposed that opioid antagonists may also be beneficial in the treatment of other disorders characterised by repetitive behaviours by modulating mesolimbic dopamine (Ikemoto 2010).

Inositol is an intracellular, carbocyclic sugar and a component of the phosphatidyl inositol second messenger system linked to serotonin, dopamine and glutamate metabolism (Camfield 2011). Silymarin is a milk thistle derivative with antioxidant and antiinflammatory properties, which may have a beneficial effect on dopamine functioning (Lu 2010).

Thus, it is possible that medications may target hypothesised mechanisms (e.g. altering the reward system), reducing hairpulling, or may be effective by addressing comorbid depression and OCD. Further work is needed to fully understand the mechanisms underlying TTM and how medication may be effectively utilised.

\section{Why it is important to do this review}

Previous reviews of pharmacological treatment for TTM concluded that no consistent and robust evidence supports the efficacy of any pharmacological agent in the treatment of TTM (Bloch 2007; Grant 2016; McGuire 2014; Rothbart 2013). As a number of additional trials have been published in recent years, an updated systematic review of medication for TTM is timely. A systematic synthesis of treatment data can also serve as the basis for future treatment guidelines, whilst assisting clinicians in effectively managing this disorder and identifying gaps in the current research base.

The authors updated the systematic review of RCTs of the pharmacotherapy of TTM, incorporating additional published trials, adding Summary of Findings tables and quality assessment according to GRADE, which have been added to Cochrane's standards since the initial publication. These were included as per Cochrane guidelines and using Cochrane software (Higgins 2011; Review Manager 2014).

\section{O B JECTIVES}

To assess the effects of medication for TTM in adults, children and adolescents compared with placebo or other medication.

\section{METHODS}

\section{Criteria for considering studies for this review \\ Types of studies}

All published and unpublished RCTs, including studies with multiple treatment groups, cross-over trials and clusterrandomised controlled trials (cluster-RCTs), were considered for inclusion in the review. We excluded pseudo-randomised trials in the interest of minimising bias. 


\section{Types of participants}

\section{Participant characteristics}

We included participants meeting the DSM-III-R (APA 1980) (or later) criteria for TTM in this review. We did not exclude trials consisting of participants who failed to satisfy the criteria of tension preceding the onset of hair-pulling (criterion B) and pleasure, gratification or relief following hair-pulling (criterion $\mathrm{C}$ ), as people without these symptoms may nevertheless present with clinically significant hairpulling (Christenson 1991b; Du Toit 2001).

We applied no restrictions in terms of age, gender or ethnicity.

\section{Comorbidities}

We did not exclude participants with other comorbid psychiatric conditions.

\section{Setting}

We placed no restrictions on study setting.

\section{Subsets of participants}

Trials that included a subset of participants and met study inclusion criteria were also included in the analysis.

\section{Types of interventions}

\section{Experimental interventions}

For conducting searches and considering studies for inclusion in the review, we grouped specific medication interventions according to medication class. We included trials of medications in any of the following classes.

- Antioxidants (e.g. silymarin).

- Anticonvulsants (e.g. gabapentin, lamotrigine, levetiracetam, tiagabine, topiramate).

- Antipsychotics (e.g. haloperidol, olanzapine, quetiapine, risperidone).

- Benzodiazepines (e.g. alprazolam, bromazepam, clonazepam).

- Cell signal transducers (e.g. inositol).

- Glutamate modulators (e.g. N-acetylcysteine (NAC)).

- Monoamine oxidase inhibitors (MAOIs) (e.g. brofaromine, moclobemide, phenelzine).

- Mood stabilisers (e.g. lithium).

- Opioid antagonists (e.g. naltrexone).

- Selective serotonin reuptake inhibitors (SSRIs) (e.g. citalopram, escitalopram, fluoxetine, fluvoxamine, paroxetine, sertraline).

- Serotonin-noradrenaline reuptake inhibitors (SNRIs) (e.g. venlafaxine).

- Tricyclic antidepressants (TCAs) with predominantly SRI actions (e.g. clomipramine).

- Other TCAs (e.g. amitriptyline, desipramine, imipramine).

\section{Control conditions}

Placebo or any agent in the medication classes listed above, or both.

\section{Types of outcome measures}

\section{Primary outcomes}

- Treatment response: response to treatment determined using the Clinical Global Impressions-Improvement item (CGI-I, a widely-used scale ranging from $1=$ 'very much improved' to 7 = 'very much worse'), used as a dichotomous measure of treatment response in which responders are defined as having a change item score of $1=$ 'very much improved' or $2=$ 'much improved' (Guy 1976). Where both dichotomous and continuous data for the CGI-I were presented, we included only the categorical measure of 'responders' versus 'non-responders'. Where CGI-I was not employed, we used other dichotomous measures of treatment response as reported in the original publication. Where no dichotomous measure was available, we report a continuous measure.

- Reduction of TTM symptom severity: reduction in TTM symptom severity based on outcome measures such as the Massachusetts General Hospital Hair-Pulling Scale (MGH-HPS) (Keuthen 1995), or similar, where available. The MGH-HPS is a seven-item, self-report scale that rates urges to pull hair, actual amount of pulling, perceived control over behaviour and distress associated with hair-pulling over the past seven days on a severity scale from 0 to 4 for each item (total scores range from 0 to 28 , with higher scores reflecting greater illness severity). Clinician-rated scales such as the National Institute for Mental Health - Trichotillomania Severity and Impact Scales (NIMH-TSS and NIMH-TIS) are also reported where available. The NIMH-TSS is a six-item assessment, with total scores ranging from 0 to 20 . Higher scores indicate greater severity or impairment. Where multiple scales were used within a study, we report on all for which data are available in the main text and data analysis. For the summary of findings tables, we prioritise reporting MGHHPS as a well-validated scale for use in TTM, although it is a selfreport scale.

\section{Secondary outcomes}

- Reduction of comorbid symptoms of depression: reduction in comorbid symptoms of depression quantified by the widely-used Beck Depression Inventory scale (BDI: Beck 1961), Hamilton Depression Rating scale (HAM-D: Hamilton 1960), or Montgomery-Asberg Depression Rating Scale (MADRS: Montgomery 1979). The Hamilton Depression scale (HAM-D) is a multiple-item questionnaire with 17 to 29 items (depending on the version). People are rated on a 3- or 5-point scale. A score of 0 to 7 is considered to be normal, and a score of 20 or higher, is moderate, severe or very severe. The Beck Depression Inventory (BDI) is a 21-question multiple-choice self-report, one of the most widely-used psychometric tests for measuring the severity of depression. A score of 0 to 9 indicates minimal depression, 10 to 18 mild depression, 19 to 29 moderate depression, and 30 to 63 severe depression. The MADRS is a 10-item diagnostic questionnaire which psychiatrists use to measure the severity of depressive episodes in people with mood disorders. A higher MADRS score indicates more severe depression, and each item yields a score of 0 to 6 . The overall score ranges from 0 to 60 . Usual cutoff points are 0 to 6 - normal/symptom absent; 7 to 19 - mild depression; 20 to 34 - moderate depression; and above 34 - severe depression. Where multiple scales were used within one study, we report only one of them on a hierarchical basis, reporting HAM-D if available, then BDI, MADRS or any other 
measure available. Where no data were available, and this was not an outcome of interest in the primary study, we do not report on this outcome.

- Tolerability of treatment: tolerability of treatment using the following surrogate measures of medication acceptability. Where no data were available, and these were not outcomes of interest in the primary study, we do not report on these outcomes.

- Dropouts due to adverse events: total proportion of people who withdrew from RCTs because of adverse events.

- Common adverse events ( $>20 \%$ ): most common drugrelated adverse events ( $>20 \%$ of participants given the medication affected), as well as significant differences between medication and control groups in the occurrence of drug-related adverse events. We do not report statistics for adverse events affecting less than $20 \%$ of participants but report the rates where available.

- Quality of life - functional disability: quality of life measures, such as the Quality of Life Enjoyment and Satisfaction Questionnaire (Q-LES-Q: Endicott 1993), as well as measures of functional disability, such as the Sheehan Disability Scale (SDS: Sheehan 1996, which includes subscales that assess impairment related to work, social life and family), were included when provided. The Sheehan Disability Scale is a composite of three self-rated items designed to measure the extent to which three major sectors in an individual's life are impaired by panic, anxiety, phobic or depressive symptoms. The person rates the extent to which his or her work, social life or leisure activities, and home life or family responsibilities are impaired by his or her symptoms on a 10-point visual analogue scale. The numerical ratings of 0 to 10 can be translated into a percentage if desired. The three items may be summed into a single dimensional measure of global functional impairment that ranges from 0 (unimpaired) to 30 (highly impaired). The Q-LES-Q is a lengthy, 90-item questionnaire that assesses eight domains of life quality: physical health, subjective feelings, work, household duties, school, leisure activities, social relationships, and general activities. Where multiple scales were used within one study, we report only one of them on a hierarchical basis, reporting SDS if available; otherwise, we report Q-LES-Q or any other available measure. Where no data were available, and this was not an outcome of interest in the primary study, we do not report on this outcome.

\section{Timing of outcome assessment}

When studies reported response rates from baseline at various time points of the trial, treatment indices were subdivided as follows.

- Early response - between one and four weeks; the time point closest to two weeks was given preference.

- Acute phase treatment response - between 6 and 12 weeks; the time point as stated in the original study as the trial endpoint was given preference.

- Follow-up response - between four and six months; the time point closest to 24 weeks was given preference.

The acute phase treatment response - that is between 6 and 12 weeks from baseline - was our primary outcome of interest.

\section{Search methods for identification of studies}

We applied no date or language restrictions to the search for RCTs in any of the sources listed below.

\section{Electronic searches}

An information specialist with the Cochrane Common Mental Disorders (CCMD) group ran searches on the following databases and international trial registers. The search strategies are reported in Appendix 1.

- Cochrane Common Mental Disorders Controlled Trials Register (CCMDCTR) (all available years) (archived database, current to June 2016).

- Cochrane Central Register of Controlled Trials (CENTRAL; 2020, Issue 11) in the Cochrane Library (searched 26 November 2020).

- MEDLINE Ovid (1946 to 26 November 2020).

- Embase Ovid (1974 to 26 November 2020).

- PsycINFO Ovid (from 1806 to 26 November 2020).

- Proquest Dissertations and Thesis Database (PQDT) (all available years, searched 26 November 2020).

- US National Institutes of Health Ongoing Trials Register ClinicalTrials.gov (www.clinicaltrials.gov; searched 26 November 2020).

- World Health Organization International Clinical Trials Registry Platform (apps.who.int/trialsearch; searched 2 December 2019).

We ran additional searches on the following databases, using a single term 'trichotillomania', unless otherwise stated.

- CINAHL EBSCO (Cumulative Index to Nursing and Allied Health Literature; 1982 to 21 January 2021).

- LILACS (Latin American and Caribbean Health Science Information database; 1982 to 21 January 2021) (search: 'trichotillomania' or 'tricotilomania').

- African Index Medicus (all available years, searched 21 January 2021).

- Informit Health Collection (Australia) (1970 to 2020) (searched 21 January 2021).

- Index Medicus for the Eastern Mediterranean Region (1984 to 2020) (searched 21 January 2021).

- IndMed (India) (1985 to 2020) (searched 21 January 2021).

- KoreaMed (1997 to 2020) (searched 21 January 2021).

- Ukraine and the Russian Federation - Panteleimon (1998 to 2020) (searched 21 January 2021).

- Western Pacific Region Index Medicus (all available years, searched 21 January 2021).

We also ran an update of the international trial registers at this time.

- US National Institutes of Health Ongoing Trials Register ClinicalTrials.gov (www.clinicaltrials.gov; searched 21 January 2021);

- World Health Organization International Clinical Trials Registry Platform (apps.who.int/trialsearch; searched 21 January 2021).

Searches conducted for the previous version of the review, Rothbart 2013, are reported in Appendix 2. 


\section{Searching other resources}

\section{Reference lists}

We scanned the bibliographies of all identified trials for additional studies.

\section{Correspondence}

We obtained published and unpublished trials from key researchers, as identified by the frequency with which they are cited in the bibliographies of RCTs and open-label studies.

\section{Grey literature searches}

One review author $(\mathrm{JH})$ performed a search of the following sources of grey literature, theses databases and conference abstracts (all available years to 21 January 2021).

- OpenGrey (previously OpenSIGLE) (greynet.org/).

- Healthcare Management Information Consortium (HMIC; elh.nhs.wales/databases/databases/health-managementinformation-consortium-hmic/) (Ovid)

- National Technical Information Service (www.ntis.gov/).

- Psycextra (www.apa.org/pubs/databases/psycextra).

Theses databases

- Open Access Theses and Dissertations (oatd.org/).

- DART - Europe e-theses portal (www.dart-europe.org/basicsearch.php).

- Networked Digital Library of Theses and Dissertations (www.ndltd.org/).

Conference abstracts

- Abstracts of the American Psychiatric Association (APA) Annual Meeting (www.psychiatry.org/psychiatrists/search-directoriesdatabases/library-and-archive);

- European College of Neuropsychopharmacology Annual Meeting (www.ecnp.eu/; abstracts published in European Neuropsychopharmacology).

We also searched the following pharmaceutical industry trial registers: AstraZeneca; Bristol-Myers Squibb; Eli Lilly; GlaxoSmithKline; Novartis; Roche and Pfizer/Wyeth.

Finally, we conducted a forwards citation search on the Web of Science (reports of included studies) to identify any additional research.

\section{Data collection and analysis}

We used Review Manager 5 (RevMan 5) to perform all analyses reported in this review (Review Manager 2014).

\section{Selection of studies}

Two review authors ( $\mathrm{JH}, \mathrm{TW}$ ) independently examined the titles and abstracts of all studies obtained through the search strategy. The same two review authors obtained and independently assessed the full texts of relevant articles that appeared to meet the inclusion criteria. Each review author then independently applied the full eligibility criteria for inclusion in the review to the full-text articles. Any conflicts of opinion were discussed, and arbitrated by another review author (DJS) if necessary, until consensus was reached.

\section{Data extraction and management}

Two review authors (JH, TW) independently collected data. We resolved any disagreements regarding the data collection procedures through discussion with a third review author (DJS). We created digital data extraction sheets for the purpose of manually recording descriptive information, summary statistics of the outcomes, quality scale ratings and associated commentary. We used Google Forms to set up a data extraction tool to streamline data extraction and entry directly into Excel (example at forms.gle/ zqgpAics3PB6q7ud6). A script was written in Python using the Spyder IDE (version 3.3.6) to aid extraction of trial data housed as XML on ClinicalTrials.gov (www.clinicaltrials.gov). Where available, we compared trial information to published data. Once collated, we entered data into Review Manager 5 (RevMan 5) software, which was used to conduct the meta-analysis. We contacted investigators by email to try to obtain missing information. No reports required translation.

We collected the following information from each included study.

- Description of the trial, including the primary researcher, year of publication, trial protocol identification, number of centres involved, country where the research was conducted and the source of funding.

- Characteristics of the intervention, including the number of participants randomly assigned to the treatment and control groups, the total number of dropouts per group and the number who dropped out because of adverse effects.

- Characteristics of trial methodology, including the recruitment process, diagnostic (e.g. DSM-IV (APA 2000)) and exclusionary criteria employed, the screening instrument used (e.g. the Structured Clinical Interview for DSM-IV (SCID) (Spitzer 1996)) for the primary and comorbid diagnoses, the presence of comorbid major depressive disorder (MDD), the use of a placebo run-in, whether a minimal severity criterion was employed and the length of the study.

- Characteristics of samples, including total number of participants, gender distribution and age distribution, mean age at diagnosis with TTM, mean length of time since diagnosis with TTM, whether participants have been treated with the medication in the past (treatment naivety), the number of participants in the sample with psychiatric comorbidities and the baseline severity of TTM, as assessed by the primary outcome measure reported in the trial or another commonly employed scale.

- Characteristics of the drug, including the drug class, dose, route and frequency of drug administration throughout all trial phases, the duration of the trial and details of any washout period or placebo run-in where applicable.

- Characteristics of the control measure, including type of control utilised and methods for concealing the placebo where applicable.

- Outcome measures employed (primary and secondary) and summary continuous (means and standard deviations) and dichotomous (number of responders) data. We included additional information, such as whether data reflected the intention-to-treat (ITT) with last observation carried forward (LOCF) or completer/observed cases (OC) sample, and the minimal period required for inclusion of participants in the LOCF analyses. We recorded other methods of estimating the outcome 
for participants who dropped out of the study, such as the mixed effects (ME) model.

\section{Main comparisons}

We compared the following medications, grouped by class, against placebo or other active agents, for treating TTM in adults, children and adolescents.

- Antioxidants (e.g. silymarin).

- Antipsychotics (e.g. olanzapine).

- Cell signal transducers (e.g. inositol).

- Glutamate modulators (e.g. N-acetylcysteine).

- Opioid antagonists (e.g. naltrexone).

- SSRIs (e.g. fluoxetine, sertraline).

- TCAs with predominantly SRI actions (e.g. clomipramine).

We planned the following comparisons and grouped specific pharmacological interventions according to medication class based on the included studies.

Comparison 1: antioxidants versus placebo in adults.

Comparison 2: antioxidants versus placebo in children and adolescents.

Comparison 3: antipsychotics versus placebo in adults.

Comparison 4: cell signal transducers versus placebo in adults.

Comparison 5: glutamate modulators versus placebo in adults.

Comparison 6: glutamate modulators versus placebo in children and adolescents.

Comparison 7: opioid antagonists versus placebo in adults.

Comparison 8: SSRIs versus placebo in adults.

Comparison 9: TCAs with predominantly SRI actions versus placebo in adults.

Comparison 10: TCAs with predominantly SRI actions versus other TCAs in adults.

\section{Assessment of risk of bias in included studies}

Two review authors (JH, TW) independently examined the components of each included trial for risk of bias using a standard form. This form included information on sequence generation, allocation concealment, blinding (participants, personnel and outcome assessor), incomplete outcome data, selective outcome reporting and other sources of bias. We assessed the methodological components of the trials as having a low, high or unclear risk of bias, as per the Cochrane Handbook for Systematic Reviews of Interventions (Higgins 2011, hereafter referred to as the Cochrane Handbook). When differences arose, we resolved these through discussion with a third review author (DJS).

\section{Sequence generation}

- Low risk: investigators described a random component in the sequence generation process, such as the use of a random number table, coin tossing, cards or envelope shuffling.
- High risk: investigators described a non-random component in the sequence generation process, such as the use of odd or even date of birth, algorithm based on the day or date of birth, or hospital or clinic record number.

- Unclear: insufficient information was provided to permit judgement of the sequence generation process.

\section{Allocation concealment}

- Low risk: participants and investigators enrolling participants could not foresee assignment (e.g. central allocation or sequentially numbered, opaque, sealed envelopes). Measures were taken to ensure placebo unidentifiable from intervention (e.g. masking of smell of drug and placebo).

- High risk: participants and investigators enrolling participants could foresee upcoming assignment (e.g. open random allocation schedule, such as a list of random numbers, or envelopes that were unsealed or nonopaque or were not sequentially numbered). Placebo was distinct from intervention drug and no attempt was made to conceal this (e.g. clear versus opaque syrup used).

- Unclear: insufficient information was provided to permit judgement of allocation concealment, or the method was not described.

Blinding

- Low risk: blinding of participants, key study personnel and outcome assessors were described, and it is unlikely that blinding could have been broken; or lack of blinding was described in a situation where non-blinding was not likely to introduce bias.

- High risk: no blinding or incomplete blinding was described, and the outcome was likely to be influenced by lack of blinding.

- Unclear: insufficient information was provided to permit judgement of adequacy or otherwise of the blinding.

Incomplete outcome data

- Low risk: no outcome data were missing, reasons for missing outcome data were unlikely to be related to true outcome, or missing outcome data were balanced in number across groups. Intention-to-treat analysis was employed.

- High risk: reason for missing outcome data was likely to be related to the true outcome, with imbalance in numbers across groups or in reasons for missing data. Intention-to-treat analysis was not employed, and no explanation given.

- Unclear: reporting of attrition or exclusions was insufficient.

Selective outcome reporting

- Low risk: a protocol that clearly stated the primary outcome as the same as in the final trial report was available.

- High risk: the primary outcome differs between the protocol and the final trial report.

- Unclear: no trial protocol was available, or reporting was insufficient to determine whether selective reporting was present.

Other sources of bias

- Low risk: no evidence suggested bias from other sources. 
- High risk: potential bias from other sources was present (e.g early stopping of trial, fraudulent activity, extreme baseline imbalance or bias related to specific study design).

- Unclear: insufficient information was provided to permit judgement of adequacy or otherwise of other forms of bias.

\section{Measures of treatment effect}

\section{Dichotomous dato}

We calculated the risk ratio (RR) and the 95\% confidence interval (CI) for the dichotomous outcomes of interest, including treatment response (CGI-I or related measure) and dropouts due to adverse events. We also calculated the number needed to treat for an additional beneficial outcome (NNTB). NNTB is defined as the inverse of the absolute risk difference due to the medication intervention. In this review, we use NNTB to indicate the number of participants who require treatment with medication, relative to a control, before a single additional participant in the medication group responds to treatment. As the outcome measured is favourable, a negative NNT was defined as the NNTB.

\section{Continuous data}

We calculated effect size as mean difference (MD) for continuous summary data where a single outcome scale was used to measure the outcome of interest within a single study or across multiple studies (e.g. MGH-HPS, NIMH-TSS or related measure). For primary outcomes, where multiple scales were used within a single study, we report all as MD. Where multiple scales were employed across multiple studies for a single primary outcome, we planned to calculate the standardised mean difference (SMD). However, this was not required. For secondary outcomes where multiple scales were applied, we selected a single appropriate scale in a hierarchical manner and report the MD (e.g. HAM-D, SDS or related measure)

\section{Skewed data}

When evidence indicated that data were skewed, we planned to obtain individual participant data (when possible) for the purpose of normalising the data through the use of log transformation techniques. If this proved unsuccessful, we planned to exclude those studies that provided skewed data from the analysis. For the purposes of this review, the following constituted evidence of skewness: cases in which the difference between the observed mean and the lowest possible value or the highest possible value on the scale is less than twice as large as the standard deviation (Altman 1996), or cases in which data are reported as skewed by the authors.

\section{Unit of analysis issues}

\section{Studies with multiple treatment groups}

We did not include any studies with multiple treatment groups in this review. In future updates of the review, if trials with three or more arms are identified, it may be appropriate to do a multi-arm trial analysis, wherein the placebo group would be split and shared between two or more groups with smaller sample sizes. In this way, the two or more intervention groups can be analysed as subgroups (Higgins 2011).

In our analyses, for most comparisons, only one study provided data on primary and secondary outcomes. For continuous outcomes, in order to understand the results for each measure used within one trial, and where multiple sclaes were used, these were displayed in one meta-analysis. For these analyses, we have not displayed totals, as these would be misleading.

\section{Cross-over trials}

We included cross-over trials in the calculation of outcomes of interest only: (1) when it was possible to extract medication and placebo or comparator data from the first treatment period; or (2) when inclusion of data from both treatment periods was justified by a washout period of sufficient duration as to minimise the risk of carry-over effects. We defined an adequate washout period in accordance with clinical practice as at least two weeks for all agents, except for fluoxetine, for which a minimum washout period of four weeks was required, given the long plasma half-life of this agent. We included cross-over trials that lacked a washout period if the agents compared were of the same class and had similar properties. For trials in which the washout period was regarded as adequate, we included data from both periods when it was possible to determine the standard error of the mean difference in response between groups (Elbourne 2002). We obtained the summary statistics required to derive the standard error from the trial report. In the future, for trials for which this information is missing, we plan to impute the summary statistics by averaging the relevant statistic from other included cross-over trials with comparable control conditions.

\section{Cluster-randomised trials}

We did not include any cluster-RCTs in this review. In future updates of the review, if cluster-RCTs are identified, we would apply an intraclass correlation (ICC) for the sample to account for the effect of clustering. We would use the ICC reported in the publication, or, if necessary, we would contact study authors to request this information. If we were unable to obtain this information, we would calculate an ICC estimate using the average of the ICCS obtained from the other studies included in the analysis. We would undertake the analysis by using generic inverse variance (GIV).

\section{Dealing with missing data}

We conducted all analyses as ITT. We used the total number of participants randomly assigned to the different comparison groups as the denominator in comparisons of treatment response. Only data from trials that provided information on the original group size (before dropouts) were eligible for inclusion in the analyses of treatment response. We gave preference to the inclusion of summary statistics for continuous outcome measures derived from mixed effects (ME) models, followed by last observation carried forward (LOCF) and observed cases (OC) summary statistics (in that order). This is in line with evidence that ME methods are more robust to bias than LOCF analyses (Verbeke 2000).

\section{Assessment of heterogeneity}

For trials in which the methods and clinical characteristics were sufficiently homogeneous that they could be combined, we assessed statistical heterogeneity of treatment response and reduction of symptom severity visually from the forest plot. This helped us to determine whether differences between the results of trials were greater than would be expected by chance alone. We assessed heterogeneity by using the $\mathrm{Chi}^{2}$ test of heterogeneity. If the $\mathrm{Chi}^{2}$ test had a $\mathrm{P}$ value of less than 0.10 , we interpreted this as 
evidence of heterogeneity, given the low power of the $\mathrm{Chi}^{2}$ statistic when the number of trials is small (Deeks 2021).

In addition, we used the $\mathrm{I}^{2}$ heterogeneity statistic, as reported by RevMan 5, to test the robustness of the $\mathrm{Chi}^{2}$ statistic to differences in the number of trials included in the groups compared within each subgroup analysis (Higgins 2003).

Thresholds for the interpretation of $\mathrm{I}^{2}$ can be misleading because the importance of inconsistency depends on several factors. A rough guide for interpretation was followed:

- $0 \%$ to $40 \%$ : may not be important;

- $30 \%$ to $60 \%$ : may represent moderate heterogeneity;

- $50 \%$ to $90 \%$ : may represent substantial heterogeneity;

- $75 \%$ to $100 \%$ : may represent considerable heterogeneity.

We assessed differences on continuous measures in medication efficacy between these groups by means of Deeks' stratified test of heterogeneity (Deeks 2001). This method subtracts the sum of the $\mathrm{Chi}^{2}$ statistics available for each of the subgroups in the study from the $\mathrm{Chi}^{2}$ statistic available for all of the trials, to obtain a measure $\left(\mathrm{Q}_{b}\right)$ of heterogeneity between groups. We determined differences in treatment response on the CGI-I by noting whether the confidence intervals for the effect sizes of the subgroups overlap. This method was chosen in preference to the stratified test because of inaccuracies in the calculation in RevMan 5 of the $\mathrm{Chi}^{2}$ statistic for dichotomous measures (Deeks 2021).

\section{Assessment of reporting biases}

Publication is not necessarily related to study quality, and indeed publication may imply certain biases (Dickersin 1992; Song 2000). We planned to assess small-sample effects (including publication bias) through visual inspection of a funnel plot of treatment response. This is possible, however, only when more than 10 studies are included in the funnel plot. We did not carry out this assessment in this review due to insufficient studies within comparisons.

\section{Data synthesis}

In conducting a meta-analysis, we obtained categorical and continuous treatment effects from a random-effects model (the random-effects model includes both within-study sampling error and between-studies variation in determining the precision of the confidence interval around the overall effect size, whereas the fixed-effect model takes only within-study variation into account). The outcomes are expressed in terms of an average effect size, as well as by means of $95 \%$ confidence intervals.

In future updates, if appropriate, we may consider using 'multipletreatment meta-analyses' (MTM) methodology to compare and analyse intervention groups across studies. This method relies on the strong assumption that studies of different comparisons are similar in all ways other than the interventions being compared (Higgins 2011).

\section{Subgroup analysis and investigation of heterogeneity}

We planned to undertake subgroup analyses to assess the degree to which methodological differences between trials might have systematically influenced differences observed in primary treatment outcomes. The a priori subgroups that we planned to explore included the following.

- Involvement of participants from a single centre or from multiple centres. Single-centre trials may be associated with lower sample size but less variability in clinician ratings (Youssef 2008).

- Whether trials were industry-funded. In general, published trials that are sponsored by pharmaceutical companies appear more likely to report positive findings than trials that are not supported by for-profit companies (Als-Nielsen 2003; Baker 2003b).

In addition, we planned to use the following criterion to assess the extent of clinical sources of heterogeneity.

- Whether the sample included participants diagnosed with major depressive disorder (MDD). Such an analysis might assist in determining the extent to which the efficacy of a medication used in treating TTM is independent from its ability to reduce symptoms of depression-an important consideration given the classification of many of these medications as antidepressants.

We did not include enough studies to permit subgroup analysis or investigation of the clinical sources of heterogeneity.

\section{Sensitivity analysis}

Sensitivity analyses determine the robustness of the review authors' conclusions to methodological assumptions made in conducting the meta-analysis. There were not enough included studies to permit sensitivity analyses. However, in future updates, we plan to conduct sensitivity analyses to determine whether treatment response varies as a function of:

- the use of treatment response versus non-response as an outcome statistic. This comparison may be necessary in the light of evidence that treatment response may result in less consistent outcome statistics than non-response (Deeks 2002), when the control group event rate is higher than $50 \%$. This sensitivity analysis would accordingly be performed only if most trials report a control group event rate higher than 50\%;

- the exclusion of participants who were lost to follow-up (LTFU). We planned to determine this through a 'worst case/best case' scenario (Deeks 2008). In the worst case, all missing data for the treatment group are recorded as non-responders, whereas in the best case, all missing data for the control group are treated as non-responders. Should conclusions regarding treatment efficacy not differ between these two comparisons, it can be assumed that missing data in trial reports do not have a significant influence on outcome;

- the exclusion of results based on LOCF or OC;

- the exclusion of cross-over trials;

- the exclusion of cluster-randomised trials; or

- varying ICCs in cluster-randomised trials.

\section{Summary of findings and assessment of the certainty of the evidence}

We compiled summary of findings tables to summarise the evidence presented for the outcomes of treatment response, reduction of TTM symptom severity and tolerability of treatment (e.g. dropouts due to adverse events). These outcomes are 
displayed in the summary of findings tables for all comparisons. Where multiple scales were reported for reduction of TTM symptom severity, we report only a single outcome measure for each comparison in our summary of findings tables, prioritising MGHHPS where available, but report on all outcome scales used within the text and data analysis sections of this review. We summarised the findings and graded the certainty of the evidence, in a fixed format using GRADEpro software (GRADEpro GDT).

We classified reasons for downgrading the certainty of the evidence as 'serious' (downgrading the certainty rating by one level) or 'very serious' (downgrading the certainty rating by two levels). We graded the certainty of evidence based on these five factors:

- limitations in the design and implementation of the trial;

- indirectness of evidence;

- unexplained heterogeneity or inconsistency of results;

- imprecision of results;

- high probability of publication bias.

With regards to grading the certainty of the evidence, for risk of bias, we downgraded by one level for every identified source of potential bias. For inconsistency, we downgraded by zero levels if heterogeneity amongst studies was less than 30\%, by one level if heterogeneity was between $30 \%$ and $60 \%$, and by two levels if heterogeneity was greater than $60 \%$. For indirectness, we downgraded based on judgement (four factors). Downgrading for indirectness was based on any concerns related to differences in the study populations, differences in interventions, differences in outcome measures (surrogate outcomes) and indirect comparisons. We did not downgrade due to publication bias as there were insufficient studies to generate funnel plots (Schünemann 2013).

For imprecision, we downgraded by two levels for a 95\% confidence interval that crossed the significance threshold ( 0 for SMD and MD, 1 for RR) and by zero to two levels depending on the sample size.
Sample size thresholds for downgrading were determined using power analysis for 1 study and SMD $=0.3-1$, tested using both fixed-effect and random-effects models. A total of 47 participants were required to detect a change of SMD $=1$ at $80 \%$ power with a random-effects model (low heterogeneity). Using a fixed-effect model, 36 participants are required. For $50 \%$ power, 17 participants are required for fixed-effect and 23 are required for random-effects model analyses. To detect an effect size of 0.3 , a sample size of 469 is required to reach $80 \%$ power using a random-effects model and 353 are needed using a fixed-effect model. The thresholds used for downgrading the certainty of the evidence based on pooled sample size were: 0 levels if more than 400 participants; - 1 level if 50 to 400 participants; and -2 levels if less than 50 participants in the analysis.

We classified the overall certainty in the evidence (or quality of the evidence) for each comparison according to the following categories.

- High certainty: further evidence is unlikely to change our confidence in the estimate of effect.

- Moderate certainty: further research is likely to have an important impact on our confidence in the estimate of effect and may change the estimate.

- Low certainty: further research is very likely to have an important impact on our confidence in the estimate of effect and is likely to change the estimate.

- Very low certainty: we are very uncertain about the estimate.

\section{RES U L T S}

\section{Description of studies}

\section{Results of the search}

Our searches yielded 2458 records. After examination of their titles and abstracts, we obtained the full texts of 22 studies to analyse them for inclusion. Twelve studies met the inclusion criteria for the review (Figure 1). 
Figure 1. PRISMA flow diagram

\begin{tabular}{|l|}
\hline SEARCH \\
PROCESS 1: \\
CCMD Clinical \\
Trial Registry \\
CCMDCTR - \\
references $=60$ \\
refs \\
(Unique records = \\
$60)$
\end{tabular}

\section{SEARCH PROCESS 2: other} databases

CENTRAL $=138$ refs

MEDLINE $=130$ refs

PsycINFO $=142$ refs

Embase $=242$ refs

WHO-ICTRP $=34$ refs

ClinicalTrials.gov $=43$ refs

PQDT (thesis database) $=4$ refs

(Total refs $=733$; Duplicates removed $=403$; Unique records $=$ 330)
SEARCH PROCESS 3: Additional resources

Proquest dissertations and theses $=$ 26 refs; Dissonline $=21$ refs; $\mathrm{CINAHL}$ $=397$ refs; Africa Index Medicus $=0$ refs; Informit Health Collection Australia = 7 refs; Index Medicus for Eastern Mediterranean region $=0$ refs; IndMed = 0 refs; Koreamed = 28 refs; PANTELEIMON (Ukraine $\&$ the Russian Federation) $=0$ refs; Western Pacific Region Index Medicus $=34$ refs; LILACS $=73$ refs;

reference lists $=1$ ref; contact with trialists/authors $=0$ refs; Opensigle $=$ 0 refs; $\mathrm{HMIC}=0$ refs; NTIS = 0 refs; Psycextra $=27$ refs; Pharmaceutical industry trials $=0$ refs; Conferences: $\mathrm{APA}=0$ refs; ECNPP $=0$ refs; OATD $=7$ refs; DART $=1$ ref; NDLTD $=2$ refs; Cited reference search (Web of Science) $=1444$ refs

(Unique records $=2068$ )

SCREENING: no. of records screened after de-duplication

$1=60$

$2=330$

$3=2068$

$\longrightarrow \begin{aligned} & \text { Records } \\ & \text { excluded: } \\ & 2436\end{aligned}$

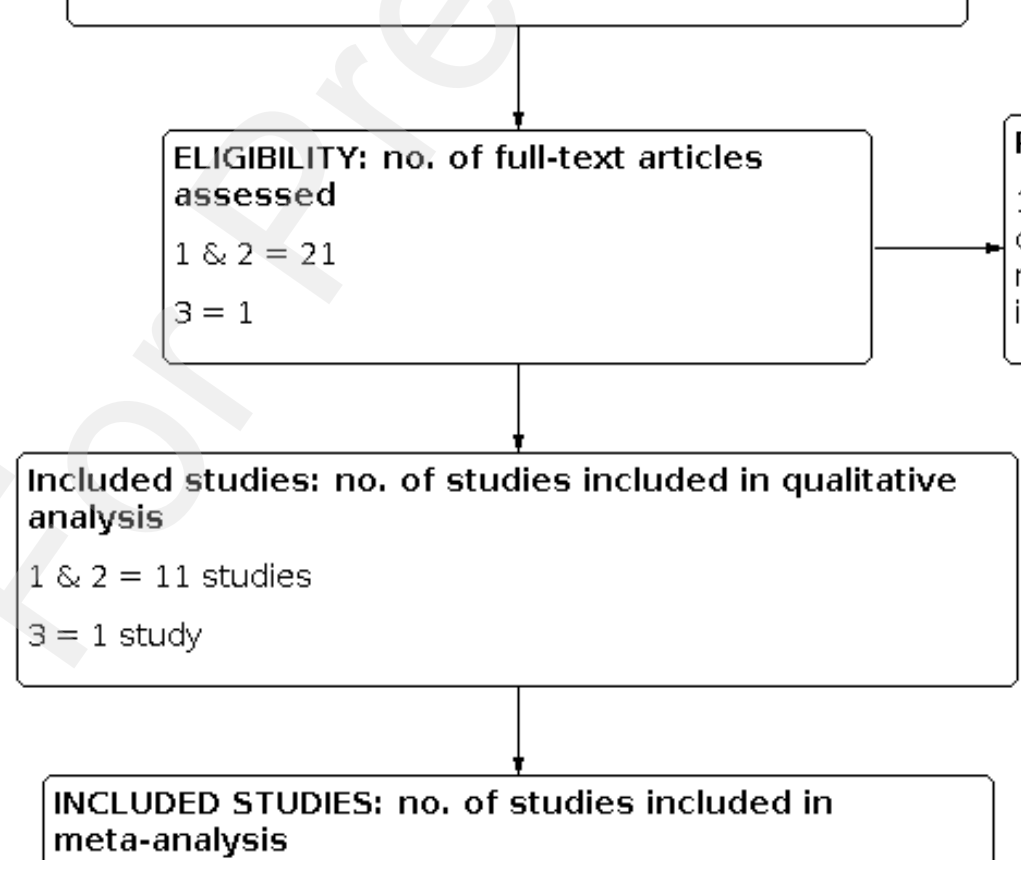


Figure 1. (Continued)

\author{
INCLUDED STUDIES: no. of studies included in \\ meta-analysis \\ $1 \& 2=11$ studies \\ $3=1$ study
}

We attempted to contact the principal investigators of 10 of the 12 included studies to obtain or verify information (eight people, as Christenson authored two studies (Christenson 1991a; Christenson 1994), and Grant was the primary contact person for four studies (Grant 2009; Grant 2014; Grant 2019; Leppink 2017)). We were successful in contacting and obtaining information from six of the eight study authors.

\section{Included studies}

See Characteristics of included studies.

\section{Design}

Of the 12 studies identified as eligible for the review, all were RCTs. Eleven trials were conducted in the United States and one in Canada (Van Ameringen 2010). Only two trials included children and adolescents under 18 years old (Bloch 2013; Grant 2019). Eleven trials were conducted with adult participants aged 18 to 65 . Four of the trials used a cross-over design (Christenson 1991a; Grant 2019; Streichenwein 1995; Swedo 1989), and eight trials used a parallel-group design (Bloch 2013; Christenson 1994; Dougherty 2006; Grant 2009; Grant 2014; Leppink 2017; Ninan 2000; Van Ameringen 2010). The four cross-over trials were 17 weeks, 13 weeks, 27 weeks and 10 weeks in duration, respectively. Of the parallel-group trials, one was 6 weeks in duration, one was 8 weeks in duration, one was 9 weeks in duration, one was 10 weeks in duration, and four were 12 weeks in duration. All of the studies were published in English language journals, with the exception of one (Christenson 1994), which was presented as a conference abstract. Four studies were funded by pharmaceutical companies (Dougherty 2006; Ninan 2000; Streichenwein 1995; Van Ameringen 2010).

\section{Sample sizes}

The eleven studies conducted with adults involved a total of 298 participants with a mean sample size of 27 participants per trial, a median of 24 and a range from the smallest sample size of 13 to the largest of 51 . The single study exclusively in children and adolescents included 39 participants (Bloch 2013). A single study was conducted in adults, children and adolescents, and included 22 participants: 18 adults and 4 children and adolescents (Grant 2019). These adult participants are included in the total reported above.

\section{Setting}

All studies were single-centre trials, and all participants were seen on an outpatient basis.

\section{Participants}

Nine of the 12 trials report using DSM-III-R (or later) criteria to select participants (Bloch 2013; Christenson 1991a; Dougherty 2006; Grant 2009; Grant 2014; Grant 2019; Leppink 2017; Ninan 2000; Van Ameringen 2010). The remaining three trials do not report the specific criteria employed in participant selection (Christenson 1994; Streichenwein 1995; Swedo 1989). Three trials did not require $D S M-I I I-R$ and $-I V$ criteria $B$ and $C$ for inclusion in the trials (i.e. a sense of tension before and a sense of relief after hairpulling) (Bloch 2013; Christenson 1991a; Grant 2009). Of the 10 trials that reported the gender of participants, nine reported the proportion of female participants as $75 \%$ to $100 \%$, reflecting female predominance in TTM (Bloch 2013; Christenson 1991a; Grant 2009; Grant 2014; Grant 2019; Leppink 2017; Ninan 2000; Streichenwein 1995; Swedo 1989). The mean age of adult participants in the eight trials that reported this variable was between 28 and 40 years (Christenson 1991a; Grant 2009; Grant 2014; Leppink 2017; Ninan 2000; Streichenwein 1995; Swedo 1989; Van Ameringen 2010). The average age of participants in the trial on children and adolescents was 13 years (Bloch 2013). The mean age of participants in the trial conducted in children, adolescents and adults was 28.1 years (Grant 2019).

Lifetime prevalence rates of comorbid psychiatric conditions were reported in seven trials in adults. Rates of major depressive disorder or a history of major depressive disorder ranged from $15.8 \%$ to 46.2\% in four trials (Christenson 1991a; Grant 2009; Leppink 2017; Swedo 1989); and rates of anxiety disorders ranged from $20.0 \%$ to $75.0 \%$ in six trials (Christenson 1991a; Grant 2009; Leppink 2017; Streichenwein 1995; Swedo 1989; Van Ameringen 2010). Two studies reported rates of 'another impulse-control disorder' as $20.0 \%$ and $36.0 \%$, respectively (Grant 2009; Van Ameringen 2010). The same two studies reported rates of eating disorders of $4.0 \%$ and $2.0 \%$, respectively. One study reported rates of skin picking disorder as $34.2 \%$, attention-deficit hyperactivity disorder (ADHD) as $2.6 \%$, and obsessive-compulsive disorder (OCD) as 2.6\% (Leppink 2017). One study reported a significant rate of drug and alcohol use among participants (30.8\%; Swedo 1989), and one article stated that $52.9 \%$ of their participants had psychiatric comorbidities, although no further details were available (Grant 2014). The study in children and adolescents reported rates of depression as 33.3\%, anxiety as $23.1 \%$, ADHD as $10 \%$, tic disorder as $7.7 \%$, OCD as $5.1 \%$, and skin picking disorder as $2.6 \%$ (Bloch 2013). The study conducted in children, adolescents and adults reported current psychotropic medicine use as $35 \%$ (adults only $=25 \%$; children and adolescents only $=75 \%$ ), current ADHD as $25 \%$ (adults only $=18.8 \%$; children and adolescents only $=50 \%$ ), current depression as $35 \%$ (adults only $=25 \%$; children and adolescents only $=75 \%$ ), current anxiety as $30 \%$ (adults only $=18.8 \%$; children and adolescents only $=$ $75 \%$ ), current nail-biting as $25 \%$ (adults only $=25 \%$; children and adolescents only $=25 \%$ ) and current skin picking as $60 \%$ (adults only $=50 \%$; children and adolescents only $=100 \%$ ) (Grant 2019).

\section{Interventions}

The 11 studies in adults included three studies of SSRIs (two fluoxetine (Christenson 1991a; Streichenwein 1995), and one sertraline (Dougherty 2006)); two of TCAs with predominantly SRI actions (clomipramine (Ninan 2000; Swedo 1989)); one of 
an antipsychotic (olanzapine (Van Ameringen 2010)); one of a glutamate modulator (NAC (Grant 2009)); two of an opioid antagonist (naltrexone (Christenson 1994; Grant 2014)); one of a carbocyclic sugar involved in cell signal transduction (inositol (Leppink 2017)); and one of a flavonoid derived from milk thistle extract with antioxidant properties (silymarin (Grant 2019)). The single study exclusively in children and adolescents examined a glutamate modulator (NAC (Bloch 2013)). A placebo comparison group was employed in all but one trial, which involved an active agent comparator (Swedo 1989). Of the four trials employing a cross-over design (Christenson 1991a; Grant 2019; Streichenwein 1995; Swedo 1989), two involved a five-week washout period before cross-over between fluoxetine and placebo (Christenson 1991a; Streichenwein 1995). In Swedo 1989, a washout period was not employed, as desipramine was used as an active control and has similar properties to the intervention (clomipramine); therefore, a washout period was not deemed necessary. In Grant 2019, a one-week washout period was employed between intervention (silymarin) and placebo arms. All trials employed gradual dose progression, except for one of the naltrexone trials (Christenson 1994), for which a constant dose was given throughout the trial. All interventions were oral medications or placebo, taken by participants at home.

\section{Outcomes}

In the adult studies, seven trials employed the Clinical Global Impression - Improvement item (CGI-I) Scale to measure treatment response (Dougherty 2006; Grant 2009; Grant 2014; Grant 2019; Leppink 2017; Ninan 2000; Van Ameringen 2010); two trials employed an untitled scale that measured five aspects of selfreported TTM severity (Christenson 1991a; Streichenwein 1995); six trials employed the validated National Institute of Mental Health (NIMH) Trichotillomania Severity Scale (TSS) and the Trichotillomania Impairment Scale (TIS) (Christenson 1994; Grant 2014; Grant 2019; Leppink 2017; Ninan 2000; Swedo 1989); six trials employed the Massachusetts General Hospital Hair-Pulling Scale (MGH-HPS) (Dougherty 2006; Grant 2009; Grant 2014; Grant 2019; Leppink 2017; Van Ameringen 2010); two trials employed the Psychiatric Institute Trichotillomania Scale (PITS) (Dougherty 2006; Grant 2009); one trial employed the Trichotillomania Impact Scale (TTMIS) (Dougherty 2006); and one trial employed the Yale-Brown Obsessive-Compulsive Scale for TTM (Van Ameringen 2010).

Secondary outcome measures employed in adult studies were the Hamilton Rating Scale for Depression (HAM-D) (Christenson 1991a; Dougherty 2006; Grant 2009; Grant 2014; Grant 2019; Leppink 2017; Streichenwein 1995), the Hamilton Rating Scale for Anxiety (HAMA) (Grant 2009; Grant 2014; Grant 2019; Leppink 2017), the Beck Depression Inventory (BDI) (Christenson 1991a; Dougherty 2006; Ninan 2000; Streichenwein 1995; Van Ameringen 2010), the Beck Anxiety Inventory (BAI) (Dougherty 2006; Van Ameringen 2010), the State-Trait Anxiety Inventory (STAI) (Ninan 2000), the NIMH Global Assessment Scales for Depression and Anxiety (NIMH-GASD)
(Swedo 1989), the Quality of Life Enjoyment and Satisfaction Questionnaire (Q-LES-Q) (Dougherty 2006; Van Ameringen 2010), the Quality of Life Inventory (QoLI) (Grant 2009; Grant 2014; Leppink 2017), and the Sheehan Disability Scale (SDS) (Grant 2009; Grant 2014; Grant 2019; Leppink 2017; Van Ameringen 2010).

The studies in children and adolescents used the CGI-I to measure treatment response (Bloch 2013; Grant 2019). In the same studies, the MGH-HPS, the NIMH-TSS and the Trichotillomania Scale for Children-Child and Parent versions (TSC-C,P) were used to measure symptom severity. The Children's Depression Inventory (CDI) was used to measure symptoms of depression in one of the studies (Bloch 2013). HAM-A, HAM-D and SDS were applied in the other study as secondary outcome measures (Grant 2019).

\section{Excluded studies}

See Characteristics of excluded studies.

We excluded 10 studies for the following reasons: non-RCT design (Black 1992; Chamberlain 2010; Epperson 1996; Golubchik 2011; Ninan 1998; Riley 1993; Stein 1995; Stein 1997); waiting-list control group instead of placebo (Van Minnen 2003); and dietary intervention, not pharmacotherapy (Dufour 2010).

\section{Ongoing studies}

We identified four ongoing studies. Three ongoing studies were identified with protocols on ClinicalTrials.gov, and one study on the Iranian Registry of Clinical Trials (en.ICRT.ir).

Of the three identified on ClinicalTrials.gov, one study investigated NAC, glutathione levels and compulsivity in anorexia nervosa and other disorders characterised by compulsive behaviour, including TTM (NCT02794389; completed, no results posted; NCT02794389). The second study is investigating dronabinol, a synthetic cannabinol, in TTM and other body-focused repetitive behaviours (NCT03530800; recruiting; NCT03530800). The last study is focusing on an experimental drug, SXC-2023, which may affect glutamatergic dysfunction and oxidative stress response (NCT03797521; active, recruitment completed; NCT03797521). The study on IRCT.ir investigates the individual and combined effects of habit-reversal therapy (HRT) and citalopram in TTM (IRCT20181009041282N2; active, not recruiting; IRCT20181009041282N2).

\section{Studies awaiting classification}

One study is awaiting publication of phase II trial results, which was conducted under randomised, placebo-controlled conditions (Ninan 1998). However, we could not find a reference for the phase II results of this trial, and therefore have excluded the publication of phase I results under 'Excluded studies'.

\section{Risk of bias in included studies}

Full details of the determination of risk of bias can be found inCharacteristics of included studies and in Figure 2 and Figure 3. 
Figure 2. Risk of bias graph: review authors' judgements about each risk of bias item presented as percentages across all included studies

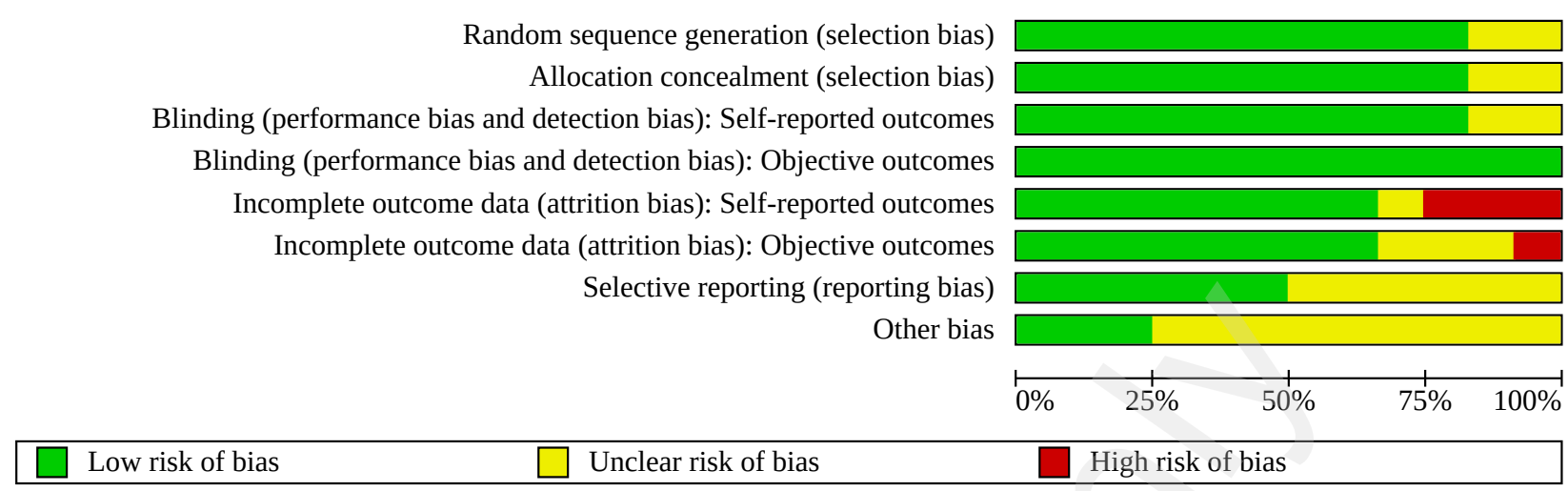


Figure 3. Risk of bias summary: review authors' judgements about each risk of bias item for each included study

\begin{tabular}{|c|c|c|c|c|c|c|c|c|}
\hline & 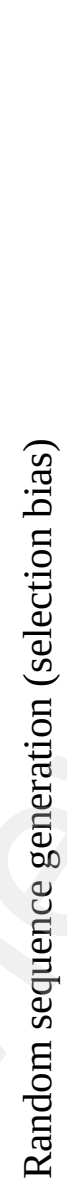 & 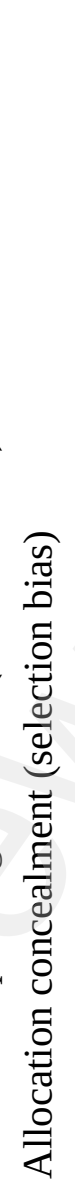 & 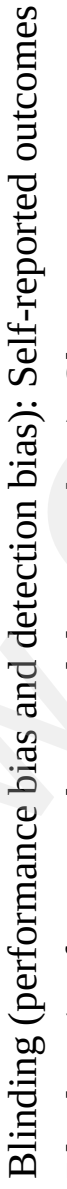 & 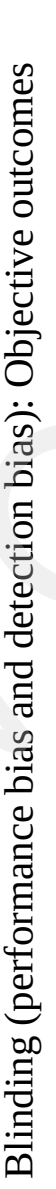 & 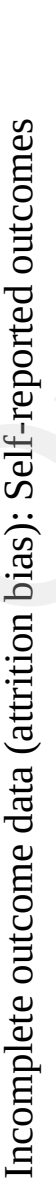 & 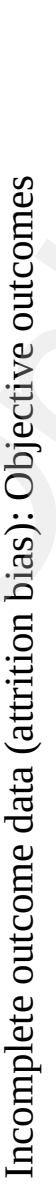 & 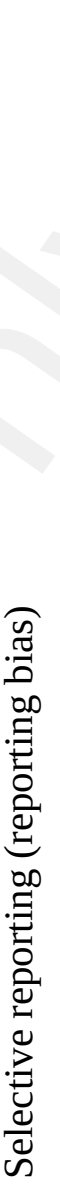 & 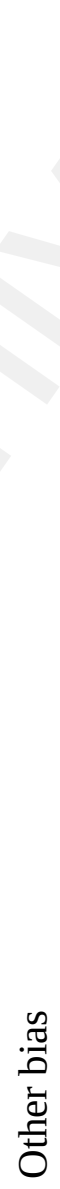 \\
\hline Bloch 2013 & $?$ & + & + & + & + & + & + & $?$ \\
\hline Christenson 1991a & + & + & + & + & + & + & $?$ & + \\
\hline Christenson 1994 & + & + & + & + & $?$ & $?$ & $?$ & + \\
\hline Dougherty 2006 & + & + & + & + & - & - & $?$ & $?$ \\
\hline Grant 2009 & + & + & + & + & + & + & + & $?$ \\
\hline Grant 2014 & + & $?$ & + & + & + & + & + & + \\
\hline Grant 2019 & + & + & + & + & + & + & + & $?$ \\
\hline Leppink 2017 & + & + & + & + & + & + & + & $?$ \\
\hline Ninan 2000 & $?$ & $?$ & $?$ & + & - & $?$ & $?$ & $?$ \\
\hline Streichenwein 1995 & + & + & + & + & - & $?$ & $?$ & $?$ \\
\hline Swedo 1989 & + & + & $?$ & + & + & + & $?$ & $?$ \\
\hline Van Ameringen 2010 & + & + & + & + & + & + & + & $?$ \\
\hline
\end{tabular}




\section{Allocation}

\section{Random sequence generation}

We rated the risk of bias arising from the method of generation of the allocation sequence as low in ten studies (Christenson 1991a; Christenson 1994; Dougherty 2006; Grant 2009; Grant 2014; Grant 2019; Leppink 2017; Streichenwein 1995; Swedo 1989; Van Ameringen 2010). These studies reported generating the sequence by computer or by a random number table, or such information was obtained from the first author. Ninan 2000 and Bloch 2013 did not disclose a method of randomisation; therefore, the presence of selection bias is unclear for these studies.

\section{Allocation concealment}

We rated the risk of bias arising from the method of allocation concealment as low in ten studies (Bloch 2013; Christenson 1991a; Christenson 1994; Dougherty 2006; Grant 2009; Grant 2019; Leppink 2017; Streichenwein 1995; Swedo 1989; Van Ameringen 2010). These studies reported the use of central allocation (e.g. pharmacycontrolled allocation), or this information was obtained from the first author. Ninan 2000 and Grant 2014 did not disclose a method of allocation; therefore, the presence of selection bias is unclear for these studies.

\section{Blinding}

\section{Blinding of participants and personnel (performance bias)}

We rated the risk of bias arising from lack of blinding of participants and personnel as low for 11 of the 12 studies. Most studies reported using a placebo or other form of control that was identical in appearance to the intervention. If not reported, blinding of participants was confirmed by correspondence with the author (Christenson 1991a; Christenson 1994; Dougherty 2006; Grant 2009; Grant 2014; Grant 2019; Leppink 2017; Streichenwein 1995; Swedo 1989; Van Ameringen 2010). Bloch 2013 disclosed quadruple blinding was employed and significant effort was taken to match the placebo to NAC in appearance and smell, thus performance bias was rated as low. Ninan 2000 did not disclose a method for blinding participants, but stated a double-blind study design; therefore, the presence of performance bias is unclear for this study.

\section{Blinding of outcome assessors (detection bias)}

We rated the risk of bias arising from lack of blinding of outcome assessors as low for all studies. Most studies reported blind outcome assessment; if not, this information was obtained from the first author (Bloch 2013; Christenson 1991a; Christenson 1994; Dougherty 2006; Grant 2009; Grant 2014; Grant 2019; Leppink 2017; Ninan 2000; Streichenwein 1995; Swedo 1989; Van Ameringen 2010).

\section{Incomplete outcome data}

We rated risk of attrition bias as low in eight trials which employed ITT analysis or in which attrition rates were not differentially distributed between intervention and control groups, or both (Bloch 2013; Christenson 1991a; Grant 2009; Grant 2014; Grant 2019; Leppink 2017; Swedo 1989; Van Ameringen 2010). We rated the risk of attrition bias as high in three trials, in which attrition was greater than $10 \%$ overall and/or ITT analysis was not employed, and/or attrition rates were differentially distributed between intervention and control groups (Dougherty 2006; Ninan 2000; Streichenwein
1995). Dropout rates were not reported for the Christenson 1994 trial, and therefore we assessed this trial as unclear risk of bias.

\section{Selective reporting}

We obtained trial protocols for six trials, with four trials reported additional outcomes in their publication compared to their protocol (Bloch 2013; Grant 2009; Grant 2014; Leppink 2017). All outcomes from four of the protocols were reported in the publications of the studies. Therefore, we rated the risk of reporting bias for these trials as low (Bloch 2013; Grant 2009; Grant 2014; Leppink 2017). One study reported an outcome in the protocol, but not in the publication (Grant 2019). However, the results would not change the conclusion, and therefore we rated the risk of reporting bias as low (Grant 2019). The other trial protocol obtained revealed no evidence for reporting bias and was also rated as low (Van Ameringen 2010). We did not obtain trial protocols for the remaining six trials, and therefore, we rated the risk of reporting bias as unclear for those trials (Christenson 1991a; Christenson 1994; Dougherty 2006; Ninan 2000; Streichenwein 1995; Swedo 1989).

\section{Other potential sources of bias}

There were few other potential sources of bias. Of note, most studies reported a marked female predominance in percentage of participants, however the effect of this is unclear (Bloch 2013; Christenson 1991a; Dougherty 2006; Grant 2009; Grant 2014; Grant 2019; Leppink 2017; Ninan 2000; Streichenwein 1995; Swedo 1989; Van Ameringen 2010). A few of the studies also reported either industry-funding (Dougherty 2006; Streichenwein 1995; Van Ameringen 2010), medication provided by the pharmaceutical industry (Dougherty 2006; Ninan 2000; Streichenwein 1995), or had authors who worked as consultants for pharmaceutical companies (Grant 2009; Leppink 2017). The relevance of this with regards to potential bias was rated as unclear.

\section{Effects of interventions}

See: Summary of findings 1 Antioxidants versus placebo for trichotillomania in adults; Summary of findings 2 Antioxidants versus placebo for trichotillomania in adolescents; Summary of findings 3 Antipsychotics versus placebo for trichotillomania in adults; Summary of findings 4 Cell signal transducers versus placebo for trichotillomania in adults; Summary of findings 5 Glutamate modulators versus placebo for trichotillomania in adults; Summary of findings 6 Glutamate modulators versus placebo for trichotillomania in children and adolescents; Summary of findings 7 Opioid antagonists versus placebo for trichotillomania in adults; Summary of findings 8 SSRIs versus placebo for trichotillomania in adults; Summary of findings 9 TCAs with predominantly SRI actions versus placebo for trichotillomania in adults; Summary of findings 10 TCAs with predominantly SRI actions versus other TCAs for trichotillomania in adults

See Summary of findings 1; Summary of findings 2; Summary of findings 3; Summary of findings 4; Summary of findings 5; Summary of findings 6; Summary of findings 7; Summary of findings 8; Summary of findings 9; Summary of findings 10 for more information on the quality of the evidence.

Please refer to Types of outcome measures for a description of the scoring systems used in included studies. 
Comparison 1: antioxidants versus placebo for trichotillomania in adults

\section{Primary outcomes}

\section{Treatment response}

The cross-over study by Grant 2019 showed no evidence of benefit for silymarin (milk thistle extract) compared to placebo with regards to treatment response in adult participants after six weeks of treatment. On the Clinical Global Impressions - Improvement scale (CGI-I), 5 of 14 (35.7\%) participants in the silymarin group compared to 4 of $14(28.6 \%)$ participants in the placebo group showed "much" or "very much" improvement. This excludes data from four participants who were lost to follow-up. There was no difference between groups when analysed using a best-case scenario, ITT analysis (risk ratio (RR) 2.25, 95\% confidence interval (Cl) 0.84 to 5.99; 1 study, 36 participants; low-certainty evidence; Analysis 1.1.1). We rated the certainty of the evidence as low due to high imprecision from a small sample size and wide confidence interval.

\section{Reduction of TTM symptom severity}

In this single trial, adult participants in the silymarin group showed no difference in reduction of TTM symptom severity compared to those in the placebo group on any of the outcome scales employed, including the Clinical Global Impressions - Severity scale (CGI-S: mean difference (MD) $-0.50,95 \% \mathrm{Cl}-1.31$ to $0.31 ; 1$ study, 27 participants; low-certainty evidence; Analysis 1.2.1), the Massachusetts General Hospital Hair-Pulling Scale (MGH-HPS: MD $-1.80,95 \% \mathrm{Cl}-6.22$ to 2.62 ; 1 study, 27 participants; low-certainty evidence; Analysis 1.2.2), and the National Institute of Mental Health - Trichotillomania Severity Scale (NIMH-TSS: MD -1.00, 95\% $\mathrm{Cl}-5.00$ to 3.00; 1 study, 27 participants; low-certainty evidence; Analysis 1.2.3). We rated the certainty of the evidence as low due to high imprecision from a small sample size and wide confidence intervals.

\section{Secondary outcomes}

\section{Reduction of comorbid symptoms of depression}

In the adult participants of the Grant 2019 study, there was no evidence to support the treatment of comorbid symptoms of depression with silymarin versus placebo based on the HAM-D scale (MD 0.60, 95\% Cl -1.82 to 3.02; 1 study, 27 participants; lowcertainty evidence; Analysis 1.3), and both groups were under the threshold for clinical depression at baseline and endpoint. We rated the certainty of this evidence as low for high imprecision due to small sample size and a wide confidence interval.

\section{Quality of life - functional disability}

In the Grant 2019 study, quality of life in adults was assessed using the Sheehan Disability Scale (SDS). There was no evidence to support a beneficial effect of silymarin versus placebo for the improvement in functional impairment (MD $-0.60,95 \% \mathrm{Cl}-6.53$ to 5.33; 1 study, 27 participants; low-certainty evidence; Analysis 1.4). We rated the certainty of this evidence as low for high imprecision due to small sample size and a wide confidence interval.

\section{Tolerability of treatment - dropouts due to adverse events}

In the Grant 2019 trial of silymarin versus placebo, no adult participants withdrew from the study as the result of adverse events ( $0 \%$ for silymarin versus $0 \%$ for placebo; 1 study, 18 participants; low-certainty evidence; Analysis 1.5).

\section{Tolerability of treatment - common adverse events ( $>20 \%$ )}

In the Grant 2019 trial, there were five reports of adverse events in the silymarin group; namely, nausea, upset stomach, bloating, insomnia and headache. In addition, there were four reports of nausea, upset stomach, bloating and dry mouth in the placebo group. The adverse effects were all of mild intensity. None of the adverse effects occurred at a rate of $20 \%$ or more. The trial did not report whether these were in adult or adolescent participants.

\section{Comparison 2: antioxidants versus placebo for trichotillomania in adolescents}

\section{Primary outcomes}

\section{Treatment response}

The adolescents in the study by Grant 2019 showed no difference between silymarin and placebo in terms of number of treatment responders using the CGI-I scale, with two of four participants (50\%) in the silymarin group showing response to treatment as compared to one of four $(25 \%)$ in the placebo group, using an ITT analysis (RR $2.00,95 \% \mathrm{Cl} 0.28$ to 14.20 ; 1 study, 8 participants; low-certainty evidence; Analysis 2.1). We rated the certainty of this evidence as low for high imprecision, due to small sample size and wide confidence interval.

\section{Reduction of TTM symptom severity}

Data calculated for adolescents in this trial showed no difference in reduction of TTM symptom severity for the silymarin group compared to those in the placebo group on any of the outcome scales employed, including the CGI-S (MD -1.13, 95\% Cl -2.72 to 0.46 ; 1 study, 6 participants; low-certainty evidence; Analysis 2.2.1), the MGH-HPS (MD $-5.87,95 \% \mathrm{Cl}-17.46$ to $5.72 ; 1$ study, 6 participants; low-certainty evidence; Analysis 2.2.2), the NIMH-TSS (MD -2.94, 95\% Cl-8.14 to 2.26; 1 study, 6 participants; low-certainty evidence; Analysis 2.2.3), the Trichotillomania Scale for Children - Child version (TSC-C: MD 0.00, 95\% Cl -1.25 to $1.25 ; 1$ study, 6 participants; low-certainty evidence; Analysis 2.2.4), and the TSCParent version (TSC-P: MD $-1.20,95 \% \mathrm{Cl}-1.48$ to $-0.92 ; 1$ study, 6 participants; low-certainty evidence; Analysis 2.2.5). We assessed the certainty of the evidence as low due to high imprecision from a small sample size and wide confidence intervals.

\section{Secondary outcomes}

\section{Reduction of comorbid symptoms of depression}

In the adolescents in the Grant 2019 study, there was no evidence to support the treatment of comorbid symptoms of depression with silymarin versus placebo using the HAM-D scale (MD -1.62, $95 \% \mathrm{Cl}-3.98$ to $0.74 ; 1$ study, 7 participants; low-certainty evidence; Analysis 2.3). Both the silymarin and placebo groups were under the threshold for clinical depression at baseline and endpoint. We rated the certainty of this evidence as low for high imprecision due to small sample size and a wide confidence interval.

\section{Quality of life - functional disability}

Grant 2019 used the SDS to assess functional impairment for adolescents. There was no evidence to support a beneficial effect of silymarin versus placebo with regards to functional impairment (MD $-1.30,95 \% \mathrm{Cl}-8.43$ to 5.83 ; 1 study, 6 participants; low-certainty evidence; Analysis 2.4). We rated the certainty of this evidence as low for high imprecision due to small sample size and a wide confidence interval. 


\section{Tolerability of treatment - dropouts due to adverse events}

In the Grant 2019 trial of silymarin versus placebo, no adolescents withdrew from the study as the result of adverse events ( $0 \%$ for silymarin versus $0 \%$ for placebo; 1 study, 4 participants; lowcertainty evidence; Analysis 2.5).

\section{Tolerability of treatment - common adverse events ( $>20 \%$ )}

As reported for adults above, in the single trial comparing silymarin to placebo, there were five reports of adverse effects in the silymarin group (nausea, upset stomach, bloating, insomnia and headache) and four reports of adverse effects in the placebo group (nausea, upset stomach, bloating and dry mouth), none of which occurred at a rate of $20 \%$ or more, and all of mild intensity. The trial did not report whether these were in adult or adolescent participants.

\section{Comparison 3: antipsychotics versus placebo for trichotillomania in adults}

\section{Primary outcomes}

\section{Treatment response}

The study by Van Ameringen 2010 yielded probable moderate treatment effects for olanzapine when compared with placebo on all primary outcome measures and was effective at a mean dose of $10.8 \mathrm{mg}$ per day after a mean duration of 8.2 weeks ( $\mathrm{SD}=3.4$ weeks).

On the CGI-I in a single trial, those in the olanzapine group were more likely to respond to treatment compared with placebo, with 11 of $13(85 \%)$ participants in the olanzapine group showing response to treatment compared to 2 of $12(17 \%)$ in the placebo group, based on low-certainty evidence (RR 5.08, 95\% Cl 1.40 to 18.37; 1 study, 25 participants; low-certainty evidence; Analysis 3.1). The NNT with olanzapine to receive an additional beneficial outcome was 1.5 people ( $95 \% \mathrm{Cl} 1.0$ to 2.5$)$. We rated the certainty of the evidence as low due to high imprecision from a small sample size.

\section{Reduction of TTM symptom severity}

In a single trial, participants in the olanzapine group showed likely greater reduction in TTM symptom severity compared to those in the placebo group, as seen across all outcome scales employed, including the CGI-S (MD -1.68, 95\% Cl -2.31 to $-1.05 ; 1$ study, 25 participants; low-certainty evidence; Analysis 3.2.1), the MGH-HPS (MD $-4.87,95 \% \mathrm{Cl}-8.84$ to $-0.90 ; 1$ study, 25 participants; lowcertainty evidence; Analysis 3.2.2), and the Yale-Brown ObsessiveCompulsive Scale for Trichotillomania (TTM-YBOCS) (MD -7.63, 95\% $\mathrm{Cl}-12.35$ to $-2.91 ; 1$ study, 25 participants; low-certainty evidence; Analysis 3.2.3). We rated the certainty of the evidence as low due to high imprecision from a small sample size. All effect sizes were moderate.

\section{Secondary outcomes}

\section{Tolerability of treatment - dropouts due to adverse events}

In the Van Ameringen 2010 trial of olanzapine versus placebo, no participants withdrew from the study as the result of adverse effects ( $0 \%$ for olanzapine versus $0 \%$ for placebo; 1 study, 25 participants; low-certainty evidence; Analysis 3.3).

\section{Tolerability of treatment - common adverse events ( $>20 \%$ )}

In a single trial, the most common adverse effects in the olanzapine group compared to the placebo group were dry mouth $(54 \%$ for olanzapine $(\mathrm{N}=7)$ versus $0 \%$ for placebo; RR $13.93,95 \% \mathrm{Cl} 0.88$ to $220.37 ; 1$ study, 25 participants; low-certainty evidence; Analysis 3.4.1), fatigue ( $54 \%$ for olanzapine $(\mathrm{N}=7)$ versus $0 \%$ for placebo; $R \mathrm{R}$ $13.93,95 \% \mathrm{Cl} 0.88$ to 220.37 ; 1 study, 25 participants; low-certainty evidence; Analysis 3.4.2), increased appetite (46\% for olanzapine ( $N$ =6) versus $0 \%$ for placebo; RR $12.07,95 \% \mathrm{Cl} 0.75$ to 193.73 ; 1 study, 25 participants; low-certainty evidence; Analysis 3.4.3), headache (38\% for olanzapine $(\mathrm{N}=5)$ versus $33 \%$ for placebo $(\mathrm{N}=4)$; RR $10.21,95 \% \mathrm{Cl} 0.62$ to $167.12 ; 1$ study, 25 participants; low-certainty evidence; Analysis 3.4.4) and weight gain (38\% for olanzapine $(\mathrm{N}=$ 5) versus $8 \%$ for placebo ( $\mathrm{N}=1)$; RR $10.21,95 \% \mathrm{Cl} 0.62$ to $167.12 ; 1$ study, 25 participants; low-certainty evidence; Analysis 3.4.5). Four of the 25 participants experienced no adverse events. We rated the certainty of this evidence as low due to high imprecision from a small sample size and wide confidence intervals.

\section{Reduction of comorbid symptoms of depression}

Treatment of comorbid symptoms of depression was an outcome of interest in the Van Ameringen 2010 study; however, no data were reported. A narrative summary reports little to no effect on the Beck Depression Inventory (Van Ameringen 2010). Summary statistics were not available for further analysis.

\section{Quality of life - functional disability}

Quality of life was an outcome of interest in the Van Ameringen 2010 study; however, no data were reported. A narrative summary reports little to no effect on the SDS and on the Quality of Life Enjoyment and Satisfaction Questionnaire (Q-LES-Q) (Van Ameringen 2010. Summary statistics were not available for further analysis.

\section{Comparison 4: cell signal transducers versus placebo for trichotillomania in adults}

\section{Primary outcomes}

\section{Treatment response}

The study by Leppink 2017 showed no difference between inositol and placebo in terms of number of treatment responders using the CGI-I scale with 8 of $19(42.1 \%)$ in the inositol group showing response to treatment as compared to 6 of $12(50 \%)$ in the placebo group, which excluded 7 participants who failed to complete the study. Using a best-case scenario and ITT analysis, there was no effect of inositol on treatment response (RR 1.33, 95\% Cl 0.57 to 3.11 ; 1 study, 38 participants; low-certainty evidence; Analysis 4.1). We judged the certainty of this evidence as low due to high imprecision, small sample size and wide confidence interval.

\section{Reduction of TTM symptom severity}

Using data from the trial protocol (NCT01875445 on ClinicalTrials.gov) we calculated the effect size of inositol versus placebo and found no difference between inositol and placebo in the reduction of TTM symptom severity on the MGH-HPS (MD $-1.30,95 \% \mathrm{Cl}-5.12$ to 2.52 ; 1 study, 38 participants; low-certainty evidence; Analysis 4.2.1) or on the NIMH-TSS (MD 0.00, 95\% Cl -3.37 to 3.37 ; 1 study, 38 participants; low-certainty evidence; Analysis 4.2.2). We rated the certainty of this evidence as low due to high imprecision, small sample size and wide confidence intervals. 


\section{Secondary outcomes}

\section{Tolerability of treatment - dropouts due to adverse events}

In the Leppink 2017 trial of inositol versus placebo, no participants withdrew from the study as the result of adverse events ( $0 \%$ for inositol versus $0 \%$ for placebo; 1 study, 38 participants; lowcertainty evidence; Analysis 4.3).

\section{Tolerability of treatment - common adverse events ( $>20 \%$ )}

In the Leppink 2017 study, within the inositol treatment group there were 11 minor adverse events reported and one case of ectopic pregnancy, a notable major adverse event. However, it was noted that the participant's current birth control conferred an elevated risk of ectopic pregnancy, thus the event was likely unrelated to inositol.

Other minor adverse experiences reported were of mild intensity and included nausea or upset stomach (4 of $19(21 \%)$ for inositol versus 2 of 19 (10.5\%) for placebo; RR $2.00,95 \% \mathrm{Cl} 0.41$ to 9.65; 1 study, 38 participants; low-certainty evidence; Analysis 4.4.1), stomach pain ( $10.5 \%$ for inositol versus $0 \%$ for placebo), diarrhoea ( $10.5 \%$ for inositol versus $0 \%$ for placebo), gas (5.3\% for inositol versus $0 \%$ for placebo) and headache $(10.5 \%$ for inositol versus $10.5 \%$ for placebo). RR was only calculated for adverse events with an incidence above $20 \%$. There was little to no difference between groups regarding tolerability of treatment. We rated the certainty of this evidence as low due to high imprecision, small sample size and wide confidence interval.

\section{Reduction of comorbid symptoms of depression}

Leppink 2017 measured comorbid depressive symptoms using the Hamilton Depression Rating scale (HAM-D) and found little to no treatment type-by-time interaction for inositol versus placebo (Leppink 2017). No further data were available for analysis.

\section{Quality of life - functional disability}

Leppink 2017 measured quality of life using the Quality of Life Inventory (QoLI) and SDS, and found little to no treatment type-bytime interactions for inositol versus placebo. No further data were available for analysis (Leppink 2017).

\section{Comparison 5: glutamate modulators versus placebo for trichotillomania in adults}

\section{Primary outcomes}

\section{Treatment response}

The study by Grant 2009 of $\mathrm{N}$-acetylcysteine (NAC) versus placebo shows that NAC at a dose of $1200 \mathrm{mg}$ twice a day was probably effective when compared with placebo. This finding was based on all primary outcome measures, although it is based on a single trial. NAC had a probable treatment effect on the CGI-I compared to placebo, with a 3.5 times greater chance of responding to treatment for those receiving NAC (RR $3.50,95 \% \mathrm{Cl} 1.34$ to 9.17 ; 1 study, 50 participants; moderate-certainty evidence; Analysis 5.1), and a number needed to treat (NNT) for an additional favourable outcome of 2.5 people ( $95 \% \mathrm{Cl}$ of 2 to 6 ). We judged the certainty of the evidence as moderate, downgrading for imprecision due to small sample size.

\section{Reduction of TTM symptom severity}

Reduction of symptoms as measured by the CGI-S showed a probable benefit for NAC over placebo, although based on a single trial (MD $-1.24,95 \% \mathrm{Cl}-1.93$ to $-0.55 ; 1$ study, 50 participants; moderate-certainty evidence; Analysis 5.2.1). This likely beneficial effect in favour of NAC over placebo was also found on the patientrated MGH-HPS (MD $-5.60,95 \% \mathrm{Cl}-8.50$ to $-2.70 ; 1$ study, 50 participants; moderate-certainty evidence; Analysis 5.2.2) and the Psychiatric Institute Trichotillomania Scale (PITS: MD -6.90, 95\% $\mathrm{Cl}-10.56$ to -3.24 ; 1 study, 50 participants; moderate-certainty evidence; Analysis 5.2.3). The treatment effect on the MGH-HPS was first noted at nine weeks. We judged the certainty of the evidence as moderate for each scale, and downgraded for imprecision due to small sample size.

\section{Secondary outcomes}

\section{Reduction of comorbid symptoms of depression}

In the Grant 2009 study, which compared NAC to placebo, depression severity was assessed using the HAM-D. No clinical depression was detected in the group at baseline. No evidence was found to support the treatment of comorbid depression with NAC compared to placebo, based on a single trial (HAM-D: MD - $0.96,95 \%$ $\mathrm{Cl}-2.88$ to $0.96 ; 1$ study, 50 participants; low-certainty evidence; Analysis 5.3). We judged the certainty of the evidence as low due to high imprecision from a small sample size and wide confidence interval.

\section{Quality of life - functional disability}

For NAC versus placebo, SDS was used to measure psychosocial functioning and quality of life. Although there appeared to be some improvement in functioning and quality of life for those assigned to the NAC group, the evidence suggests no difference for the outcome on the Sheehan Disability Scale, based on a single trial (SDS: MD $-2.76,95 \% \mathrm{Cl}-5.58$ to 0.06 ; 1 study, 50 participants; low-certainty evidence; Analysis 5.4) (Grant 2009). We rated the certainty of the evidence as low due to high imprecision from a small sample size and wide confidence interval.

\section{Tolerability of treatment - dropouts due to adverse events}

In the Grant 2009 trial of NAC versus placebo, no participants withdrew from the study as the result of adverse events $(0 \%$ for NAC versus $0 \%$ for placebo; 1 study, 50 participants; low-certainty evidence; Analysis 5.5).

\section{Tolerability of treatment - common adverse events ( $20 \%$ )}

In the Grant 2009 trial of NAC versus placebo, participants receiving NAC $(N=25)$ did not experience any adverse effects. Of participants receiving placebo $(\mathrm{N}=25)$, one complained of nausea $(4 \%)$, two of diarrhoea (8\%) and one of cough (4\%). We did not calculate the risk ratio as no adverse event had a prevalence higher than $20 \%$.

\section{Comparison 6: glutamate modulators versus placebo for trichotillomania in children and adolescents}

\section{Primary outcomes}

\section{Treatment response}

The study by Bloch 2013 of $\mathrm{N}$-acetylcysteine (NAC) versus placebo showed little to no treatment effect on the CGI-I for NAC, although based on a single trial in children and adolescents (RR 1.19, 95\% $\mathrm{Cl} 0.37$ to $3.77 ; 1$ study, 39 participants; Analysis 6.1). We rated the 
certainty of this evidence as low due to high imprecision from a small sample size and wide confidence interval.

\section{Reduction of TTM symptom severity}

Evidence for TTM symptom severity reduction, as measured by the MGH-HPS, suggests a potentially large benefit for NAC over placebo in reducing TTM symptom severity, although based on a single trial in children and adolescents (MD $-2.83,95 \% \mathrm{Cl}-3.76$ to -1.90 ; 1 study, 39 participants; low-certainty evidence; Analysis 6.2.1). A likely effect in favour of NAC over placebo was also found on the NIMH-TSS (MD -1.33, 95\% Cl -1.97 to -0.69; 1 study, 39 participants; low-certainty evidence; Analysis 6.2.2). Little to no effect was found for NAC compared to placebo on the Trichotillomania Scale for Children - Child version (TSC-C: MD $-0.08,95 \% \mathrm{Cl}-0.22$ to $0.06 ; 1$ study, 39 participants; low-certainty evidence; Analysis 6.2.3) or Parent version (TSC-P: MD $-0.05,95 \% \mathrm{Cl}-0.16$ to $0.06 ; 1$ study, 39 participants; low-certainty evidence; Analysis 6.2.4). We rated the certainty of this evidence as low due to high imprecision from a small sample size.

\section{Secondary outcomes}

\section{Reduction of comorbid symptoms of depression}

In the Bloch 2013 study, which compared NAC to placebo, depression severity was assessed using the Children's Depression Inventory (CDI). At baseline assessment, $20 \%$ of participants reported current depression. The evidence suggests benefit for the placebo group compared to the NAC group on the CDI, although based on a single trial (MD 3.1, 95\% Cl 1.91 to $4.29 ; 1$ study, 39 participants; low-certainty evidence; Analysis 6.3). We rated the certainty of this evidence as low due to high imprecision from a small sample size.

\section{Tolerability of treatment - dropouts due to adverse events}

In the Bloch 2013 trial of NAC versus placebo, one participant in the NAC group developed a whole-body rash at week 4 and withdrew from the study as a result of this adverse event. There was no difference between NAC and placebo groups regarding attrition rate due to adverse events ( $5 \%$ for NAC versus $0 \%$ for placebo; RR 2.86, $95 \% \mathrm{Cl} 0.12$ to 66.11 ; 1 study, 39 participants; low-certainty evidence; Analysis 6.4). We rated the certainty of this evidence as low due to high imprecision from a small sample size and a wide confidence interval.

\section{Tolerability of treatment - common adverse events ( $>20 \%$ )}

In the Bloch 2013 trial of NAC versus placebo, nausea was experienced by participants receiving both NAC ( 6 of $20 ; 30 \%)$ and placebo (12 of 19; 63\%). Nausea was reported more frequently in the placebo group in this single trial ( $R R 0.47,95 \% \mathrm{Cl} 0.22$ to $1.01 ; 1$ study, 39 participants; low-certainty evidence; Analysis 6.5). There were no other common adverse events $(>20 \%)$ in either group. We rated the certainty of this evidence as low due to high imprecision from a small sample size and a wide confidence interval.

\section{Quality of life - functional disability}

This was not an outcome of interest in the Bloch 2013 study; therefore, no data were available.

\section{Comparison 7: opioid antagonists versus placebo for trichotillomania in adults}

Two studies compared naltrexone to placebo (Christenson 1994; Grant 2014). We report the results of the two studies together, although we could not fully synthesise the results. Most analyses were possible for only one of the two studies (Grant 2014), due to lack of available data from the other study (Christenson 1994). However, we did conduct meta-analysis for the first outcome of treatment response.

\section{Primary outcomes}

\section{Treatment response}

The study by Grant 2014 reports little to no difference between naltrexone and placebo in terms of number of treatment responders on the CGI-I with 9 of 25 (36\%) of those in the naltrexone group showing "much" or "very much" improvement as compared to 9 of $26(34.6 \%)$ of those in the placebo group. The study by Christenson 1994 was not published, and the study authors have not kept the raw data. They report that three (43\%) of the participants receiving naltrexone as compared to zero $(0 \%)$ in the placebo group experienced a greater than $50 \%$ reduction in TTM symptom severity as measured by the NIMH-TSS. Meta-analysis showed no beneficial treatment effect; however, the evidence is very uncertain about the effect of opioid antagonists on treatment response (RR $2.14,95 \% \mathrm{Cl} 0.25$ to $18.17 ; \mathrm{I}^{2}=59 \%$; 2 studies, 68 participants; very low-certainty evidence; Analysis 7.1). We rated the certainty of this evidence as very low due to inconsistency and high imprecision from a small sample size and a wide confidence interval.

\section{Reduction of TTM symptom severity}

In the study by Grant 2014, on the CGI-S, there was no effect of naltrexone versus placebo in reducing TTM symptom severity (MD $-0.37,95 \% \mathrm{Cl}-1.10$ to $0.36 ; 1$ study, 51 participants; low-certainty evidence; Analysis 7.2.1). On the MGH-HPS, their primary outcome for reduction in TTM symptom severity, there was no effect of naltrexone versus placebo (MD $-1.14,95 \% \mathrm{Cl}-4.18$ to $1.90 ; 1$ study, 51 participants; low-certainty evidence; Analysis 7.2.2). Using the NIMH-TSS scale, there was also no effect seen (MD -0.79, 95\% $\mathrm{Cl}-3.62$ to 2.04; 1 study, 51 participants; low-certainty evidence; Analysis 7.2.3). We rated the certainty of the evidence as low due to high imprecision from a small sample size and wide confidence intervals.

Christenson 1994 reported evidence for benefit of naltrexone based on the NIMH-TSS, although summary statistics were unavailable for meta-analysis. The other outcome measures, however, did not yield evidence in favour of naltrexone on the NIMH-Trichotillomania Impairment Scale (NIMH-TIS), the NIMH Physician Rating Scale score, the number of hair-pulling episodes, and the estimated number of hairs pulled as per narrative summaries in the original abstract. We did not assess the certainty of the evidence for this study as we were unable to calculate MD or SMD, and it was not clear which statistical tests were employed.

\section{Secondary outcomes}

\section{Reduction of comorbid symptoms of depression}

In the Grant 2014 study, there was no evidence to support the treatment of comorbid symptoms of depression with naltrexone versus placebo based on the HAM-D scale (MD 0.66, 95\% Cl -1.26 to 
2.58; 1 study, 51 participants; low-certainty evidence; Analysis 7.3), and both groups were under the threshold for clinical depression at baseline and endpoint. We rated the certainty of the evidence as low for high imprecision due to small sample size and a wide confidence interval.

In the Christenson 1994 study, no data measuring comorbid symptoms of depression were available as this was not an outcome of interest.

\section{Quality of life - functional disability}

In the Grant 2014 study, no evidence was found on the SDS to support the use of naltrexone versus placebo for the reduction of functional disability (MD $-1.91,95 \% \mathrm{Cl}-5.82$ to $2.00 ; 1$ study, 51 participants; low-certainty evidence; Analysis 7.4). We rated the certainty of the evidence as low due to high imprecision from a small sample size and a wide confidence interval.

Quality of life - reduction of functional disability was not an outcome of interest for the other naltrexone trial (Christenson 1994); therefore, no data were available.

\section{Tolerability of treatment - common adverse events ( $>20 \%$ )}

In the Grant 2014 study of naltrexone, there were few adverse experiences. Sedation was the only reported adverse effect that occurred more frequently in those taking naltrexone (3 of $25 ; 12 \%$ ) compared to placebo ( 1 of $26 ; 3.8 \%$ ), although based on a single trial (RR 3.12, $95 \%$ Cl 0.35 to 28.03; 1 study, 51 participants; lowcertainty evidence; Analysis 7.5). Liver function testing by alanine aminotransferase (ALT), aspartate aminotransferase (AST) and ALT/ AST ratio demonstrated little to no change between or within groups from baseline to endpoint. We rated the certainty of the evidence as low due to high imprecision from a small sample size and wide confidence interval.

No data related to adverse effects were derived from the other trial on naltrexone versus placebo as this was not an outcome of interest (Christenson 1994).

\section{Tolerability of treatment - dropouts due to adverse events}

This was not an outcome of interest in either the Grant 2014 or Christenson 1994 studies; therefore, no data were available.

\section{Comparison 8: SSRIs versus placebo for trichotillomania in adults}

Within the SSRI class, two studies of fluoxetine versus placebo presented drug-by-period interaction data as $\mathrm{F}$, df and $\mathrm{P}$ values (Christenson 1991a; Streichenwein 1995). Neither of the principal authors of these studies was able to provide the original data from the trials to make individual participant analysis (IDP) possible. Therefore, we describe the results of these trials individually. For the third SSRI study, a sertraline study, we obtained mean change scores for the primary outcome measure from the first author (Dougherty 2006). None of the SSRI trials reported effect of SSRI on treatment response compared to placebo; therefore, data were unavailable for further analysis of this outcome.

\section{Primary outcomes}

\section{Treatment response}

Dichotomous treatment response was not an outcome of interest in the Christenson 1991a, Dougherty 2006 or Streichenwein 1995 studies; therefore, no further data were available for analysis.

\section{Reduction of TTM symptom severity}

Results of the initial 12-week phase of the Dougherty 2006 trial comparing sertraline versus placebo were not published, as this phase of the trial was not the focus of the study. We obtained from the first author the mean change scores on their primary outcome measure, the MGH-HPS (mean change score for sertraline $=-1.53$, SD 5.54; mean change score for placebo $=-1.06$, SD 5.34). Our analysis showed the evidence was very uncertain with regards to the effect of sertraline compared with placebo in reduction of TTM symptom severity, based on a single trial (MD - $0.47,95 \% \mathrm{CI}$ -4.30 to 3.36 ; 1 study, 31 participants; very low-certainty evidence; Analysis 8.2). We judged the certainty of this evidence as very low due to indirectness (using mean change scores rather than MD), high imprecision (wide confidence interval and small sample size) and risk of attrition bias.

\section{Secondary outcomes}

\section{Reduction of comorbid symptoms of depression}

In the cross-over study by Christenson 1991a, mean baseline scores on both the HAM-D and the BDI were below the threshold for clinical depression. Mean differences between fluoxetine and placebo showed no difference on either scale (HAM-D: MD 0.80, 95\% Cl-1.90 to 3.50 ; 1 study, 32 participants; low-certainty evidence; Analysis 8.3). A drug-by-period interaction was noted for the HAM-D scores, but in the opposite direction of that anticipated: an improvement in depression was observed during the placebo phase and a worsening of depression during the fluoxetine phase. We rated the certainty of this evidence as low due to imprecision with a wide confidence interval and small sample size.

In the cross-over study by Streichenwein 1995, mean baseline scores on both the HAM-D and the BDI were below the threshold for clinical depression. Scores from the fluoxetine and placebo treatment periods were combined, so that the effect of fluoxetine cannot be differentiated from the effect of placebo. No drug-byperiod interaction was reported for the HAM-D or the BDI. We did not rate the certainty of this evidence as we were unable to calculate MD or SMD.

No data were available on symptoms of comorbid depression for the pharmacotherapy phase of the trial comparing sertraline versus placebo (Dougherty 2006). However, a narrative summary stated that there were no differences between groups observed on final scores on the HAM-D or BDI (Dougherty 2006).

\section{Tolerability of treatment - dropouts due to adverse events}

In the Christenson 1991a cross-over trial of fluoxetine versus placebo, 1 of 17 participants (5.9\%) withdrew from the trial after developing urticaria during week four of fluoxetine. In the Streichenwein 1995 cross-over trial of fluoxetine versus placebo, 1 of 23 participants (4.3\%) withdrew from the trial during the fluoxetine phase because of a severe serum sickness-like syndrome during week seven. Meta-analysis shows no effect of fluoxetine on dropouts due to adverse events, based on two trials (5.1\% for SSRI 
versus $0 \%$ for placebo; RR $3.00,95 \% \mathrm{Cl} 0.33$ to $27.62 ; I^{2}=0 \%$; 2 studies, 78 participants; low-certainty evidence; Analysis 8.4). We judged the certainty of this evidence as low due to high imprecision from a small sample size and wide confidence interval. No data were available on adverse drug effects for the pharmacotherapy phase of the trial comparing sertraline to placebo, as it was not an outcome of interest for this study (Dougherty 2006).

\section{Tolerability of treatment - common adverse events ( $>20 \%$ )}

In the Christenson 1991a trial, the most common adverse event was nausea, which was reported in 5 of 17 (29.4\%) participants taking fluoxetine and in 2 of 17 (11.8\%) of participants taking placebo (RR 2.5, $95 \% \mathrm{Cl} 0.56$ to $11.16 ; 1$ study, 34 participants; low-certainty evidence; Analysis 8.5.1). We rated the certainty of this evidence as low due to high imprecision and risk of attrition bias. In the Streichenwein 1995 trial, common adverse events were categorised as central nervous system (CNS) and gastrointestinal (GIT) effects. CNS effects (including nightmares, insomnia, dizziness, irritability, anxiety and a feeling of doom) as a category affected 22 of 23 participants $(95.7 \%)$ during the fluoxetine phase of the trial versus 16 of 23 participants $(69.6 \%)$ during the placebo phase in this single trial (RR $1.38,95 \% \mathrm{Cl} 1.04$ to $1.83 ; 1$ study, 46 participants; low-certainty evidence; Analysis 8.5.2). GIT effects (including decreased appetite, diarrhoea, constipation, nausea, increased weight, abdominal pain and dyspepsia) as a category affected 14 of 23 participants $(60.9 \%)$ in the fluoxetine phase of the trial and only 3 of 23 participants (13.0\%) during the placebo phase (RR 4.67, 95\% $\mathrm{Cl} 1.55$ to $14.09 ; 1$ study, 46 participants; low-certainty evidence; Analysis 8.5.3). We rated the certainty of this evidence as low due to high imprecision and risk of attrition bias. No data were available on adverse drug effects for the pharmacotherapy phase of the trial comparing sertraline versus placebo as it was not an outcome of interest for this trial (Dougherty 2006).

\section{Quality of life - functional disability}

There were no data available for analysis of the effect of sertraline on quality of life or functional disability compared to placebo. A narrative summary of the effect of sertraline on quality of life compared to placebo states that no differences between groups were observed in final scores on the Quality of Life Enjoyment and Satisfaction Questionnaire (Q-LES-Q) (Dougherty 2006).

Data were unavailable from the fluoxetine versus placebo trials as quality of life was not an outcome of interest in these studies (Christenson 1991a; Streichenwein 1995).

\section{Comparison 9: TCAs with predominantly SRI actions versus placebo for trichotillomania in adults}

One parallel-arm trial of clomipramine versus placebo is included in the review (Ninan 2000). The evidence was very uncertain regarding the efficacy of clomipramine in this study.

\section{Primary outcomes}

\section{Treatment response}

In the study by Ninan 2000, scores on the CGI-I favoured clomipramine, with 4 of 10 participants in the clomipramine group showing response to treatment, compared with 0 of 6 in the placebo group. The evidence from this single trial was very uncertain (RR $5.73,95 \% \mathrm{Cl} 0.36$ to 90.83 ; 1 study, 16 participants; very lowcertainty evidence; Analysis 9.1). We rated the overall certainty of the evidence as very low due to risk of attrition bias and high imprecision from a small sample size and wide confidence interval.

\section{Reduction of TTM symptom severity}

Reduction of TTM symptom severity was reported on the NIMHTIS and the NIMH-TSS. On the NIMH-TIS, there was very uncertain evidence about the effect of clomipramine on the reduction of TTM symptom severity compared to placebo, based on a single trial (MD $1.60,95 \% \mathrm{Cl}-0.82$ to $4.02 ; 1$ study, 16 participants; very low-certainty evidence; Analysis 9.2.1). We rated the certainty of the evidence as very low due to risk of attrition bias and high imprecision due to small sample size and wide confidence interval.

On the NIMH-TSS, there was very uncertain evidence about the effect of clomipramine on the reduction of TTM symptom severity compared to placebo in a single trial (MD $-3.00,95 \% \mathrm{Cl}-6.52$ to 0.52 ; 1 study, 16 participants; very low-certainty evidence; Analysis 9.2.2). We rated the certainty of the evidence as very low due to risk of attrition bias and high imprecision due to small sample size and wide confidence interval.

\section{Secondary outcomes}

\section{Tolerability of treatment - dropouts due to adverse events}

In the Ninan 2000 trial of clomipramine versus placebo, 3 of 10 participants $(30 \%)$ receiving clomipramine dropped out of the study because of significant drug-related adverse effects, although it is not stated which adverse effects caused participants to withdraw from the study. There was very uncertain evidence for increased dropouts due to adverse events in the clomipramine group compared to the placebo group, based on this single trial (30\% for clomipramine versus $0 \%$ for placebo; RR $4.45,95 \% \mathrm{Cl}$ 0.27 to 73.81 ; 1 study, 16 participants; very low-certainty evidence; Analysis 9.3). We rated the certainty of the evidence as very low due to risk of attrition bias and high imprecision from a small sample size and wide confidence interval.

\section{Tolerability of treatment - common adverse events ( $>20 \%$ )}

Common adverse effects in participants taking clomipramine, in a single trial, were tremor ( 3 of $10 ; 30 \%$ ) (RR $4.45,95 \% \mathrm{Cl} 0.27$ to $73.81 ; 1$ study, 16 participants; very low-certainty evidence; Analysis 9.4.1), sedation ( 2 of $10 ; 20 \%$ ) (RR $3.18,95 \% \mathrm{Cl} 0.18$ to $56.95 ; 1$ study, 16 participants; very low-certainty evidence; Analysis 9.4.2), dry mouth (2 of $10 ; 20 \%$ ) (RR $3.18,95 \% \mathrm{Cl} 0.18$ to $56.95 ; 1$ study, 16 participants; very low-certainty evidence; Analysis 9.4.3) and constipation ( 2 of $10 ; 20 \%$ ) (RR $3.18,95 \% \mathrm{Cl} 0.18$ to $56.95 ; 1$ study, 16 participants; very low-certainty evidence; Analysis 9.4.4). There is very uncertain evidence regarding the effect of clomipramine compared to placebo on the risk of experiencing the above adverse events, although the six participants who were taking placebo in the Ninan 2000 trial reported no adverse events. We rated the certainty of this evidence as very low due to high imprecision from small sample size, wide confidence intervals and risk of attrition bias.

\section{Reduction of comorbid symptoms of depression}

This was not an outcome of interest in the Ninan 2000 study; therefore, no further data were available for analysis. 


\section{Quality of life - functional disability}

This was not an outcome of interest in the Ninan 2000 study; therefore, no further data were available for analysis.

\section{Comparison 10: TCAs with predominantly SRI actions versus other TCAs for trichotillomania in adults}

\section{Primary outcomes}

\section{Treatment response}

The Swedo 1989 study provided evidence that clomipramine may be effective when compared with desipramine on the unvalidated Physician-Rated Clinical Progress scale (PRCP) for treatment response (MD $-4.00,95 \% \mathrm{Cl}-6.13$ to $-1.87 ; 1$ study, 26 participants; low-certainty evidence; Analysis 10.1). We rated the certainty of the evidence as low due to high imprecision from a small sample size. Dichotomous treatment response was not measured using a formal scale in the original study, and therefore dichotomous treatment response is not available for analysis.

\section{Reduction of TTM symptom severity}

Clomipramine may have a beneficial effect on the reduction of TTM symptom severity compared to desipramine on the NIMH-TIS, although based on a single trial (MD $-2.00,95 \% \mathrm{Cl}-3.58$ to $-0.42 ; 1$ study, 26 participants; low-certainty evidence; Analysis 10.2.1). On another measure, the NIMH-TSS, in the same trial clomipramine showed no effect on reduction of TTM symptom severity (MD $-3.80,95 \% \mathrm{Cl}-8.08$ to 0.48 ; 1 study, 26 participants; low-certainty evidence; Analysis 10.2.2). We assessed the overall certainty of this evidence as low due to high imprecision from a small sample size.

\section{Secondary outcomes}

\section{Reduction of comorbid symptoms of depression}

In the study by Swedo 1989, the NIMH-GASD was used to assess severity of depression symptoms. The study authors report that no participants scored in the range of clinical depression at the beginning of the study. There was no difference in effect between clomipramine and desipramine (MD $-0.7,95 \% \mathrm{Cl}-1.6$ to $0.2 ; 1$ study, 26 participants; low-certainty evidence; Analysis 10.3). We judged the overall certainty of this evidence as low due to high imprecision from a small sample size.

\section{Tolerability of treatment - dropouts due to adverse events}

In the Swedo 1989 cross-over study comparing clomipramine versus desipramine, of the 13 participants, none withdrew because of adverse events ( $0 \%$ for clomipramine versus $0 \%$ for desipramine; 1 study, 26 participants; low-certainty evidence; Analysis 10.4).

\section{Tolerability of treatment - common adverse events ( $>20 \%$ )}

The three common adverse events affecting participants in this single trial $(\mathrm{N}=13)$ were constipation (5 of $13(38.5 \%)$ for clomipramine compared with 6 of $13(46.2 \%)$ for desipramine; RR $0.83,95 \% \mathrm{Cl} 0.34$ to 2.06 ; 1 study, 26 participants; lowcertainty evidence; Analysis 10.5.1), dry mouth (5 of $13(38.5 \%)$ for clomipramine and 8 of 13 (61.5\%) for desipramine; RR 0.63 , 95\% $\mathrm{Cl} 0.28$ to $1.41 ; 1$ study, 26 participants; low-certainty evidence; Analysis 10.5 .2 ) and tremor (7 of $13(53.8 \%)$ for clomipramine and 3 of $13(23.1 \%)$ for desipramine; RR $2.33,95 \% \mathrm{Cl} 0.77$ to 7.10; 1 study, 26 participants; low-certainty evidence; Analysis 10.5.3). The authors report that there were no differences between clomipramine and desipramine with regards to adverse effects. We judged the certainty of the evidence for common adverse events as low due to high imprecision from a small sample size and wide confidence intervals.

\section{Quality of life - functional disability}

This was not an outcome of interest in the Swedo 1989 study; therefore, no further data were available for analysis.

\section{Heterogeneity}

There were not enough studies to permit investigation of heterogeneity and its clinical sources. Heterogeneity for individual outcomes is addressed in the relevant section above.

\section{Subgroup analyses}

There were insufficient studies to permit a subgroup analysis $(<10$ studies per subgroup).

\section{Sensitivity analyses}

There were insufficient studies to allow a sensitivity analysis $(<10$ studies for any single outcome).

\section{Publication bias}

There were insufficient studies to permit detection of publication bias ( $<10$ studies in any comparison).

\section{DISCUSSION}

\section{Summary of main results}

Overall, a small body of evidence is available on pharmacotherapy for TTM. Twelve studies, all RCTs, are included in this review, all published between 1989 and 2019. Three studies of SSRIs (Christenson 1991a; Dougherty 2006; Streichenwein 1995), two studies of TCAs with predominantly SRI actions (Ninan 2000; Swedo 1989), two studies of opioid antagonists (Christenson 1994; Grant 2014), and two studies of glutamate modulators were included (Bloch 2013; Grant 2009). The three remaining studies focused on an atypical antipsychotic (Van Ameringen 2010), an antioxidant (Grant 2019), and a cell signal transducer (Leppink 2017). The eleven studies in adults involved a total of 298 participants with a mean sample size of 27 participants per trial and a range of 13 (Swedo 1989), to 51 participants (Grant 2014). Only one included study was conducted exclusively in children and adolescents, which had a sample size of 39 participants (Bloch 2013). One study included 4 adolescent and 18 adult participants in a single crossover study (Grant 2019).

We did not conduct meta-analysis across medication classes due to the varying neurochemical actions of the agents utilised. For adults, meta-analysis within medication class was undertaken for the following select outcomes with sufficient data: treatment response to opioid antagonists across two studies (Christenson 1994; Grant 2014), and dropout rate due to adverse events with SSRIs across two studies (Christenson 1991a; Streichenwein 1995). The remaining classes each contained a single study (antioxidants, antipsychotics, cell signal transducers and glutamate modulators) or had multiple studies that could not be combined due to different comparator groups (TCAs with predominantly SRI actions). We primarily conducted analyses on data from single studies, generating summary statistics in the form of MDs and RRs for our outcomes of interest. For children and adolescents, 
we included only two studies in this review (Bloch 2013; Grant 2019). As they investigated different medications, meta-analysis was not possible. For these studies of a glutamate modulator and an antioxidant, we report summary statistics in the form of MDs and RRs for our outcomes of interest.

Overall, it is difficult to draw high-quality conclusions of treatment efficacy, as most medications are represented by a single, relatively small trial. However, four individual trials in adults demonstrated preliminary evidence for the possible efficacy of three individual agents. Clomipramine (a TCA with predominantly SRI action) showed potential benefit compared to desipramine in reducing TTM symptom severity, with low-certainty (Swedo 1989). Olanzapine, an atypical antipsychotic, also showed a possible beneficial effect on treatment response compared to placebo, and reduced TTM symptom severity, again albeit with low-certainty (Van Ameringen 2010). There was also evidence of a probable, beneficial effect of NAC (a glutamate modulator) on treatment response and reduction of TTM symptom severity in adults, with moderate-certainty in the effect estimate, although based on a single trial (Grant 2009).

A single RCT in children and adolescents found evidence that NAC may have a potential beneficial effect on TTM symptom severity in children and adolescents compared to placebo (Bloch 2013). However, this finding may have been influenced by baseline differences between intervention and placebo groups on the MGHHPS and NIMH-TSS. This finding was also not consistent across all rating scales of TTM symptom severity, suggesting the need for further studies, and certainty in this estimate is low. Moreover, the study showed no beneficial treatment effect of NAC when measuring treatment response on the CGI-I (Bloch 2013). This may indicate differences in the neurobiology of TTM in adults and children. Additionally, the inconsistency between self-reported and clinician-rated scales suggests a need for further scale validation studies in children and adolescents with TTM. As trichotillomania is a disorder commonly occurring in childhood, this warrants further investigation.

The overall certainty of the evidence as per GRADE ratings was low on average, with only NAC having some moderatecertainty evidence for possible beneficial effects in adults. Common factors for downgrading the certainty of evidence included wide confidence intervals and small sample size. We found no further substantial evidence of beneficial or harmful effects for fluoxetine, sertraline, desipramine, naltrexone, silymarin or inositol.

\section{Overall completeness and applicability of evidence}

We have presented data from all published and unpublished trials that assessed the efficacy and tolerability of pharmacotherapeutic options for adult TTM and TTM in children and adolescents. The current evidence base of RCTs is not sufficient to permit robust meta-analysis; thus, there was insufficient evidence to confirm or refute the efficacy or acceptability of any particular agent or class of medication for the treatment of TTM in adults or in children and adolescents. There is a particular lack of randomised, placebocontrolled trials for the treatment of paediatric TTM. As TTM often has its onset in childhood or adolescence, this is an important area that warrants further investigation.

The 12 included studies cover 7 different medication classes; therefore, evidence within individual medication classes is insufficient to generalise from individual agents to class. A greater number of larger studies across a broad range of medication classes is needed before robust conclusions can be drawn from metaanalysis. Nevertheless, evidence from single studies suggests that certain medications (clomipramine, olanzapine, NAC) may provide some benefit in adults (Grant 2009; Swedo 1989; Van Ameringen 2010) or children and adolescents (NAC: Bloch 2013).

Meta-analysis was restricted by methodological heterogeneity in terms of statistical reporting, and the use of a wide variety of TTM symptom scales, including some that have not been well validated or previously published. This is a matter of concern because evidence indicates that unpublished rating scales are more likely to favour the treatment group over the control group (Marshall 2000). Several possible reasons have been proposed for this, one of which is that post hoc 'adjustment' of unpublished scales by removing unfavourable items can skew the results in favour of the treatment group (Marshall 2000). In future studies, the use of one or two validated symptom scales, such as the CGI-I and MGH-HPS, and reporting group means and variance will enhance the meta-analytic options.

It is important for future studies to include participants with psychiatric comorbidities, and to measure and report participant response to medication (e.g. depression and anxiety). The exclusion of participants with comorbid disorders means that study participants are likely not a good representation of clinical populations. Amongst the included studies in this review, three studies excluded participants with comorbid psychiatric disorders (Dougherty 2006; Swedo 1989; Van Ameringen 2010); three studies excluded participants with bipolar disorder, dementia, psychotic disorders or substance use disorders (Grant 2014; Grant 2019; Leppink 2017); one study excluded only participants with bipolar disorder or psychotic disorders (Grant 2009); and two studies did not specify comorbidities (Christenson 1994; Ninan 2000). The study exclusively in children and adolescents excluded participants with bipolar disorder, psychotic disorder, substance use disorder, developmental disorder or mental retardation (Bloch 2013). Where information about comorbidities was available, we extracted and reported it.

With the exception of clomipramine and desipramine, direct comparisons of the relative clinical effectiveness of medications in treating TTM are lacking. Direct comparisons of agents may shed light on the neurobiology and treatment of TTM. Clomipramine, which exhibits potent serotonergic activity, showed potential, positive treatment effects, which were lacking for other strongly serotonergic agents - namely, the SSRIs included in this study. This observation may be explained by clomipramine's noradrenergic metabolite, desmethylclomipramine (Maj 1982). However, desipramine, which has strong noradrenergic effects, failed to produce potentially positive treatment effects. This suggests the involvement of both serotonergic and noradrenergic pathways and is supported by positive findings from an openlabel trial of venlafaxine, a serotonin-noradrenaline reuptake inhibitor (SNRI), which has both serotonergic and noradrenergic effects (Ninan 1998). Thus, the role of specific monoamines requires further study with head-to-head comparisons of agents from within medication classes to investigate the roles of each neurotransmitter in turn.

A multiple-treatment meta-analysis (MTM) might be a useful method in future updates of this review to allow comparison of 
three or more different interventions (potentially from different studies) in one meta-analysis. For MTM to be performed, the included studies would have to be similar in all ways other than the interventions being compared, which is currently not the case.

Given the relative lack of evidence to confirm or refute efficacy of any particular medication in treating TTM in adults or children and adolescents, the presence of a comorbid disorder that is known to be responsive to medication may be useful in choosing a medication. Currently, there is no established firstline recommendation for medication in TTM (Farhat 2020; Grant 2016). The presence of psychiatric comorbidities, such as anxiety or depressive disorders, may play a role in selecting an appropriate treatment option. Similarly, knowledge of common adverse events associated with each medication may further assist clinical decision-making.

Whilst medication may provide some benefit in treating TTM, habitreversal therapy and other psychotherapeutic approaches remain viable first-line treatment options, as discussed in other reviews (Bloch 2007; Farhat 2020; Rehm 2015).

Future treatment options include the cannabinoid agonist dronabinol (NCT03530800 on ClinicalTrials.gov; NCT03530800), an experimental drug targeting System $\mathrm{x}_{\mathrm{C}^{-}}$called SXC-2023 (NCT03797521 on ClinicalTrials.gov; NCT03797521), and other NAC derivatives that improve blood-brain barrier permeability being developed by Promentis Pharmaceuticals (patents.google.com/ patent/WO2015070034A1/en).

\section{Quality of the evidence}

This review included 12 studies of 341 participants, in total spanning 7 different medication classes (SSRIs, TCAs, antipsychotics, opioid antagonists, glutamate modulators, antioxidants and cell signal transducers). The number of participants in the included studies was very small, ranging from 13 to 51 , and 4 of the 12 included studies had fewer than 20 participants. Small sample size was the primary reason for downgrading the certainty of the evidence.

In addition to these small numbers, attrition rates of greater than $10 \%$ were seen in 7 of the 12 trials (Bloch 2013; Dougherty 2006; Grant 2014; Grant 2019; Leppink 2017; Ninan 2000; Streichenwein 1995). In the Dougherty 2006 study, 37 participants were randomly assigned, but only 31 of the 37 participants had their outcome data included in the final analysis, representing an attrition rate of $19 \%$. In the Ninan 2000 study, 23 participants were randomly assigned and data from only 16 participants were analysed, for an attrition rate of 30\%. In the Streichenwein 1995 study, 23 participants were randomly assigned with data from only 16 participants included in the final analysis, for an attrition rate of $30 \%$. These trials did not employ intention-to-treat (ITT) analyses, representing a high risk of attrition bias. Four other studies by Bloch 2013, Grant 2014, Grant 2019 and Leppink 2017 had attrition rates of 10\% (4 of 39 participants), 14\% (7 of 51 participants), 18\% (4 of 22 participants) and $18 \%$ ( 7 of 38 participants), respectively, but all randomised participants were included in analyses using an ITT principle.

The use of different, unvalidated scales in some of the included studies diminishes the certainty of evidence available from these studies. For example, two studies used a self-reported rating scale that relied on participants to count the number of hair-pulling episodes, as well as to rate the urgency and severity of hair-pulling (Christenson 1991a; Streichenwein 1995). Given that hair-pulling is often done without awareness, participants may be limited in their ability to keep track of their own hair-pulling.

Protocols for some of these trials were not publicly available, which limited our ability to determine whether there was reporting bias that might in turn have influenced outcomes. In line with other efforts to enhance replicability, we would encourage publication of TTM clinical trial protocols in the future. More recent trials had published protocols on the ClinicalTrials.gov portal (www.clinicaltrials.gov/), which aids interrogation of possible reporting bias.

Four of the trials included in this review employed a cross-over design (Christenson 1991a; Grant 2019; Streichenwein 1995; Swedo 1989). Failure to include a washout period in the Swedo 1989 trial comparing clomipramine with desipramine may have impacted the trial results because of the persistent effects of these agents on both TTM and comorbid conditions such as depression.

The limited number of studies and the limited number of participants, as well as the high attrition rates in many of the studies and the lack of intention-to-treat analyses, indicate that any conclusions about the use of medication for treating TTM must be made with caution. The NAC and olanzapine trials in adults are methodologically robust (Grant 2009; Van Ameringen 2010). However, the certainty of the evidence (GRADE) from a meta-analytic perspective for treatment response and reduction of TTM symptom severity was moderate for NAC and low for olanzapine (downgraded for high imprecision from small sample sizes). Moderate-certainty evidence implies that further research is likely to have an important impact on our confidence in the estimate of effect and may change the estimate, whereas lowcertainty evidence implies that further research is very likely to have an important impact on our confidence in the estimate of effect and is likely to change the estimate. These studies deserve replication to further examine the efficacy of olanzapine and NAC for TTM in adults.

Over time, we note more consistent reporting of outcomes in both published articles on TTM and protocols on ClinicalTrials.gov. In addition, there have been improvements in study design, use of validated scales and reduction of risk of bias in the included studies, with newer studies reporting outcomes as intention-totreat analyses. More high-quality trials that are appropriately powered are required before a robust meta-analysis can be undertaken. Psychometric evaluation of validated, clinician-rated scales would also aid future synthesis of findings.

\section{Potential biases in the review process}

We attempted to be as comprehensive as possible in the review process. As recommended for all Cochrane reviews, we conducted an extensive and comprehensive search of multiple electronic bibliographic databases to identify trials eligible for inclusion. We applied no language or publication restrictions in our search. Further, we engaged directly with many of the authors to obtain missing data or to ask for clarification when this was required. We minimised potential bias in the conduct of our review by having two independent researchers extract data and assess the methodological quality of each study. This detailed process allowed a thorough assessment of trial conduct and an exploration 
of the possible biases that may be present in each trial. Despite all of the measures taken, it is possible that unpublished data have been reported that we did not find; this represents a potential bias in the review process.

\section{Agreements and disagreements with other studies or reviews}

The methodology employed in this study differs from that employed in the meta-analysis of pharmacological trials conducted by Bloch 2007, Farhat 2020 and McGuire 2014. Unlike in the present review, Bloch 2007, Farhat 2020 and McGuire 2014 chose to perform a meta-analysis of the SSRI trials (Christenson 1991a; Dougherty 2006; Streichenwein 1995), and included a fourth trial, Van Minnen 2003 , which the present review excluded in view of its waiting-list control group (i.e. lack of a placebo group). In addition, Bloch 2007 and Farhat 2020 included trials that used different rating scales. The review authors did not find a statistically significant benefit of SSRIs over placebo (Bloch 2007; Farhat 2020). In the meta-analysis of the clomipramine trials, Bloch 2007 and Farhat 2020 found that clomipramine demonstrated efficacy compared with placebo or active control conditions, and this difference was found to be statistically significant. In the present review, we decided that it was not appropriate to combine the two clomipramine trials in a metaanalysis, as one used a placebo group as a control and the other used desipramine as a control (Ninan 2000; Swedo 1989). McGuire 2014 combined SSRIs and TCAs with predominantly SRI actions in a meta-analysis and found a significant treatment effect (SMD $0.41)$. We chose not to combine these classes in a meta-analysis due to heterogeneity in neurochemical actions. Finally, we report treatment response in terms of a dichotomised measure of the CGII, which is not reported on by Bloch 2007, Farhat 2020 or McGuire 2014.

Compared to our original review, we have included summary of findings tables, applied GRADE criteria to rate the certainty in the evidence presented, included three new studies in adults (Grant 2014; Grant 2019; Leppink 2017), and included two new studies in children and adolescents (Bloch 2013; Grant 2019). Additionally, we calculated the number needed to treat for an additional beneficial outcome (NNTB) for NAC and olanzapine in adults. We also include additional graphs and analyses for all outcomes, including metaanalyses for select outcomes within medication classes and for total effectiveness of medication in TTM.

\section{AUTHORS' CONCLUSIONS}

\section{Implications for practice}

There was insufficient evidence from meta-analysis to confirm or refute efficacy of any particular agent or class of medication in the treatment of trichotillomania (TTM) for any age group. Preliminary evidence has suggested beneficial treatment effects of tricyclic antidepressants (TCAs) with predominantly serotonin reuptake inhibitor (SRI) actions (clomipramine $50 \mathrm{mg}$ titrated up to $250 \mathrm{mg}$ per day; low-certainty evidence), glutamate modulators (N-acetylcysteine (NAC) $1200 \mathrm{mg}$ per day titrated up to 1200 $\mathrm{mg}$ twice daily; moderate-certainty evidence) and antipsychotics (olanzapine $2.5 \mathrm{mg}$ per day titrated up to $10 \mathrm{mg}$ per day; lowcertainty evidence) in adults, based on three individual trials, all of which had relatively small sample sizes (Grant 2009; Swedo 1989; Van Ameringen 2010). There is some low-certainty evidence that NAC reduces TTM symptom severity in children, also based on a single trial (Bloch 2013).

\section{Implications for research}

The existing evidence base of randomised controlled trials includes a mixture of parallel and cross-over trials, participants with a range of comorbid disorders and the use of unpublished rating scales. In addition, the existing trials have relatively small sample sizes, and the body of evidence is of low-certainty because of high attrition rates and lack of intention-to-treat analyses. In the future, trials on a variety of agents, of longer duration, with larger sample sizes and using validated rating instruments (such as the Clinical Global Impressions-Improvement (CGI-I) and the Massachusetts General Hospital Hair-Pulling Scale (MGH-HPS)) are needed. Additionally, inclusion of participants with common comorbidities is encouraged, to better reflect clinical populations. Given the preliminary evidence presented in four small trials of a glutamate modulator, an antipsychotic and a TCA with predominantly SRI actions, further work on these three classes of drugs is needed (Bloch 2013; Grant 2009; Swedo 1989; Van Ameringen 2010). Studies of other classes, such as SNRIs, may be useful in determining differential effects of serotonergic and noradrenergic agents in the treatment of TTM. Whilst preliminary evidence is encouraging, more studies in children and adolescents are required to investigate the effects of NAC seen in these age groups, and to investigate the efficacy of other agents in children.

\section{A C K N O WLEDGEMENTS}

We would like to thank the following principal investigators for the clarification they kindly provided on various aspects of their trials: Gary Christenson, Darin Dougherty, Jon Grant, Suzan Streichenwein, Susan Swedo and Michael Van Ameringen.

We would like to thank Nandi Siegfried for providing teaching and guidance to the principal author of the initial review based on her extensive experience conducting Cochrane Reviews, for acting as arbiter in eligibility and extraction decisions, and for assisting with analysis and writing of the initial review.

The authors and the Cochrane Common Mental Disorders Editorial Team are grateful to the peer reviewers for their time and comments, including Harrison Davies, Sweety Jha and Nuala Livingstone. They would also like to thank Cochrane Copy Edit Support for the team's help.

CRG funding acknowledgement: the National Institute for Health Research (NIHR) is the largest single funding organisation of the CCMD Group.

Disclaimer: the views and opinions expressed herein are those of the review authors and do not necessarily reflect those of the NIHR, the National Health Service or the Department of Health and Social Care. 


\section{RE F E R E N C E S}

\section{References to studies included in this review}

Bloch 2013 \{published data only\}

Bloch M, Panza K, Grant JE, Leckman J, Pittenger C. Nacetylcysteine in the treatment of pediatric trichotillomania: a randomized, double-blind, placebo-controlled trial. Neuropsychopharmacology 2012;38:S270.

* Bloch MH, Panza KE, Grant JE, Pittenger C, Leckman JF. NAcetylcysteine in the treatment of pediatric trichotillomania: a randomized, double-blind, placebo-controlled add-on trial. Journal of the American Academy of Child \& Adolescent Psychiatry 2013;52(3):231-40.

\section{Christenson 1991a \{published data only\}}

* Christenson GA, Mackenzie TB, Mitchell JE, Callies AL. A placebo-controlled, double-blind crossover study of fluoxetine in trichotillomania. American Journal of Psychiatry 1991;148(11):1566-71.

\section{Christenson 1994 \{unpublished data only\}}

* Christenson GA, Crow SJ, Mackenzie TB, Crosby RD, Mitchell JE. A placebo controlled double-blind study of naltrexone for trichotillomania [Abstract NR597]. In: New Research Program and Abstracts. The Sesquicentennial Annual Meeting of the American Psychiatric Association. Vol. 1994. Philadelphia (PA): APA, 1994 May 21-26:212.

\section{Dougherty 2006 \{published data only\}}

* Dougherty DD, Loh R, Jenike MA, Keuthen NJ. Single modality versus dual modality treatment for trichotillomania: sertraline, behavioral therapy, or both? Journal of Clinical Psychiatry 2006;67(7):1086-92.

\section{Grant 2009 \{published data only\}}

* Grant JE, Odlaug BL, Kim SW. N-acetylcysteine, a glutamate modulator, in the treatment of trichotillomania: a doubleblind, placebo-controlled study. Archives of General Psychiatry 2009;66(7):756-63.

\section{Grant 2014 \{published data only\}}

* Grant JE, Odlaug BL, Schreiber LR, Kim SW. The opiate antagonist, naltrexone, in the treatment of trichotillomania: results of a double-blind, placebo-controlled study. Journal of Clinical Psychopharmacology 2014;34(1):134-8.

\section{Grant 2019 \{published data only\}}

* Grant JE, Redden SA, Chamberlain SR. Milk thistle treatment for children and adults with trichotillomania: a double-blind, placebo-controlled, crossover negative study. Journal of Clinical Psychopharmacology 2019;39(2):129-34.

\section{Leppink 2017 \{published data only\}}

* Leppink EW, Redden SA, Grant JE. A double-blind, placebocontrolled study of inositol in trichotillomania. International Clinical Psychopharmacology 2017;32(2):107-14.
Ninan 2000 \{published data only\}

* Ninan PT, Rothbaum BO, Marsteller FA, Knight BT, Eccard MA. A placebo-controlled trial of cognitive-behavioral therapy and clomipramine in trichotillomania. Journal of Clinical Psychiatry 2000;61(1):47-50.

Streichenwein 1995 \{published data only\}

* Streichenwein SM, Thornby JI. A long-term, doubleblind, placebo-controlled crossover trial of the efficacy of fluoxetine for trichotillomania. American Journal of Psychiatry 1995;152(8):1192-6.

Swedo 1989 \{published data only\}

Swedo SE, Lenane MC, Leonard HL. Long-term treatment of trichotillomania (hair pulling). New England Journal of Medicine 1993;329(2):131-2.

* Swedo SE, Leonard HL, Rapoport JL, Lenane MC, Goldberger EL, Cheslow DL. A double-blind comparison of clomipramine and desipramine in the treatment of trichotillomania (hair pulling). New England Journal of Medicine 1989;321(8):497-501.

Swedo SE, Leonard HL. Trichotillomania. An obsessive compulsive spectrum disorder? Psychiatric Clinics of North America 1992;15(4):777-90.

\section{Van Ameringen 2010 \{published data only\}}

* Van Ameringen M, Mancini C, Patterson B, Bennett M, Oakman J. A randomized, double-blind, placebo-controlled trial of olanzapine in the treatment of trichotillomania. Journal of Clinical Psychiatry 2010;71(10):1336-43.

\section{References to studies excluded from this review}

Black 1992 \{published data only\}

* Black DW, Blum N. Trichotillomania treated with clomipramine and a topical steroid. American Journal of Psychiatry 1992;149(6):842-3.

\section{Chamberlain 2010 \{published data only\}}

* Chamberlain SR, Grant JE, Costa A, Muller U, Sahakian BJ. Effects of acute modafinil on cognition in trichotillomania. Psychopharmacology 2010;212(4):597-601.

Dufour 2010 \{published data only\}

* Dufour BD, Adeola O, Cheng HW, Donkin SS, Klein JD, Pajor EA, et al. Nutritional up-regulation of serotonin paradoxically induces compulsive behavior. Nutritional Neuroscience 2010;13(6):256-64.

Epperson 1996 \{published data only\}

* Epperson CN, MCDougle CJ, Price LH. Intranasal oxytocin in trichotillomania. Biological Psychiatry 1996;40(6):559-60.

Golubchik 2011 \{published data only\}

* Golubchik P, Sever J, Weizman A, Zalsman G. Methylphenidate treatment in pediatric patients with attention-deficit/ hyperactivity disorder and comorbid trichotillomania: 
a preliminary report. Clinical Neuropharmacology 2011;34(3):108-10.

\section{Ninan 1998 \{published data only\}}

* Ninan PT, Knight B, Kirk L, Rothbaum BO, Kelsey J, Nemeroff CB. A controlled trial of venlaflaxine in trichotillomania: interim phase I results. Psychopharmacology Bulletin 1998;34(2):221-4.

\section{Riley 1993 \{published data only\}}

* Riley WT, Sood A, Al-Mateen CS. Mixed effects of clomipramine in treating childhood trichotillomania. Journal of Child and Adolescent Psychopharmacology 1993;3(3):169-71.

Stein 1995 \{published data only\}

* Stein DJ, Hollander E, Cohen L, Simeon D, Aronowitz B. Serotonergic responsivity in trichotillomania: neuroendrocrine effects of m-chlorophenylpiperazine. Biological Psychiatry 1995;15(37):414-6.

Stein 1997 \{published data only\}

* Stein DJ, Hollander E, De Carcia C, Cohen L, Simeon D. Behavioural response to $\mathrm{m}$-chlorophenylpiperazine and clonidine in trichotillomania. Journal of Serotonin Research 1997:4(1):11-5.

\section{Van Minnen 2003 \{published data only\}}

* Van Minnen A, Hoogduin KA, Keijsers GP, Hellenbrand I, Hendriks GJ. Treatment of trichotillomania with behavioral therapy or fluoxetine. Archives of General Psychiatry 2003;60:517-22.

\section{References to ongoing studies}

\section{IRCT20181009041282N2 \{published data only\}IRCT20181009041282N2}

IRCT20181009041282N2. Compare the effectiveness of group therapy combination of acceptance and commitment therapy and habit reversal training to drug therapy in reducing symptoms, anxiety and depression of patients with trichotillomania. en.irct.ir/trial/34357 (first received 16 November 2018).

\section{NCT02794389 \{published data only\}}

NCT02794389. Glutathione levels and compulsivity [How do glutathione levels affect compulsivity? A double-blind, placebocontrolled study]. clinicaltrials.gov/ct2/show/NCT02794389 (first received 9 June 2016).

\section{NCT03530800 \{published data only\}}

NCT03530800. Dronabinol in trichotillomania and other body focused repetitive behaviors [A double-blind, placebocontrolled study of dronabinol in trichotillomania and other body focused repetitive behaviors]. clinicaltrials.gov/ct2/show/ NCT03530800 (first received 21 May 2018).

\section{NCT03797521 \{published data only\}}

NCT03797521. A study in patients with trichotillomania (TTM) [A phase 2, double blind, placebo-controlled study to explore the safety, tolerability, and activity of SXC-2023 in adults with moderate to severe trichotillomania (TTM) when dosed for 6 weeks]. clinicaltrials.gov/ct2/show/NCT03797521 (first received 9 January 2019).

\section{Additional references}

\section{Adewuya 2008}

Adewuya EC, Zinser W, Thomas C. Trichotillomania: a case of response to valproic acid. Journal of Child and Adolescent Psychopharmacology 2008;18(5):533-6.

\section{Als-Nielsen 2003}

Als-Nielsen B, Chen W, Gluud C, Kjaergard LL. Association of funding and conclusions in randomized drug trials: a reflection of treatment effect or adverse events? Journal of the American Medical Association 2003;290:921-8.

\section{Altman 1996}

Altman DG, Bland JM. Detecting skewness from summary information. BMJ 1996;313:1200.

\section{APA 1980}

American Psychiatric Association. Diagnostic and Statistical Manual of Mental Disorders (DSM-III). 3rd edition. Washington (DC): American Psychiatric Press, 1980.

\section{APA 1987}

American Psychiatric Association. Diagnostic and Statistica Manual of Mental Disorders (DSM-III-R). 3rd revised edition. Washington (DC): American Psychiatric Association, 1987.

\section{APA 2000}

American Psychiatric Association. Diagnostic and Statistical Manual of Mental Disorders (DSM-IV-TR). 4th text revision edition. Washington (DC): American Psychiatric Association, 2000.

\section{APA 2012}

American Psychiatric Association. Diagnostic and Statistical Manual of Mental Disorders (DSM-V). 5th edition. Washington (DC): American Psychiatric Association, 2012.

\section{Baker 2003a}

Baker DA, McFarland K, Lake RW, Shen H, Toda S, Kalivas PW. $\mathrm{N}$-acetyl cysteine-induced blockade of cocaine-induced reinstatement. Annals of the New York Academy of Sciences 2003;1003(1):349-51.

\section{Baker 2003b}

Baker CB, Johnsrud MT, Crismon ML, Robert AR, Woods SW. Quantitative analysis of sponsorship bias in economic studies of antidepressants. British Journal of Psychiatry 2003;183:498-506.

\section{Beck 1961}

Beck AT, Ward C, Mendelson M. Beck Depression Inventory (BDI). Archives of General Psychiatry 1961;4:561-71.

\section{Bloch 2007}

Bloch MH, Landeros-Weisenberger A, Dombrowski P, Kelmendi B, Wegner R, Nudel J, et al. Systematic review: 
pharmacological and behavioral treatment for trichotillomania. Journal of Biological Psychiatry 2007;62:839-46.

\section{Camfield 2011}

Camfield DA, Sarris J, Berk M. Nutraceuticals in the treatment of obsessive compulsive disorder (OCD): a review of mechanistic and clinical evidence. Progress in Neuro-Psychopharmacology and Biological Psychiatry 2011;35(4):887-95.

\section{Chakrabarty 2005}

Chakrabarty K, Bhattacharyya S, Christopher R, Khanna S. Glutamatergic dysfunction in OCD. Neuropsychopharmacology 2005;30(9):1735-40.

\section{Chamberlain 2009}

Chamberlain SR, Odlaug BL, Boulougouris V, Fineberg NA, Grant JE. Trichotillomania: neurobiology and treatment. Neuroscience \& Biobehavioral Reviews 2009;33(6):831-42.

\section{Christenson 1991b}

Christenson GA, Pyle RL, Mitchell JE. Estimated lifetime prevalence of trichotillomania in college students. Journal of Clinical Psychiatry 1991;52(10):415-7.

\section{Christenson 1998}

Christenson GA, Crow SJ, Mitchell JE, Mackenzie TB, Crosby RD. Fluvoxamine in the treatment of trichotillomania. CNS Spectrums 1998;3(9):64-71.

\section{Cohen 1988}

Cohen J. Statistical power analysis for the behavioral sciences. 2nd edition. Hillsdale (NJ): Lawrence Earlbaum Associates, 1988.

\section{Deeks 2001}

Deeks JJ, Altman DG, Bradburn MJ. Statistical methods for examining heterogeneity and combining results from several studies in meta-analysis. In: Egger M, Davey SG, Altman DG, editors(s). Systematic Reviews in Health Care: Meta-Analysis in Context. 2nd edition. London (UK): BMJ Publication Group, 2001.

\section{Deeks 2002}

Deeks JJ. Issues in the selection of a summary statistic for metaanalysis of clinical trials with binary outcomes. Statistics in Medicine 2002;21:1575-600.

\section{Deeks 2021}

Deeks JJ, Higgins JPT, Altman DG (editors). Chapter 10: Analysing data and undertaking meta-analyses. In: Higgins JPT, Thomas J, Chandler J, Cumpston M, Li T, Page MJ, Welch VA (editors). Cochrane Handbook for Systematic Reviews of Interventions version 6.2 (updated February 2021). Cochrane, 2021. Available from www.training.cochrane.org/handbook..

\section{De Sousa 2008}

De Sousa A. An open-label pilot study of naltrexone in childhood-onset trichotillomania. Journal of Child and Adolescent Psychopharmacology 2008;18(1):30-3.

\section{Dickersin 1992}

Dickersin K, Min YI, Meinert CL. Factors influencing the publication of research results: follow-up of applications submitted to two substantial review boards. Journal of the American Medical Association 1992;267(3):374-8.

\section{Du Toit 2001}

Du Toit PL, Van Kradenburg J, Niehaus DJ, Stein DJ. Characteristics and phenomenology of hair-pulling: an exploration of subtypes. Comprehensive Psychiatry 2001;42(3):247-56

\section{Eddy 2011}

Eddy CM, Rickards HE, Cavanna AE. Treatment strategies for tics in Tourette syndrome. Therapeutic Advances in Neurological Disorders 2011;4(1):25-45.

\section{Elbourne 2002}

Elbourne ER, Altman DG, Higgins JP, Curtin F, Worthington HV, Vail A. Meta-analyses involving cross-over trials: methodological issues. International Journal of Epidemiology 2002;31:140-9.

\section{Endicott 1993}

Endicott J, Nee J, Harrison W, Blumenthal R. Quality of Life Enjoyment and Satisfaction Questionnaire: a new measure. Psychopharmacology Bulletin 1993;29(2):321-6.

\section{Epperson 1999}

Epperson CN, Fasula D, Wasylink S, Price LH, McDougle CJ. Risperidone addition in serotonin reuptake inhibitor-resistant trichotillomania: three cases. Journal of Child and Adolescent Psychopharmacology 1999;9(1):43-9.

\section{Farhat 2020}

Farhat LC, Olfson E, Nasir M, Levine JL, Li F, Miguel EC, et al. Pharmacological and behavioral treatment for trichotillomania: an updated systematic review with meta-analysis. Depression and Anxiety 2020;37(8):715-27.

\section{França 2017}

França K, Lotti T. N-acetyl cysteine in the treatment of trichotillomania. Dermatologic Therapy 2017;30(3):e12446.

\section{França 2019}

França K, Kumar A, Castillo D, Jafferany M, Hyczy da Costa Neto M, Damevska K, et al. Trichotillomania (hair pulling disorder): clinical characteristics, psychosocial aspects, treatment approaches, and ethical considerations. Dermatologic Therapy 2019;32(4):e12622.

\section{Gabriel 2001}

Gabriel A. A case of resistant trichotillomania treated with risperidone-augmented fluvoxamine. Canadian Journal of Psychiatry 2001 ;46(3):285-6.

\section{Gadde 2007}

Gadde KM, Wagner RH 2nd, Connor KM, Foust MS. Escitalopram treatment of trichotillomania. International Clinical Psychopharmacology 2007;22(1):39-42. 


\section{GRADEpro GDT [Computer program]}

McMaster University (developed by Evidence Prime) GRADEpro GDT. Version accessed prior to 8 September 2021. Hamilton (ON): McMaster University (developed by Evidence Prime). Available at gradepro.org.

\section{Grant 2007}

Grant JE, Odlaug BL, Potenza, MN. Addicted to hair pulling? How an alternate model of trichotillomania may improve treatment outcome. Harvard Review of Psychiatry 2007;15:80-5.

\section{Grant 2011}

Grant JE, Odlaug BL, Chamberlain SR, Kim SW. Dronabinol, a cannabinoid agonist, reduces hair pulling in trichotillomania: a pilot study. Psychopharmacology 2011;218(3):493-502.

\section{Grant 2015}

Grant JE, Odlaug BL. Silymarin treatment of obsessivecompulsive spectrum disorders. Journal of Clinical Psychopharmacology 2015;35(3):340-2.

\section{Grant 2016}

Grant JE, Chamberlain SR. Trichotillomania. American Journal of Psychiatry 2016;173(9):868-74.

\section{Guy 1976}

Guy W. ECDEU Assessment Manual for Psychopharmacology. Washington (DC): US National Institute of Health, 1976.

\section{Hamilton 1960}

Hamilton M. A rating scale for depression. Journal of Neurology, Neurosurgery \& Psychiatry 1960;23:56-62.

\section{Higgins 2003}

Higgins JT, Thompson SG, Deeks JJ, Altman DG. Measuring inconsistency in meta-analyses. BMJ 2003;327(6):557-60.

\section{Higgins 2011}

Higgins JP, Green S (editors). Cochrane Handbook for Systematic Reviews of Interventions Version 5.1.0 (updated March 2011). The Cochrane Collaboration, 2011. Available from www.cochrane-handbook.org.

\section{Houghton 2018}

Houghton DC, Alexander JR, Bauer CC, Woods DW. Bodyfocused repetitive behaviors: more prevalent than once thought? Psychiatry Research 2018;270:389-93.

\section{Iancu 1996}

lancu I, Weizman A, Kindler S, Sasson Y, Zohar J. Serotonergic drugs in trichotillomania. Journal of Nervous and Mental Disease 1996;184(10):641-4.

\section{Ikemoto 2010}

Ikemoto S. Brain reward circuitry beyond the mesolimbic dopamine system: a neurobiological theory. Neuroscience \& Biobehavioral Reviews 2010;35(2):129-50.

\section{Jafferany 2018}

Jafferany M, Patel A. Therapeutic aspects of trichotillomania: a review of current treatment options. Primary Care Companion to CNS Disorders 2018;20(6):18nr02344.

\section{Keuthen 1995}

Keuthen NJ, O'Sullivan RL, Ricciardi JN, Shera D, Savage CR, Borgmann AS, et al. The Massachusetts General Hospital (MGH) hairpulling scale: 1 . development and factor analyses. Psychotherapy and Psychosomatics 1995;64(3-4):141-5.

\section{Klipstein 2012}

Klipstein KG, Berman L. Bupropion XL for the sustained treatment of trichotillomania. Journal of Clinical Psychopharmacology 2012;32(2):298-9.

\section{Koran 1992}

Koran LM, Ringold A, Hewlett W. Fluoxetine for trichotillomania: an open clinical trial. Psychopharmacology Bulletin 1992;28(22):145-9.

\section{Lamothe 2019}

Lamothe H, Baleyte JM, Mallet L, Pelissolo A. Trichotillomania is more related to Tourette disorder than to obsessive-compulsive disorder. Brazilian Journal of Psychiatry 2019;42(1):87-104.

\section{Lee 2019}

Lee MT, Fineberg NA, Mpavaenda DN. Habit reversal therapy in obsessive compulsive related disorders: a systematic review of the evidence and consort evaluation of randomized controlled trials. Frontiers in Behavioral Neuroscience 2019;13:79.

\section{Leombruni 2010}

Leombruni P, Gastaldi F. Oxcarbazepine for the treatment of trichotillomania. Clinical Neuropharmacology 2010;33(2):107-8.

\section{Lochner 2006}

Lochner C, Seedat S, Niehaus DJ, Stein DJ. Topiramate in the treatment of trichotillomania: an open-label pilot study. International Clinical Psychopharmacology 2006;21(5):255-9.

\section{Lochner 2019}

Lochner C, Keuthen NJ, Curley EE, Tung ES, Redden SA, Ricketts EJ, et al. Comorbidity in trichotillomania (hair-pulling disorder): a cluster analytical approach. Brain and Behavior $2019 ; 9$ (12):e01456.

\section{Lu 2010}

Lu P, Mamiya T, Lu L, Mouri A, Niwa M, Kim HC, et al. Silibinin attenuates cognitive deficits and decreases of dopamine and serotonin induced by repeated methamphetamine treatment. Behavioural Brain Research 2010;207(2):387-93.

\section{Mahr 1993}

Mahr G. Fenfluramine and trichotillomania. Psychosomatics 1993;34(3):284-84.

\section{Maj 1982}

Maj J, Stala L, Górka Z, Adamus A. Comparison of the pharmacological actions of desmethylclomipramine and clomipramine. Psychopharmacology $1982 ; 78(2): 165-9$. 


\section{Marshall 2000}

Marshall M, Lockwood A, Bradley C, Adams C, Joy C, Fenton M. Unpublished rating scales: a major source of bias in randomised controlled trials of treatments for schizophrenia. British Journal of Psychiatry 2000;176:249-52.

\section{McGuire 2014}

McGuire JF, Ung D, Selles RR, Rahman O, Lewin AB, Murphy TK, et al. Treating trichotillomania: a meta-analysis of treatment effects and moderators for behavior therapy and serotonin reuptake inhibitors. Journal of Psychiatric Research 2014;58:76-83.

\section{Montgomery 1979}

Montgomery SA, Asberg M. A new depression scale designed to be sensitive to change. British Journal of Psychiatry 1979;134:382-9.

\section{Odlaug 2007}

Odlaug BL, Grant JE. N-acetyl cysteine in the treatment of grooming disorders. Journal of Clinical Psychopharmacology 2007;27(2):227-9.

\section{Odlaug 2010}

Odlaug BL, Grant JE. Impulse-control disorders in a college sample: results from the self-administered Minnesota Impulse Disorders Interview (MIDI). Primary Care Companion Journal of Clinical Psychiatry 2010;12(2):PCC.09m00842.

\section{Odlaug 2012}

Odlaug BL, Chamberlain SR, Harvanko AM, Grant JE. Age at onset in trichotillomania: clinical variables and neurocognitive performance. Primary Care Companion to CNS Disorders 2012;14(4):PCC.12m01343. [DOI: 10.4088/PCC.12m01343]

\section{Pollard 1991}

Pollard CA, Ibe IO, Krojanker DN, Kitchen AD, Bronson SS Flynn TM. Clomipramine treatment of trichotillomania: a follow-up report on four cases. Journal of Clinical Psychiatry 1991;52(3):128-30.

\section{Rehm 2015}

Rehm I, Moulding R, Nedeljkovic M. Psychological treatments for trichotillomania: update and future directions. Australasian Psychiatry 2015;23(4):365-8.

\section{Review Manager 2014 [Computer program]}

Nordic Cochrane Centre,The Cochrane Collaboration Review Manager 5 (RevMan 5). Version 5.3. Copenhagen: Nordic Cochrane Centre,The Cochrane Collaboration, 2014.

\section{Rodrigues-Barata 2012}

Rodrigues-Barata AR, Tosti A, Rodríguez-Pichardo A, Camacho-Martínez F. N-acetylcysteine in the treatment of trichotillomania. International Journal of Trichology 2012;4(3):176.

\section{Schünemann 2013}

Schünemann H, Brożek J, Guyatt G, Oxman A, editors. Handbook for grading the quality of evidence and strength of recommendations using the GRADE approach (updated
October 2013). GRADE Working Group, 2013. Available from gdt.guidelinedevelopment.org/app/handbook/handbook.html.

\section{Sentürk 2002}

Sentürk V, Tanriverdi N. Resistant trichotillomania and risperidone. Psychosomatics 2002;43(5):429-30.

\section{Sharma 2008}

Sharma V, Corpse C. Lithium treatment of trichotillomania with comorbid bipolar II disorder. Archives of Women's Mental Health 2008;11(4):305-6.

\section{Sheehan 1996}

Sheehan DV, Harnett-Sheehan K, Raj BA. The measurement of disability. International Clinical Psychopharmacology 1996;11(Suppl 3):89-95

\section{Silva-Netto 2014}

Silva-Netto R, Jesus G, Nogueira M, Tavares H. N-acetylcysteine in the treatment of skin-picking disorder. Brazilian Journal of Psychiatry 2014;36(1):101.

\section{Song 2000}

Song F, Eastwood AJ, Gilbody S, Duley L, Sutton AJ. Publication and related biases. Health Technology Assessment 2000;4:1-115.

\section{Spitzer 1996}

Spitzer R, Williams J, Gibbons M. Instructional Manual for the Structured Clinical Interview for DSM-IV. New York (NY): New York State Psychiatric Institute, 1996.

\section{Stahl 2008}

Stahl SM. Stahl's Essential Psychopharmacology: Neuroscientific Basis and Practical Applications. 3rd edition. Cambridge (UK): Cambridge University Press, 2008.

\section{Stanley 1991}

Stanley MA, Bowers TC, Swann AC, Taylor DJ. Treatment of trichotillomania with fluoxetine. Journal of Clinical Psychiatry 1991;52(6):282.

\section{Stein 2002}

Stein DJ, Van Heerden B, Hugo C, Van Kradenburg J, Warwick J, Zungu-Dirwayi N, et al. Functional brain imaging and pharmacotherapy in trichotillomania single photon emission computed tomography before and after treatment with the selective serotonin reuptake inhibitor citalopram. Progress in Neuro-Psychopharmacology and Biological Psychiatry 2002;26:885-90.

\section{Stein 2006}

Stein DJ, Chamberlain SR, Fineberg N. An ABC model of habit disorders: hair-pulling, skin-picking, and other stereotypic conditions. CNS Spectrums 2006;11(11):824-7.

\section{Stein 2010}

Stein DJ, Grant JE, Franklin ME, Keuthen N, Lochner C, Singer HS, et al. Trichotillomania (hair pulling disorder), skin picking disorder, and stereotypic movement disorder: toward DSM-V. Depression and Anxiety 2010;27:611-26. 


\section{Stewart 2003}

Stewart RS, Nejtek VA. An open-label, flexible dose study of olanzapine in the treatment of trichotillomania. Journal of Clinical Psychiatry 2003;64(1):49-52.

\section{Swedo 1992}

Swedo SE, Leonard HL. Trichotillomania: an obsessive compulsive spectrum disorder? Psychiatric Clinics of North America 1992;15(4):777-90.

\section{Taylor 2014}

Taylor M, Bhagwandas K. N-acetylcysteine in trichotillomania: a panacea for compulsive skin disorders? British Journal of Dermatology 2014;171(5):1253-5.

\section{Van Ameringen 1999}

Van Ameringen M, Mancini C, Oakman JM, Farvolden P. The potential role of haloperidol in the treatment of trichotillomania. Journal of Affective Disorders 1999;56:219-26.

\section{Van der Stelt 2005}

Van der Stelt M, Di Marzo V. Cannabinoid receptors and their role in neuroprotection. Neuromolecular Medicine 2005;7(1-2):37-50.

\section{Verbeke 2000}

Verbeke M, Molenberghs G. Linear Mixed Models for Longitudinal Data. New York (NY): Springer, 2000.

\section{White 2011}

White MP, Koran LM. Open-label trial of aripiprazole in the treatment of trichotillomania. Journal of Clinical Psychopharmacology 2011;31(4):503-6.

\section{WHO 2018}

World Health Organization. International classification of diseases for mortality and morbidity statistics (11th Revision).

\section{CHARACTERISTICS OF STUDIES}

Characteristics of included studies [ordered by study ID] https://icd.who.int/browse11/l-m/en (accessed prior to 8 September 2021).

\section{Winchel 1992}

Winchel RM, Jones JS, Stanley B, Molcho A, Stanley M. Clinical characteristics of trichotillomania and its response to fluoxetine. Journal of Clinical Psychiatry 1992;53(9):304-8.

\section{Woods 2006}

Woods DW, Flessner CA, Franklin ME, Keuthen NJ, Goodwin RD, Stein DJ, et al, Trichotillomania Learning Center Advisory Board. The Trichotillomania Impact Project (TIP): exploring phenomenology, functional impairment, and treatment utilization. Journal of Clinical Psychiatry 2006;67:1877-88.

\section{Yasui-Furukori 2011}

Yasui-Furukori N, Kaneko S. The efficacy of low-dose aripiprazole treatment for trichotillomania. Clinical Neuropharmacology 2011;34(6):258-9.

\section{Youssef 2008}

Youssef NCM, Reinhart K, Sakr Y. The pros and cons of multicentre studies. Netherlands Journal of Critical Care 2008;12(3):120-2.

\section{References to other published versions of this review Rothbart 2013 \\ Rothbart R, Amos T, Siegfried N, Ipser JC, Fineberg N, Chamberlain SR, et al. Pharmacotherapy for trichotillomania. Cochrane Database of Systematic Reviews 2013, Issue 11. Art. No: CD007662. [DOI: 10.1002/14651858.CD007662.pub2]}

* Indicates the major publication for the study

\section{Bloch 2013}

\section{Study characteristics}

Methods Randomised controlled parallel-arm trial, 12 weeks

Participants

SAMPLE

39 DSM-IV TTM (criteria B and C not mandatory) with symptoms of hair-pulling for at least 6 months; mean age 13.5 ( \pm 2.8 ) years; 34 females $/ 5$ males; mean $( \pm$ SD) duration of hair-pulling of $4.4( \pm 2.6)$ years in the intervention group and $3.3( \pm 2.4)$ years in the placebo group; baseline severity of TTM on the MGH-HPS was $13.2( \pm 5.3)$ in the intervention group and $16.6( \pm 4.8)$ in the placebo group.

\section{SCREENING}

Primary diagnosis: DSM-IV

Comorbidities: $13(33.3 \%)$ with depression, 9 (23.1\%) with anxiety, $4(10.2 \%)$ with ADHD, $3(7.7 \%)$ with tic disorder, 2 (5.1\%) with OCD and 1 (2.6\%) with skin picking. 
Bloch 2013 (Continued)

Interventions

Description: N-acetylcysteine $(600 \mathrm{mg} / \mathrm{d}$ for first week, titrated over 4 weeks to $2400 \mathrm{mg} / \mathrm{d}$ for the remaining 8 weeks) versus placebo $\times 12$ weeks

Outcomes

Primary outcome measures: MGH-HPS.

Secondary outcome measures: TSC-C, TSC-P, NIMH-TSS, MASC, CDI, and CGI-I.

Notes INDUSTRY SUPPORT

Industry-funded: no, the Trichotillomania Learning Center helped fund the study (from published protocol).

Medication provided by industry: unclear

Any of the authors work for industry: no (from conflicts of interest section and published protocol)

\section{Risk of bias}

\begin{tabular}{lll}
\hline Bias & Authors' judgement & Support for judgement \\
\hline $\begin{array}{ll}\text { Random sequence genera- } \\
\text { tion (selection bias) }\end{array}$ & Unclear risk & $\begin{array}{l}\text { It was reported that the participants were randomised; however, the method } \\
\text { of randomisation is unclear. Quote: "Subjects were randomized in a ratio of 1:1 } \\
\text { to receive treatment with NAC (Swanson Nutraceuticals) or placebo". }\end{array}$ \\
\hline
\end{tabular}

$\begin{array}{ll}\begin{array}{l}\text { Allocation concealment } \\ \text { (selection bias) }\end{array} & \text { Low risk } \\ & \begin{array}{l}\text { Pharmacy-controlled allocation. Quote: "Randomization data were kept by the } \\ \text { investigational pharmacist and were kept strictly confidential until the time of } \\ \text { unblinding, after all study assessments were complete". }\end{array}\end{array}$

Blinding (performance Low risk

bias and detection bias)

Self-reported outcomes
Participants were blinded throughout the study. Additional efforts were made to conceal the identity of the active agent and placebo, such as masking the smell of both with peppermint and keeping the placebo pills with active NAC pills to confer the same distinctive aroma to each tablet. Quote: "Subjects, their parents, investigators, and persons performing the assessments remained blind to treatment assignment from the time of randomization until the completion of the study. Adequacy of blinding was assessed by asking both subjects and the treating clinician, at the end of the trial, whether they thought they were assigned to NAC or placebo. Neither subjects $(x 2=0.01, p$ $=0.92)$ or treating clinicians $(x 2=1.4, p=0.24)$ were significantly better than chance when determining treatment allocation."

\begin{tabular}{|c|c|c|}
\hline $\begin{array}{l}\text { Blinding (performance } \\
\text { bias and detection bias) }\end{array}$ & Low risk & $\begin{array}{l}\text { All study personnel, including outcome assessors, were blinded throughout } \\
\text { the study. See quote above. }\end{array}$ \\
\hline
\end{tabular}

Objective outcomes

\begin{tabular}{ll}
\hline $\begin{array}{l}\text { Incomplete outcome data } \\
\text { (attrition bias) }\end{array}$ & Low risk \\
Self-reported outcomes & $\begin{array}{l}\text { More participants withdrew from the N-acetylcysteine group (4/20; 20\%) com- } \\
\text { pared to the placebo }(0 / 19 ; 0 \%) \text { group. Reasons for withdrawal include diffuse } \\
\text { rash, change in dosage of concomitant medication and non-compliance with } \\
\text { study medication. Intention-to-treat analysis with last observation carried for- } \\
\text { ward was used, including the } 4 \text { non-completers in the analysis. }\end{array}$
\end{tabular}

Incomplete outcome data Low risk As above

(attrition bias)

Objective outcomes
Additional outcomes reported on in the publication compared to protocol but no change to overall findings. All outcomes in protocol reported in publication. 
Bloch 2013 (Continued)

Other bias Unclear risk High percentage of female participants. Concomitant psychotropic medication use. Quote: "41\% [of participants] were concurrently taking SSRIs while enrolled in our trial".

Christenson 1991a

\section{Study characteristics}

\begin{tabular}{|c|c|}
\hline Methods & Randomised controlled cross-over trial, 6 weeks with 5-week washout period \\
\hline \multirow[t]{6}{*}{ Participants } & SAMPLE \\
\hline & $\begin{array}{l}16 \text { DSM-III TTM (criteria B and C not mandatory) with at least } 3 \text { hair-pulling episodes per week; mean } \\
\text { age } 31.6 \text { ( } 22 \text { to } 45 \text { ) years; } 15 \text { females/1 male; mean duration of hair-pulling: } 17 \text { ( } 7 \text { to } 33 \text { ) years; baseline } \\
\text { severity of TTM not stated; comorbidities: } 5 \text { MDD, } 5 \text { GAD, } 2 \text { social phobia, } 1 \text { agoraphobia, } 1 \text { simple pho- } \\
\text { bia, } 3 \text { eating disorders }\end{array}$ \\
\hline & SCREENING \\
\hline & $\begin{array}{l}\text { Primary diagnosis: Minnesota Trichotillomania Assessment Inventory (90-minute semistructured inter- } \\
\text { view) }\end{array}$ \\
\hline & One-week assessment of baseline hair-pulling ( $<3$ hair-pulling episodes/week not eligible) \\
\hline & Comorbidities: NIMH DIS \\
\hline Interventions & Description: fluoxetine $(20$ to $80 \mathrm{mg} / \mathrm{d}$ ) versus placebo $\times$ six weeks \\
\hline \multirow[t]{2}{*}{ Outcomes } & $\begin{array}{l}\text { Primary outcomes measures: number of hair-pulling episodes/week, estimated number of hairs pulled/ } \\
\text { week, counted number of hairs pulled/week, rating of severity of the urge to pull hair/week, rating of } \\
\text { severity of hair-pulling/week }\end{array}$ \\
\hline & Secondary outcomes measures: HAM-D, BDI \\
\hline \multirow[t]{4}{*}{ Notes } & INDUSTRY SUPPORT \\
\hline & Industry-funded: no (from correspondence with the first author) \\
\hline & Medication provided by industry: no (from correspondence with the first author) \\
\hline & Any of the authors work for industry: no (from correspondence with the first author) \\
\hline
\end{tabular}

\section{Risk of bias}

\begin{tabular}{lll}
\hline Bias & Authors' judgement & Support for judgement \\
\hline $\begin{array}{l}\text { Random sequence genera- } \\
\text { tion (selection bias) }\end{array}$ & Low risk & Computerised randomisation (from correspondence with the first author) \\
\hline $\begin{array}{l}\text { Allocation concealment } \\
\text { (selection bias) }\end{array}$ & Low risk & Pharmacy-controlled allocation (from correspondence with the first author) \\
\hline $\begin{array}{l}\text { Blinding (performance } \\
\text { bias and detection bias) }\end{array}$ & Low risk & $\begin{array}{l}\text { Participants were blinded throughout the study (from correspondence with } \\
\text { the first author) }\end{array}$ \\
\hline $\begin{array}{l}\text { Blinding (performance } \\
\text { bias and detection bias) }\end{array}$ & Low risk & $\begin{array}{l}\text { All study personnel, including outcome assessors, were blinded throughout } \\
\text { the study (from correspondence with the first author) }\end{array}$ \\
\hline
\end{tabular}


Christenson 1991a (Continued)

Objective outcomes

\begin{tabular}{|c|c|c|}
\hline $\begin{array}{l}\text { Incomplete outcome data } \\
\text { (attrition bias) } \\
\text { Self-reported outcomes }\end{array}$ & Low risk & $\begin{array}{l}\text { One participant was dropped from the study because of failure to keep ap- } \\
\text { pointments and these data were excluded from the analysis. The participant } \\
\text { reported complete resolution of symptoms on day } 2 \text { of the placebo regimen, } \\
\text { which was maintained for } 2 \text { weeks into the fluoxetine treatment period ( } 1 / 17= \\
6 \% \text { attrition rate). }\end{array}$ \\
\hline
\end{tabular}

Incomplete outcome data Low risk As above
(attrition bias)

Objective outcomes

\begin{tabular}{lll}
\hline $\begin{array}{l}\text { Selective reporting (re- } \\
\text { porting bias) }\end{array}$ & Unclear risk & $\begin{array}{l}\text { The 'number of hairs pulled' was not included in the outcome analysis be- } \\
\text { cause collecting and counting individual hairs was found to be too cumber- } \\
\text { some. The reason for these missing outcome data is not likely to be related to } \\
\text { true outcome. Rated as unclear risk of reporting bias because a trial protocol } \\
\text { was not obtained. }\end{array}$ \\
\hline Other bias & Low risk & $\begin{array}{l}\text { High percentage of female participants. We did not find any other source of } \\
\text { bias. }\end{array}$ \\
\hline
\end{tabular}

\section{Christenson 1994}

\section{Study characteristics}

\begin{tabular}{|c|c|c|}
\hline Methods & \multicolumn{2}{|c|}{ Randomised controlled parallel-arm trial, 6 weeks } \\
\hline \multirow[t]{4}{*}{ Participants } & \multicolumn{2}{|l|}{ SAMPLE } \\
\hline & \multicolumn{2}{|l|}{17 individuals with TTM } \\
\hline & \multicolumn{2}{|l|}{ SCREENING } \\
\hline & \multicolumn{2}{|c|}{ Information about screening not available } \\
\hline Interventions & \multicolumn{2}{|c|}{ Description: naltrexone $(50 \mathrm{mg} / \mathrm{d})$ versus placebo $\times 6$ weeks } \\
\hline \multirow[t]{2}{*}{ Outcomes } & \multicolumn{2}{|c|}{$\begin{array}{l}\text { Primary outcome measures: NIMH-TSS, NIMH-TIS, number of hair-pulling episodes, estimated number } \\
\text { of hairs pulled. }\end{array}$} \\
\hline & \multicolumn{2}{|c|}{ Secondary outcome measures: none } \\
\hline Notes & \multicolumn{2}{|c|}{$\begin{array}{l}\text { Principal investigator was contacted for more information, but he no longer had information about this } \\
\text { trial. }\end{array}$} \\
\hline \multicolumn{3}{|l|}{ Risk of bias } \\
\hline Bias & Authors' judgement & Support for judgement \\
\hline $\begin{array}{l}\text { Random sequence genera- } \\
\text { tion (selection bias) }\end{array}$ & Low risk & $\begin{array}{l}\text { Earlier reports and correspondence from the same principal investigator clear- } \\
\text { ly describe use of a random sequence. }\end{array}$ \\
\hline $\begin{array}{l}\text { Allocation concealment } \\
\text { (selection bias) }\end{array}$ & Low risk & $\begin{array}{l}\text { Earlier reports and correspondence from the same principal investigator de- } \\
\text { scribe the use of pharmacy-controlled allocation. }\end{array}$ \\
\hline $\begin{array}{l}\text { Blinding (performance } \\
\text { bias and detection bias) }\end{array}$ & Low risk & $\begin{array}{l}\text { Earlier reports and correspondence from the same principal investigator de- } \\
\text { scribe blinding of participants throughout the study. }\end{array}$ \\
\hline
\end{tabular}


Christenson 1994 (Continued)

Self-reported outcomes

\begin{tabular}{|c|c|c|}
\hline $\begin{array}{l}\text { Blinding (performance } \\
\text { bias and detection bias) }\end{array}$ & Low risk & $\begin{array}{l}\text { Earlier reports and correspondence from the same principal investigator de- } \\
\text { scribe blinding of study personnel throughout the study. }\end{array}$ \\
\hline
\end{tabular}

Objective outcomes

Incomplete outcome data Unclear risk Insufficient information to permit judgement
(attrition bias)

Self-reported outcomes

\begin{tabular}{lll}
\hline $\begin{array}{l}\text { Incomplete outcome data } \\
\text { (attrition bias) } \\
\text { Objective outcomes }\end{array}$ & Unclear risk & Insufficient information to permit judgement \\
\hline $\begin{array}{l}\text { Selective reporting (re- } \\
\text { porting bias) }\end{array}$ & Unclear risk & Insufficient information to permit judgement (trial protocol not obtained) \\
\hline Other bias & Low risk & No other sources of bias found \\
\hline
\end{tabular}

Dougherty 2006

\section{Study characteristics}

Rethods
Randomised controlled parallel-arm trial, 12 weeks
added to their treatment regimen for an additional 8 weeks. Outcomes on individuals given single ther-
apy with sertraline were compared with those receiving dual therapy (sertraline and HRT). Only data
from the first phase of the study (first 12 weeks) comparing sertraline with placebo were included in our
analysis.

\section{Participants}

\section{SAMPLE}

37 DSM-IV TTM, 19 in sertraline arm/18 in placebo arm, with symptoms for at least 4 months, with the scalp as the primary site of hair-pulling and with an MGH-HPS score $\geq 15$ or a TTMIS score $>30$. More than $90 \%$ female participants.

\section{SCREENING}

Primary diagnosis: SCID

Comorbidities: no screening for comorbidities described

\begin{tabular}{ll}
\hline Interventions & Description: fluoxetine $(12 \mathrm{mg}$ to $200 \mathrm{mg} / \mathrm{d}$ ) versus placebo $\times 12$ weeks \\
\hline Outcomes & Primary outcome measures: MGH-HPS, PITS, TTMIS. \\
& Secondary outcome measures: CGI-I, HAM-D, BDI, BAI, Q-LES-Q. \\
\hline Notes & INDUSTRY SUPPORT \\
& Industry-funded: yes (from correspondence with the first author) \\
& Medication provided by industry: yes (from correspondence with the first author) \\
& Any of the authors work for industry: no (from correspondence with the first author)
\end{tabular}

\section{Risk of bias}


Dougherty 2006 (Continued)

\begin{tabular}{|c|c|c|}
\hline Bias & Authors' judgement & Support for judgement \\
\hline $\begin{array}{l}\text { Random sequence genera- } \\
\text { tion (selection bias) }\end{array}$ & Low risk & Computerised randomisation (from correspondence with the first author) \\
\hline $\begin{array}{l}\text { Allocation concealment } \\
\text { (selection bias) }\end{array}$ & Low risk & Pharmacy-controlled allocation (from correspondence with the first author) \\
\hline $\begin{array}{l}\text { Blinding (performance } \\
\text { bias and detection bias) } \\
\text { Self-reported outcomes }\end{array}$ & Low risk & $\begin{array}{l}\text { Participants were blinded throughout the study and the blind was never bro- } \\
\text { ken (from correspondence with the first author) }\end{array}$ \\
\hline $\begin{array}{l}\text { Blinding (performance } \\
\text { bias and detection bias) } \\
\text { Objective outcomes }\end{array}$ & Low risk & $\begin{array}{l}\text { All study personnel, including outcome assessors, were blinded throughout } \\
\text { the study and the blind was never broken (from correspondence with the first } \\
\text { author). }\end{array}$ \\
\hline $\begin{array}{l}\text { Incomplete outcome data } \\
\text { (attrition bias) } \\
\text { Self-reported outcomes }\end{array}$ & High risk & $\begin{array}{l}37 \text { participants were randomly assigned in the study; however, intention-to- } \\
\text { treat analysis was not used, as data from only } 31 \text { participants were analysed } \\
\text { (sertraline group } 4 / 19=21 \% \text { attrition rate and placebo group } 2 / 18=11 \% \text { attri- } \\
\text { tion rate). Reasons for attrition were not given }\end{array}$ \\
\hline $\begin{array}{l}\text { Incomplete outcome data } \\
\text { (attrition bias) } \\
\text { Objective outcomes }\end{array}$ & High risk & As above \\
\hline $\begin{array}{l}\text { Selective reporting (re- } \\
\text { porting bias) }\end{array}$ & Unclear risk & Insufficient information to permit judgement (trial protocol not obtained) \\
\hline Other bias & Unclear risk & $\begin{array}{l}\text { Industry-funded and medication provided by industry. High percentage of fe- } \\
\text { male participants. }\end{array}$ \\
\hline
\end{tabular}

Grant 2009

\section{Study characteristics}

\begin{tabular}{|c|c|}
\hline Methods & Randomised controlled parallel-arm trial, 12 weeks \\
\hline \multirow[t]{5}{*}{ Participants } & SAMPLE \\
\hline & $\begin{array}{l}50 \text { DSM-IV TTM (criteria B and C not mandatory), } 25 \text { participants in NAC arm/25 in placebo arm; mean } \\
\text { age } 34.3 \text { (18 to 59) years, } 45 \text { females/5 males; mean age of onset } 12.1 \text { ( } 3 \text { to } 27 \text { ) years; comorbidities: } 28 \% \\
\text { MDD, } 28 \% \text { anxiety disorder, } 36 \% \text { another impulse-control disorder, } 2 \% \text { eating disorder. }\end{array}$ \\
\hline & SCREENING \\
\hline & $\begin{array}{l}\text { Primary diagnosis: Trichotillomania Diagnostic Interview (to confirm that participants meet DSM-IV cri- } \\
\text { teria). }\end{array}$ \\
\hline & Comorbidities: SCID. \\
\hline Interventions & $\begin{array}{l}\text { Description: N-acetylcysteine }(1200 \text { mg/d for first six weeks, then } 2400 \mathrm{mg} / \mathrm{d} \text { ) versus placebo × } 12 \\
\text { weeks }\end{array}$ \\
\hline \multirow[t]{2}{*}{ Outcomes } & Primary outcomes: MGH-HPS. \\
\hline & Secondary outcomes: PITS, CGI-I, CGI-S, HAM-A, HAM-D, QoLI, SDS. \\
\hline
\end{tabular}


Grant 2009 (Continued)

Notes
INDUSTRY SUPPORT
Industry-funded: no
Medication provided by industry: no
Any of the authors work for industry: yes, as a consultant

\section{Risk of bias}

\begin{tabular}{|c|c|c|}
\hline Bias & Authors' judgement & Support for judgement \\
\hline $\begin{array}{l}\text { Random sequence genera- } \\
\text { tion (selection bias) }\end{array}$ & Low risk & Computer-generated randomisation \\
\hline $\begin{array}{l}\text { Allocation concealment } \\
\text { (selection bias) }\end{array}$ & Low risk & Pharmacy-controlled allocation (from correspondence with the first author) \\
\hline $\begin{array}{l}\text { Blinding (performance } \\
\text { bias and detection bias) } \\
\text { Self-reported outcomes }\end{array}$ & Low risk & $\begin{array}{l}\text { Participants were blinded throughout the study (from correspondence with } \\
\text { the first author) }\end{array}$ \\
\hline $\begin{array}{l}\text { Blinding (performance } \\
\text { bias and detection bias) } \\
\text { Objective outcomes }\end{array}$ & Low risk & $\begin{array}{l}\text { All study personnel, including outcome assessors, were blinded throughout } \\
\text { the study (from correspondence with the first author) }\end{array}$ \\
\hline $\begin{array}{l}\text { Incomplete outcome data } \\
\text { (attrition bias) } \\
\text { Self-reported outcomes }\end{array}$ & Low risk & $\begin{array}{l}\text { Intention-to-treat analysis was used and last observation carried forward for } 6 \\
\text { non-completers ( } 3 \text { from the placebo group, } 3 \text { from the intervention group) }\end{array}$ \\
\hline $\begin{array}{l}\text { Incomplete outcome data } \\
\text { (attrition bias) } \\
\text { Objective outcomes }\end{array}$ & Low risk & $\begin{array}{l}\text { Intention-to-treat analysis was used and last observation carried forward for } 6 \\
\text { non-completers ( } 3 \text { from the placebo group, } 3 \text { from the intervention group) }\end{array}$ \\
\hline $\begin{array}{l}\text { Selective reporting (re- } \\
\text { porting bias) }\end{array}$ & Low risk & $\begin{array}{l}\text { Additional outcomes reported on in the publication compared to protocol but } \\
\text { no new significant results. All outcomes in protocol reported in publication. }\end{array}$ \\
\hline Other bias & Unclear risk & $\begin{array}{l}\text { Author worked as a consultant for pharmaceutical company. High percentage } \\
\text { of female participants. }\end{array}$ \\
\hline
\end{tabular}

Grant 2014

\section{Study characteristics}

\begin{tabular}{ll}
\hline Methods & Randomised, double-blind, placebo-controlled parallel-arm trial, 8 weeks \\
\hline Participants & SAMPLE \\
& 51 DSM-IVTTM, 25 participants in naltrexone arm/26 in placebo arm; mean age 32.7 years (SD 9.8), 44 \\
females/7 males; mean age of onset 13.7 years (4 to 45 years); comorbidities: $52.9 \%$ of participants re- \\
ported a comorbidity, no further details. Three (5.9\%) had ongoing psychotherapy, 17 (33.\%) were tak- \\
ing a psychotropic medication. No difference between treatment groups were reported. \\
SCREENING \\
Primary diagnosis: MGH-HPS. "Only subjects who reported urges to pull at least $50 \%$ of the time and a \\
Score greater than or equal to 1 on each of the first three items on the MGH-HPS were included".
\end{tabular}


Grant 2014 (Continued)

Comorbidities: SCID for DSM-IV

Interventions Naltrexone (50 mg per day up to $150 \mathrm{mg}$ per day) versus placebo for 8 weeks

Outcomes Primary outcome measures: MGH-HPS

Secondary outcome measures: CGI-I, CGI-S, HAM-A, HAM-D, NIMH-TSS, QoLI, SDS.

Cognition: intradimensional/extradimensional shift task and SST.

$\begin{array}{ll}\text { Notes } & \text { INDUSTRY SUPPORT } \\ \text { Industry-funded: no } \\ \text { Medication provided by industry: no } \\ \text { Any of the authors work for industry: no }\end{array}$

\section{Risk of bias}

\begin{tabular}{|c|c|c|}
\hline Bias & Authors' judgement & Support for judgement \\
\hline $\begin{array}{l}\text { Random sequence genera- } \\
\text { tion (selection bias) }\end{array}$ & Low risk & $\begin{array}{l}\text { Quote: "Subjects were randomized in block sizes of } 8 \text {, using computer-gener- } \\
\text { ated randomization with no clinical information in a } 1: 1 \text { fashion." }\end{array}$ \\
\hline $\begin{array}{l}\text { Allocation concealment } \\
\text { (selection bias) }\end{array}$ & Unclear risk & $\begin{array}{l}\text { Quote: "All eligible study subjects were started on naltrexone } 50 \mathrm{mg} / \mathrm{d} \text { or } \\
\text { matching placebo for } 2 \text { weeks" (no further details available). }\end{array}$ \\
\hline $\begin{array}{l}\text { Blinding (performance } \\
\text { bias and detection bias) } \\
\text { Self-reported outcomes }\end{array}$ & Low risk & $\begin{array}{l}\text { Participants were blinded throughout the study period as per the study proto- } \\
\text { col on ClinicalTrials.gov. }\end{array}$ \\
\hline $\begin{array}{l}\text { Blinding (performance } \\
\text { bias and detection bias) } \\
\text { Objective outcomes }\end{array}$ & Low risk & $\begin{array}{l}\text { Outcomes assessors reported as blinded throughout the study period as per } \\
\text { the study protocol on ClinicalTrials.gov. }\end{array}$ \\
\hline $\begin{array}{l}\text { Incomplete outcome data } \\
\text { (attrition bias) } \\
\text { Self-reported outcomes }\end{array}$ & Low risk & $\begin{array}{l}\text { More participants withdrew from the naltrexone group }(5 / 25 ; 20 \%) \text { compared } \\
\text { to the placebo }(2 / 26 ; 8 \%) \text { group. Reasons for withdrawal were not specified. } \\
\text { No information was provided on sample characteristics at endpoint; howev- } \\
\text { er, demographics and clinical characteristics of the participants at baseline } \\
\text { are presented, with no apparent difference reported between groups. Inten- } \\
\text { tion-to-treat analysis was done. Quote: "The rate of study completion did not } \\
\text { differ between groups ( } p=0.248) \text {, with } 20(80 \%) \text { of } 25 \text { subjects assigned to nal- } \\
\text { trexone, and } 24(92.3 \%) \text { of } 26 \text { subjects assigned to placebo completing the } 8 \text { - } \\
\text { week trial. There were no statistically significant pre-treatment differences be- } \\
\text { tween completers and non-completers on any measures." }\end{array}$ \\
\hline $\begin{array}{l}\text { Incomplete outcome data } \\
\text { (attrition bias) } \\
\text { Objective outcomes }\end{array}$ & Low risk & As above \\
\hline $\begin{array}{l}\text { Selective reporting (re- } \\
\text { porting bias) }\end{array}$ & Low risk & $\begin{array}{l}\text { All outcomes in protocol reported on and additional outcomes added in the } \\
\text { paper. Additional outcomes did not influence the conclusions drawn. }\end{array}$ \\
\hline Other bias & Low risk & $\begin{array}{l}\text { High percentage of female participants compared to male, concomitant psy- } \\
\text { chotropic medication use. No significant differences between naltrexone and } \\
\text { placebo groups. }\end{array}$ \\
\hline
\end{tabular}


Grant 2019

\section{Study characteristics}

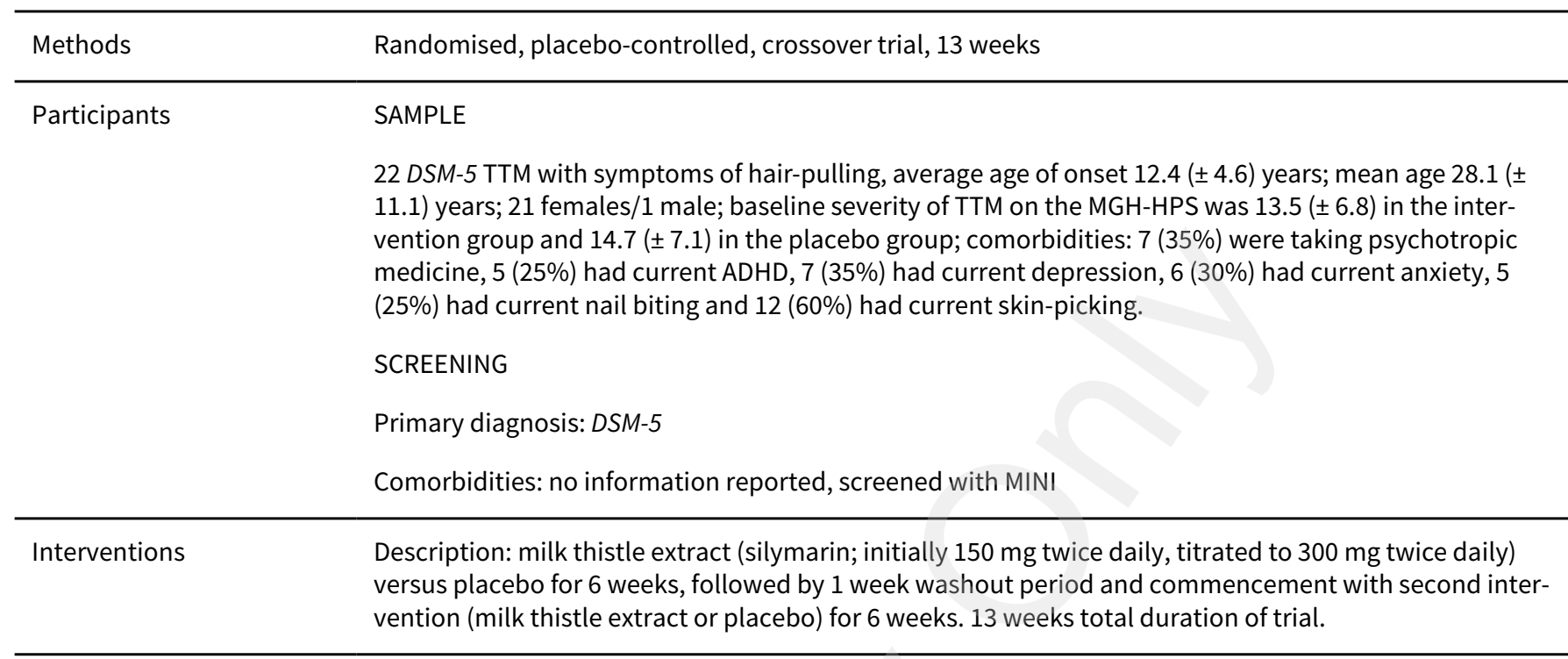

Outcomes $\quad$ Primary outcome measures: NIMH-TSS.

Secondary outcome measures: MGH-HPS, CGI-S, CGI-I, SDS, HAM-A, HAM-D, and TSC-C,P.

Notes INDUSTRY SUPPORT

Industry-funded: no, funded by internal departmental funds of the Department of Psychiatry at the University of Chicago (from published protocol).

Medication provided by industry: unclear

Any of the authors work for industry: no (from conflicts of interest section and published protocol)

\section{Risk of bias}

\begin{tabular}{|c|c|c|}
\hline Bias & Authors' judgement & Support for judgement \\
\hline $\begin{array}{l}\text { Random sequence genera- } \\
\text { tion (selection bias) }\end{array}$ & Low risk & $\begin{array}{l}\text { Quote: "All blinding and assignment was conducted using a randomization } \\
\text { code provided by a pharmacy independent of the research team". }\end{array}$ \\
\hline $\begin{array}{l}\text { Allocation concealment } \\
\text { (selection bias) }\end{array}$ & Low risk & $\begin{array}{l}\text { Quote: "All blinding and assignment was conducted using a randomization } \\
\text { code provided by a pharmacy independent of the research team" and "[t]his } \\
\text { blinding will be maintained by the IDS pharmacy at the University of Chicago." }\end{array}$ \\
\hline $\begin{array}{l}\text { Blinding (performance } \\
\text { bias and detection bias) } \\
\text { Self-reported outcomes }\end{array}$ & Low risk & $\begin{array}{l}\text { Quote: "Subjects will be given a matching placebo during the 6-week placebo } \\
\text { phase" }\end{array}$ \\
\hline $\begin{array}{l}\text { Blinding (performance } \\
\text { bias and detection bias) } \\
\text { Objective outcomes }\end{array}$ & Low risk & $\begin{array}{l}\text { Quote: "all blinding and assignment was conducted using a randomization } \\
\text { code provided by a pharmacy independent of the research team". }\end{array}$ \\
\hline $\begin{array}{l}\text { Incomplete outcome data } \\
\text { (attrition bias) } \\
\text { Self-reported outcomes }\end{array}$ & Low risk & $\begin{array}{l}\text { Reasons for withdrawal stated as "lost to follow up" in results on ClinicalTrial- } \\
\text { s.gov entry. } \\
\text { Equal number of participants withdrew from placebo-first and milk thistle-first } \\
\text { groups. }\end{array}$ \\
\hline
\end{tabular}


No information was provided on sample characteristics at endpoint; however, demographics and clinical characteristics of the participants at baseline are presented. There were also no statistically significant imbalances in demographics or baseline trichotillomania symptoms between treatment groups.

Intention-to-treat analysis was not employed. However, 17/22 participants are included in most analyses on the results posted to ClinicalTrials.gov, which are approximately equivalent to the results in the published paper. There are no stated differences between completers and non-completers.

Incomplete outcome data Low risk See above

(attrition bias)

Objective outcomes

\begin{tabular}{|c|c|c|}
\hline $\begin{array}{l}\text { Selective reporting (re- } \\
\text { porting bias) }\end{array}$ & Low risk & All outcomes in protocol reported in publication. \\
\hline Other bias & Unclear risk & $\begin{array}{l}\text { High percentage of female participants compared to male. No significant dif- } \\
\text { ferences between milk thistle (silymarin) and placebo groups. Expectancy bias } \\
\text { may play a role, yet positive and negative expectancy bias among participants } \\
\text { likely balances out. }\end{array}$ \\
\hline
\end{tabular}

\section{Leppink 2017}

\section{Study characteristics}

\begin{tabular}{|c|c|}
\hline Methods & Randomised, double-blind, placebo-controlled, parallel-arm trial, 10 weeks \\
\hline \multirow[t]{5}{*}{ Participants } & SAMPLE \\
\hline & $\begin{array}{l}38 \text { DSM-VTTM, } 19 \text { in inositol arm/18 in placebo arm; mean age } 28.9 \text { years (SD } 11.4 \text { ), } 35 \text { females/3 males; } \\
\text { mean time since diagnosis } 12.9 \text { years (SD 6.9); comorbidities: } 15.8 \% \text { MDD, } 21.1 \% \text { anxiety disorder, } \\
34.2 \% \text { skin picking disorders, } 13.2 \% \text { ADHD, } 2.6 \% \text { OCD. }\end{array}$ \\
\hline & SCREENING \\
\hline & $\begin{array}{l}\text { Primary diagnosis: MGH-HPS. Quote: "At baseline and at every 2-week visit, participants were assessed } \\
\text { with several measures of severity and symptom change. Participants reported severity of trichotilloma- } \\
\text { nia symptoms using the self-rated MGH-HPS, the primary outcome measure for the study" }\end{array}$ \\
\hline & Comorbidities: SCID for DSM-IV \\
\hline Interventions & Inositol ( $2 \mathrm{~g}$ three times a day titrated up to $6 \mathrm{~g}$ three times a day) versus placebo for 10 weeks \\
\hline Outcomes & $\begin{array}{l}\text { Primary outcome measures: MGH-HPS. } \\
\text { Secondary outcome measures: CGI-I, CGI-S, HAM-A, HAM-D, NIMH-TSS, QoLI, SDS. }\end{array}$ \\
\hline \multirow[t]{4}{*}{ Notes } & INDUSTRY SUPPORT \\
\hline & Industry-funded: no \\
\hline & Medication provided by industry: no \\
\hline & Any of the authors work for industry: yes, authors worked as consultants for pharmaceutical companies \\
\hline
\end{tabular}

\section{Risk of bias}


Leppink 2017 (Continued)

\section{Bias Authors' judgement Support for judgement}

Random sequence genera- Low risk tion (selection bias)

Allocation concealment Low risk

Low risk ction bias)
Quote: "The university's investigational pharmacy randomized all patients (in block sizes of eight using computer generated randomization with no clinical information) to either inositol or placebo in a 1:1 manner."
Pharmacy-controlled allocation. Efforts made to conceal placebo identity. Quote: "All eligible study patients were started on inositol $6 \mathrm{~g} /$ day or matching placebo powder for 2 weeks. At week 2 , the dose was increased to $12 \mathrm{~g} /$ day (or the same amount of matching powder) for 2 weeks, and then $18 \mathrm{~g} /$ day or matching amount of placebo powder for the rest of the study. Participants were given identical measuring spoons and instructed to take the required dose divided in three increments to be taken at each meal (e.g. starting with 2 g three times a day)".

\begin{tabular}{ll}
\hline $\begin{array}{l}\text { Blinding (performance } \\
\text { bias and detection bias) }\end{array} \quad$ Low risk & $\begin{array}{l}\text { Participants were blinded throughout the study period as per the study proto- } \\
\text { col on ClinicalTrials.gov. }\end{array}$
\end{tabular}

Self-reported outcomes

$\begin{array}{ll}\begin{array}{l}\text { Blinding (performance } \\ \text { bias and detection bias) }\end{array} \quad \text { Low risk } & \begin{array}{l}\text { Outcomes assessors reported as blinded throughout the study period as per } \\ \text { the study protocol on ClinicalTrials.gov. }\end{array}\end{array}$

Objective outcomes

Incomplete outcome data Low risk

(attrition bias)

Self-reported outcomes
Quote: "More participants withdrew from the placebo group (7/19; 37\%) compared to the inositol $(0 / 19 ; 0 \%)$ group. Reasons for withdrawal include time commitment was too great; and lost to follow-up."

No information was provided on sample characteristics at endpoint; however, demographics and clinical characteristics of the participants at baseline are presented. There were also no statistically significant imbalances in demographics or baseline trichotillomania symptoms between treatment groups.

Intention-to-treat analysis was employed: Quote: "Of the 38 patients enrolled, $30(76.3 \%)$ had complete data and two (5.3\%) only had baseline data. Roughly $5 \%$ (two participants) dropped out/had missing data at each subsequent visit for a total of seven (18.4\%) dropouts over the course of the study. There was one patient missing visit 5 who returned to complete visit 6 , whereas other dropouts did not return to the study (Fig. 1). Notably, all of the dropouts were from the placebo group". No significant differences were found between completers and non-completers except for a difference in education levels.

\begin{tabular}{lll}
\hline $\begin{array}{l}\text { Incomplete outcome data } \\
\text { (attrition bias) } \\
\text { Objective outcomes }\end{array}$ & Low risk & See above \\
\hline $\begin{array}{l}\text { Selective reporting (re- } \\
\text { porting bias) }\end{array}$ & Low risk & $\begin{array}{l}\text { All outcomes in protocol reported on and additional outcomes added in the } \\
\text { paper. Additional outcomes did not influence the conclusions drawn. }\end{array}$ \\
\hline Other bias & Unclear risk & $\begin{array}{l}\text { More females than males, concomitant antidepressants. Mainly white partici- } \\
\text { pants. No significant differences between groups. Authors worked for pharma- } \\
\text { ceutical companies as consultants. }\end{array}$ \\
\hline
\end{tabular}

Ninan 2000

\section{Study characteristics}


Ninan 2000 (Continued)

\begin{tabular}{|c|c|}
\hline Methods & Randomised controlled parallel-arm trial, 9 weeks \\
\hline Participants & $\begin{array}{l}\text { SAMPLE } \\
23 \text { DSM-III-R TTM, } 10 \text { participants in clomipramine arm/7 in CBT arm/6 in placebo arm; mean age } 33.38 \\
\text { (22 to 53) years; } 13 \text { women/3 men; mean duration of hair-pulling: } 20.62 \text { (10 to } 40 \text { ) years } \\
\text { SCREENING } \\
\text { Primary diagnosis: SCID-R } \\
\text { Comorbidities: no information given }\end{array}$ \\
\hline Interventions & Description: clomipramine (50 to $250 \mathrm{mg} / \mathrm{d}$ ) versus placebo versus HRT \\
\hline Outcomes & $\begin{array}{l}\text { Primary outcomes: NIMH-TSS, NIMH-TIS, CGI-I } \\
\text { Secondary outcomes: BDI, STAI }\end{array}$ \\
\hline Notes & $\begin{array}{l}\text { INDUSTRY SUPPORT } \\
\text { Industry-funded: unknown } \\
\text { Medication provided by industry: yes } \\
\text { Any of the authors work for industry: unknown }\end{array}$ \\
\hline
\end{tabular}

\section{Risk of bias}

\begin{tabular}{|c|c|c|}
\hline Bias & Authors' judgement & Support for judgement \\
\hline $\begin{array}{l}\text { Random sequence genera- } \\
\text { tion (selection bias) }\end{array}$ & Unclear risk & Method of randomisation was not disclosed \\
\hline $\begin{array}{l}\text { Allocation concealment } \\
\text { (selection bias) }\end{array}$ & Unclear risk & Method of allocation concealment was not disclosed \\
\hline $\begin{array}{l}\text { Blinding (performance } \\
\text { bias and detection bias) } \\
\text { Self-reported outcomes }\end{array}$ & Unclear risk & Method of blinding was not disclosed \\
\hline $\begin{array}{l}\text { Blinding (performance } \\
\text { bias and detection bias) } \\
\text { Objective outcomes }\end{array}$ & Low risk & $\begin{array}{l}\text { Pre- and post-treatment assessments were performed by an independent as- } \\
\text { sessor who was blinded to treatment condition. }\end{array}$ \\
\hline $\begin{array}{l}\text { Incomplete outcome data } \\
\text { (attrition bias) } \\
\text { Self-reported outcomes }\end{array}$ & High risk & $\begin{array}{l}23 \text { participants were randomly assigned ( } 10 \text { to clomipramine, } 6 \text { to placebo and } \\
7 \text { to CBT), but results from only } 16 \text { participants were analysed. Attrition rate of } \\
4 / 10=40 \% \text { for clomipramine ( } 3 \text { because of significant side effects) and } 1 / 6= \\
17 \% \text { for placebo }\end{array}$ \\
\hline $\begin{array}{l}\text { Incomplete outcome data } \\
\text { (attrition bias) } \\
\text { Objective outcomes }\end{array}$ & Unclear risk & As above \\
\hline $\begin{array}{l}\text { Selective reporting (re- } \\
\text { porting bias) }\end{array}$ & Unclear risk & $\begin{array}{l}\text { Insufficient information to permit judgement (trial protocol not obtained). Re- } \\
\text { sults for reduction of depression symptoms not reported. }\end{array}$ \\
\hline Other bias & Unclear risk & Medication provided by industry. Mostly female participants. \\
\hline
\end{tabular}




\section{Study characteristics}

\begin{tabular}{|c|c|}
\hline Methods & Randomised controlled cross-over trial, 12 weeks with 5-week washout period \\
\hline \multirow[t]{5}{*}{ Participants } & SAMPLE \\
\hline & $\begin{array}{l}23 \text { participants with compulsive, repetitive hair-pulling for at least the past } 12 \text { months; mean age } 39 \text { ( } 21 \\
\text { to } 68 \text { ) years; } 14 \text { females/ } 2 \text { males; mean age at onset: } 21 \text { years; duration of hair-pulling } 2 \text { to } 40 \text { years; co- } \\
\text { morbidities: anxiety disorders in 12/16 participants }\end{array}$ \\
\hline & SCREENING \\
\hline & Primary diagnosis: MTAI \\
\hline & Comorbidities: MTAI (contains screening questions for DSM-III-R axis I disorders) \\
\hline Interventions & Description: fluoxetine ( 20 to $80 \mathrm{mg} / \mathrm{d}$ ) versus placebo $\times 12$ weeks \\
\hline \multirow[t]{6}{*}{ Outcomes } & Primary outcomes: \\
\hline & 1. Number of hair-pulling episodes/week \\
\hline & 2. Estimated number of hairs pulled/week \\
\hline & 3. Rating of severity of the urge to pull hair/week \\
\hline & 4. Rating of severity of hair-pulling/week \\
\hline & Secondary outcomes: HAM-D, BDI. \\
\hline \multirow[t]{4}{*}{ Notes } & INDUSTRY SUPPORT \\
\hline & Industry-funded: yes \\
\hline & Medication provided by industry: yes \\
\hline & Any of the authors work for industry: not mentioned \\
\hline
\end{tabular}

\section{Risk of bias}

\begin{tabular}{|c|c|c|}
\hline Bias & Authors' judgement & Support for judgement \\
\hline $\begin{array}{l}\text { Random sequence genera- } \\
\text { tion (selection bias) }\end{array}$ & Low risk & Computer-generated random number assignment \\
\hline $\begin{array}{l}\text { Allocation concealment } \\
\text { (selection bias) }\end{array}$ & Low risk & Central allocation (from correspondence with the first author) \\
\hline $\begin{array}{l}\text { Blinding (performance } \\
\text { bias and detection bias) } \\
\text { Self-reported outcomes }\end{array}$ & Low risk & $\begin{array}{l}\text { Participants were blinded throughout the study (from correspondence with } \\
\text { the first author) }\end{array}$ \\
\hline $\begin{array}{l}\text { Blinding (performance } \\
\text { bias and detection bias) } \\
\text { Objective outcomes }\end{array}$ & Low risk & $\begin{array}{l}\text { All study personnel including outcome assessors were blinded throughout the } \\
\text { study (from correspondence with the first author) }\end{array}$ \\
\hline $\begin{array}{l}\text { Incomplete outcome data } \\
\text { (attrition bias) } \\
\text { Self-reported outcomes }\end{array}$ & High risk & $\begin{array}{l}\text { Intention-to-treat analysis was not used: only the } 16 \text { participants who com- } \\
\text { pleted the study were included in the analysis and the } 7 \text { dropouts were exclud- } \\
\text { ed from the analysis (from correspondence with the first author). Fluoxetine }\end{array}$ \\
\hline
\end{tabular}




\begin{tabular}{lll}
\hline $\begin{array}{l}\text { Incomplete outcome data } \\
\text { (attrition bias) } \\
\text { Objective outcomes }\end{array}$ & Unclear risk & As above \\
\hline $\begin{array}{l}\text { Selective reporting (re- } \\
\text { porting bias) }\end{array}$ & Unclear risk & Insufficient information to permit judgement (trial protocol not obtained) \\
\hline Other bias & Unclear risk & $\begin{array}{l}\text { Industry-funded; medications provided by industry. Mostly female partici- } \\
\text { pants. }\end{array}$ \\
\hline
\end{tabular}

Swedo 1989

\section{Study characteristics}

\begin{tabular}{|c|c|c|}
\hline Methods & \multicolumn{2}{|c|}{ Randomised cross-over head-to-head trial, 5 weeks with no washout period } \\
\hline \multirow[t]{5}{*}{ Participants } & \multicolumn{2}{|l|}{ SAMPLE } \\
\hline & \multicolumn{2}{|c|}{$\begin{array}{l}13 \text { participants with repetitive, compulsive hair-pulling resulting in observable hair loss; mean age } 31.6 \\
\text { (16 to } 44 \text { ) years; all women; mean age of onset } 13.8 \text { ( } 4 \text { to } 32 \text { ) years; mean duration of diagnosis } 16.2 \text { ( } 4 \text { to } \\
\text { 33) years; comorbidities: } 6 \text { participants had history of depressive episodes, } 4 \text { significant drug/alcohol } \\
\text { use, } 5 \text { history of generalised anxiety disorders, } 1 \text { panic disorder }\end{array}$} \\
\hline & \multicolumn{2}{|l|}{ SCREENING } \\
\hline & \multicolumn{2}{|c|}{ Primary diagnosis: semi-structured psychiatric interviews } \\
\hline & \multicolumn{2}{|c|}{$\begin{array}{l}\text { Comorbidities: Leyton Obsessional Inventory, Schedule for Affective Disorders and Schizophrenia, } \\
\text { Structured interview for assessment of personality disorders }\end{array}$} \\
\hline Interventions & \multicolumn{2}{|c|}{$\begin{array}{l}\text { Description: clomipramine ( } 50 \text { to } 250 \mathrm{mg} / \mathrm{d} \text { ) versus desipramine }(50 \text { to } 250 \mathrm{mg} / \mathrm{d}) \times 5 \text { weeks (capsules of } \\
\text { clomipramine and desipramine were identical) }\end{array}$} \\
\hline \multirow[t]{3}{*}{ Outcomes } & \multirow{3}{*}{\multicolumn{2}{|c|}{$\begin{array}{l}\text { Primary outcomes: NIMH-TSS, NIMH-TIS, Physician-Rated Clinical Progress (maximum score 20) } \\
\text { Secondary outcomes: NIMH Global Assessment Scales for depression and anxiety } \\
\text { Side effects: standardised checklist of } 23 \text { physical symptoms, rated } 0=\text { none, } 1=\text { slight and } 2=\text { severe }\end{array}$}} \\
\hline & & \\
\hline & & \\
\hline \multirow[t]{4}{*}{ Notes } & \multicolumn{2}{|l|}{ INDUSTRY SUPPORT } \\
\hline & \multicolumn{2}{|c|}{ Industry-funded: no (from correspondence with the first author) } \\
\hline & \multicolumn{2}{|c|}{ Medication provided by industry: no (from correspondence with the first author) } \\
\hline & \multicolumn{2}{|c|}{ Any of the authors work for industry: unknown } \\
\hline \multicolumn{3}{|l|}{ Risk of bias } \\
\hline Bias & Authors' judgement & Support for judgement \\
\hline $\begin{array}{l}\text { Random sequence genera- } \\
\text { tion (selection bias) }\end{array}$ & Low risk & Random-number table \\
\hline
\end{tabular}


Swedo 1989 (Continued)

Allocation concealment $\quad$ Low risk
(selection bias)

\begin{tabular}{|c|c|c|}
\hline $\begin{array}{l}\text { Blinding (performance } \\
\text { bias and detection bias) }\end{array}$ & Unclear risk & $\begin{array}{l}\text { Participants were blinded throughout the study (from correspondence with } \\
\text { first author) }\end{array}$ \\
\hline
\end{tabular}

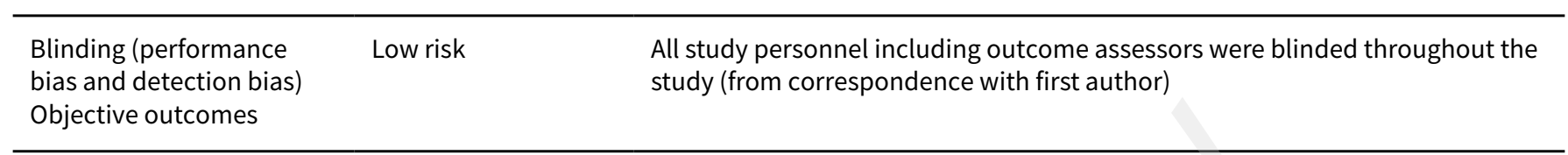

$\begin{array}{ll}\text { Incomplete outcome data } \quad \text { Low risk } & \text { Results from all participants who were randomly assigned were analysed (in- } \\ \text { (attrition bias) } & \text { tention-to-treat analysis used). One participant excluded from randomisation } \\ \text { Self-reported outcomes } & \text { step due to }>20 \% \text { improvement during initial placebo phase. }\end{array}$

\begin{tabular}{lll}
\hline $\begin{array}{l}\text { Incomplete outcome data } \\
\begin{array}{l}\text { (attrition bias) } \\
\text { Objective outcomes }\end{array}\end{array}$ & $\begin{array}{l}\text { Lesults from all participants who were randomly assigned were analysed (in- } \\
\text { tention-to-treat analysis used). One participant excluded from randomisation } \\
\text { step due to }>20 \% \text { improvement during initial placebo phase. }\end{array}$ \\
\hline $\begin{array}{l}\text { Selective reporting (re- } \\
\text { porting bias) }\end{array}$ & Unclear risk & Insufficient information to permit judgement \\
\hline \begin{tabular}{l} 
Other bias \\
\hline
\end{tabular} & Unclear risk & All female participants \\
\hline
\end{tabular}

Van Ameringen 2010

\section{Study characteristics}

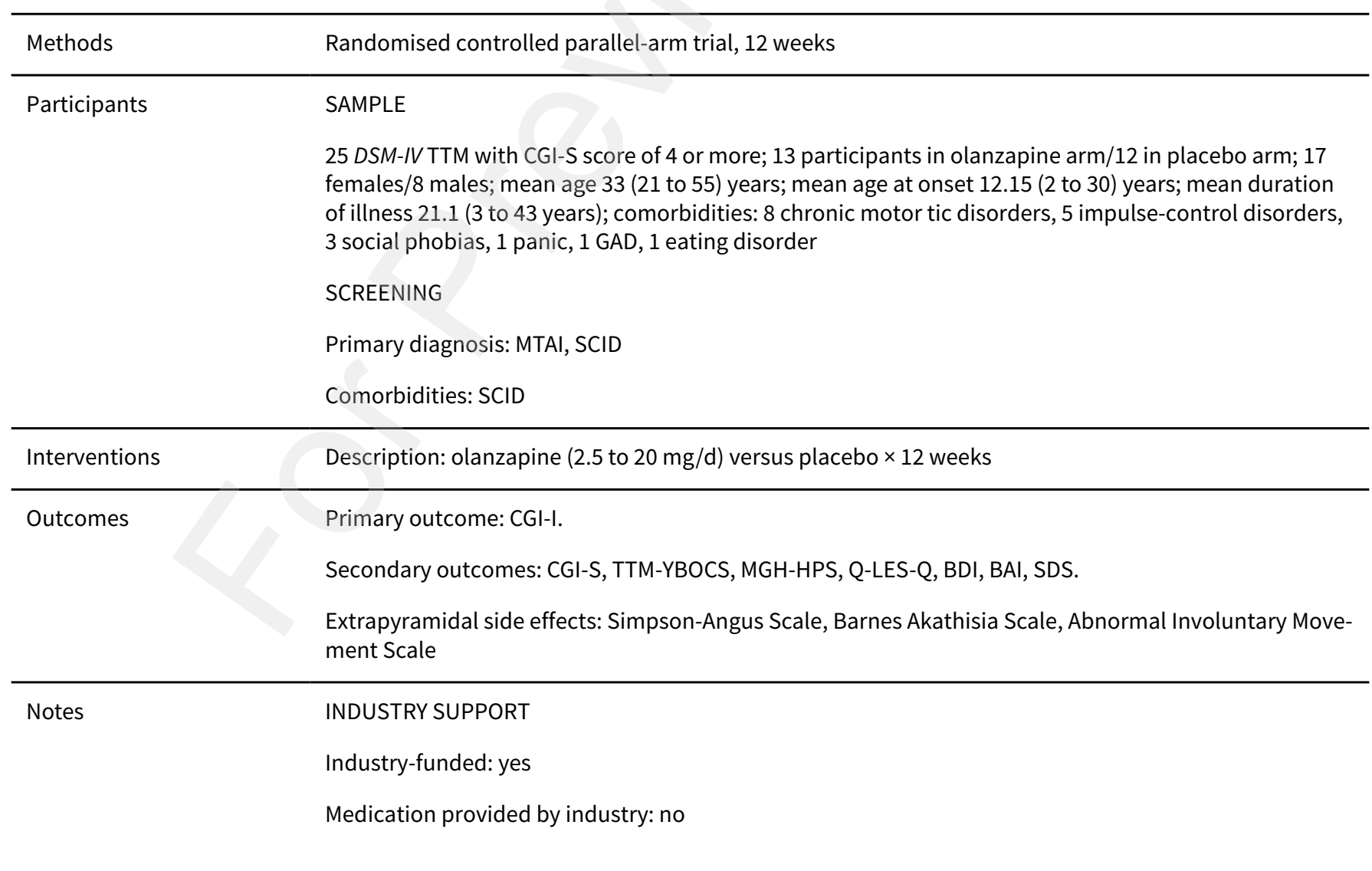


Van Ameringen 2010 (Continued)

Any of the authors work for industry: no

\section{Risk of bias}

\begin{tabular}{|c|c|c|}
\hline Bias & Authors' judgement & Support for judgement \\
\hline $\begin{array}{l}\text { Random sequence genera- } \\
\text { tion (selection bias) }\end{array}$ & Low risk & $\begin{array}{l}\text { Random allocation sequence was implemented using a central telephone, } \\
\text { generated by a statistician and sent to a central pharmacy }\end{array}$ \\
\hline $\begin{array}{l}\text { Allocation concealment } \\
\text { (selection bias) }\end{array}$ & Low risk & Pharmacy-controlled allocation \\
\hline $\begin{array}{l}\text { Blinding (performance } \\
\text { bias and detection bias) } \\
\text { Self-reported outcomes }\end{array}$ & Low risk & $\begin{array}{l}\text { Participants were blinded throughout the study (from correspondence with } \\
\text { first author) }\end{array}$ \\
\hline $\begin{array}{l}\text { Blinding (performance } \\
\text { bias and detection bias) } \\
\text { Objective outcomes }\end{array}$ & Low risk & $\begin{array}{l}\text { All study personnel including outcome assessors were blinded throughout the } \\
\text { study (from correspondence with first author) }\end{array}$ \\
\hline $\begin{array}{l}\text { Incomplete outcome data } \\
\text { (attrition bias) } \\
\text { Self-reported outcomes }\end{array}$ & Low risk & $\begin{array}{l}\text { Intention-to-treat analysis was used (all participants who were randomly as- } \\
\text { signed were included in data analysis) }\end{array}$ \\
\hline $\begin{array}{l}\text { Incomplete outcome data } \\
\text { (attrition bias) } \\
\text { Objective outcomes }\end{array}$ & Low risk & $\begin{array}{l}\text { Intention-to-treat analysis was used (all participants who were randomly as- } \\
\text { signed were included in data analysis) }\end{array}$ \\
\hline $\begin{array}{l}\text { Selective reporting (re- } \\
\text { porting bias) }\end{array}$ & Low risk & $\begin{array}{l}\text { Trial protocol showed no additional outcomes unreported in the publication. } \\
\text { Data for the following self-reported secondary outcome measures were not } \\
\text { included in the paper: Q-LES-Q, BDI, BAI and SDS (from correspondence with } \\
\text { first author-no significant differences between treatment and placebo for } \\
\text { these outcomes). }\end{array}$ \\
\hline Other bias & Unclear risk & Industry-funded, female predominance of participants \\
\hline
\end{tabular}

ADHD: attention-deficit hyperactivity disorder; BAI: Beck Anxiety Inventory; BDI: Beck Depression Inventory; CBT: cognitive-behavioural therapy; CDI: Children's Depression Inventory; CGI-I: Clinical Global Impression - Improvement; CGI-S: Clinical Global Impression - Severity; DIS: Diagnostic Interview Schedule; GAD: generalised anxiety disorder; HAM-A: Hamilton Anxiety Rating Scale; HAM-D: Hamilton Depression Rating Scale; HRT: habit-reversal therapy; MASC: Multidimensional Anxiety Scale for Children; MDD: major depressive disorder; MGH-HPS: Massachusetts General Hospital Hair-Pulling Scale; MINI: Mini-International Neuropsychiatric Interview; MTAI: Minnesota Trichotillomania Assessment Inventory; NAC: N-acetylcysteine; NIMH-TIS: NIMH Trichotillomania Impairment Scale; NIMH-TSS: National Institute of Mental Health-Trichotillomania Severity Scale; OCD: obsessive-compulsive disorder; PITS: Psychiatric Institute Trichotillomania Scale; Q-LES-Q: Quality of Life Enjoyment and Satisfaction Questionnaire; QoLI: Quality of Life Inventory; SCID: Structured Clinical Interview for DSM-IV Disorders; SCID-R: Structured Clinical Interview for DSM-III-R Disorders (revised); SDS: Sheehan Disability Scale; SST: Stop Signal Task; STAI: State-Trait Anxiety Inventory; TSC-C: Trichotillomania Scale for Children-Child version; TSC-P: Trichotillomania Scale for Children-Parent versions; TTMIS: Trichotillomania Impact Scale; TTM-YBOCS: Yale-Brown Obsessive-Compulsive Scale for TTM

\section{Characteristics of excluded studies [ordered by year]}

\begin{tabular}{ll}
\hline Study & Reason for exclusion \\
\hline Black 1992 & Case report (i.e. not a randomised controlled trial). \\
\hline Riley 1993 & Double-blind reversal design (i.e. not a randomised controlled trial); paediatric population. \\
\hline
\end{tabular}




\begin{tabular}{ll}
\hline Study & Reason for exclusion \\
\hline Stein 1995 & $\begin{array}{l}\text { Outcome measured was neuroendocrine (serotonergic response), not severity of TTM symptoms. } \\
\text { Not a pharmacotherapy RCT. }\end{array}$ \\
\hline Epperson 1996 & Only two participants. \\
\hline Stein 1997 & $\begin{array}{l}\text { Outcome measured was acute behavioural response, not reduction of TTM symptoms over time or } \\
\text { efficacy of intervention. Not a pharmacotherapy RCT. }\end{array}$ \\
\hline Ninan 1998 & Report is only for open-label phase I trial (i.e. not a randomised controlled trial). \\
\hline Van Minnen 2003 & Waiting-list control group (i.e. no placebo group) \\
\hline Chamberlain 2010 & $\begin{array}{l}\text { Outcome measured was neurocognitive performance (motor inhibitory control), not severity of } \\
\text { TTM symptoms. Not a pharmacotherapy RCT. }\end{array}$ \\
\hline Dufour 2010 & Dietary intervention, not pharmacotherapy. \\
\hline Golubchik 2011 & Open-label design. \\
\hline
\end{tabular}

Characteristics of ongoing studies [ordered by study ID]

IRCT20181009041282N2

Study name
Compare the Effectiveness of Group Therapy Combination of Acceptance and Commitment Therapy and Habit Reversal Training to Drug Therapy in Reducing Symptoms, Anxiety and Depression of Patients with Trichotillomania (IRCT20181009041282N2)

Target sample size: 30

Age: from 18 to 50 years old

Gender: both

Inclusion criteria: clinical and psychiatric diagnosis of trichotillomania, anxiety and depression. Obtaining a minimum score in the MGH-HPS, BAI, and BDI. Aged 18 to 50 years old. Having at least an elementary education. Discontinue psycho-drug treatment at least one month before entering the plan. Participants do not suffer from psychotic diseases that interfere with this research.

Exclusion criteria: unwillingness to cooperate in research. People with other mental and neurological diseases. People who receive different medical or psychological treatments.

\section{Interventions \\ Intervention group: the first intervention group participates in 10 sessions, and each session is 100 minutes of group therapy, combination of habit-reversal training and acceptance and commitment treatment. ACT encourages a person to accept what is beyond his control and commitment to so- cial goals. In fact, ACT uses acceptance processes, mindfulness, and change in behavior and com- mitment to create psychological flexibility. \\ Intervention group: the second group receives citalopram with a baseline of $40 \mathrm{mg}$ per day, reach- ing $60 \mathrm{mg}$ per day after 5 weeks. In general, this medication will last for 8 weeks. \\ Control group: the control group receives only 5 sessions and each session is 40 minutes of habit- reversal training.}


IRCT20181009041282N2 (Continued)

Secondary outcomes: Not stated

\begin{tabular}{ll}
\hline Starting date & 16 November 2018 (Gregorian calendar), 1397/08/25 (Iranian calendar) \\
\hline Contact information & $\begin{array}{l}\text { Samaneh Eshaghzadeh, Torbate-Heidaria University of Medical Sciences, Razi street, Torbat Hey- } \\
\text { darieh, Razavi Khorasan, } 9516915169\end{array}$ \\
& Phone: +985152226011 \\
Email: eshaghzadeh0@gmail.com
\end{tabular}

Notes

Name of recruitment centre: Psychology Clinic and Student Counseling Center of Ferdowsi University of Mashhad

Dates reported as Gregorian calendar date, Iranian calendar date.

Expected recruitment end date

20 March 2019, 1397/12/29

Actual recruitment start date

22 October 2018, 1397/07/30

Actual recruitment end date

20 January 2019, 1397/10/30

Trial completion date

20 January 2019, 1397/10/30

IRCT registration number: IRCT20181009041282N2

Registration date: 16 November 2018, 1397/08/25

Registration timing: registered_while_recruiting

Study name Glutathione Levels and Compulsivity (NCT02794389)

Methods A randomised, double-blind, placebo-controlled, interventional, cross-over assignment study Masking: quadruple (participant, care provider, investigator, outcomes assessor)

Sexes eligible for study: female

Accepts healthy volunteers: yes

Inclusion criteria: participant is willing and able to give informed consent for participation in the study; females aged 18 to 40 years; score between 9 and 19 on the EAT-26; participant is a fluent English speaker.

Exclusion criteria: any current psychotropic medications; any other significant disease or disorder which, in the opinion of the investigator, may either put the participant at risk because of participation in the trial, or may influence the result of the trial, or the participant's ability to participate in the study; body mass index (BMI) below 18.5, indicating that they are underweight; any Axis I psy- 
NCT02794389 (Continued)

chiatric disorder; history of allergies to drugs or vaccines or any component of the NAC or placebo capsule (gelatine, magnesium stearate, NAC); pregnant, could be pregnant, breast feeding, or high risk of pregnancy (no reliable contraception use but sexual activity); has taken part in a psychological or medical experiment involving taking any kinds of drugs within the last 6 weeks; has had recent surgery; taking selenium, vitamin E or NAC; current regular cigarette smoking of over 5 cigarettes per day.

Interventions

Experimental: $\mathrm{N}$-acetylcysteine, $1200 \mathrm{mg}$ for 2 days, $2400 \mathrm{mg}$ for 7 days

Placebo comparator: placebo, magnesium stearate capsules

\begin{tabular}{|c|c|}
\hline \multirow[t]{3}{*}{ Outcomes } & Primary outcome measure: Berg Card-Sort Task \\
\hline & $\begin{array}{l}\text { Secondary outcome measures: attention switching task, affective go/no-go task, Cambridge gam- } \\
\text { bling task, sequential learning task, delay discounting task, HADS, EAT-26, FERT, side effects, per- } \\
\text { centage of participants who correctly guess which treatment group they are in. }\end{array}$ \\
\hline & Time frame: after 9 days of taking $\mathrm{N}$-acetylcysteine and after 9 days of taking placebo. \\
\hline \multirow[t]{3}{*}{ Starting date } & Study start date: March 2016 \\
\hline & Actual primary completion date: April 2017 \\
\hline & Actual study completion date: April 2017 \\
\hline Contact information & Principal investigator: Phil J Cowen, Prof. University of Oxford \\
\hline \multirow[t]{4}{*}{ Notes } & $\begin{array}{l}\text { Locations: United Kingdom, Department of Psychiatry, University of Oxford. Oxford, Oxfordshire, } \\
\text { United Kingdom, OX3 7JX }\end{array}$ \\
\hline & Sponsors and collaborators: University of Oxford \\
\hline & ClinicalTrials.gov Identifier: NCT02794389 \\
\hline & Last update posted: 7 November 2018 \\
\hline
\end{tabular}

\section{NCT03530800}

Study name Dronabinol in Trichotillomania and Other Body-Focused Repetitive Behaviors (NCT03530800)

Methods A double-blind, placebo-controlled, interventional, parallel assignment study

Masking: triple (participant, investigator, outcomes assessor)

Participants

Estimated enrolment: 50 participants

Ages eligible for study: 18 to 75 years (adult, older adult)

Sexes eligible for study: all

Accepts healthy volunteers: no

Inclusion criteria: current DSM-5 trichotillomania; ability to understand and sign the consent form.

Exclusion criteria: unstable medical illness based on history of clinically significant abnormalities on baseline physical examination; current pregnancy or lactation, or inadequate contraception in women of childbearing potential; subjects considered an immediate suicide risk based on the Columbia Suicide Severity Rating Scale (C-SSRS) (www.cssrs.columbia.edu/docs); past 12-month DSM-5 psychiatric disorder other than trichotillomania; illegal substance use based on urine toxicology screening; use of any other psychotropic medication (except a hypnotic pro re nata i.e. 
NCT03530800 (Continued)

when required); previous treatment with dronabinol; cognitive impairment that interferes with the capacity to understand and self administer medication or provide written informed consent.

Interventions

Active comparator: dronabinol. Participants will receive dronabinol $5 \mathrm{mg}$ once daily for two weeks, $5 \mathrm{mg}$ twice daily for the subsequent two weeks, and $5 \mathrm{mg}$ three times daily for the final six weeks. Dose escalations will only be done if the investigator deems necessary.

Placebo comparator: placebo. Participants will receive placebo for 10 weeks.

\section{Outcomes}

Primary outcome measures: NIMH-TSS, SP-SAS

Time frame: up to 10 weeks
Actual study start date: 1 October 2018

Estimated primary completion date: 1 June 2020

Estimated study completion date: 1 August 2020

Stephanie Valle, BA 773-834-3778 svalle@yoda.bsd.uchicago.edu

Elizabeth Cavic, BS 773-702-9066 ec642@bsd.uchicago.edu

\section{Notes}

Sponsors and collaborators: University of Chicago

Location: University of Chicago

Principal investigator: Jon E Grant, JD, MD, MPH, University of Chicago

ClinicalTrials.gov Identifier: NCT03530800

Last update posted: 21 July 2021

NCT03797521

Study name $\quad$ A Study in Patients With Trichotillomania (TTM) (NCT03797521)

\section{Methods}

A phase 2 interventional, randomised, double-blind, placebo-controlled, parallel assignment study

Masking: quadruple (participant, care provider, investigator, outcomes assessor)

\section{Participants}

Estimated enrolment: 120 participants

Ages eligible for study: 18 to 45 years

Sexes eligible for study: all

Accepts healthy volunteers: no

Inclusion criteria: (1) adult, female or male, 18 to 45 years of age, inclusive, at screening, (2) provided signed written informed consent with willingness and ability to comply with all aspects of the protocol, (3) diagnosis of current TTM based on Diagnostic and Statistical Manual of Mental Disorders, fifth edition (DSM-5) criteria and confirmed using the clinician-administered Mini International Neuropsychiatric Interview (MINI).

In addition, subjects should: (1) have a history of TTM for at least one year, (2) have a history of daily hair-pulling for at least 6 months prior to the first dose, (3) except for SSRIs or SNRIs, not have used any psychoactive medications including, but not limited to, other antidepressants, anxiolytics, mood stabilizers, anti-psychotics, benzodiazepines, stimulants, sulfasalazine, and St. John's Wort 30 days prior to first dose. Subjects will be allowed to maintain background therapy with 
SSRIs or SNRIs if on stable regimen for a minimum of 90 days prior to first dose and there are no anticipated changes to the SSRI/SNRI during course of trial, (4) not have used N-acetylcysteine for at least 90 days prior to the first dose, (5) not have used gemfibrozil or repaglinide for 1 week prior to the first screening visit, (6) medically healthy with no clinically significant findings in medical history, physical examination, laboratory profiles, vital signs, or ECG, as deemed by the Principal Investigator (PI) or designee. (7) For a female of childbearing potential: either be sexually inactive (abstinent as a life style) for 28 days prior to the first dosing and throughout the study or be using one of the following acceptable birth control options: oral contraception for at least 3 months prior to the first dosing along with either a physical (e.g. condom, diaphragm) or a chemical (e.g. spermicide) barrier method from the time of screening and throughout the study; IUD (either hormone-releasing or non-hormone releasing) for at least minimum duration per current labelling along with either a physical (e.g. condom, diaphragm) or a chemical (e.g. spermicide) barrier method from the time of screening and throughout the study; depo contraception for at least minimum duration per current labelling prior to the first dosing along with either a physical (e.g. condom, diaphragm) or a chemical (e.g. spermicide) barrier method from the time of screening and throughout the study; double physical barrier method (e.g. condom and diaphragm) from 14 days prior to the first dose and throughout the study; physical plus chemical barrier method (e.g. condom with spermicide) from 14 days prior to the first dose and throughout the study. In addition, female subjects of childbearing potential will be advised to remain sexually inactive or to keep the same birth control method for at least 30 days following the last dose. (8) Females of non-childbearing potential: must have undergone one of the following sterilisation procedures, at least 6 months prior to the first dose: hysteroscopic sterilisation; bilateral tubal ligation or bilateral salpingectomy; hysterectomy; bilateral oophorectomy; or be postmenopausal with amenorrhoea for at least 1 year prior to the first dose with serum follicle stimulating hormone levels consistent with postmenopausal status or have medically documented history of biological or congenital sterility; (9) A non-vasectomized male subject must agree to use a condom with spermicide or abstain from sexual intercourse during the study until 30 days beyond the last dose of study drug/placebo. (10) If male, must agree not to donate sperm from the first dose until 30 days after the last dose administration. (11) Participants must be able to fluently read and write in English. (13) Participants must understand the study procedures in the informed consent form (ICF) and be willing and able to comply with the protocol.

Exclusion criteria: (1) females who are pregnant or breastfeeding or intend to become pregnant during the study period or within 30 days of the final dose of study drug, (2) subjects engaged in cognitive behavioral therapy (CBT) for TTM or other body-focused repetitive behavior or any obsessive-compulsive related or impulse control disorder any time within 60 days prior to first dose. For other psychotherapies, subject must have been engaged in that psychotherapy for a minimum of 60 days at the time of first dose and must be willing to maintain the same frequency and type of therapy for the duration of the study period, (3) subjects engaged in any other behavioral interventions (e.g. wearable devices, behavioral self-help strategies) within 60 days prior to first dose, (4) mentally or legally incompetent, (5) suffered a concussion in the past 6 months prior to screening. Any history of traumatic brain injury with loss of consciousness in the year prior to first screening visit, (6) any lifetime history of any psychotic disorder, including schizophrenia or any bipolar or bipolar-related disorder as determined by clinical history or confirmed at screening with the MINI, version 7.0.2, (7) current major depressive episode confirmed at screening with the MINI, version 7.0 .2 , (8) according to PI judgment, the presence of any emotional problems or psychiatric disorders that may obscure evaluation of primary TTM or pose a risk to subject safety or stability during the study period. Other emotional problems or diagnoses may include, but are not limited to, other body-focused repetitive behaviours, post-traumatic stress disorder, obsessive-compulsive disorder, panic disorder, compulsive gambling, borderline personality disorder, or antisocial personality disorder, (9) history of any injury, illness, or condition that, in the opinion of the PI or designee, might confound the results of the study or pose an additional risk to the subject by their participation in the study, (10) laboratory evidence of renal impairment (e.g. a creatine clearance of < 80), (11) presence of any substance use disorder or, in the opinion of the PI or designee, problematic substance use (excluding nicotine or caffeine) within the 2 years prior to screening, (12) history of seizure disorder with the exception of subjects who have been off anti-seizure medication and have not had a seizure in the past 5 years, (13) subjects with any of the following: (a) any psychiatric hospitalisations in the past year; (b) imminent risk of suicide based on PI's or designee's clinical judgment or psychiatric examination; (c) active suicidal ideation in the past 6 months as evidenced by positive endorsement to Item 4 or 5 on the Columbia Suicide Severity Rating Scale (C-SSRS); OR (d) any history of suicidal behavior in the past year as evidenced by positive endorsement to any of 
NCT03797521 (Continued)

the suicidal behavior items on the C-SSRS, (14) has previously participated in any Promentis Phase 1 study, (15) participation in another interventional clinical study (including CBT or other behavioral intervention) within 30 days prior to the first screening visit. The 30 day window will be derived from the date of the last blood collection or dosing, whichever is later, in the previous study to the date of initiation of screening in the current study.

\begin{tabular}{|c|c|}
\hline \multirow[t]{4}{*}{ Interventions } & Experimental: SXC-2023 $50 \mathrm{mg}$ dosed once daily for 6 weeks \\
\hline & Experimental: SXC-2023 $200 \mathrm{mg}$ dosed once daily for 6 weeks \\
\hline & Experimental: SXC-2023 $800 \mathrm{mg}$ dosed once daily for 6 weeks \\
\hline & Placebo comparator: matching placebo dosed once daily for 6 weeks \\
\hline \multirow[t]{3}{*}{ Outcomes } & Primary outcome measure: treatment-emergent adverse events \\
\hline & Secondary outcome measures: MGH-HPS, CGI-S/C, PGI-S/C, TSD \\
\hline & Time frame: up to 7 weeks \\
\hline \multirow[t]{3}{*}{ Starting date } & Actual study start date: 19 December 2018 \\
\hline & Estimated primary completion date: 1 December 2019 \\
\hline & Estimated study completion date: 15 January 2020 \\
\hline Contact information & Study Director: Tricia Pimentel Cotter; Promentis Pharmaceuticals \\
\hline \multirow[t]{5}{*}{ Notes } & Responsible party: Promentis Pharmaceuticals, Inc. \\
\hline & ClinicalTrials.gov Identifier: NCT03797521 \\
\hline & Other study ID numbers: PRO-201 \\
\hline & First posted: 9 January 2019 \\
\hline & Last update posted: 2 December 2020 \\
\hline
\end{tabular}

ACT: Acceptance and Commitment Therapy; BAI: Beck Anxiety Inventory; BDI: Beck Depression Inventory; CGI-C: Clinical Global Impression - Change scale; CGI-I: Clinical Global Impressions - Improvement scale; CGI-S: Clinical Global Impressions - Severity scale; EAT-26: Eating Attitudes Test-26; ECG: electrocardiogram; FERT: Facial Expression Recognition Test; HADS: Hospital Anxiety and Depression Scale; IUD: intrauterine device; MGH-HPS: Massachusetts General Hospital Hair-Pulling Scale; MINI: Mini International Neuropsychiatric Interview; NIMH-TSS: National Institute of Mental Health-Trichotillomania Severity Scale; PGI-C: Patient Global Impressions - Change scale PGI-S: Patient Global Impressions - Severity scale; SP-SAS: Skin-Picking Symptom Assessment Scale; TTM: trichotillomania; TSD: Trichotillomania Symptom Diary

\section{DATA AND ANALYSES}

Comparison 1. Antioxidants versus placebo in adults

\begin{tabular}{lllll}
\hline Outcome or subgroup title & No. of studies & $\begin{array}{l}\text { No. of partici- } \\
\text { pants }\end{array}$ & Statistical method & Effect size \\
\hline 1.1 Treatment response & 1 & & $\begin{array}{l}\text { Risk Ratio (M-H, Random, } \\
95 \% \mathrm{Cl})\end{array}$ & Subtotals only \\
\hline
\end{tabular}




\begin{tabular}{|c|c|c|c|c|}
\hline Outcome or subgroup title & No. of studies & $\begin{array}{l}\text { No. of partici- } \\
\text { pants }\end{array}$ & Statistical method & Effect size \\
\hline $\begin{array}{l}\text { 1.1.1 Clinical Global Impressions - Im- } \\
\text { provement Scale (CGI-I) (no. of respon- } \\
\text { ders) }\end{array}$ & 1 & 36 & $\begin{array}{l}\text { Risk Ratio (M-H, Random, } \\
95 \% \mathrm{Cl})\end{array}$ & $2.25[0.84,5.99]$ \\
\hline $\begin{array}{l}1.2 \text { Reduction of TTM symptom severi- } \\
\text { ty }\end{array}$ & 1 & & $\begin{array}{l}\text { Mean Difference (IV, Random, } \\
95 \% \mathrm{CI})\end{array}$ & Subtotals only \\
\hline $\begin{array}{l}\text { 1.2.1 Clinical Global Impression - } \\
\text { Severity Scale (CGI-S) }\end{array}$ & 1 & 27 & $\begin{array}{l}\text { Mean Difference (IV, Random, } \\
95 \% \mathrm{CI})\end{array}$ & $-0.50[-1.31,0.31]$ \\
\hline $\begin{array}{l}\text { 1.2.2 Massachusetts General Hospital } \\
\text { Hairpulling Scale (MGH-HPS) }\end{array}$ & 1 & 27 & $\begin{array}{l}\text { Mean Difference (IV, Random, } \\
95 \% \mathrm{Cl})\end{array}$ & $-1.80[-6.22,2.62]$ \\
\hline $\begin{array}{l}\text { 1.2.3 NIMH Trichotillomania Severity } \\
\text { Scale (NIMH-TSS) }\end{array}$ & 1 & 27 & $\begin{array}{l}\text { Mean Difference (IV, Random, } \\
95 \% \mathrm{CI})\end{array}$ & $-1.00[-5.00,3.00]$ \\
\hline $\begin{array}{l}\text { 1.3 Reduction of comorbid symptoms } \\
\text { of depression }\end{array}$ & 1 & & $\begin{array}{l}\text { Mean Difference (IV, Random, } \\
95 \% \mathrm{Cl} \text { ) }\end{array}$ & Subtotals only \\
\hline $\begin{array}{l}\text { 1.3.1 Hamilton Depression Rating } \\
\text { Scale (HAM-D) }\end{array}$ & 1 & 27 & $\begin{array}{l}\text { Mean Difference (IV, Random, } \\
95 \% \mathrm{Cl})\end{array}$ & $0.60[-1.82,3.02]$ \\
\hline 1.4 Quality of Life - functional disability & 1 & & $\begin{array}{l}\text { Mean Difference (IV, Random, } \\
95 \% \mathrm{Cl} \text { ) }\end{array}$ & Subtotals only \\
\hline 1.4.1 Sheehan Disability Scale (SDS) & 1 & 27 & $\begin{array}{l}\text { Mean Difference (IV, Random, } \\
95 \% \mathrm{CI})\end{array}$ & $-0.60[-6.53,5.33]$ \\
\hline $\begin{array}{l}\text { 1.5 Tolerability of treatment - Dropouts } \\
\text { due to adverse events }\end{array}$ & 1 & & $\begin{array}{l}\text { Risk Ratio (M-H, Random, } \\
95 \% \mathrm{Cl})\end{array}$ & Subtotals only \\
\hline 1.5.1 Dropout rate & 1 & 28 & $\begin{array}{l}\text { Risk Ratio (M-H, Random, } \\
95 \% \mathrm{Cl} \text { ) }\end{array}$ & Not estimable \\
\hline
\end{tabular}

Analysis 1.1. Comparison 1: Antioxidants versus placebo in adults, Outcome 1: Treatment response

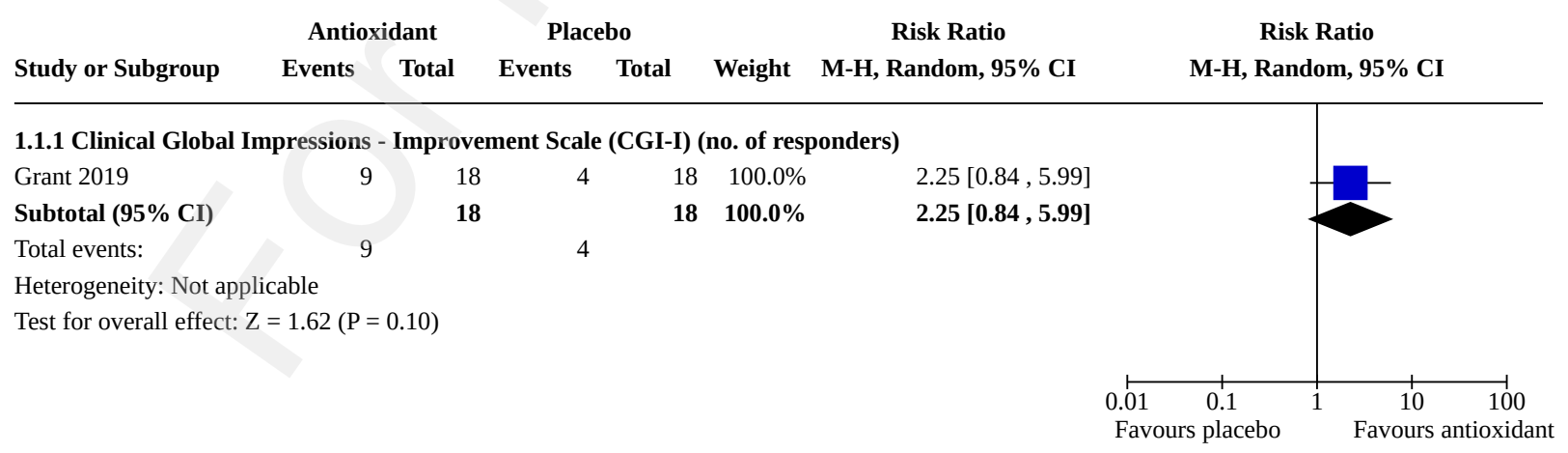


Analysis 1.2. Comparison 1: Antioxidants versus placebo in adults, Outcome 2: Reduction of TTM symptom severity

\begin{tabular}{|c|c|c|c|c|c|c|c|c|c|}
\hline & \multicolumn{3}{|c|}{ Antioxidant } & \multicolumn{3}{|c|}{ Placebo } & \multicolumn{2}{|r|}{ Mean Difference } & Mean Difference \\
\hline Study or Subgroup & Mean & SD & Total & Mean & SD & Total & Weight & IV, Random, 95\% CI & IV, Random, 95\% CI \\
\hline
\end{tabular}

1.2.1 Clinical Global Impression - Severity Scale (CGI-S)

$\begin{array}{llllllllll}\text { Grant } 2019 & 3.3 & 1.3 & 14 & 3.8 & 0.8 & 13 & 100.0 \% & -0.50[-1.31,0.31]\end{array}$

$\begin{array}{lllll}\text { Subtotal }(95 \% \mathrm{CI}) & 14 & 13 & \mathbf{1 0 0 . 0} \% & -0.50[-1.31,0.31]\end{array}$

Heterogeneity: Not applicable

Test for overall effect: $\mathrm{Z}=1.21(\mathrm{P}=0.23)$

1.2.2 Massachusetts General Hospital Hairpulling Scale (MGH-HPS)

$\begin{array}{llllllllll}\text { Grant } 2019 & 12.5 & 6.3 & 14 & 14.3 & 5.4 & 13 & 100.0 \% & -1.80[-6.22,2.62]\end{array}$

$\begin{array}{llll}14 & 13 & 100.0 \% & -1.80[-6.22,2.62]\end{array}$

Heterogeneity: Not applicable

Test for overall effect: $\mathrm{Z}=0.80(\mathrm{P}=0.42)$

1.2.3 NIMH Trichotillomania Severity Scale (NIMH-TSS)

$\begin{array}{lllllllll}\text { Grant } 2019 & 7.4 & 5.4 & 14 & 8.4 & 5.2 & 13 & 100.0 \% & -1.00[-5.00,3.00] \\ \text { Subtotal (95\% CI) } & & & \mathbf{1 4} & & & \mathbf{1 3} & \mathbf{1 0 0 . 0 \%} & \mathbf{- 1 . 0 0}[-\mathbf{5 . 0 0 , 3 . 0 0 ]}\end{array}$

Heterogeneity: Not applicable

Test for overall effect: $\mathrm{Z}=0.49(\mathrm{P}=0.62)$

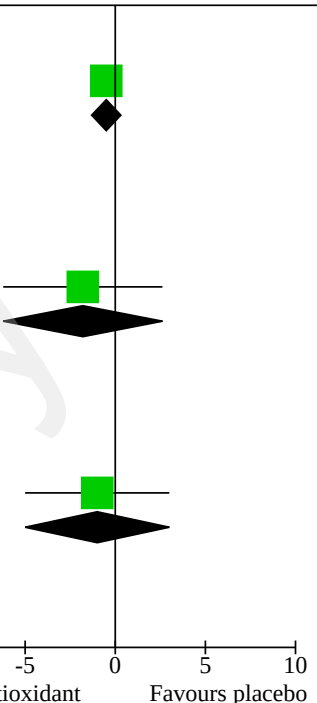

Analysis 1.3. Comparison 1: Antioxidants versus placebo in adults,

Outcome 3: Reduction of comorbid symptoms of depression

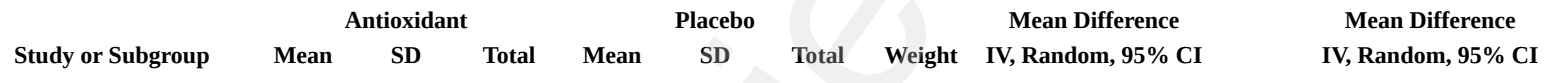

1.3.1 Hamilton Depression Rating Scale (HAM-D)

Grant 2019

Subtotal (95\% CI) 14

$0.60[-1.82,3.02]$

Heterogeneity: Not applicable

$13 \quad 100.0 \%$

$0.60[-1.82,3.02]$

Test for overall effect: $\mathrm{Z}=0.49(\mathrm{P}=0.63)$

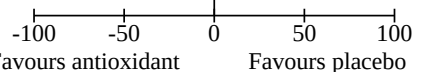

Analysis 1.4. Comparison 1: Antioxidants versus placebo in adults, Outcome 4: Quality of Life - functional disability

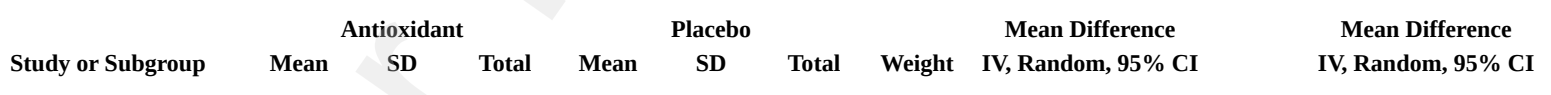

1.4.1 Sheehan Disability Scale (SDS)

$\begin{array}{lllllllll}\text { Grant } 2019 & 5.8 & 8.4 & 14 & 6.4 & 7.3 & 13 & 100.0 \% & -0.60[-6.53,5.33] \\ \text { Subtotal (95\% CI) } & & & \mathbf{1 4} & & & \mathbf{1 3} & \mathbf{1 0 0 . 0 \%} & \mathbf{- 0 . 6 0}[-\mathbf{6 . 5 3}, \mathbf{5 . 3 3}]\end{array}$

Heterogeneity: Not applicable

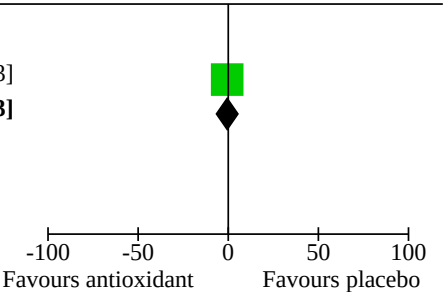


Analysis 1.5. Comparison 1: Antioxidants versus placebo in adults, Outcome 5: Tolerability of treatment - Dropouts due to adverse events

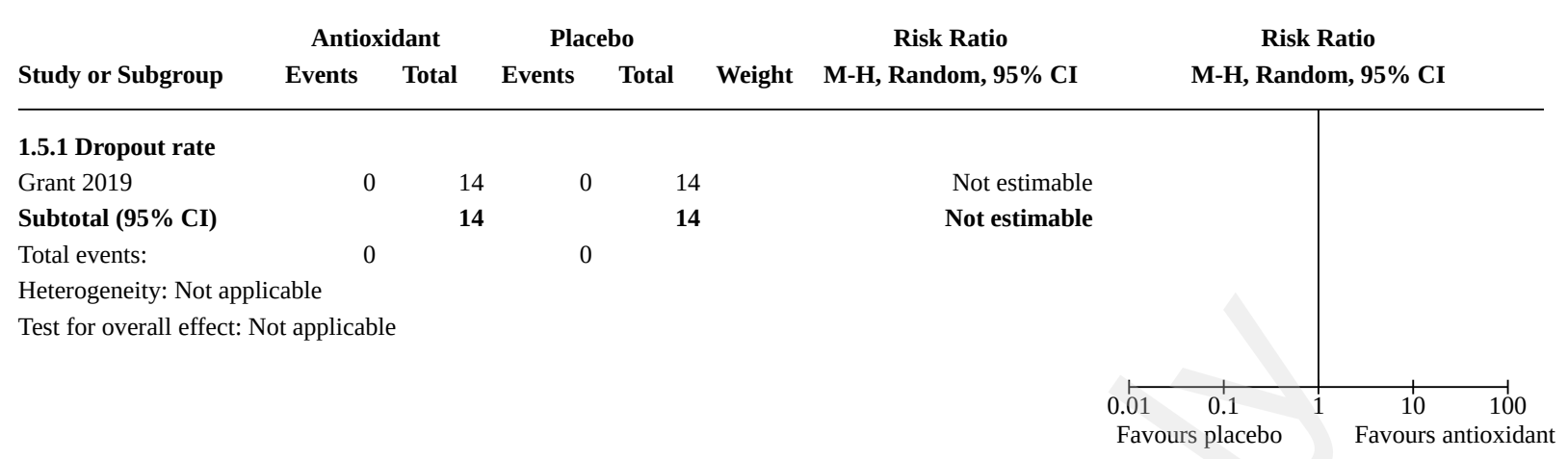

\section{Comparison 2. Antioxidants versus placebo in adolescents}

\begin{tabular}{|c|c|c|c|c|}
\hline Outcome or subgroup title & No. of studies & $\begin{array}{l}\text { No. of partici- } \\
\text { pants }\end{array}$ & Statistical method & Effect size \\
\hline 2.1 Treatment response & 1 & & $\begin{array}{l}\text { Risk Ratio (M-H, Random, } \\
95 \% \mathrm{Cl})\end{array}$ & Subtotals only \\
\hline $\begin{array}{l}\text { 2.1.1 Clinical Global Impressions - Im- } \\
\text { provement Scale (CGI-I) (no. of respon- } \\
\text { ders) }\end{array}$ & 1 & 8 & $\begin{array}{l}\text { Risk Ratio (M-H, Random, } \\
95 \% \mathrm{Cl})\end{array}$ & $2.00[0.28,14.20]$ \\
\hline 2.2 Reduction of TTM symptom severity & 1 & & $\begin{array}{l}\text { Mean Difference (IV, Ran- } \\
\text { dom, } 95 \% \mathrm{CI} \text { ) }\end{array}$ & Subtotals only \\
\hline $\begin{array}{l}\text { 2.2.1 Clinical Global Impression - Severi- } \\
\text { ty Scale (CGI-S) }\end{array}$ & 1 & 6 & $\begin{array}{l}\text { Mean Difference (IV, Ran- } \\
\text { dom, } 95 \% \mathrm{CI} \text { ) }\end{array}$ & $-1.13[-2.72,0.46]$ \\
\hline $\begin{array}{l}\text { 2.2.2 Massachusetts General Hospital } \\
\text { Hairpulling Scale (MGH-HPS) }\end{array}$ & 1 & 6 & $\begin{array}{l}\text { Mean Difference (IV, Ran- } \\
\text { dom, } 95 \% \mathrm{CI} \text { ) }\end{array}$ & $\begin{array}{l}-5.87[-17.46 \\
5.72]\end{array}$ \\
\hline $\begin{array}{l}\text { 2.2.3 NIMH Trichotillomania Severity } \\
\text { Scale (NIMH-TSS) }\end{array}$ & 1 & 6 & $\begin{array}{l}\text { Mean Difference (IV, Ran- } \\
\text { dom, } 95 \% \mathrm{CI} \text { ) }\end{array}$ & $-2.94[-8.14,2.26]$ \\
\hline $\begin{array}{l}\text { 2.2.4 Trichotillomania Scale For Chil- } \\
\text { dren - Child Version (TSC-C) }\end{array}$ & 1 & 6 & $\begin{array}{l}\text { Mean Difference (IV, Ran- } \\
\text { dom, } 95 \% \mathrm{CI} \text { ) }\end{array}$ & $0.00[-1.25,1.25]$ \\
\hline $\begin{array}{l}\text { 2.2.5 Trichotillomania Scale For Chil- } \\
\text { dren - Parent Version (TSC-P) }\end{array}$ & 1 & 4 & $\begin{array}{l}\text { Mean Difference (IV, Ran- } \\
\text { dom, } 95 \% \mathrm{CI} \text { ) }\end{array}$ & $\begin{array}{l}-1.20[-1.48 \\
-0.92]\end{array}$ \\
\hline $\begin{array}{l}2.3 \text { Reduction of comorbid symptoms of } \\
\text { depression }\end{array}$ & 1 & & $\begin{array}{l}\text { Mean Difference (IV, Ran- } \\
\text { dom, } 95 \% \mathrm{CI} \text { ) }\end{array}$ & Subtotals only \\
\hline $\begin{array}{l}\text { 2.3.1 Hamilton Depression Rating Scale } \\
\text { (HAM-D) }\end{array}$ & 1 & 7 & $\begin{array}{l}\text { Mean Difference (IV, Ran- } \\
\text { dom, } 95 \% \mathrm{CI} \text { ) }\end{array}$ & $-1.62[-3.98,0.74]$ \\
\hline 2.4 Quality of Life - functional disability & 1 & & $\begin{array}{l}\text { Mean Difference (IV, Ran- } \\
\text { dom, } 95 \% \mathrm{CI} \text { ) }\end{array}$ & Subtotals only \\
\hline 2.4.1 Sheehan Disability Scale (SDS) & 1 & 6 & $\begin{array}{l}\text { Mean Difference (IV, Ran- } \\
\text { dom, } 95 \% \mathrm{CI} \text { ) }\end{array}$ & $-1.30[-8.43,5.83]$ \\
\hline
\end{tabular}




\begin{tabular}{lllll}
\hline Outcome or subgroup title & No. of studies & $\begin{array}{l}\text { No. of partici- } \\
\text { pants }\end{array}$ & Statistical method & Effect size \\
\hline $\begin{array}{l}\text { 2.5 Tolerability of treatment - Dropouts } \\
\text { due to adverse events }\end{array}$ & 1 & & $\begin{array}{l}\text { Risk Ratio (M-H, Random, } \\
95 \% \mathrm{Cl})\end{array}$ & Subtotals only \\
\hline 2.5.1 Dropout rate & 1 & 8 & $\begin{array}{l}\text { Risk Ratio (M-H, Random, } \\
95 \% \mathrm{Cl})\end{array}$ & Not estimable \\
\hline
\end{tabular}

\section{Analysis 2.1. Comparison 2: Antioxidants versus placebo in adolescents, Outcome 1: Treatment response}

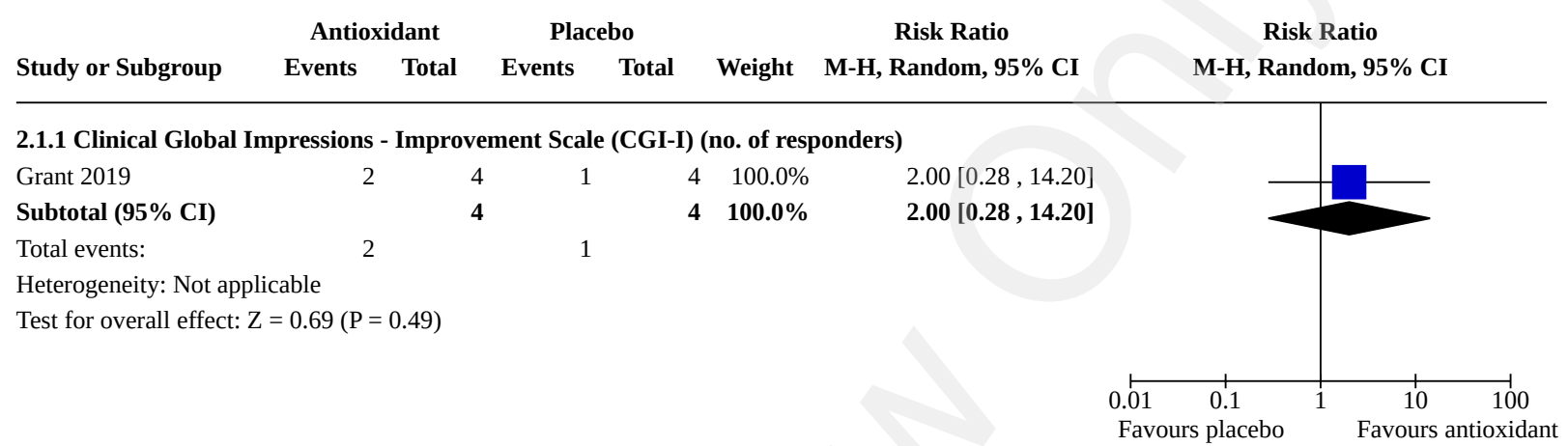




\section{Analysis 2.2. Comparison 2: Antioxidants versus placebo in} adolescents, Outcome 2: Reduction of TTM symptom severity

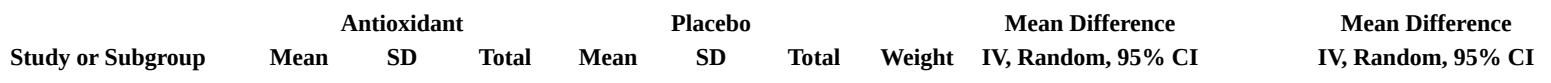

2.2.1 Clinical Global Impression - Severity Scale (CGI-S)

$\begin{array}{llllllll}\text { Grant } 2019 & 1.6 & 1.06 & 3 & 2.73 & 0.92 & 3 & 100.0 \%\end{array}$

Subtotal (95\% CI)

3

$\begin{array}{rrr}3 & 100.0 \% \\ 3 & \mathbf{1 0 0 . 0} \%\end{array}$

$-1.13[-2.72,0.46]$

Heterogeneity: Not applicable

Test for overall effect: $\mathrm{Z}=1.39(\mathrm{P}=0.16)$

2.2.2 Massachusetts General Hospital Hairpulling Scale (MGH-HPS)

Subtotal (95\% CI)

$\begin{array}{lll}6.83 & 8.5 \quad 3 \\ & 3\end{array}$

$12.7 \quad 5.71$

$3 \quad 100.0 \%$

$3 \quad 100.0 \%$

$-5.87[-17.46,5.72]$

$-5.87[-17.46,5.72]$

Heterogeneity: Not applicable

Test for overall effect: $Z=0.99(P=0.32)$

2.2.3 NIMH Trichotillomania Severity Scale (NIMH-TSS)

$\begin{array}{llllllll}\text { Grant } 2019 & 1.73 & 3.58 & 3 & 4.67 & 2.88 & 3 & 100.0 \%\end{array}$

Subtotal (95\% CI) 3

$\begin{array}{rr}3 & \mathbf{1 0 0 . 0} \%\end{array}$

$-2.94[-8.14,2.26]$

$-2.94[-8.14,2.26]$

Heterogeneity: Not applicable

Test for overall effect: $\mathrm{Z}=1.11(\mathrm{P}=0.27)$

2.2.4 Trichotillomania Scale For Children - Child Version (TSC-C)

$\begin{array}{lllllllll}\text { Grant } 2019 & 1.7 & 1.1 & 3 & 1.7 & 0.1 & 3 & 100.0 \% & 0.00[-1.25,1.25]\end{array}$

$\begin{array}{lll}3 & 100.0 \% & 0.00[-1.25,1.25\end{array}$

Heterogeneity: Not applicable

Test for overall effect: $\mathrm{Z}=0.00(\mathrm{P}=1.00)$

2.2.5 Trichotillomania Scale For Children - Parent Version (TSC-P)
Grant 2019
$1.1 \quad 1 \mathrm{e}-12$
2.3
0.2
$2 \quad 100.0 \%$
$-1.20[-1.48,-0.92]$

ubtotal (95\% CI)

$2 \quad 100.0 \%$

$-1.20[-1.48,-0.92]$

Heterogeneity: Not applicable

Test for overall effect: $\mathrm{Z}=8.49(\mathrm{P}<0.00001)$

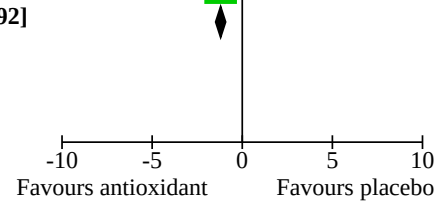

Analysis 2.3. Comparison 2: Antioxidants versus placebo in adolescents, Outcome 3: Reduction of comorbid symptoms of depression

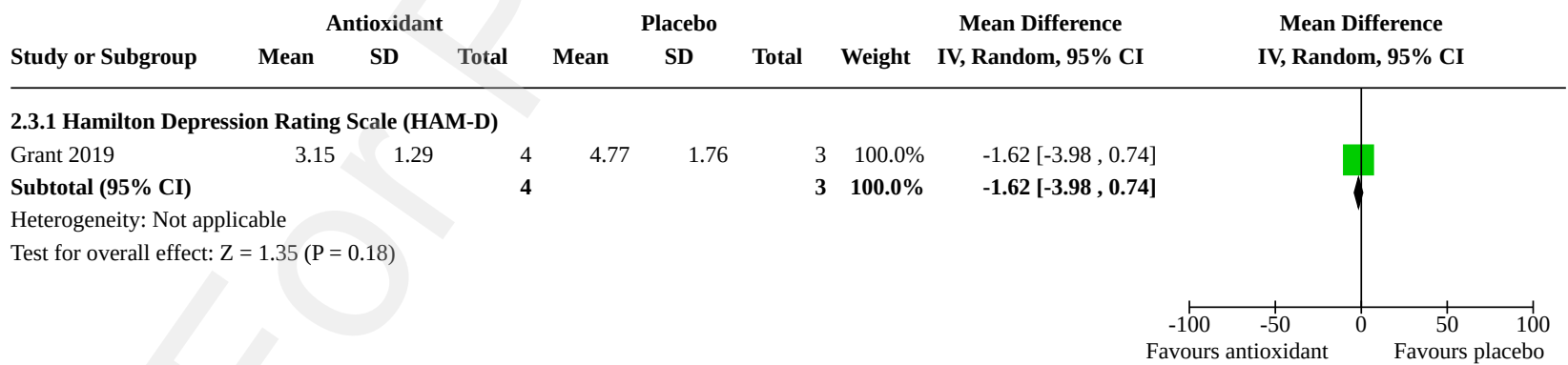




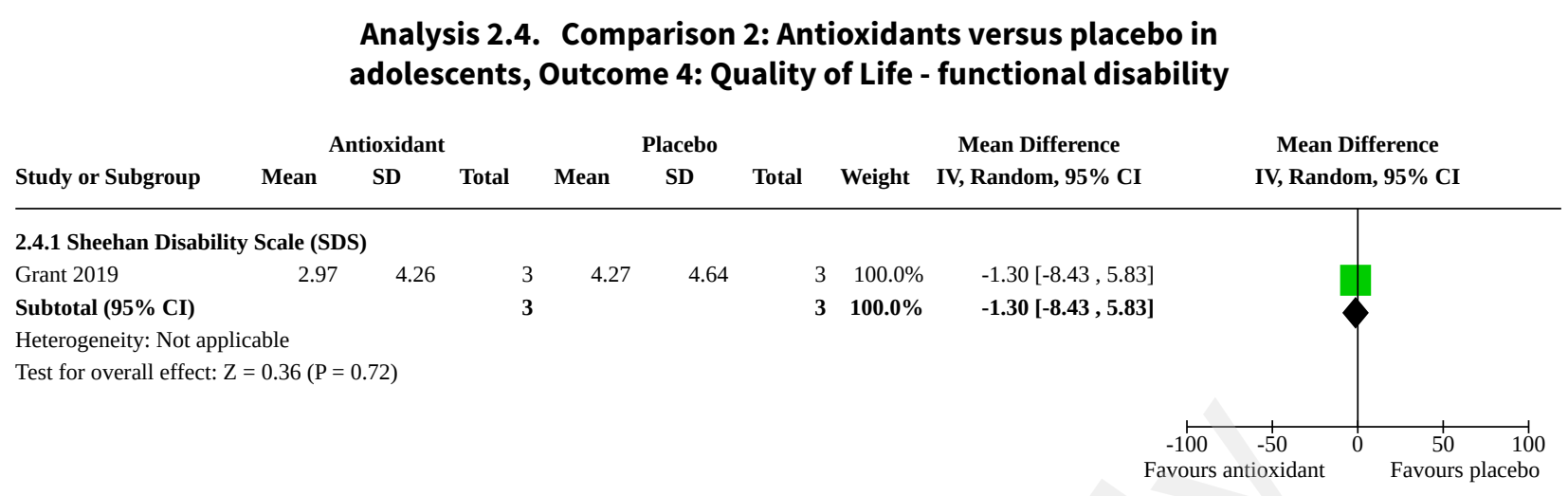
Analysis 2.5. Comparison 2: Antioxidants versus placebo in adolescents,
Outcome 5: Tolerability of treatment - Dropouts due to adverse events

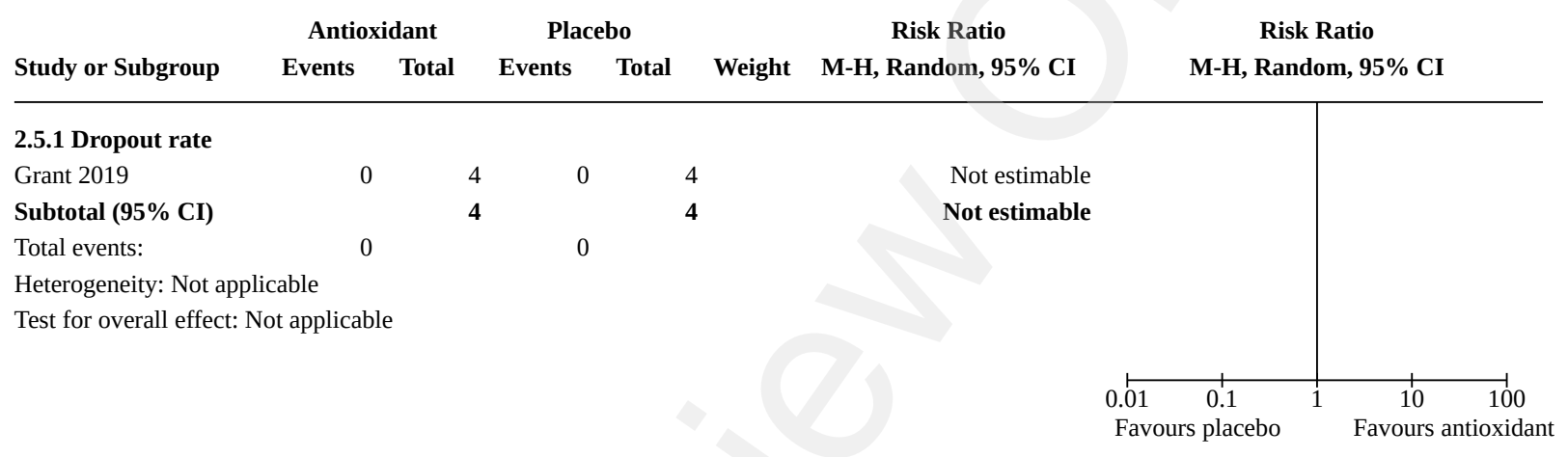

\section{Comparison 3. Antipsychotics versus placebo in adults}

\begin{tabular}{|c|c|c|c|c|}
\hline Outcome or subgroup title & No. of studies & $\begin{array}{l}\text { No. of partici- } \\
\text { pants }\end{array}$ & Statistical method & Effect size \\
\hline 3.1 Treatment response & 1 & & $\begin{array}{l}\text { Risk Ratio (M-H, Random, } \\
95 \% \mathrm{Cl})\end{array}$ & Subtotals only \\
\hline $\begin{array}{l}\text { 3.1.1 Clinical Global Impressions - Im- } \\
\text { provement Scale (CGI-I) (no. of respon- } \\
\text { ders) }\end{array}$ & 1 & 25 & $\begin{array}{l}\text { Risk Ratio (M-H, Random, } \\
95 \% \mathrm{Cl})\end{array}$ & $5.08[1.40,18.37]$ \\
\hline $\begin{array}{l}3.2 \text { Reduction of TTM symptom severi- } \\
\text { ty }\end{array}$ & 1 & & $\begin{array}{l}\text { Mean Difference (IV, Ran- } \\
\text { dom, 95\% CI) }\end{array}$ & Subtotals only \\
\hline $\begin{array}{l}\text { 3.2.1 Clinical Global Impressions - } \\
\text { Severity Scale (CGI-S) }\end{array}$ & 1 & 25 & $\begin{array}{l}\text { Mean Difference (IV, Ran- } \\
\text { dom, } 95 \% \mathrm{CI} \text { ) }\end{array}$ & $-1.68[-2.31,-1.05]$ \\
\hline $\begin{array}{l}\text { 3.2.2 Massachusetts General Hospital } \\
\text { Hairpulling Scale (MGH-HPS) }\end{array}$ & 1 & 25 & $\begin{array}{l}\text { Mean Difference (IV, Ran- } \\
\text { dom, } 95 \% \mathrm{CI} \text { ) }\end{array}$ & $-4.87[-8.84,-0.90]$ \\
\hline $\begin{array}{l}\text { 3.2.3 Yale-Brown Obsessive Compul- } \\
\text { sive Scale for Trichotillomania (TTM- } \\
\text { YBOCS) }\end{array}$ & 1 & 25 & $\begin{array}{l}\text { Mean Difference (IV, Ran- } \\
\text { dom, } 95 \% \mathrm{CI})\end{array}$ & $\begin{array}{l}-7.63[-12.35 \\
-2.91]\end{array}$ \\
\hline
\end{tabular}




\begin{tabular}{|c|c|c|c|c|}
\hline Outcome or subgroup title & No. of studies & $\begin{array}{l}\text { No. of partici- } \\
\text { pants }\end{array}$ & Statistical method & Effect size \\
\hline $\begin{array}{l}\text { 3.3 Tolerability of treatment - Dropouts } \\
\text { due to adverse events }\end{array}$ & 1 & & $\begin{array}{l}\text { Risk Ratio (M-H, Random, } \\
95 \% \mathrm{Cl})\end{array}$ & Subtotals only \\
\hline 3.3.1 Dropout rate & 1 & 25 & $\begin{array}{l}\text { Risk Ratio (M-H, Random, } \\
95 \% \mathrm{Cl})\end{array}$ & Not estimable \\
\hline $\begin{array}{l}\text { 3.4 Tolerability of treatment - Common } \\
\text { adverse events (> 20\%) }\end{array}$ & 1 & & $\begin{array}{l}\text { Risk Ratio (M-H, Random, } \\
95 \% \mathrm{Cl})\end{array}$ & Subtotals only \\
\hline 3.4.1 Dry mouth (no. of participants) & 1 & 25 & $\begin{array}{l}\text { Risk Ratio (M-H, Random, } \\
95 \% \mathrm{Cl})\end{array}$ & $\begin{array}{l}13.93[0.88 \\
220.37]\end{array}$ \\
\hline 3.4.2 Fatigue (no. of participants) & 1 & 25 & $\begin{array}{l}\text { Risk Ratio (M-H, Random, } \\
95 \% \mathrm{CI})\end{array}$ & $\begin{array}{l}13.93[0.88 \\
220.37]\end{array}$ \\
\hline $\begin{array}{l}\text { 3.4.3 Increased appetite (no. of partici- } \\
\text { pants) }\end{array}$ & 1 & 25 & $\begin{array}{l}\text { Risk Ratio (M-H, Random, } \\
95 \% \mathrm{Cl} \text { ) }\end{array}$ & $\begin{array}{l}12.07[0.75 \\
193.73]\end{array}$ \\
\hline 3.4.4 Headache (no. of participants) & 1 & 25 & $\begin{array}{l}\text { Risk Ratio (M-H, Random, } \\
95 \% \mathrm{Cl})\end{array}$ & $\begin{array}{l}10.21[0.62 \\
167.12]\end{array}$ \\
\hline 3.4.5 Weight gain (no. of participants) & 1 & 25 & $\begin{array}{l}\text { Risk Ratio (M-H, Random, } \\
95 \% \mathrm{Cl})\end{array}$ & $\begin{array}{l}10.21[0.62 \\
167.12]\end{array}$ \\
\hline
\end{tabular}

\section{Analysis 3.1. Comparison 3: Antipsychotics versus placebo in adults, Outcome 1: Treatment response}

\begin{tabular}{|c|c|c|c|c|c|c|c|}
\hline & Antips & hotics & & & & Risk Ratio & Risk Ratio \\
\hline Study or Subgroup & Events & Total & Events & Total & Weight & M-H, Random, 95\% CI & M-H, Random, 95\% CI \\
\hline
\end{tabular}

3.1.1 Clinical Global Impressions - Improvement Scale (CGI-I) (no. of responders)

$\begin{array}{lllllll}\text { Van Ameringen } 2010 & 11 & 13 & 2 & 12 & 100.0 \% & 5.08[1.40,18.37] \\ \text { Subtotal }(\mathbf{9 5 \%} \mathbf{C I}) & & \mathbf{1 3} & & \mathbf{1 2} & \mathbf{1 0 0 . 0 \%} & \mathbf{5 . 0 8}[\mathbf{1 . 4 0}, \mathbf{1 8 . 3 7}] \\ \text { Total events: } & 11 & & 2 & & & \\ \text { Heterogeneity: Not applicable } & & & & & & \\ \text { Test for overall effect: } \mathrm{Z}=2.48(\mathrm{P}=0.01) & & & & & \end{array}$


Analysis 3.2. Comparison 3: Antipsychotics versus placebo in adults, Outcome 2: Reduction of TTM symptom severity

\begin{tabular}{|c|c|c|c|c|c|c|c|c|c|}
\hline & \multicolumn{3}{|c|}{ Antipsychotics } & \multicolumn{3}{|c|}{ Placebo } & \multicolumn{2}{|r|}{ Mean Difference } & Mean Difference \\
\hline Study or Subgroup & Mean & SD & Total & Mean & SD & Total & Weight & IV, Random, 95\% CI & IV, Random, 95\% CI \\
\hline
\end{tabular}

3.2.1 Clinical Global Impressions - Severity Scale (CGI-S)

$\begin{array}{lllllllll}\text { Van Ameringen } 2010 & 3.15 & 0.9 & 13 & 4.83 & 0.71 & 12 & 100.0 \% & -1.68[-2.31,-1.05]\end{array}$

$\begin{array}{lllll}\text { Subtotal }(95 \% \mathrm{CI}) & 13 & 12 & 100.0 \% & -1.68[-2.31,-1.05]\end{array}$

Heterogeneity: Not applicable

Test for overall effect: $\mathrm{Z}=5.20(\mathrm{P}<0.00001)$

3.2.2 Massachusetts General Hospital Hairpulling Scale (MGH-HPS)

$\begin{array}{lllllllll}\text { Van Ameringen } 2010 & 8.38 & 4.23 & 13 & 13.25 & 5.72 & 12 & 100.0 \% & -4.87[-8.84,-0.90]\end{array}$

Subtotal (95\% CI)

$13 \quad 12 \quad 100.0 \%$

$-4.87[-8.84,-0.90]$

Heterogeneity: Not applicable

Test for overall effect: $\mathrm{Z}=2.40(\mathrm{P}=0.02)$

3.2.3 Yale-Brown Obsessive Compulsive Scale for Trichotillomania (TTM-YBOCS)
Van Ameringen 2010
10.54
6.74
13
$18.17 \quad 5.27$
$12 \quad 100.0 \%$
$-7.63[-12.35,-2.91]$

Subtotal (95\% CI)

13

$12 \quad 100.0 \%$

$-7.63[-12.35,-2.91]$

Heterogeneity: Not applicable

Test for overall effect: $\mathrm{Z}=3.17(\mathrm{P}=0.002)$

Analysis 3.3. Comparison 3: Antipsychotics versus placebo in adults, Outcome 3: Tolerability of treatment - Dropouts due to adverse events

\begin{tabular}{|c|c|c|c|c|c|c|c|}
\hline & Antip & hotics & $\mathbf{P l a}$ & & & Risk Ratio & Risk Ratio \\
\hline Study or Subgroup & Events & Total & Events & Total & Weight & M-H, Random, 95\% CI & M-H, Random, 95\% CI \\
\hline
\end{tabular}

\subsubsection{Dropout rate}

Van Ameringen 2010

Subtotal (95\% CI)

0

Not estimable

Total events:

Heterogeneity: Not applicable

Test for overall effect: Not applicable

0 
Analysis 3.4. Comparison 3: Antipsychotics versus placebo in adults, Outcome 4: Tolerability of treatment - Common adverse events ( $>20 \%)$

\begin{tabular}{lccccccc} 
& \multicolumn{2}{c}{ Antipsychotics } & \multicolumn{2}{c}{ Placebo } & \multicolumn{2}{c}{ Risk Ratio } & Risk Ratio \\
Study or Subgroup & Events & Total & Events & Total & Weight & M-H, Random, 95\% CI & M-H, Random, 95\% CI
\end{tabular}

3.4.1 Dry mouth (no. of participants)

Subtotal (95\% CI)

$\begin{array}{llll}13 & 0 & 12 & 100.0 \%\end{array}$

$13.93[0.88,220.37]$

Total events:

Heterogeneity: Not applicable

Test for overall effect: $\mathrm{Z}=1.87(\mathrm{P}=0.06)$

3.4.2 Fatigue (no. of participants)

Van Ameringen 2010

Subtotal (95\% CI)

0

Total events:

13

13

Heterogeneity: Not applicable

Test for overall effect: $\mathrm{Z}=1.87(\mathrm{P}=0.06)$

3.4.3 Increased appetite (no. of participants)

$\begin{array}{lll}\text { Van Ameringen } 2010 & 6 & 13 \\ \text { Subtotal (95\% CI) } & & \mathbf{1 3}\end{array}$

Subtotal (95\% CI)

Total events:

6

Heterogeneity: Not applicable

Test for overall effect: $\mathrm{Z}=1.76(\mathrm{P}=0.08)$

3.4.4 Headache (no. of participants)

Van Ameringen $2010 \quad 5$

Subtotal (95\% CI)

$5 \quad 13$

0

$12 \quad 100.0 \%$

$10.21[0.62,167.12]$

Total events: 5

Heterogeneity: Not applicable

Test for overall effect: $\mathrm{Z}=1.63(\mathrm{P}=0.10)$

3.4.5 Weight gain (no. of participants)

Van Ameringen $2010 \quad 5$

Subtotal (95\% CI)

$5 \quad 13$

0

$12 \quad 100.0 \%$

$12.07[0.75,193.73]$

$13.93[0.88,220.37]$

$13.93[0.88,220.37]$

Total events:

5

Heterogeneity: Not applicable

Test for overall effect: $\mathrm{Z}=1.63(\mathrm{P}=0.10)$

$12 \quad 100.0 \% \quad 12.07[0.75,193.73]$

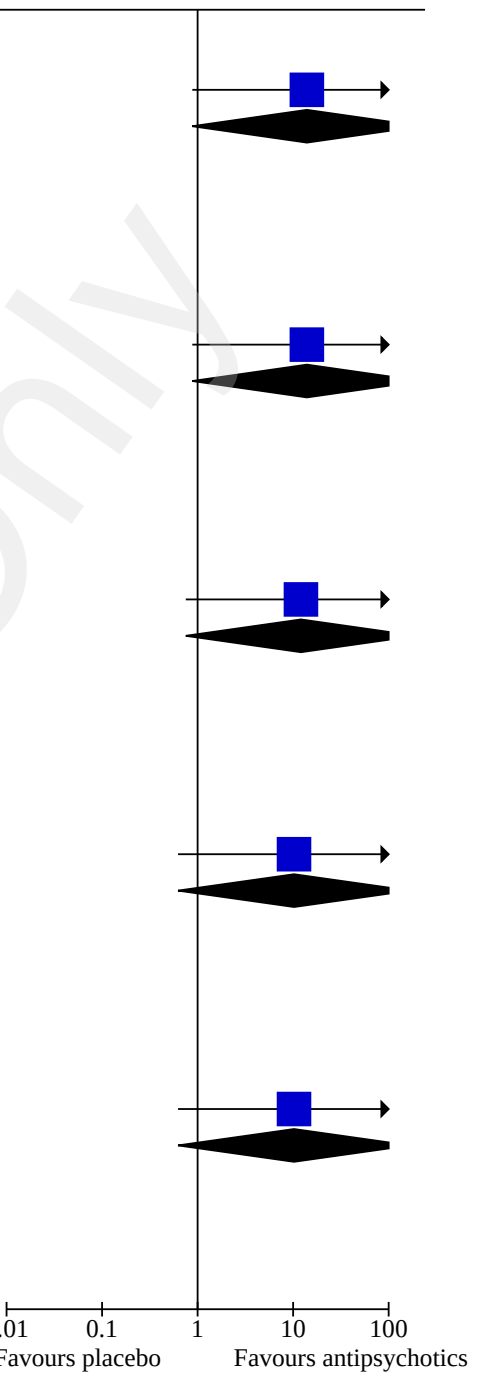

Comparison 4. Cell signal transducers versus placebo in adults

\begin{tabular}{lllll}
\hline Outcome or subgroup title & No. of studies & $\begin{array}{l}\text { No. of partici- } \\
\text { pants }\end{array}$ & Statistical method & Effect size \\
\hline 4.1 Treatment response & 1 & & $\begin{array}{l}\text { Risk Ratio (M-H, Random, } \\
95 \% \mathrm{Cl})\end{array}$ & Subtotals only \\
\hline $\begin{array}{l}\text { 4.1.1 Clinical Global Impressions - Im- } \\
\text { provement Scale (CGI-I) (no. of respon- } \\
\text { ders) }\end{array}$ & 1 & 38 & $\begin{array}{l}\text { Risk Ratio (M-H, Random, } \\
95 \% \mathrm{Cl})\end{array}$ & $1.33[0.57,3.11]$ \\
\hline $\begin{array}{l}\text { 4.2 Reduction of TTM symptom severity } \\
\text { n }\end{array}$ & 1 & & $\begin{array}{l}\text { Mean Difference (IV, Ran- } \\
\text { dom, 95\% Cl) }\end{array}$ & Subtotals only \\
\hline
\end{tabular}




\begin{tabular}{|c|c|c|c|c|}
\hline Outcome or subgroup title & No. of studies & $\begin{array}{l}\text { No. of partici- } \\
\text { pants }\end{array}$ & Statistical method & Effect size \\
\hline $\begin{array}{l}\text { 4.2.1 Massachusetts General Hospital } \\
\text { Hairpulling Scale (MGH-HPS) }\end{array}$ & 1 & 38 & $\begin{array}{l}\text { Mean Difference (IV, Ran- } \\
\text { dom, } 95 \% \mathrm{CI} \text { ) }\end{array}$ & $-1.30[-5.12,2.52]$ \\
\hline $\begin{array}{l}\text { 4.2.2 NIMH Trichotillomania Severity } \\
\text { Scale (NIMH-TSS) }\end{array}$ & 1 & 38 & $\begin{array}{l}\text { Mean Difference (IV, Ran- } \\
\text { dom, } 95 \% \mathrm{CI} \text { ) }\end{array}$ & $0.00[-3.37,3.37]$ \\
\hline $\begin{array}{l}\text { 4.3 Tolerability of treatment - Dropouts } \\
\text { due to adverse events }\end{array}$ & 1 & & $\begin{array}{l}\text { Risk Ratio (M-H, Random, } \\
95 \% \mathrm{Cl})\end{array}$ & Subtotals only \\
\hline 4.3.1 Dropout rate & 1 & 38 & $\begin{array}{l}\text { Risk Ratio (M-H, Random, } \\
95 \% \mathrm{Cl} \text { ) }\end{array}$ & Not estimable \\
\hline $\begin{array}{l}\text { 4.4 Tolerability of treatment - Common } \\
\text { adverse events }(>20 \%)\end{array}$ & 1 & & $\begin{array}{l}\text { Risk Ratio (M-H, Random, } \\
95 \% \mathrm{CI})\end{array}$ & Subtotals only \\
\hline $\begin{array}{l}\text { 4.4.1 Nausea / upset stomach (no. of } \\
\text { participants) }\end{array}$ & 1 & 38 & $\begin{array}{l}\text { Risk Ratio (M-H, Random, } \\
95 \% \mathrm{Cl} \text { ) }\end{array}$ & $2.00[0.41,9.65]$ \\
\hline
\end{tabular}

Analysis 4.1. Comparison 4: Cell signal transducers versus placebo in adults, Outcome 1: Treatment response

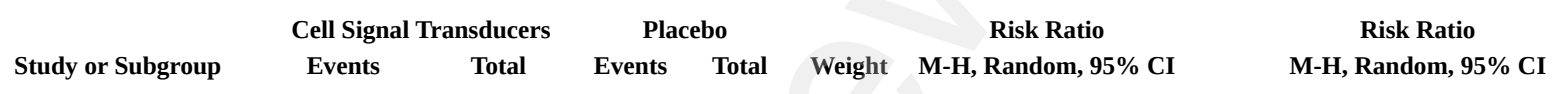

4.1.1 Clinical Global Impressions - Improvement Scale (CGI-I) (no. of responders)

$\begin{array}{llllll}\text { Leppink } 2017 & 8 & 19 & 6 & 19 & 100.0 \%\end{array}$

$\begin{array}{llll}19 & 19-100.0 \% & 1.33[0.57,3.11]\end{array}$

Total events: $\quad 8$

Heterogeneity: Not applicable

Test for overall effect: $\mathrm{Z}=0.67(\mathrm{P}=0.51)$

6

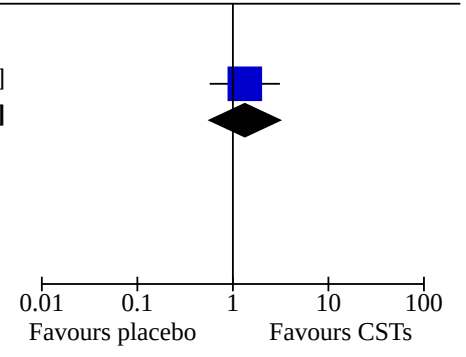

Analysis 4.2. Comparison 4: Cell signal transducers versus placebo in adults, Outcome 2: Reduction of TTM symptom severity

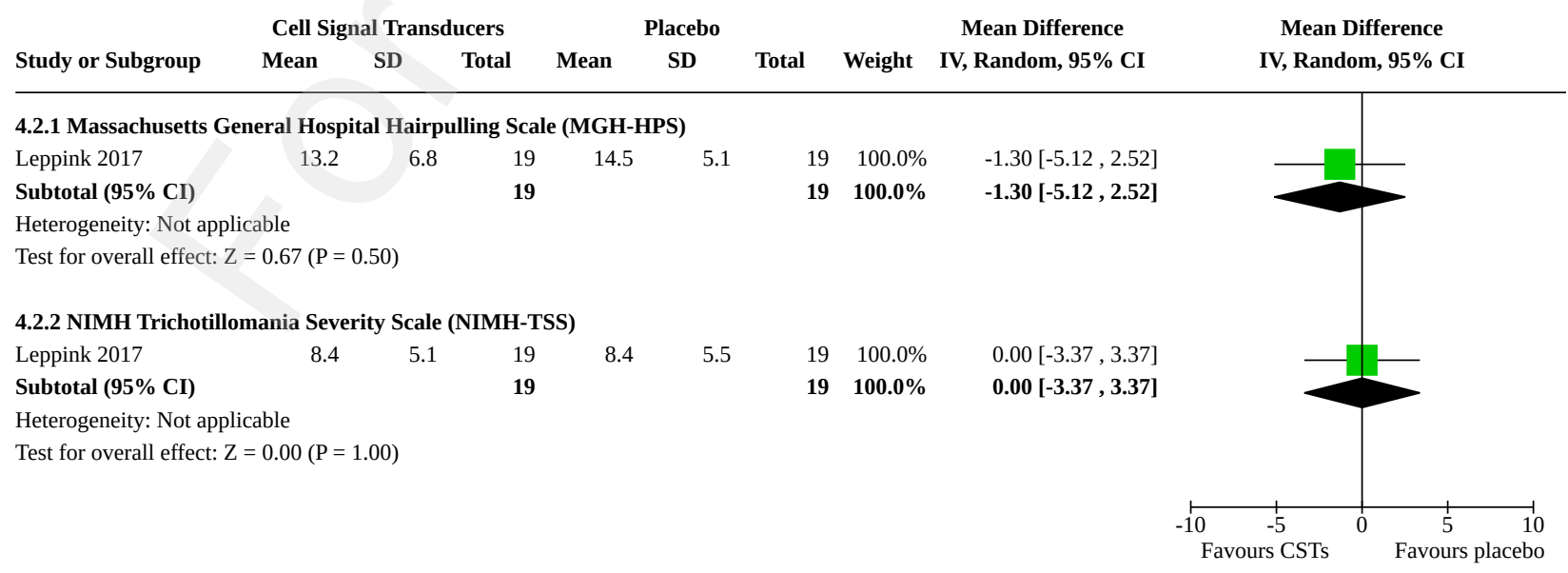


Analysis 4.3. Comparison 4: Cell signal transducers versus placebo in adults, Outcome 3: Tolerability of treatment - Dropouts due to adverse events

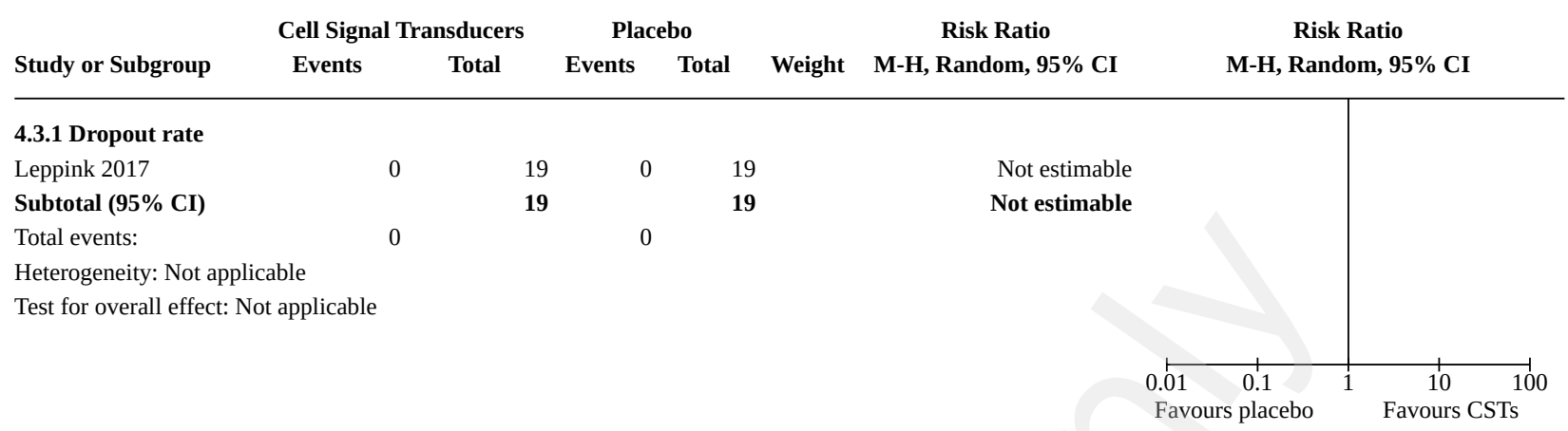

\section{Analysis 4.4. Comparison 4: Cell signal transducers versus placebo in adults, Outcome 4: Tolerability of treatment - Common adverse events (> 20\%)}

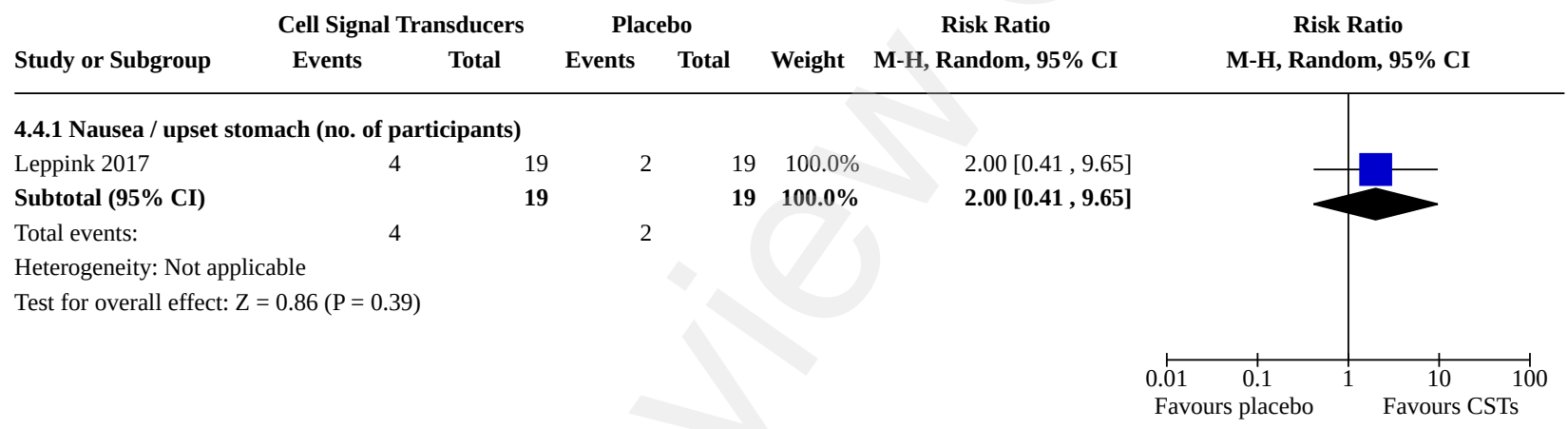

Comparison 5. Glutamate modulators versus placebo in adults

\begin{tabular}{|c|c|c|c|c|}
\hline Outcome or subgroup title & No. of studies & $\begin{array}{l}\text { No. of partici- } \\
\text { pants }\end{array}$ & Statistical method & Effect size \\
\hline 5.1 Treatment response & 1 & & $\begin{array}{l}\text { Risk Ratio (M-H, Random, } \\
95 \% \mathrm{Cl})\end{array}$ & Subtotals only \\
\hline $\begin{array}{l}\text { 5.1.1 Clinical Global Impressions - Im- } \\
\text { provement Scale (CGI-I) (no. of respon- } \\
\text { ders) }\end{array}$ & 1 & 50 & $\begin{array}{l}\text { Risk Ratio (M-H, Random, } \\
95 \% \mathrm{Cl})\end{array}$ & $3.50[1.34,9.17]$ \\
\hline $\begin{array}{l}5.2 \text { Reduction of TTM symptom severi- } \\
\text { ty }\end{array}$ & 1 & & $\begin{array}{l}\text { Mean Difference (IV, Random, } \\
95 \% \mathrm{CI})\end{array}$ & Subtotals only \\
\hline $\begin{array}{l}\text { 5.2.1 Clinical Global Impression - } \\
\text { Severity Scale (CGI-S) }\end{array}$ & 1 & 50 & $\begin{array}{l}\text { Mean Difference (IV, Random, } \\
95 \% \mathrm{CI})\end{array}$ & $\begin{array}{l}-1.24[-1.93 \\
-0.55]\end{array}$ \\
\hline $\begin{array}{l}\text { 5.2.2 Massachusetts General Hospital } \\
\text { Hairpulling Scale (MGH-HPS) }\end{array}$ & 1 & 50 & $\begin{array}{l}\text { Mean Difference (IV, Random, } \\
95 \% \mathrm{CI})\end{array}$ & $\begin{array}{l}-5.60[-8.50 \\
-2.70]\end{array}$ \\
\hline $\begin{array}{l}\text { 5.2.3 Psychiatric Institute Trichotillo- } \\
\text { mania Scale (PITS) }\end{array}$ & 1 & 50 & $\begin{array}{l}\text { Mean Difference (IV, Random, } \\
95 \% \mathrm{CI} \text { ) }\end{array}$ & $\begin{array}{l}-6.90[-10.56 \\
-3.24]\end{array}$ \\
\hline
\end{tabular}

Pharmacotherapy for trichotillomania (Review) 


\begin{tabular}{|c|c|c|c|c|}
\hline Outcome or subgroup title & No. of studies & $\begin{array}{l}\text { No. of partici- } \\
\text { pants }\end{array}$ & Statistical method & Effect size \\
\hline $\begin{array}{l}5.3 \text { Reduction of comorbid symptoms } \\
\text { of depression }\end{array}$ & 1 & & $\begin{array}{l}\text { Mean Difference (IV, Random, } \\
95 \% \mathrm{CI} \text { ) }\end{array}$ & Subtotals only \\
\hline $\begin{array}{l}\text { 5.3.1 Hamilton Depression Rating } \\
\text { Scale (HAM-D) }\end{array}$ & 1 & 50 & $\begin{array}{l}\text { Mean Difference (IV, Random, } \\
95 \% \mathrm{CI} \text { ) }\end{array}$ & $-0.96[-2.88,0.96]$ \\
\hline 5.4 Quality of Life - functional disability & 1 & & $\begin{array}{l}\text { Mean Difference (IV, Random, } \\
95 \% \mathrm{Cl} \text { ) }\end{array}$ & Subtotals only \\
\hline 5.4.1 Sheehan Disability Scale (SDS) & 1 & 50 & $\begin{array}{l}\text { Mean Difference (IV, Random, } \\
95 \% \mathrm{CI} \text { ) }\end{array}$ & $-2.76[-5.58,0.06]$ \\
\hline $\begin{array}{l}\text { 5.5 Tolerability of treatment - Dropouts } \\
\text { due to adverse events }\end{array}$ & 1 & & $\begin{array}{l}\text { Risk Ratio (M-H, Random, } \\
95 \% \mathrm{Cl})\end{array}$ & Subtotals only \\
\hline 5.5.1 Dropout rate & 1 & 50 & $\begin{array}{l}\text { Risk Ratio (M-H, Random, } \\
95 \% \mathrm{Cl} \text { ) }\end{array}$ & Not estimable \\
\hline
\end{tabular}

\section{Analysis 5.1. Comparison 5: Glutamate modulators versus placebo in adults, Outcome 1: Treatment response}

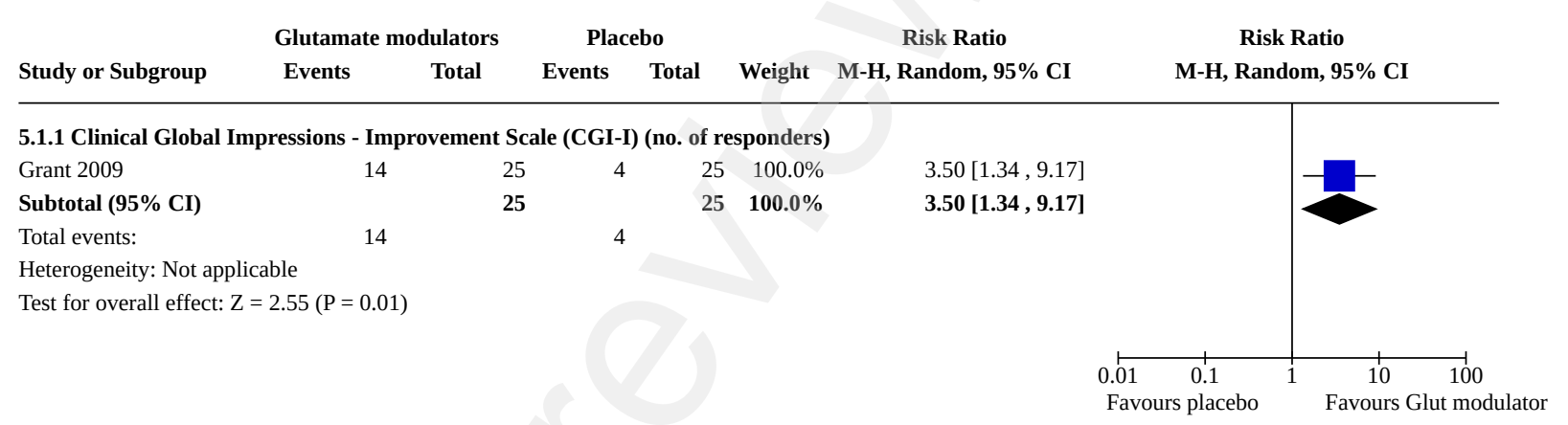


Analysis 5.2. Comparison 5: Glutamate modulators versus placebo in adults, Outcome 2: Reduction of TTM symptom severity

\begin{tabular}{|c|c|c|c|c|c|c|c|}
\hline & Glut & te $\mathbf{m}$ & ators & & & Mean Difference & Mean Difference \\
\hline Study or Subgroup & Mean & SD & Total & Mean & Weight & IV, Random, 95\% CI & IV, Random, 95\% CI \\
\hline
\end{tabular}

5.2.1 Clinical Global Impression - Severity Scale (CGI-S)

$\begin{array}{lllllllll}\text { Grant } 2009 & 2.96 & 1.379 & 25 & 4.2 & 1.08 & 25 & 100.0 \% & -1.24[-1.93,-0.55] \\ \text { Subtotal }(\mathbf{9 5} \% \mathbf{C I}) & & & \mathbf{2 5} & & & \mathbf{2 5} & \mathbf{1 0 0 . 0 \%} & \mathbf{- 1 . 2 4}[\mathbf{- 1 . 9 3}, \mathbf{- 0 . 5 5}]\end{array}$

Subtotal (95\% C

Heterogeneity: Not applicable

Test for overall effect: $\mathrm{Z}=3.54(\mathrm{P}=0.0004)$

5.2.2 Massachusetts General Hospital Hairpulling Scale (MGH-HPS)

$\begin{array}{lllllllll}\text { Grant } 2009 & 10.4 & 5.55 & 25 & 16 & 4.9 & 25 & 100.0 \% & -5.60[-8.50,-2.70] \\ \text { Subtotal (95\% CI) } & & & \mathbf{2 5} & & & \mathbf{2 5} & \mathbf{1 0 0 . 0} \% & \mathbf{- 5 . 6 0}[\mathbf{- 8 . 5 0}, \mathbf{- 2 . 7 0}]\end{array}$

25

$25 \quad 100.0 \%$

$-5.60[-8.50,-2.70]$

Heterogeneity: Not applicable

Test for overall effect: $\mathrm{Z}=3.78(\mathrm{P}=0.0002)$

5.2.3 Psychiatric Institute Trichotillomania Scale (PITS)

$\begin{array}{lllllllll}\text { Grant } 2009 & 11.8 & 7.61 & 25 & 18.7 & 5.41 & 25 & 100.0 \% & -6.90[-10.56,-3.24]\end{array}$

Subtotal (95\% CI)

25

$25 \quad 100.0 \%$

$-6.90[-10.56,-3.24]$

Heterogeneity: Not applicable

Test for overall effect: $Z=3.69(P=0.0002)$

Analysis 5.3. Comparison 5: Glutamate modulators versus placebo in adults, Outcome 3: Reduction of comorbid symptoms of depression

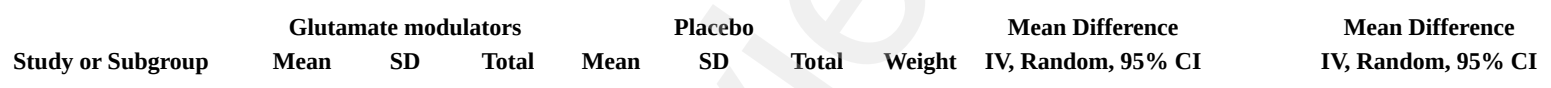

5.3.1 Hamilton Depression Rating Scale (HAM-D)

$\begin{array}{llllllllll}\text { Grant } 2009 & 2.36 & 2.98 & 25 & 3.32 & 3.9 & 25 & 100.0 \% & -0.96[-2.88,0.96]\end{array}$

Subtotal (95\% CI)

$25 \quad 100.0 \%$

$-0.96[-2.88,0.96]$

Heterogeneity: Not applicable

Test for overall effect: $\mathrm{Z}=0.98(\mathrm{P}=0.33)$

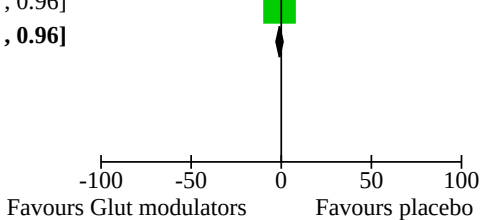

Analysis 5.4. Comparison 5: Glutamate modulators versus placebo in adults, Outcome 4: Quality of Life - functional disability

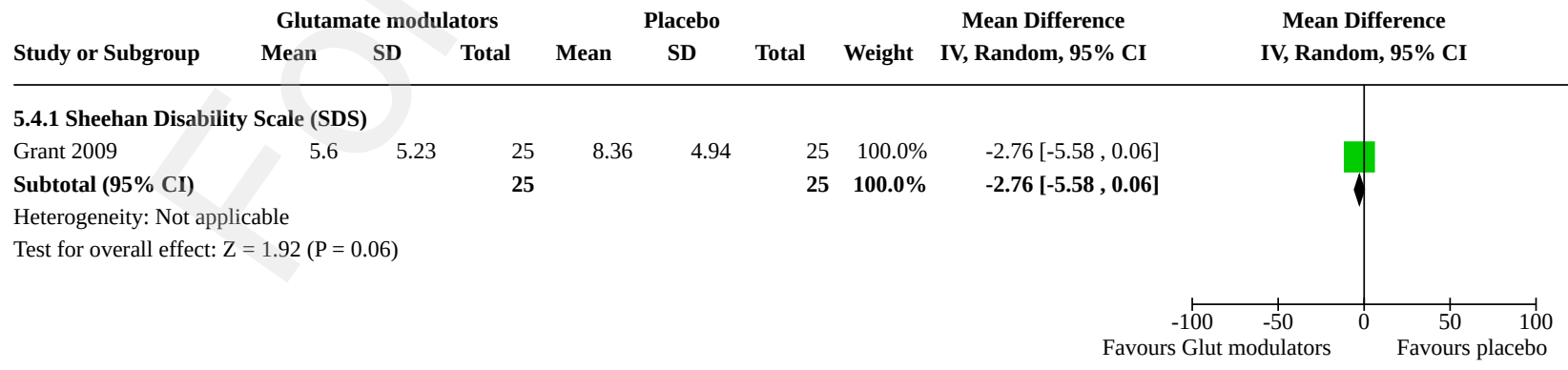


Analysis 5.5. Comparison 5: Glutamate modulators versus placebo in adults, Outcome 5: Tolerability of treatment - Dropouts due to adverse events

\begin{tabular}{cccccccc} 
& \multicolumn{2}{c}{ Glutamate modulators } & \multicolumn{2}{c}{ Placebo } & \multicolumn{2}{c}{ Risk Ratio } & Risk Ratio \\
Study or Subgroup & Events & Total & Events & Total & Weight & M-H, Random, 95\% CI & M-H, Random, 95\% CI
\end{tabular}

5.5.1 Dropout rate

Grant 2009

Subtotal (95\% CI)

Total events:

$\begin{array}{llllr}0 & 25 & 0 & 25 & \text { Not estimable } \\ & 25 & & 25 & \text { Not estimable } \\ 0 & & 0 & & \end{array}$

0

Not estimable

Heterogeneity: Not applicable

Test for overall effect: Not applicable

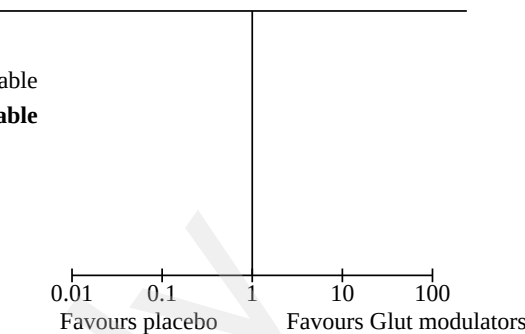

Comparison 6. Glutamate modulators versus placebo in children and adolescents

\begin{tabular}{|c|c|c|c|c|}
\hline Outcome or subgroup title & No. of studies & $\begin{array}{l}\text { No. of partici- } \\
\text { pants }\end{array}$ & Statistical method & Effect size \\
\hline 6.1 Treatment response & 1 & & $\begin{array}{l}\text { Risk Ratio (M-H, Random, } \\
95 \% \mathrm{Cl} \text { ) }\end{array}$ & Subtotals only \\
\hline $\begin{array}{l}\text { 6.1.1 Clinical Global Impressions - Im- } \\
\text { provement Scale (CGI-I) (no. of respon- } \\
\text { ders) }\end{array}$ & 1 & 39 & $\begin{array}{l}\text { Risk Ratio (M-H, Random, } \\
95 \% \mathrm{Cl})\end{array}$ & $1.19[0.37,3.77]$ \\
\hline 6.2 Reduction of TTM symptom severity & 1 & & $\begin{array}{l}\text { Mean Difference (IV, Ran- } \\
\text { dom, } 95 \% \mathrm{CI} \text { ) }\end{array}$ & Subtotals only \\
\hline $\begin{array}{l}\text { 6.2.1 Massachusetts General Hospital } \\
\text { Hairpulling Scale (MGH-HPS) }\end{array}$ & 1 & 39 & $\begin{array}{l}\text { Mean Difference (IV, Ran- } \\
\text { dom, } 95 \% \mathrm{CI} \text { ) }\end{array}$ & $\begin{array}{l}-2.83[-3.76 \\
-1.90]\end{array}$ \\
\hline $\begin{array}{l}\text { 6.2.2 NIMH Trichotillomania Severity } \\
\text { Scale (NIMH-TSS) }\end{array}$ & 1 & 39 & $\begin{array}{l}\text { Mean Difference (IV, Ran- } \\
\text { dom, } 95 \% \mathrm{CI} \text { ) }\end{array}$ & $\begin{array}{l}-1.33[-1.97 \\
-0.69]\end{array}$ \\
\hline $\begin{array}{l}\text { 6.2.3 Trichotillomania Scale for Children } \\
\text { - Child version (TSC-C) }\end{array}$ & 1 & 39 & $\begin{array}{l}\text { Mean Difference (IV, Ran- } \\
\text { dom, } 95 \% \mathrm{CI} \text { ) }\end{array}$ & $-0.08[-0.22,0.06]$ \\
\hline $\begin{array}{l}\text { 6.2.4 Trichotillomania Scale for Children } \\
\text { - Parent version (TSC-P) }\end{array}$ & 1 & 39 & $\begin{array}{l}\text { Mean Difference (IV, Ran- } \\
\text { dom, } 95 \% \mathrm{CI} \text { ) }\end{array}$ & $-0.05[-0.16,0.06]$ \\
\hline $\begin{array}{l}\text { 6.3 Reduction of comorbid symptoms of } \\
\text { depression }\end{array}$ & 1 & & $\begin{array}{l}\text { Mean Difference (IV, Ran- } \\
\text { dom, } 95 \% \mathrm{CI} \text { ) }\end{array}$ & Subtotals only \\
\hline $\begin{array}{l}\text { 6.3.1 Children's Depression Inventory } \\
\text { (CDI) }\end{array}$ & 1 & 39 & $\begin{array}{l}\text { Mean Difference (IV, Ran- } \\
\text { dom, } 95 \% \mathrm{CI} \text { ) }\end{array}$ & $3.10[1.91,4.29]$ \\
\hline $\begin{array}{l}\text { 6.4 Tolerability of treatment - Dropouts } \\
\text { due to adverse eventsDropouts due to } \\
\text { adverse events }\end{array}$ & 1 & & $\begin{array}{l}\text { Risk Ratio (M-H, Random, } \\
95 \% \mathrm{Cl})\end{array}$ & Subtotals only \\
\hline 6.4.1 Dropout rate & 1 & 39 & $\begin{array}{l}\text { Risk Ratio (M-H, Random, } \\
95 \% \mathrm{Cl} \text { ) }\end{array}$ & $2.86[0.12,66.11]$ \\
\hline $\begin{array}{l}6.5 \text { Tolerability of treatment - Common } \\
\text { adverse events }(>20 \%)\end{array}$ & 1 & & $\begin{array}{l}\text { Risk Ratio (M-H, Random, } \\
95 \% \mathrm{Cl} \text { ) }\end{array}$ & Subtotals only \\
\hline
\end{tabular}




\begin{tabular}{lllll}
\hline Outcome or subgroup title & No. of studies & $\begin{array}{l}\text { No. of partici- } \\
\text { pants }\end{array}$ & Statistical method & Effect size \\
\hline 6.5.1 Nausea (no. of participants) & 1 & 39 & $\begin{array}{l}\text { Risk Ratio (M-H, Random, } \\
95 \% \mathrm{Cl})\end{array}$ & $0.48[0.22,1.01]$ \\
\hline
\end{tabular}

\section{Analysis 6.1. Comparison 6: Glutamate modulators versus placebo in children and adolescents, Outcome 1: Treatment response}

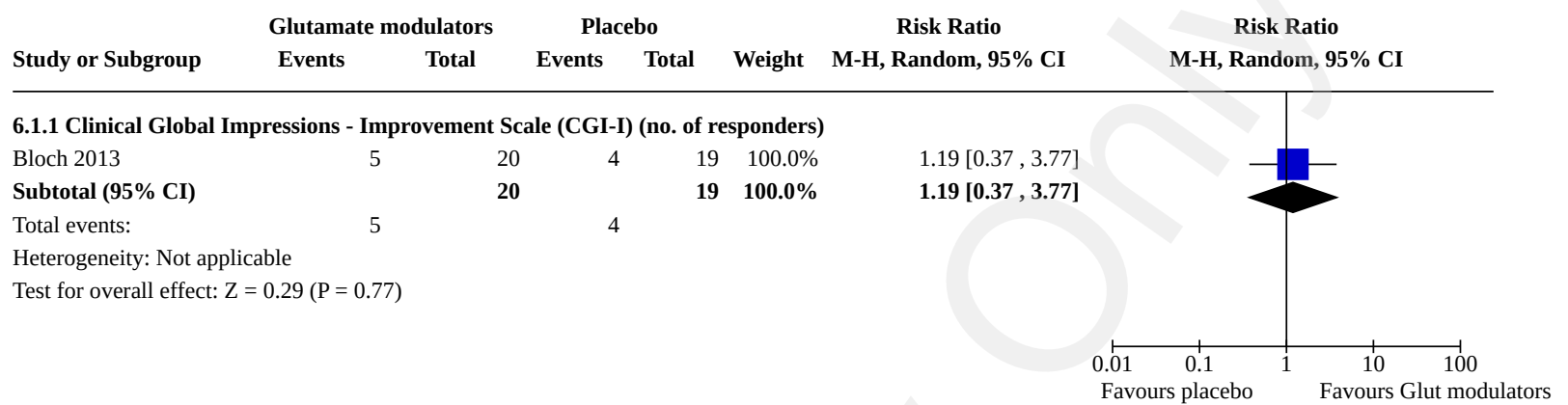

Analysis 6.2. Comparison 6: Glutamate modulators versus placebo in children and adolescents, Outcome 2: Reduction of TTM symptom severity

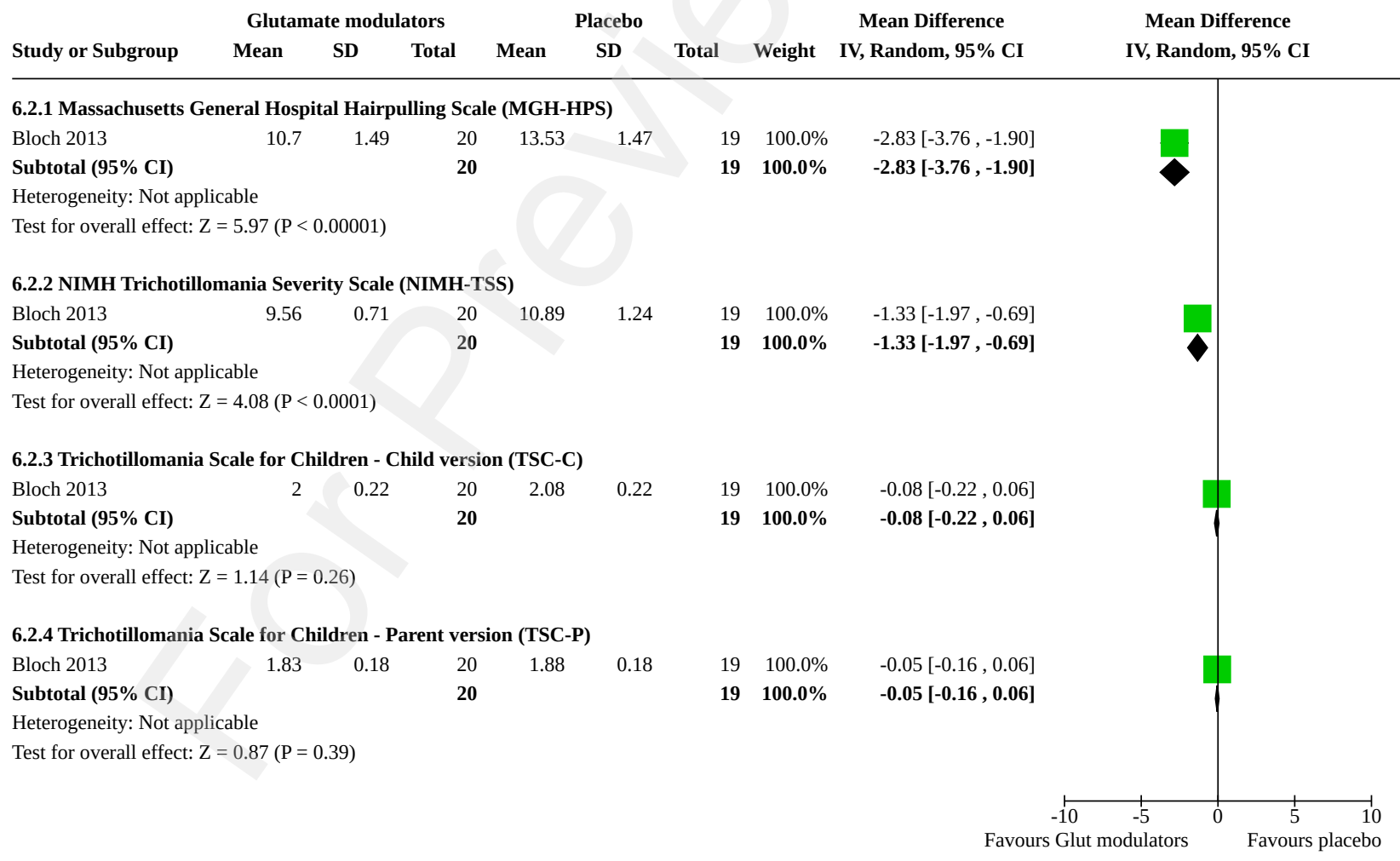


Analysis 6.3. Comparison 6: Glutamate modulators versus placebo in children and adolescents, Outcome 3: Reduction of comorbid symptoms of depression

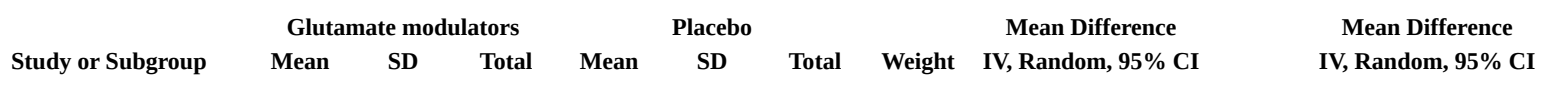

6.3.1 Children's Depression Inventory (CDI)

$\begin{array}{lllllllll}\text { Bloch } 2013 & 10.9 & 1.9 & 20 & 7.8 & 1.9 & 19 & 100.0 \% & 3.10[1.91,4.29] \\ \text { Subtotal (95\% CI) } & & & \mathbf{2 0} & & & \mathbf{1 9} & \mathbf{1 0 0 . 0 \%} & \mathbf{3 . 1 0}[\mathbf{1 . 9 1 , \mathbf { 4 . 2 9 } ]}\end{array}$

Heterogeneity: Not applicable

Test for overall effect: $\mathrm{Z}=5.09(\mathrm{P}<0.00001)$

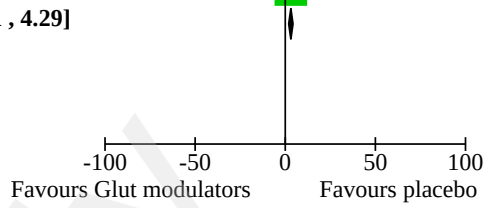

Analysis 6.4. Comparison 6: Glutamate modulators versus placebo in children and adolescents, Outcome 4: Tolerability of treatment - Dropouts due to adverse eventsDropouts due to adverse events

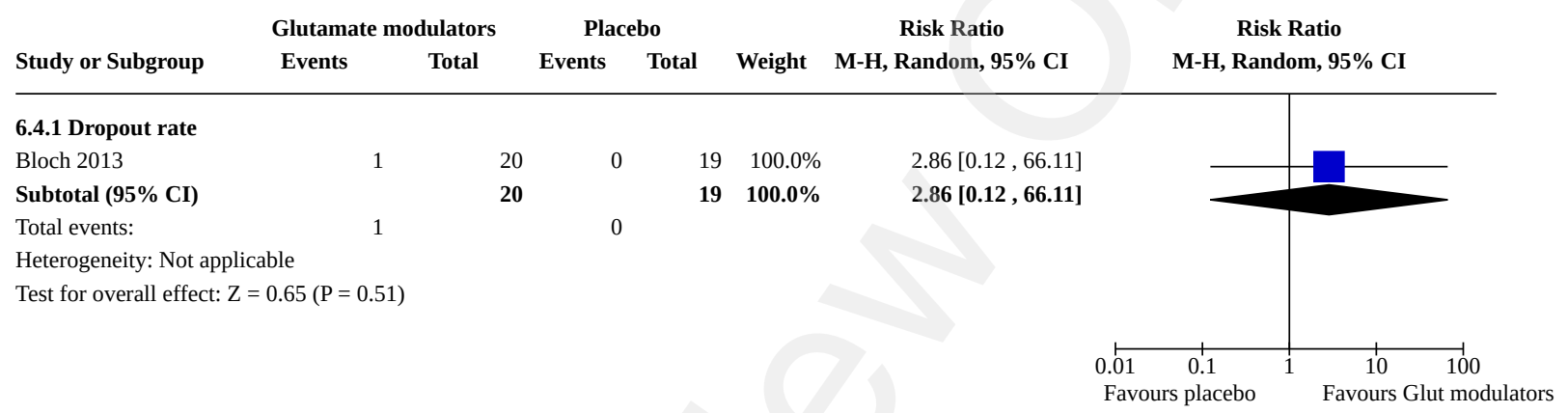

Analysis 6.5. Comparison 6: Glutamate modulators versus placebo in children and adolescents, Outcome 5: Tolerability of treatment - Common adverse events (> 20\%)

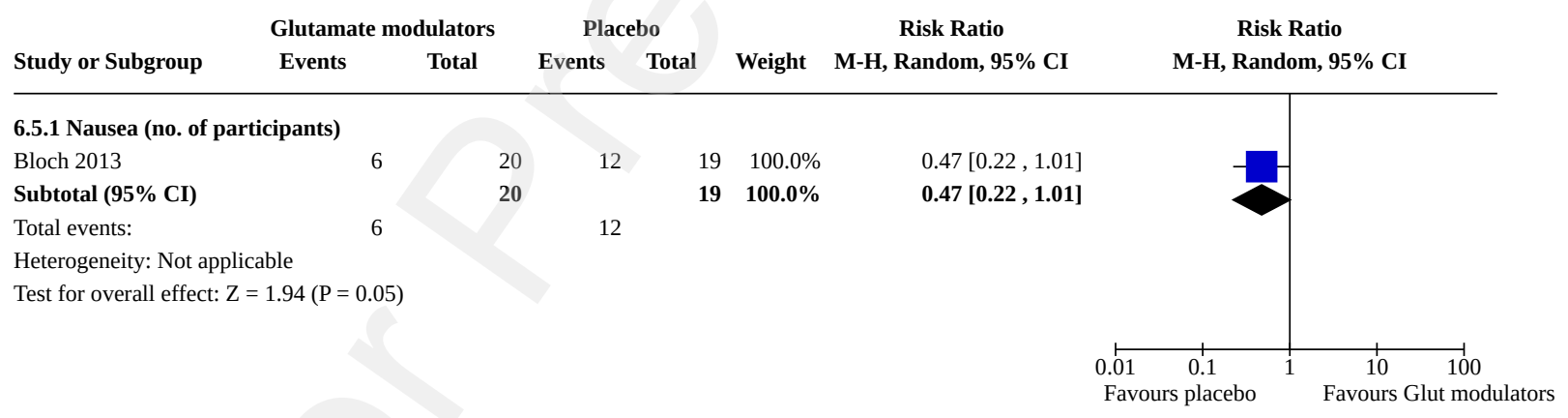

Comparison 7. Opioid antagonists versus placebo in adults

\begin{tabular}{lllll}
\hline Outcome or subgroup title & No. of studies & $\begin{array}{l}\text { No. of partici- } \\
\text { pants }\end{array}$ & Statistical method & Effect size \\
\hline 7.1 Treatment response & 2 & 68 & $\begin{array}{l}\text { Risk Ratio (M-H, Random, } \\
95 \% \mathrm{Cl})\end{array}$ & $2.14[0.25,18.17]$ \\
\hline
\end{tabular}




\begin{tabular}{|c|c|c|c|c|}
\hline Outcome or subgroup title & No. of studies & $\begin{array}{l}\text { No. of partici- } \\
\text { pants }\end{array}$ & Statistical method & Effect size \\
\hline $\begin{array}{l}\text { 7.1.1 Clinical Global Impressions - Im- } \\
\text { provement Scale (CGI-I) (no. of respon- } \\
\text { ders) }\end{array}$ & 1 & 51 & $\begin{array}{l}\text { Risk Ratio (M-H, Random, } \\
95 \% \mathrm{Cl})\end{array}$ & $1.04[0.49,2.19]$ \\
\hline $\begin{array}{l}\text { 7.1.2 NIMH Trichotillomania Severity } \\
\text { Scale (NIMH-TSS) (no. of responders as } \\
>50 \% \text { reduction) }\end{array}$ & 1 & 17 & $\begin{array}{l}\text { Risk Ratio (M-H, Random, } \\
95 \% \mathrm{Cl})\end{array}$ & $\begin{array}{l}9.62[0.57 \\
161.44]\end{array}$ \\
\hline 7.2 Reduction of TTM symptom severity & 1 & & $\begin{array}{l}\text { Mean Difference (IV, Ran- } \\
\text { dom, } 95 \% \mathrm{Cl} \text { ) }\end{array}$ & Subtotals only \\
\hline $\begin{array}{l}\text { 7.2.1 Clinical Global Impression - Severi- } \\
\text { ty Scale (CGI-S) }\end{array}$ & 1 & 51 & $\begin{array}{l}\text { Mean Difference (IV, Ran- } \\
\text { dom, } 95 \% \mathrm{CI} \text { ) }\end{array}$ & $-0.37[-1.10,0.36]$ \\
\hline $\begin{array}{l}\text { 7.2.2 Massachusetts General Hospital } \\
\text { Hairpulling Scale (MGH-HPS) }\end{array}$ & 1 & 51 & $\begin{array}{l}\text { Mean Difference (IV, Ran- } \\
\text { dom, } 95 \% \mathrm{CI} \text { ) }\end{array}$ & $-1.14[-4.18,1.90]$ \\
\hline $\begin{array}{l}\text { 7.2.3 NIMH Trichotillomania Severity } \\
\text { Scale (NIMH-TSS) }\end{array}$ & 1 & 51 & $\begin{array}{l}\text { Mean Difference (IV, Ran- } \\
\text { dom, } 95 \% \mathrm{CI} \text { ) }\end{array}$ & $-0.79[-3.62,2.04]$ \\
\hline $\begin{array}{l}\text { 7.3 Reduction of comorbid symptoms of } \\
\text { depression }\end{array}$ & 1 & & $\begin{array}{l}\text { Mean Difference (IV, Ran- } \\
\text { dom, } 95 \% \mathrm{CI} \text { ) }\end{array}$ & Subtotals only \\
\hline $\begin{array}{l}\text { 7.3.1 Hamilton Depression Rating Scale } \\
\text { (HAM-D) }\end{array}$ & 1 & 51 & $\begin{array}{l}\text { Mean Difference (IV, Ran- } \\
\text { dom, } 95 \% \mathrm{CI} \text { ) }\end{array}$ & $0.66[-1.26,2.58]$ \\
\hline 7.4 Quality of Life - functional disability & 1 & & $\begin{array}{l}\text { Mean Difference (IV, Ran- } \\
\text { dom, } 95 \% \mathrm{Cl} \text { ) }\end{array}$ & Subtotals only \\
\hline 7.4.1 Sheehan Disability Scale (SDS) & 1 & 51 & $\begin{array}{l}\text { Mean Difference (IV, Ran- } \\
\text { dom, } 95 \% \mathrm{Cl} \text { ) }\end{array}$ & $-1.91[-5.82,2.00]$ \\
\hline $\begin{array}{l}\text { 7.5 Tolerability of treatment - Common } \\
\text { adverse events }(>20 \%)\end{array}$ & 1 & & $\begin{array}{l}\text { Risk Ratio (M-H, Random, } \\
95 \% \mathrm{Cl})\end{array}$ & Subtotals only \\
\hline 7.5.1 Sedation (no. of participants) & 1 & 51 & $\begin{array}{l}\text { Risk Ratio (M-H, Random, } \\
95 \% \mathrm{Cl})\end{array}$ & $3.12[0.35,28.03]$ \\
\hline
\end{tabular}


Analysis 7.1. Comparison 7: Opioid antagonists versus placebo in adults, Outcome 1: Treatment response

\begin{tabular}{|c|c|c|c|c|c|c|c|}
\hline \multirow[b]{2}{*}{ Study or Subgroup } & \multicolumn{2}{|c|}{ Opioid antagonists } & \multicolumn{2}{|c|}{ Placebo } & \multicolumn{2}{|r|}{ Risk Ratio } & Risk Ratio \\
\hline & Events & Total & Events & Total & Weight & M-H, Random, 95\% CI & M-H, Random, 95\% CI \\
\hline
\end{tabular}

7.1.1 Clinical Global Impressions - Improvement Scale (CGI-I) (no. of responders)

$\begin{array}{lllllll}\text { Grant } 2014 & 9 & 25 & 9 & 26 & 67.7 \% & 1.04[0.49,2.19]\end{array}$

$\begin{array}{lllll}\text { Subtotal }(95 \% \mathrm{CI}) & 25 & 26 & 67.7 \% & 1.04[0.49,2.19]\end{array}$

Heterogeneity: Not applicable

Test for overall effect: $\mathrm{Z}=0.10(\mathrm{P}=0.92)$

7.1.2 NIMH Trichotillomania Severity Scale (NIMH-TSS) (no. of responders as $>\mathbf{5 0} \%$ reduction)

$\begin{array}{lcccccc}\text { Christenson } 1994 & 3 & 7 & 0 & 10 & 32.3 \% & 9.63[0.57,161.44] \\ \text { Subtotal (95\% CI) } & & \mathbf{7} & & \mathbf{1 0} & \mathbf{3 2 . 3 \%} & \mathbf{9 . 6 3}[\mathbf{0 . 5 7}, \mathbf{1 6 1 . 4 4}] \\ \text { Total events: } & 3 & & 0 & & & \end{array}$

Total events:
Heterogeneity: Not applicable

Test for overall effect: $\mathrm{Z}=1.57(\mathrm{P}=0.12)$
Total (95\% CI)
32
$36 \quad \mathbf{1 0 0 . 0} \%$
$2.14[0.25,18.17]$

Total events:

129

Heterogeneity: $\mathrm{Tau}^{2}=1.62 ; \mathrm{Chi}^{2}=2.46, \mathrm{df}=1(\mathrm{P}=0.12) ; \mathrm{I}^{2}=59 \%$

Test for overall effect: $\mathrm{Z}=0.69(\mathrm{P}=0.49)$

Test for subgroup differences: $\mathrm{Chi}^{2}=2.24, \mathrm{df}=1(\mathrm{P}=0.13), \mathrm{I}^{2}=55.3 \%$

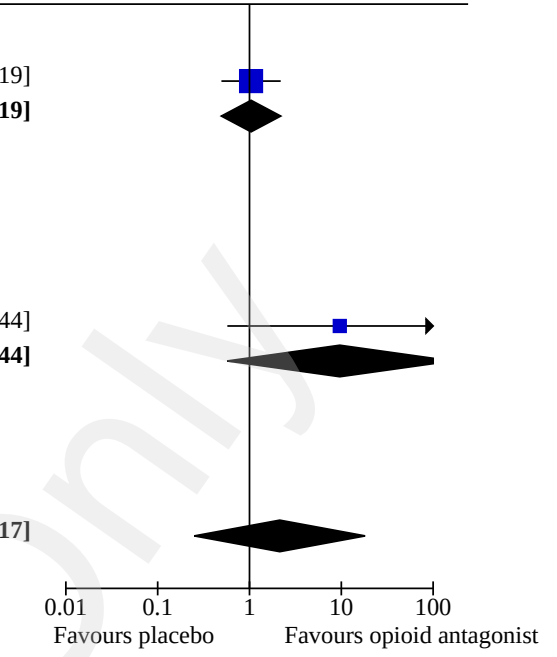

\section{Analysis 7.2. Comparison 7: Opioid antagonists versus placebo in adults, Outcome 2: Reduction of TTM symptom severity}

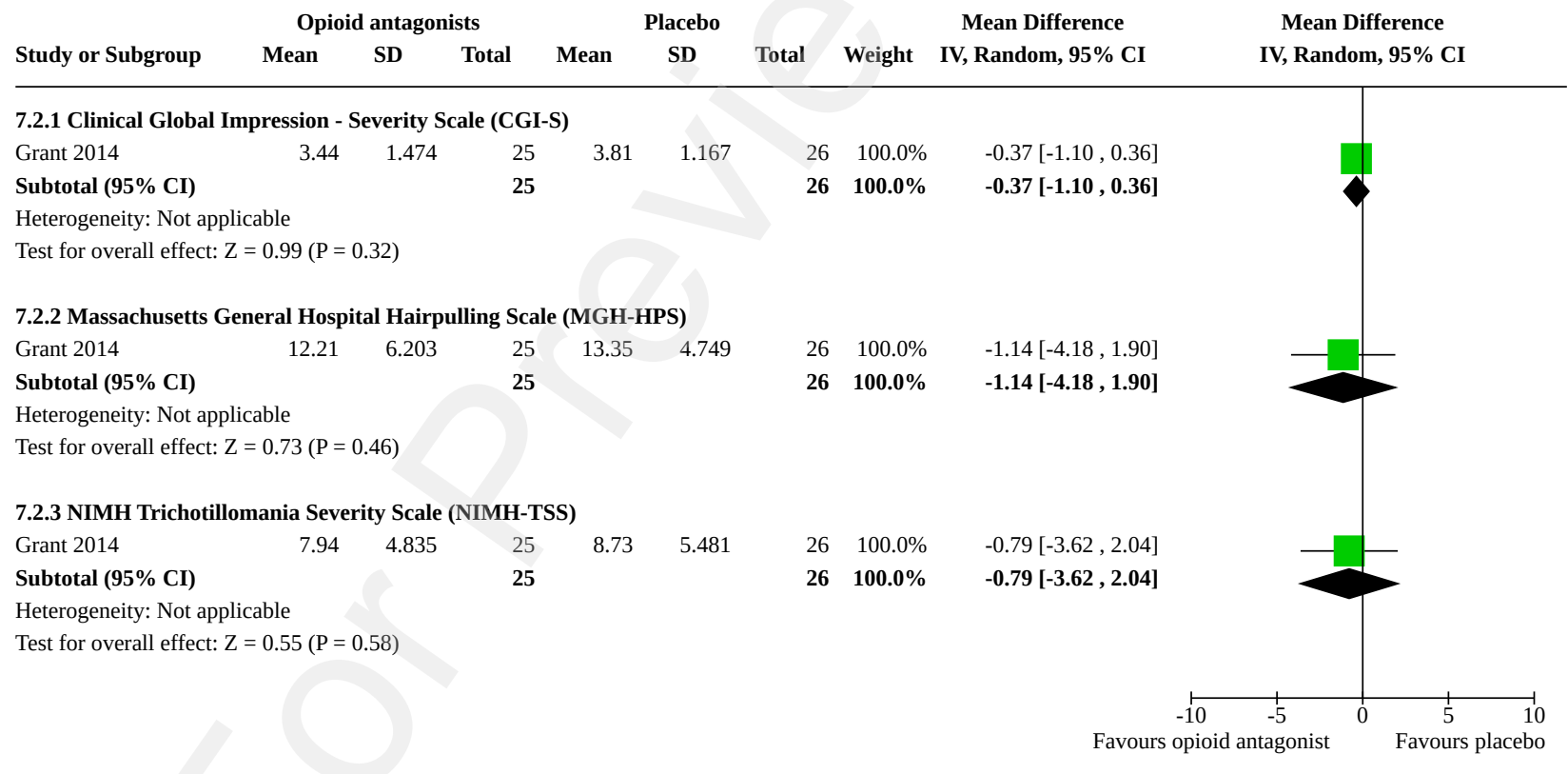




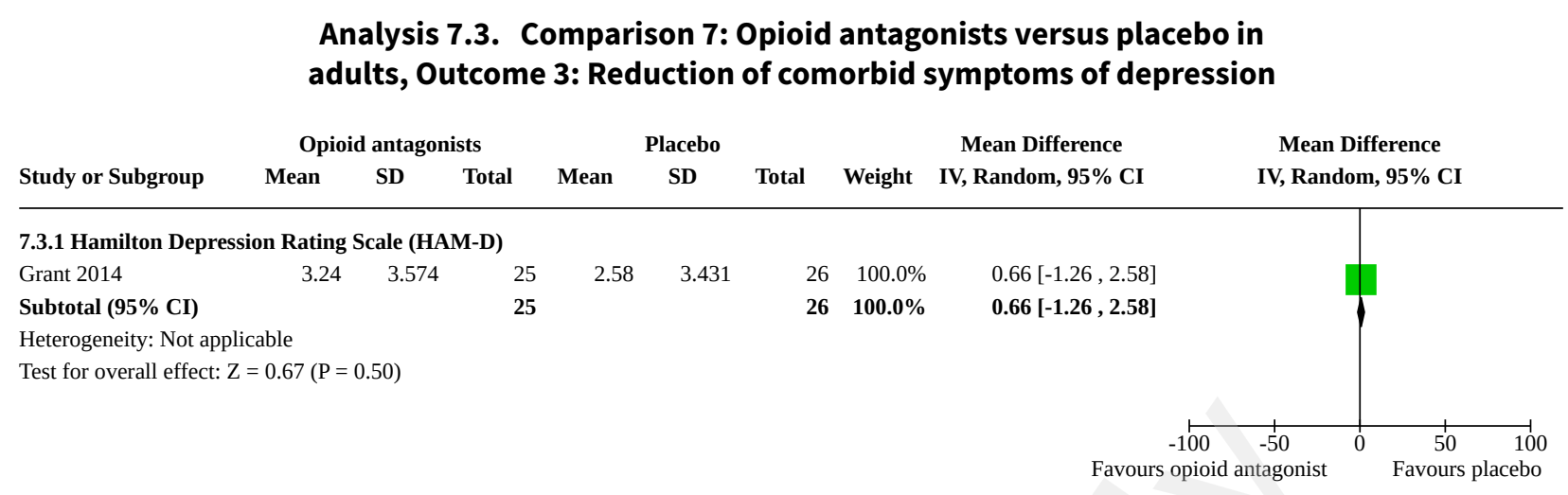

\section{Analysis 7.4. Comparison 7: Opioid antagonists versus placebo in adults, Outcome 4: Quality of Life - functional disability}

\begin{tabular}{|c|c|c|c|c|c|c|c|c|c|}
\hline \multirow[b]{2}{*}{ or Subgroup } & \multicolumn{3}{|c|}{ Opioid antagonists } & \multicolumn{3}{|c|}{ Placebo } & \multicolumn{2}{|r|}{ Mean Difference } & Mean Difference \\
\hline & Mean & SD & Total & Mean & SD & Total & Weight & IV, Random, 95\% CI & IV, Random, 95\% CI \\
\hline
\end{tabular}

7.4.1 Sheehan Disability Scale (SDS)

$\begin{array}{lllllllll}\text { Grant } 2014 & 6.74 & 7.407 & 25 & 8.65 & 6.811 & 26 & 100.0 \% & -1.91[-5.82,2.00]\end{array}$

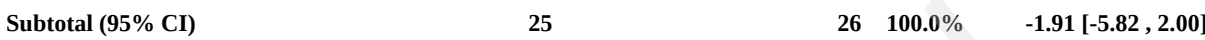

Heterogeneity: Not applicable

Test for overall effect: $\mathrm{Z}=0.96(\mathrm{P}=0.34)$

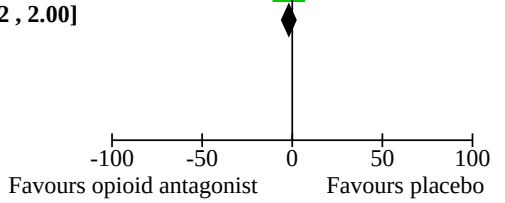

Analysis 7.5. Comparison 7: Opioid antagonists versus placebo in adults, Outcome 5: Tolerability of treatment - Common adverse events (> 20\%)

\begin{tabular}{|c|c|c|c|c|c|c|}
\hline \multirow[b]{2}{*}{ Study or Subgroup } & \multicolumn{2}{|c|}{ Opioid antagonists } & \multicolumn{2}{|c|}{ Placebo } & \multirow[b]{2}{*}{ Weight } & \multirow{2}{*}{$\begin{array}{c}\text { Risk Ratio } \\
\text { M-H, Random, 95\% CI }\end{array}$} \\
\hline & Events & Total & Events & Total & & \\
\hline \multicolumn{7}{|c|}{ 7.5.1 Sedation (no. of participants) } \\
\hline Grant 2014 & & & 1 & 26 & $100.0 \%$ & $3.12[0.35,28.03]$ \\
\hline Subtotal (95\% CI) & & & & 26 & $100.0 \%$ & $3.12[0.35,28.03]$ \\
\hline Total events: & & & 1 & & & \\
\hline \multicolumn{7}{|c|}{ Heterogeneity: Not applicable } \\
\hline Test for overall effect & $1.02(1$ & & & & & \\
\hline
\end{tabular}

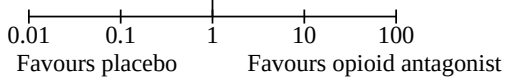

Comparison 8. SSRIs versus placebo in adults

\begin{tabular}{lllll}
\hline Outcome or subgroup title & No. of studies & $\begin{array}{l}\text { No. of partici- } \\
\text { pants }\end{array}$ & Statistical method & Effect size \\
\hline 8.1 Treatment response & 0 & Other data & No numeric data \\
\hline 8.2 Reduction of TTM symptom severity & 1 & $\begin{array}{l}\text { Mean Difference (IV, Ran- } \\
\text { dom, 95\% CI) }\end{array}$ & Subtotals only \\
\hline
\end{tabular}




\begin{tabular}{|c|c|c|c|c|}
\hline Outcome or subgroup title & No. of studies & $\begin{array}{l}\text { No. of partici- } \\
\text { pants }\end{array}$ & Statistical method & Effect size \\
\hline $\begin{array}{l}\text { 8.2.1 Massachusetts General Hospi- } \\
\text { tal Hairpulling Scale (MGH-HPS) mean } \\
\text { change score }\end{array}$ & 1 & 31 & $\begin{array}{l}\text { Mean Difference (IV, Ran- } \\
\text { dom, } 95 \% \mathrm{CI} \text { ) }\end{array}$ & $-0.47[-4.30,3.36]$ \\
\hline $\begin{array}{l}8.3 \text { Reduction of comorbid symptoms of } \\
\text { depression }\end{array}$ & 1 & & $\begin{array}{l}\text { Mean Difference (IV, Ran- } \\
\text { dom, } 95 \% \mathrm{CI} \text { ) }\end{array}$ & Subtotals only \\
\hline $\begin{array}{l}\text { 8.3.1 Hamilton Depression Rating Scale } \\
\text { (HAM-D) }\end{array}$ & 1 & 32 & $\begin{array}{l}\text { Mean Difference (IV, Ran- } \\
\text { dom, } 95 \% \mathrm{CI} \text { ) }\end{array}$ & $0.80[-1.90,3.50]$ \\
\hline $\begin{array}{l}\text { 8.4 Tolerability of treatment - Dropouts } \\
\text { due to adverse events }\end{array}$ & 2 & & $\begin{array}{l}\text { Risk Ratio }(\mathrm{M}-\mathrm{H}, \text { Random, } \\
95 \% \mathrm{Cl})\end{array}$ & Subtotals only \\
\hline 8.4.1 Dropout rate & 2 & 78 & $\begin{array}{l}\text { Risk Ratio (M-H, Random, } \\
95 \% \mathrm{Cl})\end{array}$ & $3.00[0.33,27.62]$ \\
\hline $\begin{array}{l}8.5 \text { Tolerability of treatment - Common } \\
\text { adverse events }(>20 \%)\end{array}$ & 2 & & $\begin{array}{l}\text { Risk Ratio (M-H, Random, } \\
95 \% \mathrm{Cl})\end{array}$ & Subtotals only \\
\hline 8.5.1 Nausea (no. of participants) & 1 & 32 & $\begin{array}{l}\text { Risk Ratio (M-H, Random, } \\
95 \% \mathrm{Cl})\end{array}$ & $2.50[0.57,11.05]$ \\
\hline 8.5.2 Insomnia (no. of participants) & 1 & 32 & $\begin{array}{l}\text { Risk Ratio (M-H, Random, } \\
95 \% \mathrm{Cl})\end{array}$ & $0.50[0.11,2.35]$ \\
\hline $\begin{array}{l}\text { 8.5.3 Central nervous system or psychi- } \\
\text { atric effects (nightmares, insomnia, dizzi- } \\
\text { ness, irritability, anxiety, feeling of doom) } \\
\text { (no. of participants) }\end{array}$ & 1 & 46 & $\begin{array}{l}\text { Risk Ratio (M-H, Random, } \\
95 \% \mathrm{Cl})\end{array}$ & $1.38[1.04,1.83]$ \\
\hline 8.5.4 GIT effects (no. of participants) & 1 & 46 & $\begin{array}{l}\text { Risk Ratio (M-H, Random, } \\
95 \% \mathrm{Cl})\end{array}$ & $4.67[1.55,14.09]$ \\
\hline
\end{tabular}

\section{Analysis 8.1. Comparison 8: SSRIs versus placebo in adults, Outcome 1: Treatment response}

$\begin{array}{ll}\text { Study } & \text { Heading } 1\end{array}$

Analysis 8.2. Comparison 8: SSRIs versus placebo in adults, Outcome 2: Reduction of TTM symptom severity

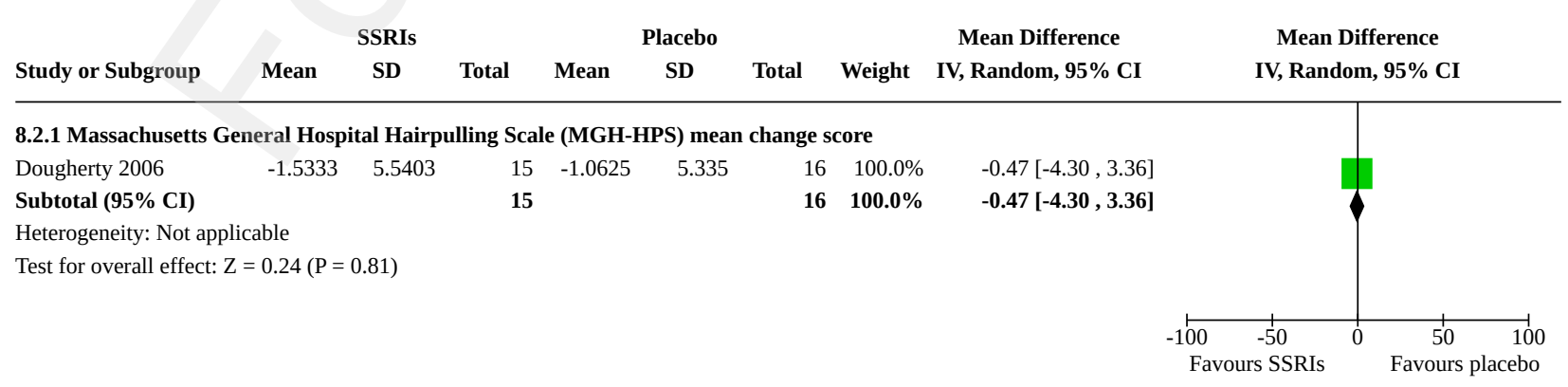


Analysis 8.3. Comparison 8: SSRIs versus placebo in adults, Outcome 3: Reduction of comorbid symptoms of depression

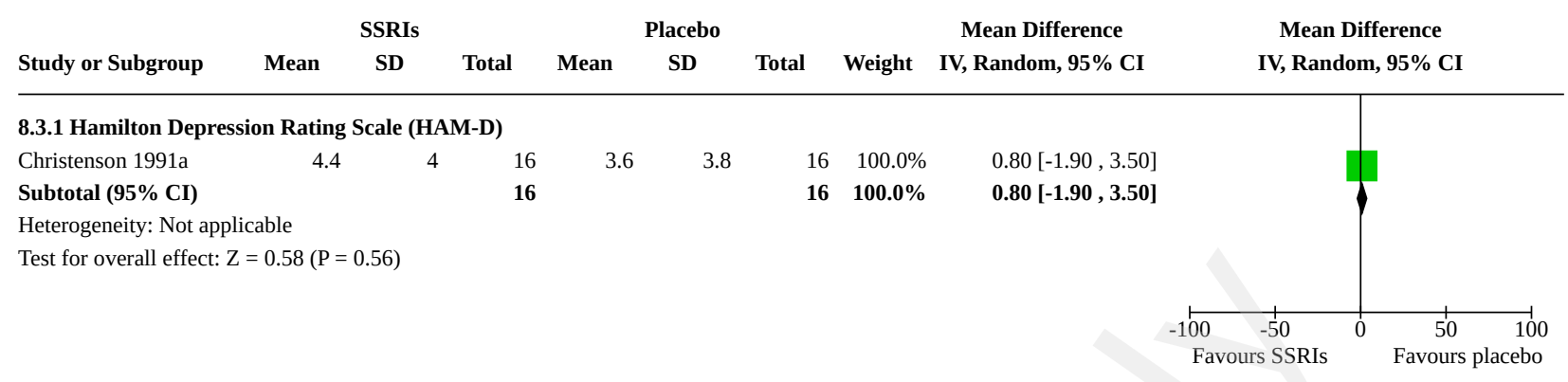

Analysis 8.4. Comparison 8: SSRIs versus placebo in adults, Outcome

4: Tolerability of treatment - Dropouts due to adverse events

SSRIs

Study or Subgroup
Placebo Event
Risk Ratio

Weight $\quad$ M-H, Random, 95\% CI
Risk Ratio M-H, Random, 95\% CI

\subsubsection{Dropout rate}

Christenson 1991a

Streichenwein 1995

Subtotal (95\% CI)

Total events:

\section{6}

23

39

2

Heterogeneity: $\mathrm{Tau}^{2}=0.00 ; \mathrm{Chi}^{2}=0.00, \mathrm{df}=1(\mathrm{P}=1.00) ; \mathrm{I}^{2}=0 \%$

Test for overall effect: $\mathrm{Z}=0.97(\mathrm{P}=0.33)$
$3.00[0.13,68.57]$

$3.00[0.13,70.02]$

$3.00[0.33,27.62]$

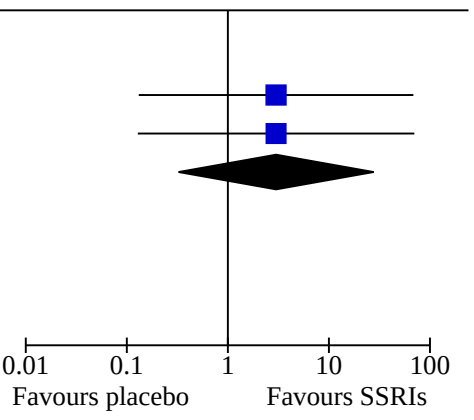




\section{Analysis 8.5. Comparison 8: SSRIs versus placebo in adults, Outcome} 5: Tolerability of treatment - Common adverse events ( $>20 \%$ )

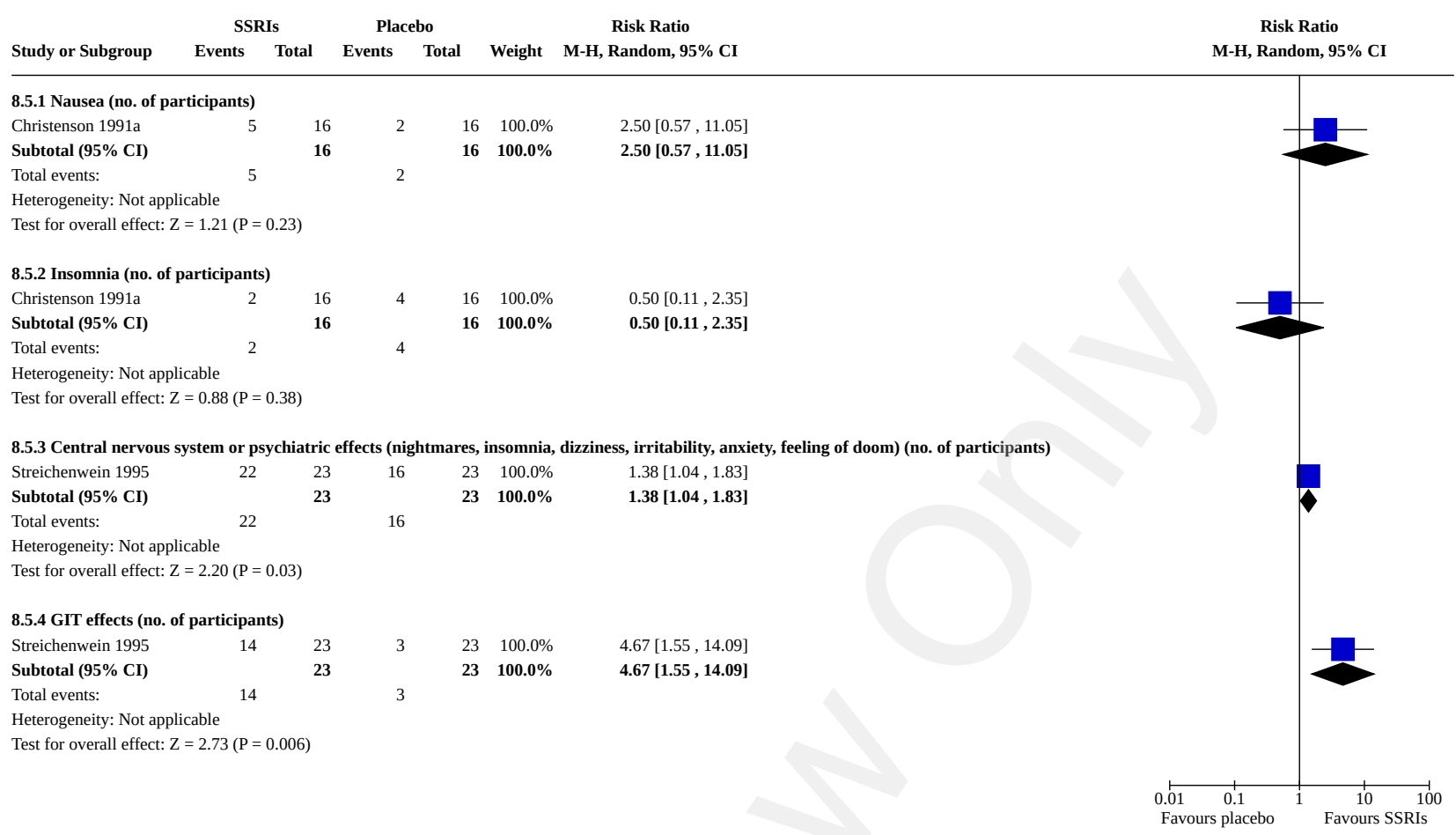

\section{Comparison 9. TCAs with predominantly SRI actions versus placebo in adults}

\begin{tabular}{|c|c|c|c|c|}
\hline Outcome or subgroup title & No. of studies & $\begin{array}{l}\text { No. of partici- } \\
\text { pants }\end{array}$ & Statistical method & Effect size \\
\hline 9.1 Treatment response & 1 & & $\begin{array}{l}\text { Risk Ratio (M-H, Random, } \\
95 \% \mathrm{Cl})\end{array}$ & Subtotals only \\
\hline $\begin{array}{l}\text { 9.1.1 Clinical Global Impressions - Im- } \\
\text { provement Scale (CGI-I) (no. of respon- } \\
\text { ders) }\end{array}$ & 1 & 16 & $\begin{array}{l}\text { Risk Ratio (M-H, Random, } \\
95 \% \mathrm{Cl})\end{array}$ & $5.73[0.36,90.83]$ \\
\hline $\begin{array}{l}9.2 \text { Reduction of TTM symptom severi- } \\
\text { ty }\end{array}$ & 1 & & $\begin{array}{l}\text { Mean Difference (IV, Ran- } \\
\text { dom, } 95 \% \mathrm{CI} \text { ) }\end{array}$ & Subtotals only \\
\hline $\begin{array}{l}\text { 9.2.1 NIMH Trichotillomania Impair- } \\
\text { ment Scale (NIMH-TIS) }\end{array}$ & 1 & 16 & $\begin{array}{l}\text { Mean Difference (IV, Ran- } \\
\text { dom, } 95 \% \mathrm{CI} \text { ) }\end{array}$ & $1.60[-0.82,4.02]$ \\
\hline $\begin{array}{l}\text { 9.2.2 NIMH Trichotillomania Severity } \\
\text { Scale (NIMH-TSS) }\end{array}$ & 1 & 16 & $\begin{array}{l}\text { Mean Difference (IV, Ran- } \\
\text { dom, } 95 \% \mathrm{CI} \text { ) }\end{array}$ & $-3.00[-6.52,0.52]$ \\
\hline $\begin{array}{l}\text { 9.3 Tolerability of treatment - Dropouts } \\
\text { due to adverse eventsDropouts due to } \\
\text { adverse events }\end{array}$ & 1 & & $\begin{array}{l}\text { Risk Ratio (M-H, Random, } \\
95 \% \mathrm{Cl})\end{array}$ & Subtotals only \\
\hline 9.3.1 Dropout rate & 1 & 16 & $\begin{array}{l}\text { Risk Ratio (M-H, Random, } \\
95 \% \mathrm{Cl})\end{array}$ & $4.45[0.27,73.81]$ \\
\hline
\end{tabular}




\begin{tabular}{|c|c|c|c|c|}
\hline Outcome or subgroup title & No. of studies & $\begin{array}{l}\text { No. of partici- } \\
\text { pants }\end{array}$ & Statistical method & Effect size \\
\hline $\begin{array}{l}\text { 9.4 Tolerability of treatment - Common } \\
\text { adverse events }(>20 \%)\end{array}$ & 1 & & $\begin{array}{l}\text { Risk Ratio (M-H, Random, } \\
95 \% \mathrm{Cl} \text { ) }\end{array}$ & Subtotals only \\
\hline 9.4.1 Tremor (no. of participants) & 1 & 16 & $\begin{array}{l}\text { Risk Ratio (M-H, Random, } \\
95 \% \mathrm{Cl} \text { ) }\end{array}$ & $4.45[0.27,73.81]$ \\
\hline 9.4.2 Sedation (no. of participants) & 1 & 16 & $\begin{array}{l}\text { Risk Ratio (M-H, Random, } \\
95 \% \mathrm{Cl} \text { ) }\end{array}$ & $3.18[0.18,56.95]$ \\
\hline 9.4.3 Dry mouth (no. of participants) & 1 & 16 & $\begin{array}{l}\text { Risk Ratio (M-H, Random, } \\
95 \% \mathrm{Cl} \text { ) }\end{array}$ & $3.18[0.18,56.95]$ \\
\hline 9.4.4 Constipation (no. of participants) & 1 & 16 & $\begin{array}{l}\text { Risk Ratio (M-H, Random, } \\
95 \% \mathrm{Cl} \text { ) }\end{array}$ & $3.18[0.18,56.95]$ \\
\hline
\end{tabular}

Analysis 9.1. Comparison 9: TCAs with predominantly SRI actions versus placebo in adults, Outcome 1: Treatment response

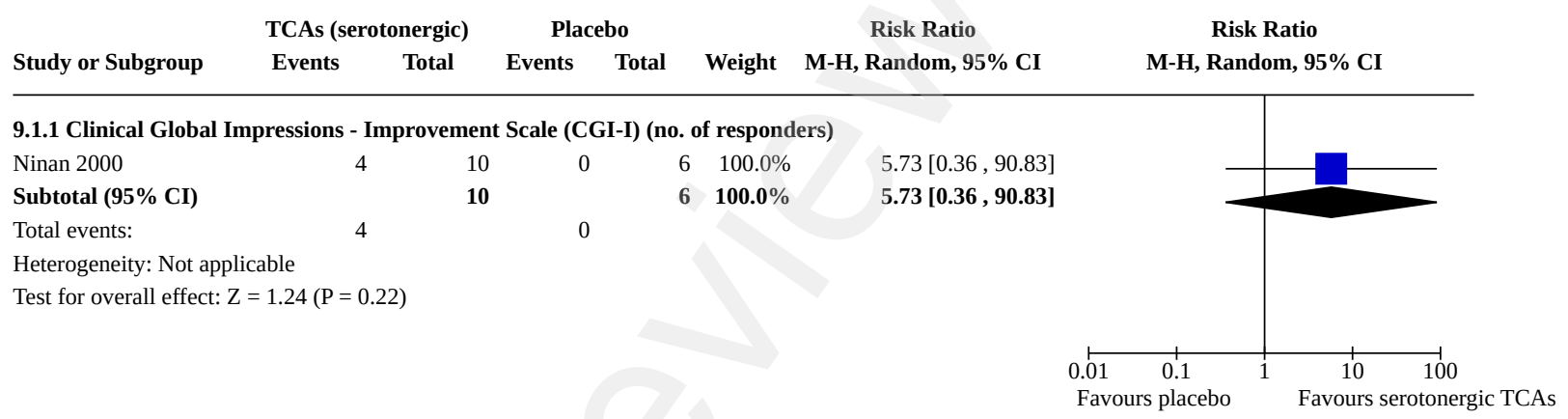

\section{Analysis 9.2. Comparison 9: TCAs with predominantly SRI actions versus placebo in adults, Outcome 2: Reduction of TTM symptom severity}

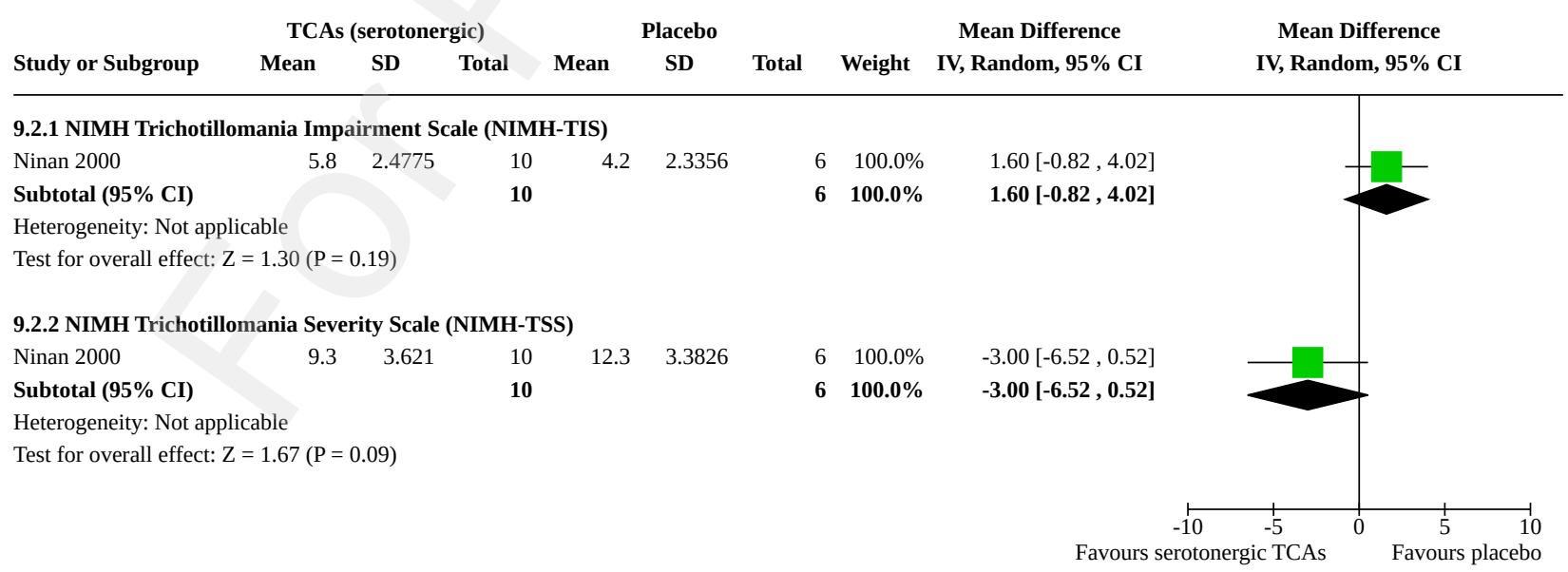


Analysis 9.3. Comparison 9: TCAs with predominantly SRI actions versus placebo in adults, Outcome 3: Tolerability of treatment - Dropouts due to adverse eventsDropouts due to adverse events

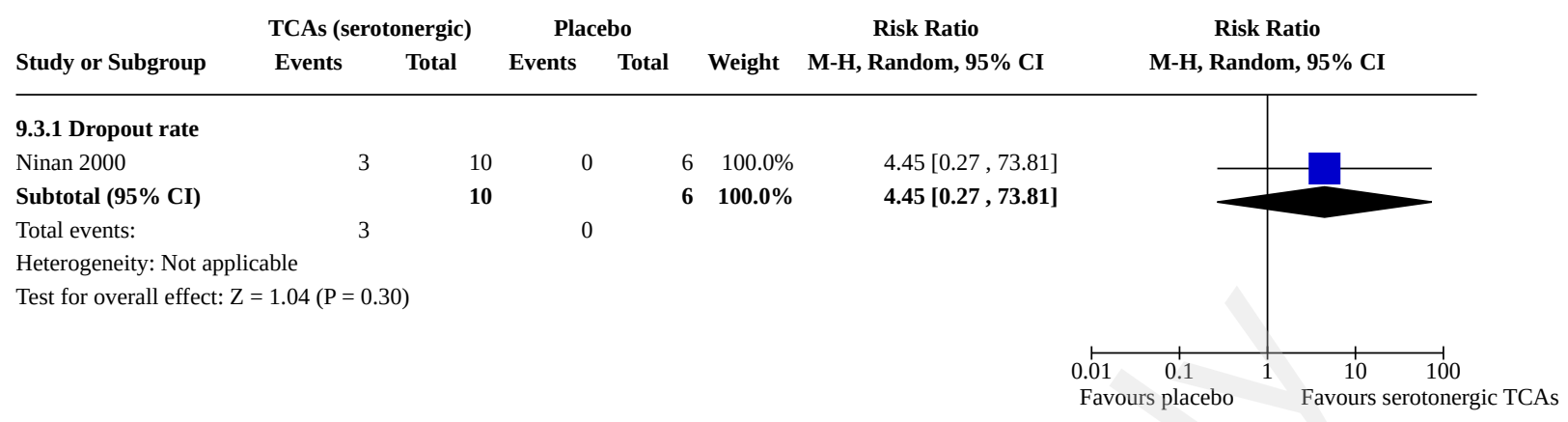

Analysis 9.4. Comparison 9: TCAs with predominantly SRI actions versus placebo in adults, Outcome 4: Tolerability of treatment - Common adverse events $(>20 \%)$

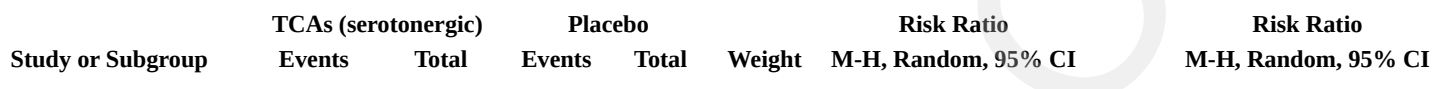

9.4.1 Tremor (no. of participants)

Subtotal (95\% CI)

$\begin{array}{llll}10 & 0 & 6 & 100.0 \%\end{array}$

$4.45[0.27,73.81]$

Total events:

$6 \quad \mathbf{1 0 0 . 0} \%$

$4.45[0.27,73.81]$

Heterogeneity: Not applicable

Test for overall effect: $\mathrm{Z}=1.04(\mathrm{P}=0.30)$

9.4.2 Sedation (no. of participants)

Ninan 2000

Subtotal (95\% CI)

2

Total events:

210

10

$6 \quad 100.0 \%$

$3.18[0.18,56.95]$

Heterogeneity: Not applicable

Test for overall effect: $\mathrm{Z}=0.79(\mathrm{P}=0.43)$

9.4.3 Dry mouth (no. of participants)

Ninan 2000

2

Subtotal (95\% CI)

Total events:

H, Random, 95\% CI

Heterogeneity: Not applicable

Test for overall effect: $\mathrm{Z}=0.79(\mathrm{P}=0.43)$

9.4.4 Constipation (no. of participants)

Ninan $2000 \quad 2$

Subtotal (95\% CI)

10

$6 \quad \mathbf{1 0 0 . 0} \%$

$3.18[0.18,56.95]$

Total events:

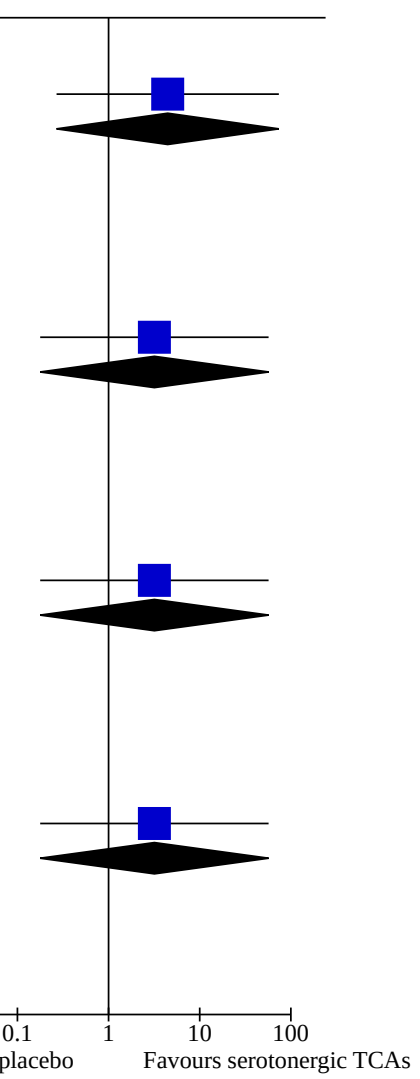

Comparison 10. TCAs with predominantly SRI actions versus other TCAs in adults

\begin{tabular}{lllll}
\hline Outcome or subgroup title & No. of studies & $\begin{array}{l}\text { No. of partici- } \\
\text { pants }\end{array}$ & Statistical method & Effect size \\
\hline 10.1 Treatment response & 1 & $\begin{array}{l}\text { Mean Difference (IV, Random, } \\
95 \% \mathrm{Cl})\end{array}$ & Subtotals only \\
\hline
\end{tabular}




\begin{tabular}{|c|c|c|c|c|}
\hline Outcome or subgroup title & No. of studies & $\begin{array}{l}\text { No. of partici- } \\
\text { pants }\end{array}$ & Statistical method & Effect size \\
\hline $\begin{array}{l}\text { 10.1.1 Physician-rated Clinical } \\
\text { Progress Scale }\end{array}$ & 1 & 26 & $\begin{array}{l}\text { Mean Difference (IV, Random, } \\
95 \% \mathrm{CI} \text { ) }\end{array}$ & $\begin{array}{l}-4.00[-6.13 \\
-1.87]\end{array}$ \\
\hline $\begin{array}{l}\text { 10.2 Reduction of TTM symptom sever- } \\
\text { ity }\end{array}$ & 1 & & $\begin{array}{l}\text { Mean Difference (IV, Random, } \\
95 \% \mathrm{CI} \text { ) }\end{array}$ & Subtotals only \\
\hline $\begin{array}{l}\text { 10.2.1 NIMH Trichotillomania Impair- } \\
\text { ment Scale (NIMH-TIS) }\end{array}$ & 1 & 26 & $\begin{array}{l}\text { Mean Difference (IV, Random, } \\
95 \% \mathrm{CI})\end{array}$ & $\begin{array}{l}-2.00[-3.58 \\
-0.42]\end{array}$ \\
\hline $\begin{array}{l}\text { 10.2.2 NIMH Trichotillomania Severity } \\
\text { Scale (NIMH-TSS) }\end{array}$ & 1 & 26 & $\begin{array}{l}\text { Mean Difference (IV, Random, } \\
95 \% \mathrm{CI} \text { ) }\end{array}$ & $-3.80[-8.08,0.48]$ \\
\hline $\begin{array}{l}10.3 \text { Reduction of comorbid symptoms } \\
\text { of depression }\end{array}$ & 1 & & $\begin{array}{l}\text { Mean Difference (IV, Random, } \\
95 \% \mathrm{CI} \text { ) }\end{array}$ & Subtotals only \\
\hline $\begin{array}{l}\text { 10.3.1 NIMH Global Assessment Scale } \\
\text { for Depression (NIMH-GASD) }\end{array}$ & 1 & 26 & $\begin{array}{l}\text { Mean Difference (IV, Random, } \\
95 \% \mathrm{CI} \text { ) }\end{array}$ & $-0.70[-1.60,0.20]$ \\
\hline $\begin{array}{l}\text { 10.4 Tolerability of treatment - } \\
\text { Dropouts due to adverse events }\end{array}$ & 1 & & $\begin{array}{l}\text { Risk Ratio (M-H, Random, } \\
95 \% \mathrm{Cl})\end{array}$ & Subtotals only \\
\hline 10.4.1 Dropout rate & 1 & 26 & $\begin{array}{l}\text { Risk Ratio (M-H, Random, } \\
95 \% \mathrm{Cl})\end{array}$ & Not estimable \\
\hline $\begin{array}{l}\text { 10.5 Tolerability of treatment - Com- } \\
\text { mon adverse events }(>20 \%)\end{array}$ & 1 & & $\begin{array}{l}\text { Risk Ratio (M-H, Random, } \\
95 \% \mathrm{Cl})\end{array}$ & Subtotals only \\
\hline $\begin{array}{l}\text { 10.5.1 Constipation (no. of partici- } \\
\text { pants) }\end{array}$ & 1 & 26 & $\begin{array}{l}\text { Risk Ratio (M-H, Random, } \\
95 \% \mathrm{Cl})\end{array}$ & $0.83[0.34,2.06]$ \\
\hline 10.5.2 Dry mouth (no. of participants) & 1 & 26 & $\begin{array}{l}\text { Risk Ratio (M-H, Random, } \\
95 \% \mathrm{Cl} \text { ) }\end{array}$ & $0.62[0.28,1.41]$ \\
\hline 10.5.3 Tremor (no. of participants) & 1 & 26 & $\begin{array}{l}\text { Risk Ratio (M-H, Random, } \\
95 \% \mathrm{Cl} \text { ) }\end{array}$ & $2.33[0.77,7.10]$ \\
\hline
\end{tabular}

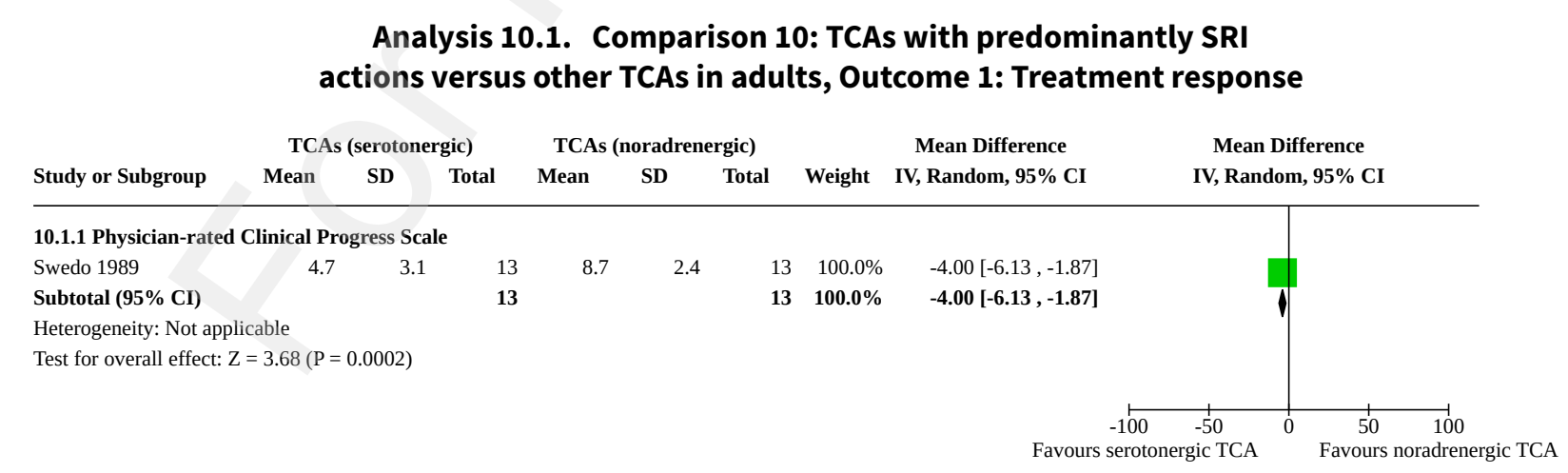




\section{Analysis 10.2. Comparison 10: TCAs with predominantly SRI actions versus} other TCAs in adults, Outcome 2: Reduction of TTM symptom severity

\begin{tabular}{lccccccccc} 
& \multicolumn{4}{c}{ TCAs (serotonergic) } & \multicolumn{2}{c}{ TCAs (noradrenergic) } & & Mean Difference & Mean Difference \\
Study or Subgroup & Mean & SD & Total & Mean & SD & Total & Weight & IV, Random, 95\% CI & IV, Random, 95\% CI
\end{tabular}

10.2.1 NIMH Trichotillomania Impairment Scale (NIMH-TIS)

$\begin{array}{lllllllll}\text { Swedo } 1989 & 4.2 & 2.7 & 13 & 6.2 & 1.1 & 13 & 100.0 \% & -2.00[-3.58,-0.42]\end{array}$

Subtotal (95\% CI)

$\begin{array}{lll}4.2 & 2.7 & 13 \\ - & -2 & \end{array}$

$13 \quad 100.0 \%$

$-2.00[-3.58,-0.42]$

Heterogeneity: Not applicable

Test for overall effect: $\mathrm{Z}=2.47(\mathrm{P}=0.01)$

10.2.2 NIMH Trichotillomania Severity Scale (NIMH-TSS)

$\begin{array}{lllllllll}\text { Swedo } 1989 & 10.6 & 6.4 & 13 & 14.4 & 4.6 & 13 & 100.0 \% & -3.80[-8.08,0.48] \\ \text { Subtotal }(\mathbf{9 5} \% \mathbf{C I}) & & & \mathbf{1 3} & & & \mathbf{1 3} & \mathbf{1 0 0 . 0} \% & \mathbf{- 3 . 8 0}[\mathbf{- 8 . 0 8 , 0 . 4 8}]\end{array}$

Heterogeneity: Not applicable

Test for overall effect: $\mathrm{Z}=1.74(\mathrm{P}=0.08)$

Analysis 10.3. Comparison 10: TCAs with predominantly SRI actions versus other TCAs in adults, Outcome 3: Reduction of comorbid symptoms of depression

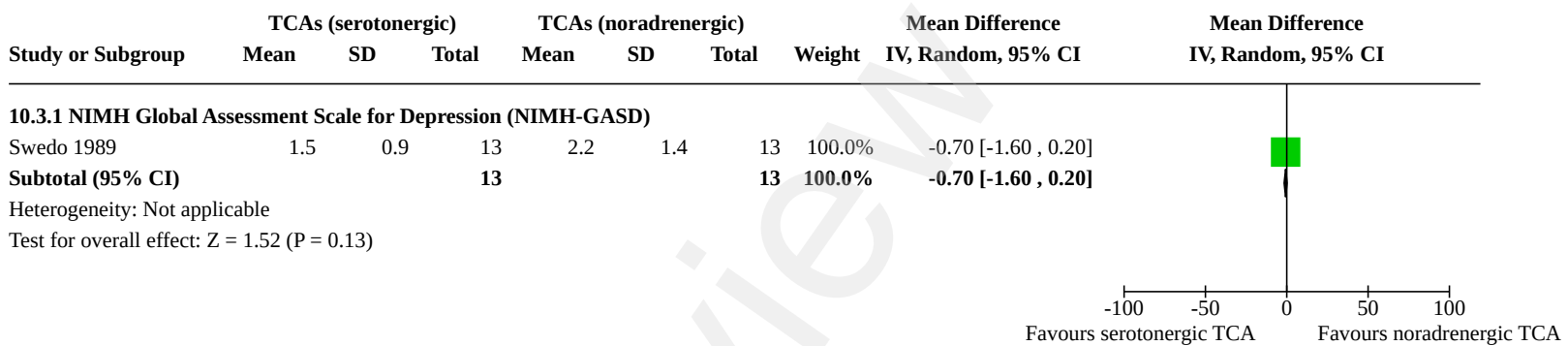

Analysis 10.4. Comparison 10: TCAs with predominantly SRI actions versus other TCAs in adults, Outcome 4: Tolerability of treatment - Dropouts due to adverse events

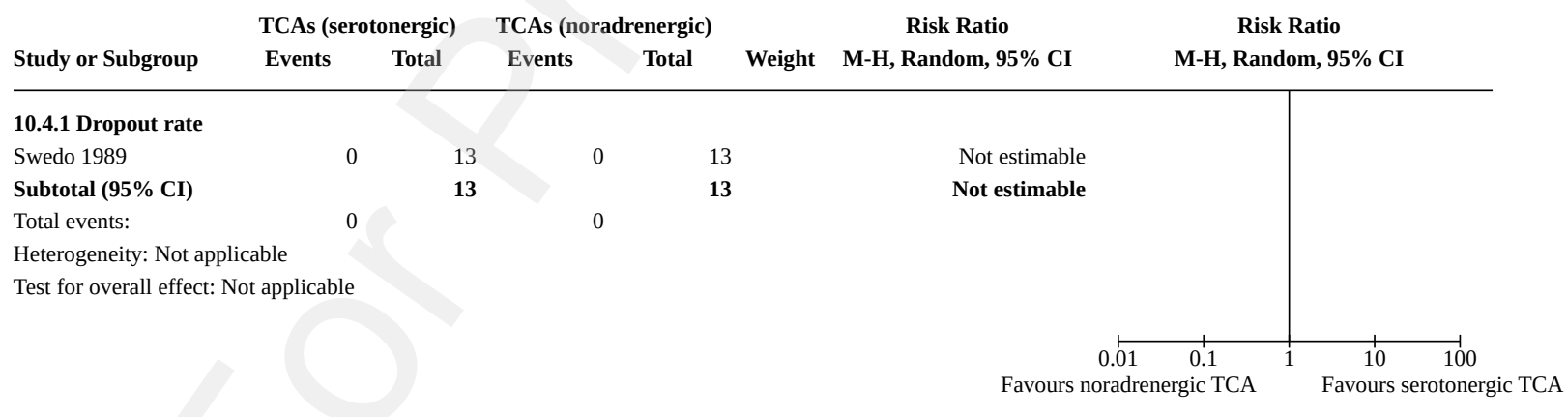


Analysis 10.5. Comparison 10: TCAs with predominantly SRI actions versus other TCAs in adults, Outcome 5: Tolerability of treatment - Common adverse events (>20\%)

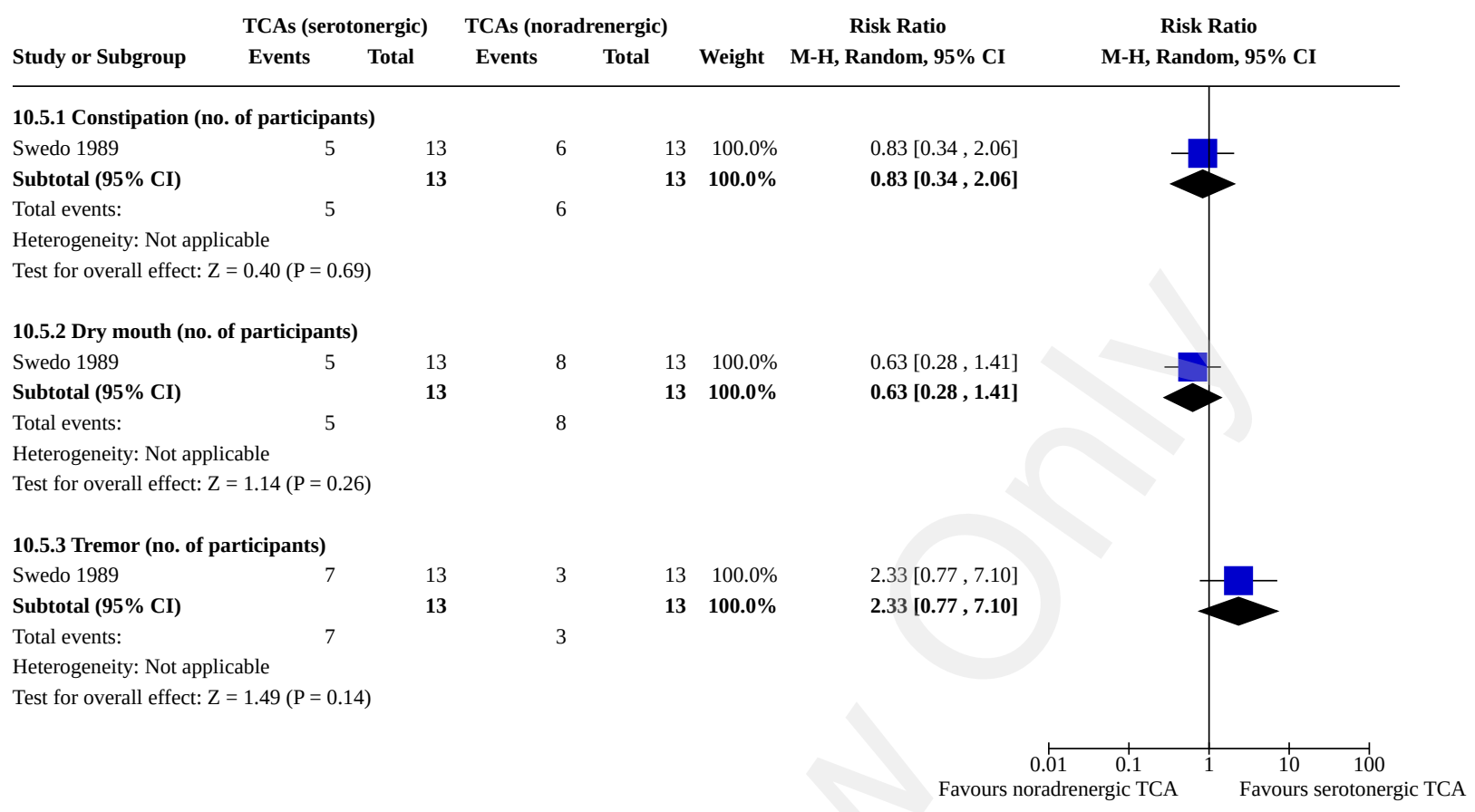

\section{APPENDICES}

\section{Appendix 1. Database search strategies (November 2020)}

Cochrane Common Mental Disorders Controlled Trials Register (CCMDCTR) (all years to June 2016)

trichotillo* OR ((hair adj3 pull*) AND (BFRB or chronic or compulsi* or habit ${ }^{\star}$ or obsess ${ }^{\star}$ or pathologic ${ }^{\star}$ or repeat ${ }^{\star}$ or repetiti $)$ ) AND INREGISTER

Cochrane Central Register of Controlled Trials (CENTRAL; 2020, Issue 11)

trichotillo* OR ((hair adj3 pull $\left.{ }^{\star}\right)$ AND (BFRB or chronic or compulsi ${ }^{\star}$ or habit ${ }^{\star}$ or obsess ${ }^{\star}$ or pathologic ${ }^{\star}$ or repeat ${ }^{\star}$ or repetiti $\left.^{\star}\right)$ )

3. Ovid MEDLINE(R) and Epub Ahead of Print, In-Process \& Other Non-Indexed Citations and Daily <1946 to 26 November 2020> Search Strategy:

\section{Trichotillomania/}

2 trichotillo*.mp.

3 (hair adj3 pull* adj3 (BFRB or chronic or compulsi* or disorder? or habit* or obsess ${ }^{\star}$ or pathologic ${ }^{\star}$ or repeat* or repetiti $\left.{ }^{\star}\right)$ ).mp.

4 or $/ 1-3$

5 controlled clinical trial.pt.

6 randomized controlled trial.pt.

7 clinical trials as topic/

8 (randomi\#ed or randomi\#ation or randomi\#ing).ti,ab,kf.

9 (RCT or "at random" or (random* adj3 (administ* or allocat* or assign* or class* or cluster or crossover or cross-over or control ${ }^{\star}$ or determine $^{\star}$ or divide* or division or distribut ${ }^{\star}$ or expose* or fashion or number ${ }^{\star}$ or place ${ }^{\star}$ or pragmatic or quasi or recruit* or split or subsitut $^{\star}$ or treat $\left.\left.\left.{ }^{\star}\right)\right)\right) . t i, a b, k f$.

10 placebo.ab,ti,kf.

11 trial.ti.

12 (control* $^{*}$ adj3 group $\left.{ }^{\star}\right) \cdot$ ab.

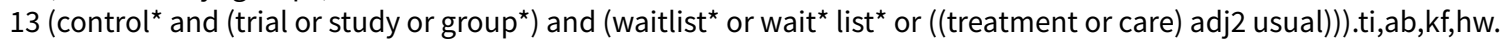


14 ((single or double or triple or treble) adj2 (blind* or mask* or dummy)).ti,ab,kf.

15 double-blind method/ or random allocation/ or single-blind method/

16 or/5-15

17 exp animals/ not humans.sh.

$18(16$ not 17$)$

19 (4 and 18)

4. Ovid PsycINFO <1806 to 26 November 2020>

Search Strategy:

1 trichotillomania/

2 trichotillo*.ti,ab,id.

3 (hair adj3 pull adj3 (BFRB or chronic or compulsi $^{\star}$ or disorder? or habit* or obsess ${ }^{\star}$ or pathologic ${ }^{\star}$ or repeat* or repetiti*)).ti,ab,id.

4 or/ $1-3$

5 clinical trials.sh.

6 (randomi\#ed or randomi\#ation or randomi\#ing).ti,ab,id.

7 (RCT or at random or (random* adj3 (administ* or allocat ${ }^{\star}$ or assign* or class* or control ${ }^{\star}$ or crossover or cross-over or determine ${ }^{\star}$ or divide $^{\star}$ or division or distribut ${ }^{\star}$ or expose ${ }^{\star}$ or fashion or number ${ }^{\star}$ or place or recruit $^{\star}$ or split or subsitut ${ }^{\star}$ or treat $\left.\left.^{\star}\right)\right)$ ).ti,ab,id.

8 (control ${ }^{\star}$ and (trial or study or group) and (placebo or waitlist ${ }^{\star}$ or wait $^{\star}$ list $^{\star}$ or ((treatment or care) adj2 usual))).ti,ab,id,hw.

9 ((single or double or triple or treble) adj2 (blind* or mask* or dummy)).ti,ab,id.

10 trial.ti.

11 placebo.ti,ab,id,hw.

12 treatment outcome.md.

13 treatment effectiveness evaluation.sh.

14 mental health program evaluation.sh.

15 or/5-14

16 (4 and 15)

5. Ovid Embase < 1974 to 26 November 2020>

Search Strategy:

1 trichotillomania/

2 trichotillo ${ }^{\star} . t i, a b, k w$.

3 (hair adj3 pull* adj3 (BFRB or chronic or compulsi* or disorder? or habit* or obsess ${ }^{\star}$ or pathologic ${ }^{\star}$ or repeat* or repetiti ${ }^{\star}$ ).ti,ab,kw.

4 or/ $1-3$

5 randomized controlled trial/

6 randomization.de.

7 controlled clinical trial/ and (Disease Management or Drug Therapy or Prevention or Rehabilitation or Therapy).fs.

8 *clinical trial/

9 placebo.de.

10 placebo.ti,ab.

11 trial.ti.

12 (randomi\#ed or randomi\#ation or randomi\#ing).ti,ab,kw.

13 (RCT or "at random" or (random* adj3 (administ* or allocat* or assign* or class* or cluster or control* or crossover or cross-over or determine $^{\star}$ or divide or division or distribut ${ }^{\star}$ or expose ${ }^{\star}$ or fashion or number ${ }^{\star}$ or place* or pragmatic or quasi or recruit ${ }^{\star}$ or split or subsitut* or treat*))).ti,ab,kw.

14 ((singl\$ or doubl\$ or trebl\$ or tripl\$) adj3 (blind\$ or mask\$ or dummy)).mp.

15 (control ${ }^{\star}$ and (study or group?) and (waitlist* or wait $^{\star}$ list* or ((treatment or care) adj2 usual))).ti,ab,kw,hw.

16 or/5-15

17 ((animal or nonhuman) not (human and (animal or nonhuman))).de. (5546742)

18 (16 not 17$)$

19 (4 and 18)

International Trial Registries

ClinicalTrials.gov (searched 26 November 2020)

(trichotillomania or hair pulling) 
WHO-ICTRP (searched 2 December 2019)

(trichotillo*)

\section{Thesis Databases}

Proquest Dissertations \& Theses Global (searched 26 November 2020)

noft(trichotillo* or "hair pulling") AND noft((RCT OR randomized OR randomised OR randomly OR assignment OR allocation) )

Open Access Theses and Dissertations (oatd.org) (searched 2 December 2019)

(trichotillomania or "hair pulling") AND (RCT OR randomized OR randomised OR randomly)

DART - Europe e-theses Portal (dart-europe.eu) (searched 2 December 2019)

(trichotillomania or "hair pulling") AND (RCT OR randomized OR randomised OR randomly)

Networked Digital Library of Theses and Dissertations (ndltd.org) (searched 2 December 2019)

(trichotillomania or "hair pulling") AND (RCT OR randomized OR randomised OR randomly)

\section{Appendix 2. Database search strategies (July 2013)}

Cochrane Common Mental Disorders Controlled Trials Register (CCMDCTR)

The CCMDCTR-Studies Register was searched using the following controlled vocabulary terms:

Condition = trichotillomania OR "pathological hair pulling"

The CCMDCTR-References Register was searched to retrieve additional untagged references using the free-text term: "trichotillomani`"

Cochrane Central Register of Controlled Trials (CENTRAL; 2013, Issue 7)

"trichotillomania"

MEDLINE (1966-2013) - via PubMed was searched using the following terms:

(randomised controlled trial [pt] OR controlled clinical trial [pt] OR randomised controlled trials [mh] OR random allocation [mh] OR double-blind method [mh] OR single-blind method [mh] OR clinical trial [pt] OR clinical trials [mh] OR ("clinical trial" [tw]) OR ((singl* [tw] OR doubl* [tw] OR trebl* [tw] OR tripl* [tw]) AND (mask* [tw] OR blind* [tw])) OR ("latin square" [tw]) OR placebos [mh] OR placebo* [tw] OR random* [tw] OR research design [mh:noexp] OR comparative study [mh] OR evaluation studies [mh] OR follow-up studies [mh] OR prospective studies [mh] OR cross-over studies [mh] OR control ${ }^{\star}$ [tw] OR prospectiv ${ }^{\star}$ [tw] OR volunteer ${ }^{\star}$ [tw]) NOT (animal [mh] NOT human [mh]) AND AND (Trichotillomania [mh] OR trichotillomania [tw] OR TTM [tw])

EMBASE (1980-2013) was searched using the following terms:

(random* OR "placebo":de OR control*) AND (trichotillomania:de)

PsycINFO (1970-2013) was searched using the following terms:

("randomisation" OR "randomisation" OR "controlled") AND ("trichotillomania")

\section{FEE D B A C K}

\section{First round editorial comments incorporated,}

\section{Summary}

First round of editorial comments received from Cochrane. Please see word document forwarded with full authors' responses to each point raised. Main issues were around consistency of reporting between abstract, plain language summary and summary of findings tables, and balancing reporting of positive and negative outcomes. 


\section{Reply}

Please see word document attached with full responses. All comments attended to and replied to.

\section{Contributors}

$\mathrm{JH}$ was main contributor. All co-authors provided input in this draft which incorporated the feedback.

\section{WHAT'S NEW}

\begin{tabular}{lll}
\hline Date & Event & Description \\
\hline 27 September 2021 & New search has been performed & $\begin{array}{l}\text { We updated the searches on 26 November 2020; four new studies } \\
\text { are included. }\end{array}$ \\
\hline 27 September 2021 & $\begin{array}{l}\text { New citation required and conclusions } \\
\text { have changed }\end{array}$ & \begin{tabular}{l} 
Review updated \\
\hline
\end{tabular} \\
\hline
\end{tabular}

\section{H I S T O R Y}

Protocol first published: Issue 1, 2009

Review first published: Issue 11, 2013

\section{CONTRIBUTIONS OF AUTHORS}

Rachel Rothbart and Taryn Williams conducted the initial review and wrote the first publication (Rothbart 2013). Jacob Hoffman and Taryn Williams conducted the update of the review. Dan Stein coordinated the review and assisted with writing. Jonathan Ipser contributed towards the methodology section of the protocol and provided feedback on the initial review. Naomi Fineberg and Sam Chamberlain provided feedback on subsequent modifications of the protocol, initial review and update to the initial review.

This update to the initial review was conducted by Jacob Hoffman and Taryn Williams and was coordinated by Dan Stein, who assisted with writing.

\section{DECLARATIONSOF INTEREST}

Jacob Hoffman: declares no conflicts of interest.

Taryn Williams: declares no conflicts of interest, outside of her employment by the Medical Research Council of South Africa.

Rachel Rothbart: declares no conflicts of interest.

Samuel Chamberlain: consults for Promentis, and leso Digital Health. He receives stipends from Elsevier for editorial work at Comprehensive Psychiatry; and at Neuroscience \& Biobehavioral Reviews. Samuel Chamberlain's role in this research was supported by a Wellcome Trust Clinical Fellowship (reference 110049/Z/15/Z).

Naomi Fineberg: declares that in the past three years, I have held research or networking grants from the European College of Neuropsychopharmacology (ECNP), UK National Institute for Health Research (NIHR), Horizon 2020, Medical Research Council (MRC) and the University of Hertfordshire. In the past three years, I have accepted travel or hospitality expenses, or both, from the British Association for Psychopharmacology (BAP), ECNP, Royal College of Psychiatrists (RCPsych), International College of Neuropsychopharmacology (CINP), International Forum of Mood and Anxiety Disorders, World Psychiatric Association, Indian Association for Biological Psychiatry, and Sun. In the past three years, I have received payment from Taylor and Francis and Elsevier for editorial duties. In the past three years, I have accepted paid speaking engagements sponsored by Abbott and Sun. Previously, I have accepted paid speaking engagements in various pharmaceutical industry-supported symposia and have recruited patients for various pharmaceutical industry-sponsored studies in the field of obsessive-compulsive disorder (OCD) treatment. I lead an NHS treatment service for OCD. I hold Board membership for various registered charities linked to OCD. I give expert advice on psychopharmacology to the UK Medicines and Healthcare products Regulatory Agency (MHRA) and the UK National Institute for Health and Care Excellence (NICE).

Jonathan Ipser: declares no conflicts of interest.

Dan Stein: has received research grants or honoraria, or both, from Lundbeck and Sun. 
The South Africa Medical Research Council (SAMRC) Anxiety and Stress Disorders Research Unit has received funding from most pharmaceutical companies involved with psychiatry in South Africa.

\section{SOURCES OF SUPPORT}

\section{Internal sources}

- MRC Unit on Anxiety Disorders, South Africa

\section{External sources}

- University of Cape Town, Cape Town, South Africa

\section{DIFFERENCES BETWEEN PROTOCOL AND REVIEW}

The differences identified below are between the protocol and this update of the original review (Rothbart 2013).

- JH and TW performed selection of studies and data extraction, not the original authors assigned to these tasks (JI and LH).

- We created online forms and data extraction sheets. JH used online forms for extraction, and TW used data extraction sheets for manual data extraction.

- We used Python for some data handling, particularly where data were available from ClinicalTrials.gov protocols in XML format.

- JH and TW assessed risk of bias, not the original authors assigned to these tasks ( $\mathrm{J}$ and LH). We did not use the CCDAN Quality Rating Scale, as the risk of bias tool has made it obsolete.

- One of the objectives stated in the protocol was to identify clinical and methodological factors that predict response to medication. We did not do this in this review update because of the small number of included studies and the lack of meta-analysis, but it remains possible in future updates of the review.

- Types of participants: we planned to tabulate age, gender and ethnicity to address the question of their possible impact on the effects of medication. However, we did not do this due to insufficient available data.

- We did not prepare a descriptor table as stated in the protocol. Instead, we refer readers to the Characteristics of included studies tables.

- Data collection and analysis section: we did not use the SRS 4.0 online systematic review system TrialStat for trial selection and data extraction purposes. Instead, we performed data extraction manually onto data extraction sheets (paper copies) and using a Google Form developed for this review.

- We did not make descriptive information for each trial freely available in the ESR Nexus electronic database, as we did not use the TrialStat program. Therefore, the stipulation of the TrialStat FRIENDS program that this be done no longer applied.

- Unit of analysis issues: we included cross-over trials that lacked a washout period if the drugs compared were of the same class and had similar properties (this was stated in the 'Unit of analysis issues' section).

- We added mood stabilisers (e.g. lithium), glutamate modulators (e.g. N-acetylcysteine) and cell signal transducers (e.g. inositol) as categories under 'Types of interventions' in the 'Methods' section.

- We added multi-arm analyses and multiple treatment meta-analyses as methodological options to maximise the use of all data.

- We included additional sensitivity analyses under 'Subgroup analysis and investigation of heterogeneity' in 'Methods': namely, exclusion of results based on last observation carried forward (LOCF) or observed cases (OC), exclusion of cross-over trials, exclusion of cluster-randomised trials and varying of intracluster correlation coefficients (ICCS) in cluster-randomised trials.

- We added GRADE profiling to the methodology, results and discussion sections, and included summary of findings tables to reflect the GRADE ratings.

- We added thresholds for magnitude of effect size based on those commonly used for Cohen's d (Cohen 1988).

- We changed the secondary outcome of 'Responsiveness to treatment of comorbid symptoms of depression' to 'Reduction of comorbid symptoms of depression' as 'responsiveness' implies a categorical, binary 'response', whereas a reduction on a continuous scale was the measurement employed.

\section{NOTES}

None. 